\title{
Novel Roles of Tobacco-associated Genes Underlying Disparate Survival in Appalachian Head and Neck Squamous Cell Carcinoma
}

\author{
Brenen William Papenberg \\ West Virginia University, bwpapenberg@mix.wvu.edu
}

Follow this and additional works at: https://researchrepository.wvu.edu/etd

Part of the Cancer Biology Commons

\section{Recommended Citation \\ Papenberg, Brenen William, "Novel Roles of Tobacco-associated Genes Underlying Disparate Survival in Appalachian Head and Neck Squamous Cell Carcinoma" (2020). Graduate Theses, Dissertations, and Problem Reports. 7981.}

https://researchrepository.wvu.edu/etd/7981

This Dissertation is protected by copyright and/or related rights. It has been brought to you by the The Research Repository @ WVU with permission from the rights-holder(s). You are free to use this Dissertation in any way that is permitted by the copyright and related rights legislation that applies to your use. For other uses you must obtain permission from the rights-holder(s) directly, unless additional rights are indicated by a Creative Commons license in the record and/ or on the work itself. This Dissertation has been accepted for inclusion in WVU Graduate Theses, Dissertations, and Problem Reports collection by an authorized administrator of The Research Repository @ WVU.

For more information, please contact researchrepository@mail.wvu.edu. 


\title{
Novel Roles of Tobacco-associated Genes Underlying Disparate Survival in Appalachian Head and Neck Squamous Cell Carcinoma
}

\author{
Brenen William Papenberg
}

Dissertation submitted to the School of Medicine at West Virginia University in partial fulfillment of the requirements for the degree of

\author{
Doctor of Philosophy in Cancer Cell Biology \\ Ivan Martinez, Ph.D., Chair \\ John Hollander, Ph.D. \\ Michael Ruppert, Ph.D. \\ Peter Stoilov, Ph.D. \\ Sijin Wen, Ph.D.
}

Scott Weed, Ph.D., Mentor

Cancer Cell Biology Program

Department of Biochemistry

Morgantown, West Virginia

2020

Keywords: HNSCC, Appalachia, 11q13, Cyclin D1, competing endogenous RNA

Copyright 2020 Brenen W. Papenberg 


\section{ABSTRACT}

\section{Novel Roles of Tobacco-associated Genes Underlying Disparate Survival in Appalachian Head and Neck Squamous Cell Carcinoma Brenen William Papenberg}

Head and neck squamous cell carcinoma (HNSCC) is an aggressive neoplasm primarily caused by tobacco consumption or human papillomavirus (HPV) infection. Incidence and mortality rates for HNSCC vary geographically. Appalachian residents consume tobacco products to a greater extent than national averages, a risk factor known to promote HPV-negative HNSCC through increased genomic instability. Male Appalachian patients display significantly worse relative survival than Appalachian females or non-Appalachian residents of either sex. Secondary analysis of available cancer registry outcome data from 2007-2013 indicates that white males with stage IV oral cavity/pharyngeal (OC/P) HNSCC are responsible for the decreased male survival observed within Appalachian regions. This disparity is seen in a subset of Appalachian states and is associated with lower relative survival in HPV-Associated subsites, suggesting a higher percentage of HPV-negative cancers occur in oral cavity regions typically associated with HPV infection. This increase is likely associated with higher smoking and smokeless tobacco usage in Appalachian states. An unbiased statistical analysis of gene copy number alterations (CNAs) from the Cancer Genome Atlas (TCGA)-HNSC national cohort indicates that smoking significantly correlates with amplification or deletions of genes found in several chromosomal cytobands. Of these, chromosome 11q13.3 and 11q13.4 (amplified) and chromosomes 9p21.3 and 9p24.1 (deleted) are the only altered segments that correlate with reduced overall survival in patients that smoked, independent of HPV status. 11q13 amplification is the most common CNA in HNSCC and enhances tumor progression and poor outcome due to protein overexpression. Transcriptome analysis of HNSCC with 11 q13 amplification indicates that 13 out of 17 amplified genes are overexpressed, including the oncogenic cell gene CCND1 (cyclin D1). Recently, 3'UTRs of select mRNAs have been shown to function as positive regulators of cancer progression by sequestering tumor-suppressive microRNAs (miRNAs) as competing endogenous (ce)RNA. Thus, a novel mechanism of poor outcome in 11 q13 amplified patients may be due to the 3'UTR regions of elevated 11 q13 transcripts serving to titrate tumor-suppressive miRNAs, permitting increased oncogenic progression. The overall hypothesis of this dissertation is that HNSCC patients in Appalachia have lower survival due to a larger number of individuals harboring tumors with 11 q13 amplification from tobacco- 
induced damage, resulting in increased transcript expression that downregulates tumor-suppressive miRNA functions. Predictive algorithms and secondary TCGA data analysis indicate that the CCND1 3'UTR contains multiple miRNA response elements expected to bind known tumor-suppressive miRNAs. Patient outcome data indicates that CCND1-amplified patients with 3'UTR containing transcripts have poorer overall survival than patients with CCND1 overexpression lacking the 3'UTR. Stratification by CCND1 expression reveals several altered protein targets including CCNB1, TYMS, and SERPINE1, that share miRNA response elements with CCND1 mRNA. Identification of tumor-promoting effects from $11 q 13$ transcripts provides alternative avenues for therapeutic intervention in this most aggressive form of HNSCC by identifying novel druggable transcription targets that can augment protein-based precision medicine strategies or by facilitating stratification of patients for clinical trials in patients with smoking-induced 11 q13 amplification in Appalachia or other regions of high tobacco use. 


\section{Dedication:}

I dedicate this dissertation to my partner and future wife Sarah. You are my light in the darkness and the wind in my sails. You have made me a better person than I could on my own and I cannot wait to spend the rest of my life with you. Thank you for your encouragement and confidence in my ability throughout my time as a graduate student. 


\section{Acknowledgements:}

It would have been nearly impossible to accomplish this without the support of my friends and family. When I was having doubts about my path in life, all of them pushed me to keep going and I realized that this was the right path for me.

Firstly, I want to thank my future wife Sarah for all of your love and support throughout my entire time in Morgantown. I truly appreciate you being patient with me even if spending this much time in Morgantown wasn't a part of your dream. Spending the last five and a half years with you have been the best of my life. I love the adventures that we have and the things that we have been able to see so far. You have been an inspiration to me, pushing me to become my best self in a way that I could not do myself. You are a that reason I wake up for every day, the reason I am happy to come home every day, and my best friend. I can't wait to spend the rest of my life with you.

To my family, I want to thank you for allowing me to explore my interests, even if it meant moving halfway across the country. To my parents Bill and Pam; this accomplishment would never have been possible without your love and support throughout my entire life. You instilled an environment that allowed me to explore my scientific interests and never wavered from allowing me to follow my dreams. You helped me grow into the person that I am today and I will be eternally grateful for that. To my brother Brydon, my sister in-law Erin, my nephew Kaleb, and my niece Kenley; thank you for being my home away from home and allowing me recharge with some family bonding and of course the constant supply of craft beer. Your love and support through the years have been a blessing. To the rest of my family, thank you for making it seem like no time has passed whenever I am rarely able to make it home for a holiday. Even though we spend so much time and distance apart, coming back to the Midwest feels like I have never left.

To my friends I met in Morgantown including Emily, Marshall, Adam, Ray and Kayla; thank you so much for being the support that I needed throughout our time in graduate school. You were always available to talk whenever I needed some advice on how to get through my day and always there to hang out to either celebrate success or to distract from my failures. We have a lot of good memories together that I will cherish forever, including our nights at the townhouse, the nights downtown, and our time in Canada. I know that the friendships we have developed will stand the test of time, even as we embark on our own adventures through life.

To my friends that I shared a laboratory with, including Steve and Jessica; thank you for all of the support through the years. You always helped when I needed someone to bounce ideas off of, when I needed help with an experiment, and when I just needed someone to talk to. You were both just always there. I will forever remember our trips to Keglers to get cheesy fries, no matter the occasion. Jessica, thank you for always knowing the perfect time to bring in a plate of cookies for the lab and for caring so much about others. Steve, thank you for turning me into a craft beer aficionado by continuing our Beer Friday tradition. To the rest of my lab, including undergraduates and residents; thank you for your contributions to this whether they be splitting my cells one time or helping me with the clinical side of HNSCC.

To my mentor Scott Weed; you have been an amazing mentor during this experience. You were willing to take me into your lab and follow the research wherever it took us, even to the point of requiring us to learn entirely new fields of science and establish our own place in them. I know that my work is a departure from your comfort zone, but I deeply appreciate you allowing me to explore mine. Your office was always open to us for us to discuss what was on our mind and you were always willing to listen and do something about it.

To my committee; thank you for your guidance throughout my time here. I know that I ventured into some corners of science that were new to you, but you stuck it out with me and helped as much as you could and ultimately made my projects better.

Finally, to the entire Cancer Cell Biology program at West Virginia University; thank you for listening to my seminars and posters and not being afraid to give me constructive criticism. Thank you for always having that one missing reagent that I need to finish an experiment. Your willingness to help any other student in need is one thing I have loved about attending here. 


\section{Table of Contents}

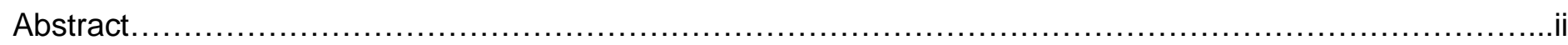

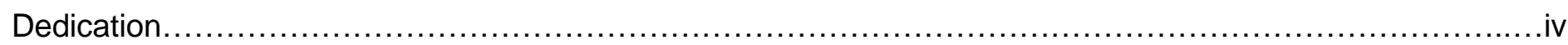

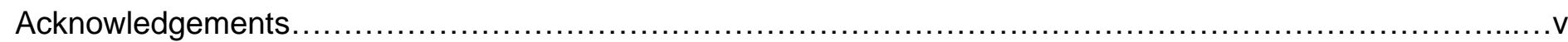

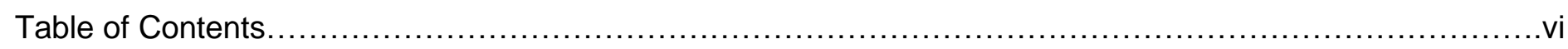

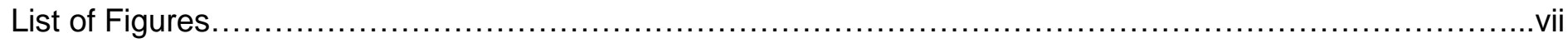

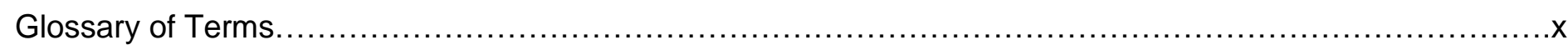

Literature Review

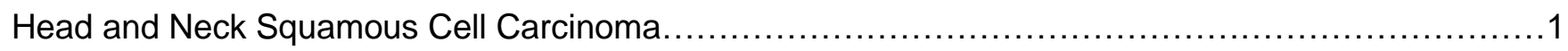

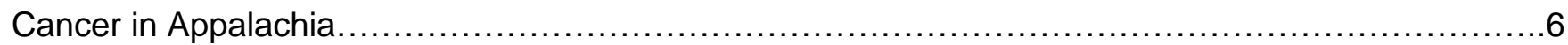

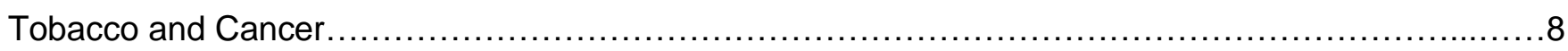

Chromosomal Instability and Amplification of Chromosome 11q13 ....................................11

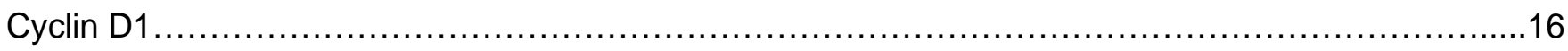

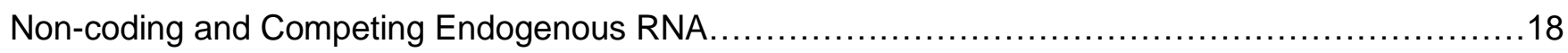

Study 1: Disparate Survival of Late-stage Male Oropharyngeal Cancer in Appalachia............................54

Study 2: Copy Number Alterations Identify a Smoking-Associated Expression Signature

Predictive of Poor Outcome in Head and Neck Squamous Cell Carcinoma................................87

Study 3: The CCND1 3'UTR as a Potential Driver of Poor Outcome in Chromosome

11q13-amplified Head and Neck Squamous Cell Carcinoma............................................130

Addendum to Study 3: The CCND1 3'UTR as a Potential Driver of Poor Outcome in Chromosome

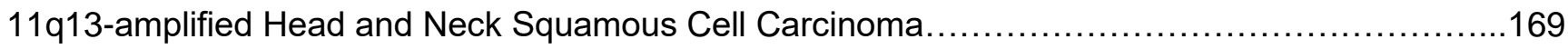

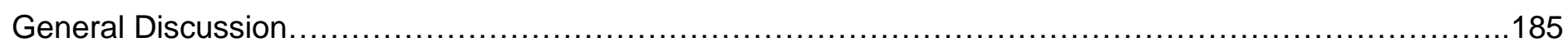

Appendix

Contribution: Cortactin Phosphorylation by Casein Kinase 2 Regulates Actin-Related

Protein 2/3 Complex Activity, Invadopodia Function, and Tumor Cell Invasion......

Commentary: When the Smoke Clears mA from a Y Chromosome-Linked IncRNA,

Men Get an Increased Risk of Cancer....................................................217

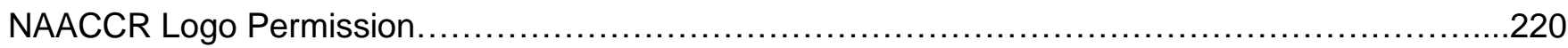

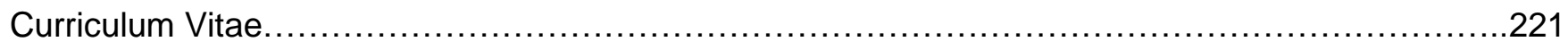




\section{List of Figures and Tables}

\section{Literature Review}

Figures

1. Characteristics of head and neck squamous cell carcinoma.

2. HNSCC patient relative survival by AJCC- 6 stage.

3. Cellular function of HPV viral proteins E6 and E7 and comparison of p16 staining between HPVpositive and HPV-negative patient derived xenografts (PDXs).

4. Incidence and Death rates of U.S. by state.

5. Characteristics of HNSCC supergroups.

6. Commonly altered pathways in HNSCC.

7. Smoking tobacco usage in United States, 1986-2010, 2011-2018.

8. Metabolism of NNN and NNK and formation of DNA adducts.

9. Link between cigarette smoke and cancer through carcinogens in tobacco smoke.

10. Mechanism of eukaryotic DNA replication under normal conditions and with a DNA adduct.

11. Diagrammatic representation of breakage-fusion-bridge mechanism.

12. Most commonly amplified 11 q13 genes from TCGA.

13. Transcriptional regulation of Cyclin D1 and regulation of cell cycle by Cyclin D1.

14. Biogenesis and function of cellular microRNA.

15. The ceRNA hypothesis at a glance.

\section{Study 1}

Figures

1. Identification of a Survival Disparity in White Appalachian Male Stage IV OC/P cancer.

2. State-level analysis of Appalachian OC/P survival.

3. The Stage IV White Appalachian Male OC/P survival disparity is predominant in HPV-associated oropharyngeal cancers.

4. High combined tobacco use in Stage IV white Appalachian male OC/P disparity states. Tables

1. Appalachian and Non-Appalachian OC/P and Laryngeal Patient Characteristics 2007-2013 Supplementary Figures

1. Survival analysis of Appalachian Stage IV laryngeal cancer.

2. Survival analysis of Appalachian states with non-significant OC/P survival.

3. Survival analysis of Appalachian states with non-significant OC/P survival.

\section{Study 2}

Figures 
1. 11 q13.3 and 11q13.4 amplification in HNSCC is correlated with genomic instability resultant from smoking.

2. $9 p 21.3$ and 9p24.1 deletion in HNSCC is correlated with patient smoking status.

3. 11q13 Amplification Patterns Identify a Distinct Chromosomal Segment Containing Smoking Overexpression Signature Genes in HNSCC.

4. Smoking-associated gene expression identifies a transcriptional signature that negatively impacts HNSCC outcome.

5. Predictive modeling and odds analysis of smoking-associated 11 q13 genes in HNSCC survival and lymph node metastasis.

Tables

1. Mutational Frequency of Common HNSCC-associated Genes in Smoking and non-Smoking Patients with 11 q13.3 and 11q13.4 Gene Amplification

Supplementary Figures

1. Determination of smoking-associated expression signature through effect of differential gene expression on overall survival.

2. Additive effect of SAES gene over- or underexpression on overall survival.

3. Relative risk of death of each smoking-associated expression signature gene.

Supplementary Tables

1. Clinical Characteristics of HNSCC Patients by Smoking, 11q13.3 and 11q13.4 Cytoband Amplification Status

2. Gene GISTIC2 Value and Pack-Year Linear Regression Analysis. Related to Figures 1 and 2. Available at WVU library.

3. Mutational Frequency of common HNSCC-associated Genes by Smoking Exposure. Related to Table 1.

4. Smoking-associated Gene Overexpression of HNSCC Patients by Smoking, 11q13.3 and 11q13.4 Cytoband Amplification Status. Related to Figure 4.

5. Multivariate Analysis Predicting Overall Survival using Age, Sex, AJCC Stage, HPV-Status, Extracapsular Spread, Perineural Invasion, and Lymphovascular Invasion.

6. Predictive Probability Linear Regression of Gene CNA by GISTIC2 Value and Pack-Year. Related to Figure 5.

7. Predictive Probability Linear Regression of Gene Over- or Underexpression by Z-score and PackYear. Related to Figure 5.

\section{Study 3}

Figures

1. The 11q13 amplicon contains genes with long 3'-UTRs that have tumor-suppressing MREs. 
2. The 3'-UTR of CCND1 is expressed in patients and is associated with poor survival.

3. Cyclin D1 protein and mRNA is overexpressed in amplified cell lines and mRNA localizes to cytosol.

4. Global mRNA expression is not altered by CCND1 3'-UTR overexpression.

5. Target protein regulation related to CCND1 3'-UTR expression in TCGA-HNSC.

6. Target protein regulation related to CCND1 3'-UTR expression in other 11q13-amplified cancers.

Supplementary Figures

1. Predicted MREs of genes within the $11 q 13$ amplicon.

\section{Addendum to Study 3}

Figures

1. Patient overall survival stratified by CCND1 exon expression.

2. Development of CCND1 constructs and expression in HNSCC cell lines.

3. Cell cycle analysis of CCND1 constructs in HNSCC cell lines.

\section{General Discussion}

Figures

1. Dissertation overview detailing three studies.

2. Possible outcomes of $\mathrm{p} 16 \mathrm{IHC}$ clinical test for prognosis. 


\section{Glossary of Terms}

9CFS - Common Fragile Site, Chromosome 9

ACS - American Cancer Society

AGO - Argonaute

AGO2 - Argonaute 2

AIAN - American Indian / Alaska Native (Race)

AJCC - American Joint Committee On Cancer

AKT - Protein Kinase B

ANO1 - Anoctamin 1, Calcium Activated Chloride Channel

ANOVA - Analysis of Variance

AP1 - Activator Protein 1

APA - Alternative Cleavage and Polyadenylation

API - Asian Or Pacific Islander (Race)

ARC - Appalachian Regional Commission

ARE - AU-Rich Element

ARE-BP - ARE-Binding Protein

AREG - Amphiregulin

Arp2/3 - Actin-Related Proteins 2 and 3

ATCC - American Type Culture Collection

ATM - Ataxia-Telangiectasia Mutated

B - Black (Race)

BA - Basal

BCL2L11 - Bcl-2-like Protein 11

BFB - Breakage-Fusion-Bridge

BID - BH3 Interacting Domain Death Agonist

BPDE - Benzo[2]Pyrene-7,8-Diol-9,10-Epoxide

BRAF - B-Raf

BRFSS - Behavioral Risk Factor Surveillance

System

C. elegans - Caenorhabditis elegans

CA9 - Carbonic Anhydrase 9

\section{Glossary of Terms}

CAK - CDK-Activating Kinase

CASP9 - Caspase 9

CCNB1 - Cyclin B1

CCND1 - Cyclin D1

CD8 - Cluster of Differentiation 8

CDC - Centers For Disease Control

$\mathrm{CDH} 3$ - Cadherin 3

CDK4 - Cyclin-Dependent Kinase 4

CDK6 - Cyclin-Dependent Kinase 6

CDKN2A - Cyclin-Dependent Kinase Inhibitor 2A

CDKN2B - Cyclin-Dependent Kinase Inhibitor 2B

CDSMD1 - CUB and Sushi Multiple Domains 1

ceRNA - Competing Endogenous RNA

CFS - Common Fragile Site

$\mathrm{Cl}$ - Confidence Intervals

circRNA - Circular RNA

CL - Classical

CMG - CDC45-MCM-GINS

CNA - Copy Number Alteration

CPT1A - Carnitine Palmitoyltransferase 1A (Liver)

CRS - Cumulative Relative Survival

CTTN - Cortactin

DCP2 - Decapping Protein 2

DEFB108B - Defensin, Beta 108B

DGCR8 - Digeorge Syndrome Critical Region 8

DHCR7 - 7-Dehydrocholesterol Reductase

DISC - Death-Inducing Signaling Complex

DMEM - Dulbecco's Modified Eagle Media

DNA - Deoxyribonucleic Acid

DVL3 - Dishevelled Segment Polarity Protein 3

E1-7 - Early 1-7 (HPV) 
E2F - E2 Transcription Factor

E6AP - E6AP ubiquitin-protein ligase

EGFR - Epidermal Growth Factor Receptor

ENPP7P8 - Ectonucleotide

Pyrophosphatase/Phosphodiesterase 7

Pseudogene 8

EPA - Environmental Protection Agency

ERAD - endoplasmic-reticulum-associated protein degradation response

ErbB - Erythroblastic Oncogene B

ERBB2 - receptor tyrosine-protein kinase erbB-2

ERK - Extracellular Signal-Related Kinase

EV - Empty Vector

FADD - Fas (Tnfrsf6)-Associated Via Death

Domain

FAM86C1 - Family With Sequence Similarity 86, Member C1

FDA - Food And Drug Administration

FDR - False Disovery Rate

FGF19 - Fibroblast Growth Factor 19

FGF3 - Fibroblast Growth Factor 3

FGF4 - Fibroblast Growth Factor 4

FGFR - Fibroblast Growth Factor Receptor

FGFR1 - Fibroblast Growth Factor Receptor 1

FGFR3 - Fibroblast Growth Factor Receptor 3

FRA11F - Fragile Site 11F

FRA11H - Fragile Site $11 \mathrm{H}$

FRA9C - Fragile Site 9C

FS - Frameshift

G1 - Growth 1 (Cell Cycle)

GAL - Galanin/GMAP Prepropeptide

GATA3 - GATA Binding Protein 3

GDAC - Broad Genomic Data Analysis Center

GEF - Guanine Nucleotide Exchange Factor
GISTIC - Genomic Identification Of Significant Targets In Cancer

GMAP - Galanin Message-Associated Protein

GPR - G-protein Regulator 1

GRB2 - Growth Factor Receptor-Bound Protein 2

gRNA - Guide RNA

GTP - Guanosine Triphosphate

HER2 - Human Epidermal Growth Factor Receptor 2

HIF1A - Hypoxia-Inducible Factor 1-alpha

HLA-DRA - Human Leukocyte Antigen DR Alpha

Chain

HNSCC - Head And Neck Squamous Cell

Carcinoma

HOXD - Homeobox D

HPV - Human Papillomavirus

HR - Hormone Receptor

IARC - International Agency For Research On Cancer

ICD-O - International Classification Of Diseases For Oncology

ICOS - Inducible T-cell Costimulator

IDeA - Institutional Development Awards

IGH - Immunoglobulin Heavy Chain Locus

IGHMBP2 - Immunoglobulin Mu Binding Protein 2

IHC - Immunohistochemistry

IMS - Inflamed / Mesenchymal

INBRE - IDeA Network of Biomedical Research Excellence

INK4 - Inhibitors Of CDK4

ITGA2 - Integrin Subunit Alpha 2

KMT2D - Histone-Lysine N-Methyltransferase 2D

KRT1 - Keratin, type II cytoskeletal 1

KRT9 - Keratin, Type I cytoskeletal 9

L1-2 - Late 1-2 (HPV) 
LAG3 - Lymphocute Activation Gene 3

LAR - Leukocyte Common Antigen-Related

LC-MS - Liquid Chromatography Mass

Spectrometry

let-7 - lethal-7

lin-14 - Abnormal Cell Lineage 14

lin-4 - Abnormal Cell Lineage 4

IncRNA - Long Non-Coding RNA

log2FC - log2 Fold Change

LOOCV - Leave-One-Out Cross Validation

LRP1B - Low-Density Lipoprotein Receptor-Related Protein 1B

MACC1 - Metastasis-associated in colon cancer protein 1

MAPK - Mitogen-Activated Protein Kinase

MAS - Mitochondrial Assembly

$\mathrm{Mb}$ - Megabases

MDM2 - Murine Double Minute

MEK - Mitogen-Activated Protein Kinase Kinase

MIR3164 - MicroRNA 3164

MIR3664 - MicroRNA 3664

MIR548K - MicroRNA 548K

miRISC - MicroRNA-Induced Silencing Complex

miRNA - MicroRNA 9

MM - miRNA-mutant Constructs

MMP9 - Matrix Metallopeptidase 9

MRE - MicroRNA Response Element

MRGPRD - MAS-Related GPR, Member D

MRGPRF - MAS-Related GPR, Member F

mRNA - Messenger RNA

MRPL21 - Mitochondrial Ribosomal Protein L21

MTL5 - Metallothionein-Like 5, Testis-Specific

(Tesmin)
mTOR - Mammalian Target Of Rapamycin

MYEOV - Myeloma Overexpressed

NAACCR - North American Association For Central Cancer Registries

NAD - Nicotinamide Adenine Dinucleotide

NADSYN1 - NAD Synthetase 1

NCBI - National Center for Biotechnology

Information

NCCN - National Comprehensive Cancer Network

$\mathrm{NCl}$ - National Cancer Insitute

NF1 - Neurofibromin 1

$\mathrm{NIH}$ - National Insitutes Of Health

NKX2-1 - NK2 Homoeobox 1

NNK - 4-(Methylnitrosamino)-1-(3-Pyridyl)-1-

Butanone

NNN - N-Nitrosonornicotine

NOTCH1 - Notch homolog 1

NRG1 - Neuregulin 1

NSD1 - Nuclear Receptor Binding SET Domain

Protein 1

OC/P - Oral Cavity And Pharyngeal

ORAOV1 - Oral Cancer Overexpressed 1

ORF - Open Reading Frame

PABC - Poly(A)-Binding Protein C

$\mathrm{PAH}$ - Polycyclic Aromatic Hydrocarbons

PCLO - Piccolo

PCNA - Proliferating Cell Nuclear Antigen

PCR - Polymerase Chain Reaction

PD-L1 - Programmed Death Ligand 1

PDX - Patient Derived Xenograft

PI - Propidium lodide

PI3K - Phosphoinositide 3-Kinase

PIK3CA - Phosphatidylinositol-4,5-bisphosphate 3Kinase Catalytic Subunit Alpha 
POB - Pyridyloxo-Butyl

PPFIA1 - Liprin, Alpha 1

PRAD1 - Parathyroid Adenomatosis

pRB - Retinoblastoma Protein

PRC2 - Polycomb Repressive Complex 2

PTEN - Phosphatase And Tensin Homolog

PTH - Parathyroid Hormone

PXN - Paxillin

QGIS - Quantum Geographic Information System

Raf - Rapidly Accelerated Fibrosarcoma

Ras - Rat Sarcoma

RING - Really Interesting New Gene

RNA - Ribonucleic Acid

RNA5SP342 - 5S Ribosomal Pseudogene 342

RNAse - RNA Sequencing

RNF121 - Ring Finger Protein 121

ROS - Reactive Oxygen Species

RPKM - Reads Per Kilobase of Transcript, Per Million Reads

RPPA - Reverse Phase Protein Lysate Microarray

rRNA - Ribosomal RNA

RS - Relative Survival

RTK - Receptor Tyrosine Kinase

S - Synthesis (Cell Cycle)

SAES - Smoking-Associated Expression Signature

SEER - Survival, Epidemiology, And End Reports

SERPINE1 - Plasminogen Activator Inhibitor 1

SES - Socioeconomic Status

SH3 - SRC Homology 3

SHANK2 - Sh3 And Multiple Ankyrin Repeat

Domains 2

Shh - Sonic Hedgehog

snoRNA - Small Nucleolar RNA
SOS1 - Son Of Sevenless Homolog 1

SOX2 - (Sex Determing Region Y)-box 2

SSE - Structure-Specific Endonucleases

stRNA - Small Temporal RNA

TCGA - The Cancer Genome Atlas

TCGA-BLCA - TCGA Bladder Urothelial Carcinoma

TCGA-BRCA - TCGA Breast Invasive Carcinoma

TCGA-ESCA - TCGA Esophageal Carcinoma

TCGA-HNSC - TCGA Head-Neck Squamous Cell Carcinoma

TCGA-LUSC - Lung Squamous Cell Carcinoma

TME CoBRE - Tumor Microenvironment Center of Biomedical Research Excellence

TMEM16A - Transmembrane Member 16A

TNFRSF6 - Tumor Necrosis Factor Receptor Superfamily Member 6

TNRC6A - Trinucleotide Repeat-Containing Gene $6 \mathrm{~A}$ Protein

TP53 - Tumor Protein 53

TP63 - Tumor Protein 63

TPCN2 - Two Pore Segment Channel 2

tRNA - Transfer RNA

TSB - Target Site Blocking

TSNA - Tobacco-Specific Nitrosamine

TTF-1 - Thyroid Transcription Factor 1

TYMS - Thymidylate Synthetase

UCSC - University Of California Santa Cruz

UNC93B6 - Unc-93 Homolog B6

UTR - Untranslated Region

VEGF - Vascular Endothelial Growth Factor

VEGFR - Vascular Endometrial Growth Factor Receptor

VIM - Vimentin

W - White (Race) 
WHO - World Health Organization

Wnt - Wingless/Integrated

WV CTSI - West Virtinia Clinical and Translational

Science Institute

Xist - X-inactie specific transcript

XPO5 - Exportin 5

ZNF705E - Zinc Finger Protein 705E 


\section{Literature Review:}

\section{Head and neck squamous cell carcinoma}

Head and neck squamous cell carcinoma (HNSCC) is an aggressive neoplasm that arises from squamous epithelial cells of the upper aerodigestive tract, encompassing the oral cavity, pharynx, and larynx(1). There are over 65,000 new HNSCC cases and 14,500 deaths estimated for 2020 , with a five-year relative survival rate in the United States of $65 \%$ for all races and American Joint Committee on Cancer (AJCC) stages(2). Risk factors for the development of HNSCC include alcohol and tobacco use(3) and high-risk human papillomavirus (HPV) infection(4) (Figure 1).

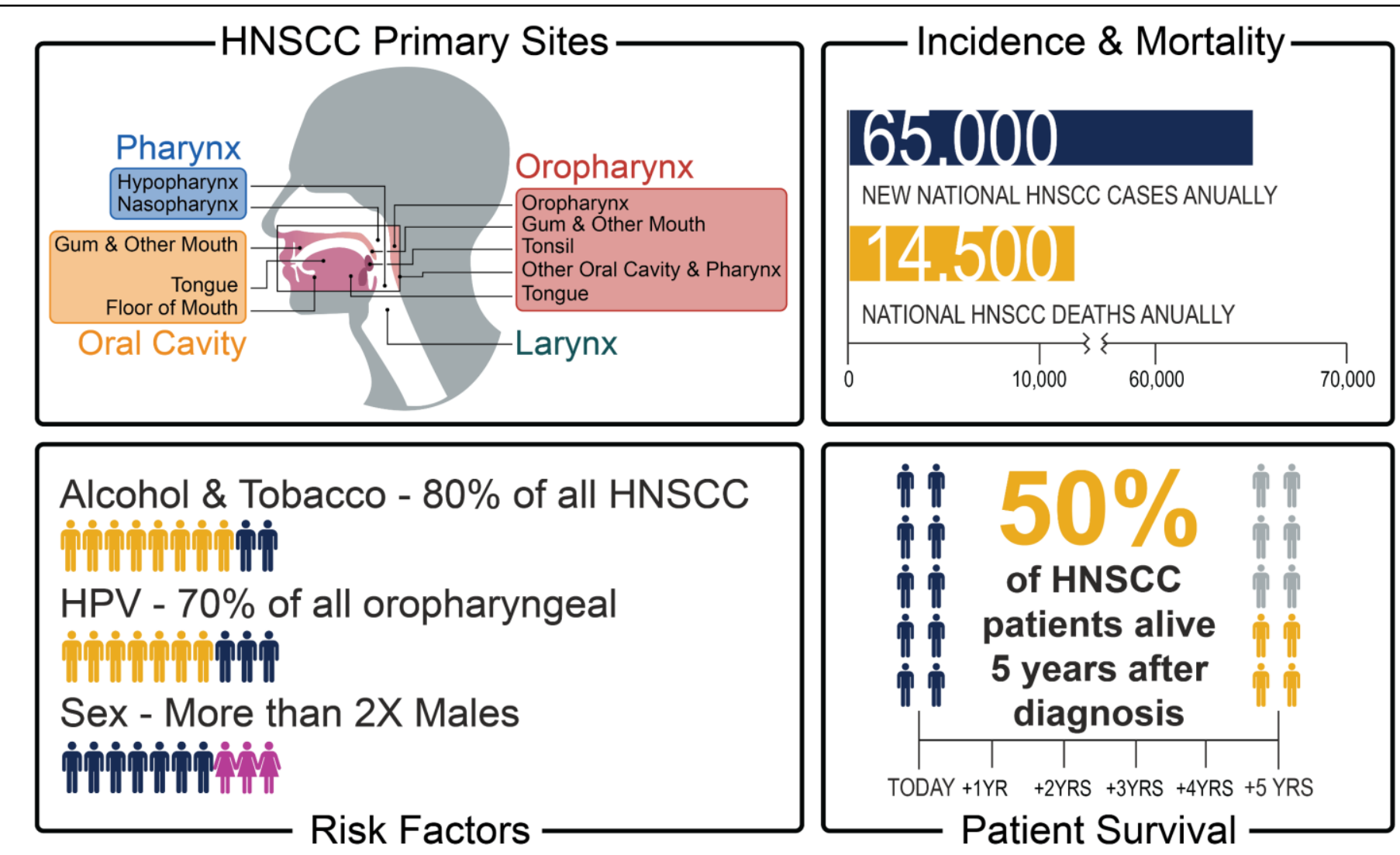

Figure 1. Characteristics of head and neck squamous cell carcinoma.

Treatment of HNSCC depends on anatomical site (surgical accessibility) and stage of disease. For early stage patients (AJCC stage I or II), which represent $30-40 \%$ of new diagnoses(1), HNSCC is curable with surgical resection or radiotherapy alone(5). For patients with locally advanced disease (AJCC stage III or IV), which represent over $60 \%$ of new diagnoses(1), surgical resection followed by adjuvant radiotherapy or 
chemoradiotherapy is used when surgical accessibility is feasible. Chemoradiotherapy alone is the curative standard of care when surgery is not feasible(5). Chemotherapeutic options for HNSCC include high dose cisplatin as the standard of care(5). To date, there are only two United States Food and Drug Administration (FDA) approved targeted therapies for HNSCC. The epidermal growth factor receptor (EGFR) inhibitor cetuximab was approved for use in 2006 to be used in certain circumstances, such as in cases with high EGFR where it was shown in clinical trials to increase 5-year survival when compared to radiotherapy alone(6), but platinum-based chemotherapy remains standard(5). However, if a patient tumor expresses the immune checkpoint protein programmed death ligand 1 (PD-L1), the patient is eligible for PD-L1 checkpoint inhibiting drugs such as pembrolizumab or
All Sexes, All Races, All Stages

ـ Derived AJCC-6 Stage I $\mathrm{N}=20,089$ | 5-year $=76.3[75.1-77.5]$ Derived AJCC-6 Stage II $N=14,020 \mid$ 5-year = $61.3[59.9-62.6]$

+ Derived AJCC-6 Stage III $\mathrm{N}=19,090 \mid$ 5-year $=56.8$ [55.5 - 58.0]

+ Derived AJCC-6 Stage IV $\mathrm{N}=63,396 \mid$ 5-year $=45.5[44.8-46.1]$

At 60 Months:

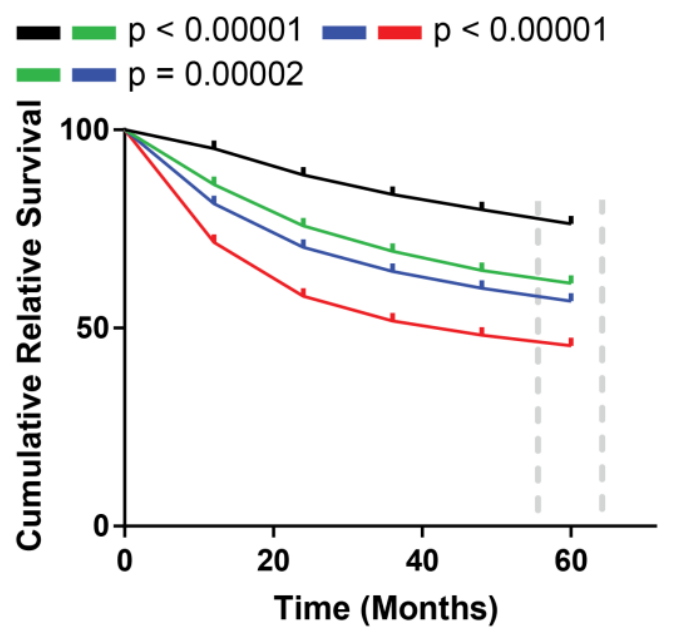

Figure 2. HNSCC patient relative survival by AJCC-6 stage.

nivolumab(5). Pembrolizumab has been shown in clinical trials to increase 5-year survival of recurrent or metastatic patients when compared to radiotherapy alone as well as cetuximab and radiotherapy(7). The lack of additional therapeutics for HNSCC treatment underscores the persistent need for the continued development of new drugs against known important gene products, as well as continued identification and testing of novel targets for improved treatments in populations more refractory to conventional clinical approaches. Patient survival is largely dependent on stage of diagnosis as well as treatment regimens (Figure 2), highlighting the need for additional treatment options.

Widespread next-generation sequencing of HNSCC cohorts has identified driver mutations, common tumorsuppressor and oncogenes, and molecular subtypes(8-11), concluding that HPV-positive tumors harbor fewer mutations and copy-number alterations (CNAs) than HPV-negative tumors. This is in agreement with HPVpositive patients exhibiting better overall survival and stratifies HNSCC into two distinct prognostic 
subtypes(12,13). HNSCC driver mutations identified in both HPV-positive and HPV-negative HNSCC are discussed throughout this review.

HPV-positive HNSCC incidence has been increasing and accounts for nearly $20 \%$ of all current HNSCC cases(14). HPV-positivity is significantly enriched in oropharyngeal tumors, where it is responsible for $60-80 \%$ of all HNSCC in this region(14-16). The remainder of HNSCC cases are HPV-negative and are described later in this review. High-risk HPV subtypes 16 and 18 are responsible for the majority of HPV-positive HNSCC, predominantly diagnosed in younger people after oral sex exposure(17) and is usually due to infection of the reticular epithelium lining the tonsillar crypts of the oropharynx(18-20).

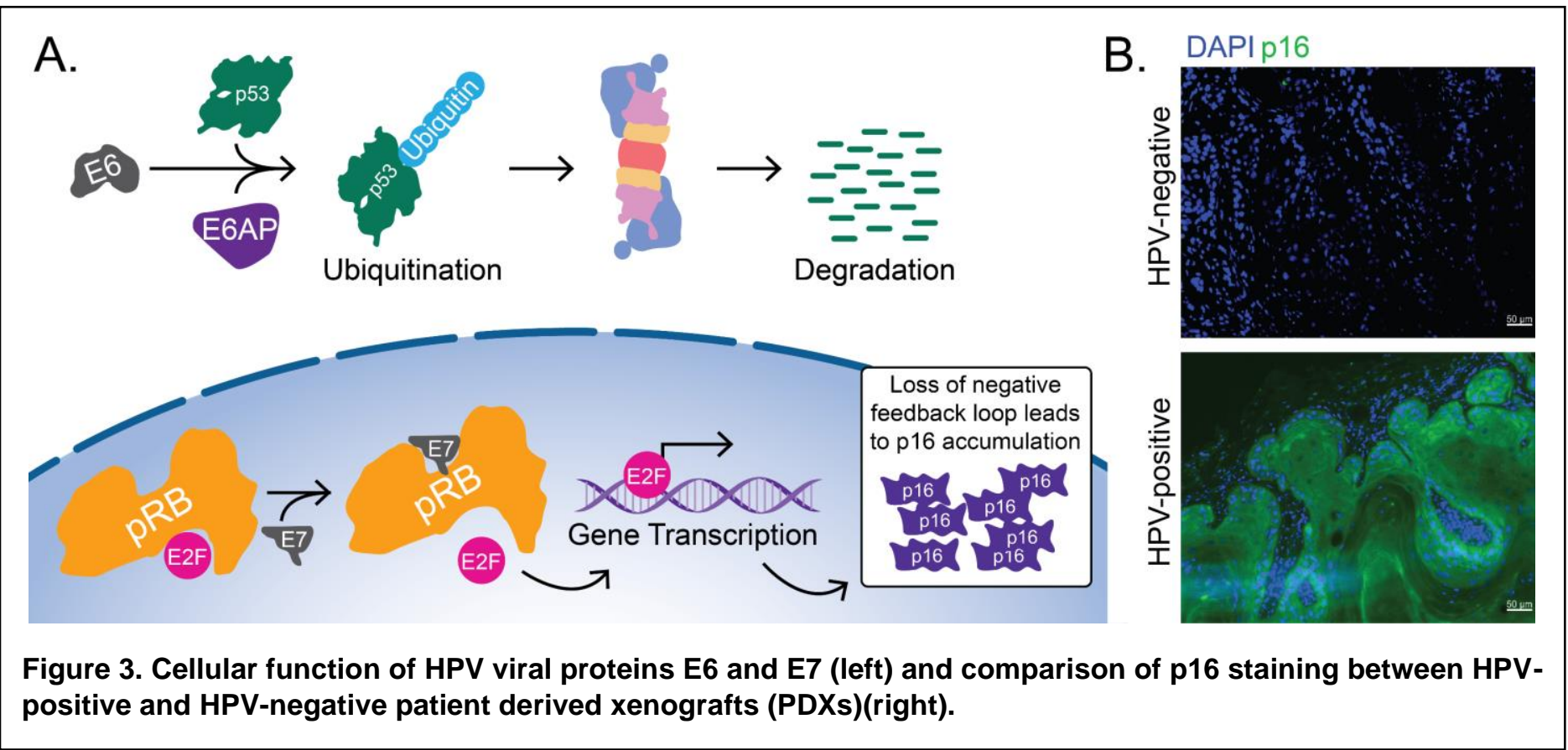

HPV has a double-stranded, circular genome with genes important for early viral functions (E1-7) that mostly act by blocking host cell division and viral genome replication, as well as genes involved in late viral functions (L12) such as capsid development(21). Early viral proteins E6 and E7 are oncoproteins that target the tumorsuppressors retinoblastoma (pRB) and p53, respectively (Figure 3A). E6 disrupts normal cell growth inhibition by inhibiting p53, leading to degradation of p53 through ubiquitination by ubiquitin-protein ligase E3A (E6AP)(22). E7 targets $\mathrm{pRb}$ family members for degradation, which results in sequential activation of the transcription factor E2F, expression of S-phase genes and ultimately hyperproliferation. Degradation of pRb and release of E2F also leads to overexpression of the tumor-suppressor p16, which is used as a surrogate marker for HPV-positive 
tumors as it is commonly overexpressed in HPV positivity tumors(23) (Figure 3B). As such, p16 immunohistochemistry $(\mathrm{IHC})$ is currently used by the National Comprehensive Cancer Network (NCCN) as a diagnostic step to determine treatment of oropharyngeal cancers(5).

While treatment for HPV-positive tumors has largely remained the same as treatment of HPV-negative tumors, the prospect of treatment de-escalation has been proposed due to the increased chemo-radiosensitivity and curative rate of HPV-positive tumors(24-26). To this end, AJCC revised staging of HPV-positive tumors in AJCC8, effective January 1, 2017, classifying most HPV-positive tumors as a lower stage(27). This is an important consideration for treatment guidance(5). However, recent studies have shown that some patients are undertreated using these guidelines, compromising patient safety(28). Prophylactic HPV vaccines have been approved for oropharyngeal cancer prevention, yet HPV-associated oropharyngeal incidence trends are not expected to reverse until after 2060(17). This expected delay is due to the vaccine being given to young individuals before sexual activity, the required time needed to achieve vaccine coverage in a population, and the time required to develop HPV-positive HNSCC(29).

HPV-negative HNSCC has a worse prognosis compared to HPV-positive HNSCC. This is mainly due to carcinogen-induced genomic instability that creates tumors with high mutational load, deleterious epigenetic alterations, large-scale chromosomal rearrangements and high intra-tumor heterogeneity (Figure 4). The aggregate effect of this genotoxic stress exerts a pleiotropic molecular impact on oncogenic processes that increases disease aggressiveness and lowers patient survival(8,9,30,31). The most common large-scale CNAs in HPV-negative HNSCC include amplifications of 7p11.2 (EGFR), 11q13.3 (CCND1), 8p24.21 (MYC), and 3p26.32 (PIK3CA) and homozygous deletion of 9p21.3 (CDKN2A). The most common somatic mutations in HPV-negative HNSCC include the tumor-suppressors CDKN2A and TP53. The most common CNA and somatic mutation in HPV-positive HNSCC is of PIK3CA(8).

To briefly describe these alterations, EGFR is part of the erythroblastic oncogene B (ErbB) family of receptor tyrosine kinases (RTKs) that responds to extracellular mitogens, driving downstream signaling via epidermal growth factor (EGF) binding, dimerization, autophosphorylation, and recruitment of adaptor proteins. EGFR has been shown to activate the Ras-Raf-MEK-ERK pathway described later in this review. The PI3K-AKT-mTOR 
pathway, which consists of phosphoinositide 3-kinases (PI3Ks) such as phosphatidylinositol-4,5-bisphosphate 3-kinase catalytic subunit alpha (PIK3CA), protein kinase $\mathrm{B}(\mathrm{AKT})$, and mammalian target of rapamycin (mTOR) regulates downstream molecules that are mainly related to metabolism and cell cycle(32). Activation of the RasRaf-MEK-ERK pathway can also induce Wnt (wingless/integrated) and Shh (Sonic hedgehog) signaling, which in turn can activate the transcription factor $M y c(33)$. Amplification of $E G F R$, amplification of $M Y C$, or mutation to PIK3CA lead to aberrant downstream signaling of these pathways and altered expression of various genes(34).

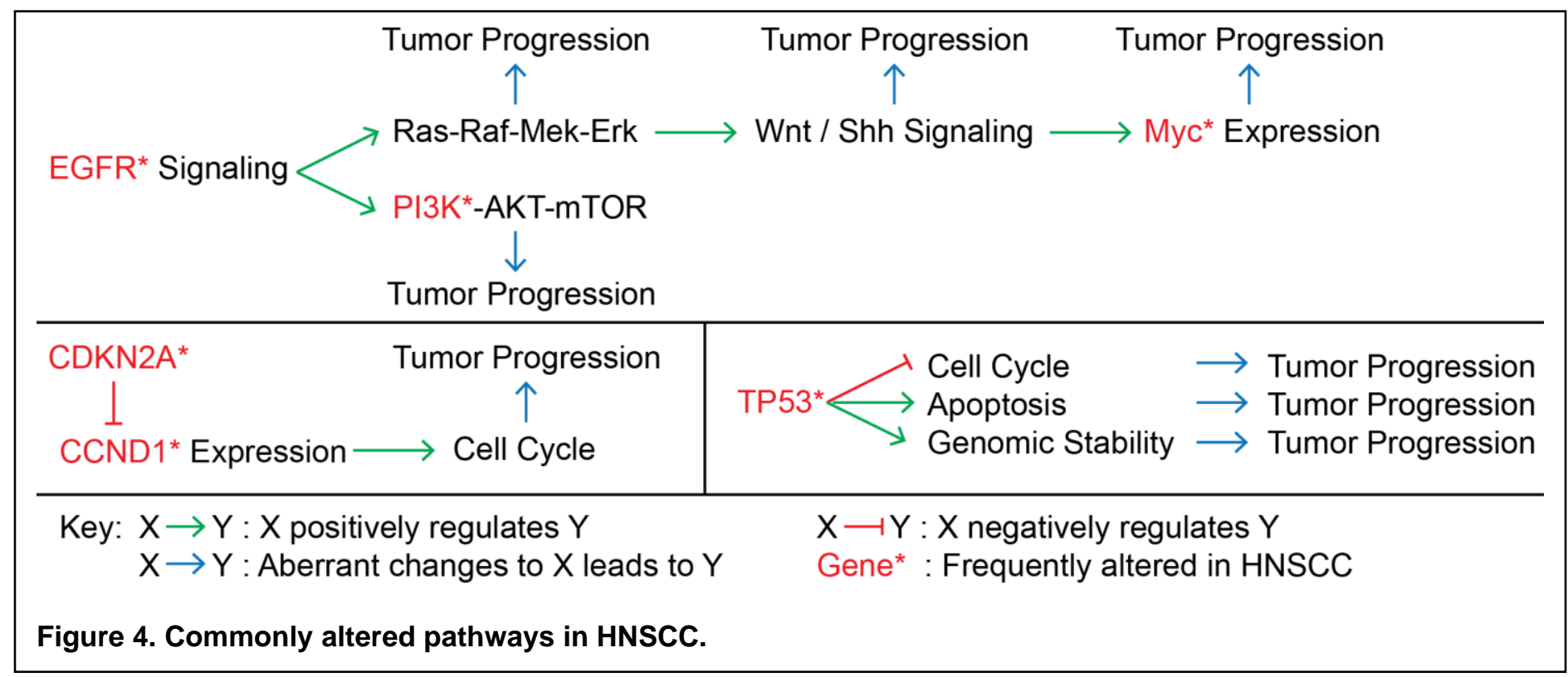

Cyclin D1 (CCND1) is a cell cycle regulator that is regulated by cyclin-dependent kinase inhibitor $2 \mathrm{~A}(C D K N 2 A)$.

These proteins are discussed in-depth later in this review. Tumor protein 53 (TP53) is a tumor-suppressing transcription factor that regulates the cell cycle, apoptosis, and genomic stability(35).

Combining genetic characteristics and gene expression profiles of HPV-negative HNSCC stratifies the disease into three distinct subtypes: basal (BA), classical (CL), and inflamed/mesenchymal subtypes (IMS)(9) (Figure 5). Characteristics of the BA subtype include enriched hypoxia signaling (HIF1A, CA9, and VEGF), neuregulin signaling (EGFR, AREG, and NRG1), and overexpression of the epithelial marker CDH3 and cytokeratins KRT1 and KRT9. This subtype is descriptive of nearly $40 \%$ of all cases and has been shown to be preferentially responsive to EGFR-target therapies(36). Characteristics of the CL subtype include enrichment for polyamine degradation pathways, which is related to tobacco detoxification. CL is also divided by HPV status into CL-HPV and CL-nonHPV, which make up $8.0 \%$ and $18.2 \%$ of all cases, respectively. While these two groups have similarities, they are still distinct in certain pathways, including cell-cycle and cell-division regulation. The IMS 
subtype features expression of genes related to T lymphocyte infiltration (CD8, ICOS, LAG3, and $H L A-D R A)$, overexpression of mesenchymal markers (VIM), overexpression of matrix metalloproteinases (MMP9), and downregulation of the epithelial marker $C D H 3$ and cytokeratins $K R T 1$ and KRT9. IMS is also divided by HPV status into IMS-HPV and IMS-nonHPV, two distinct subtypes that share characteristics, but differ in cell-cycle and smoking-associated pathways. They are responsible for $12.9 \%$ and $20.9 \%$ of all cases, respectively(9). Patient outcome differs between all subtypes, with the HPV-related subtypes CL-HPV and IMS-HPV having

\section{Basal Supergroup}

BA Subtype

$\uparrow$ Hypoxia Signaling (HIF1A, CA9, VEGF)

Neuregulin Signaling

(EGFR, AREG, NRG1)

$\uparrow$ Epithelial Markers

(CDH3, KRT1, KRT9)

\section{Classical Supergroup}

$\uparrow$ Polyamine Degradation

$\uparrow$ Cell Proliferation

$\uparrow$ Mini-chromosome Maintenance (MCM2, MCM10)

$\uparrow$ Cell Division Cycle Protein Kinase (CDC7, CDKN2A, E2F2, RPA2)

CL-nonHPV Subtype

$\uparrow$ Xenobiotic Metabolism

(CAKR1C1, AKR1C3, ALDH3A1)

\section{Inflamed / Mesenchymal Supergroup}

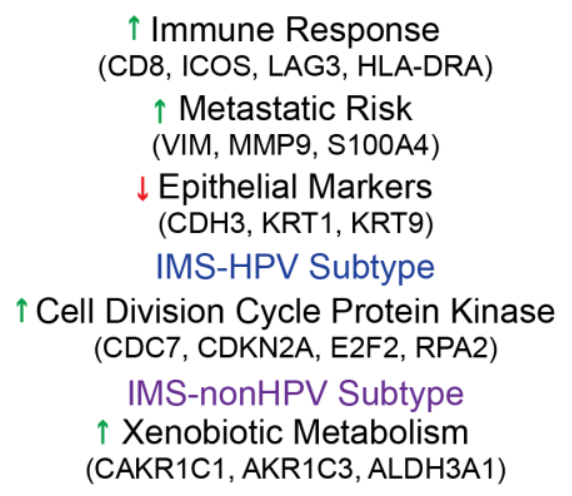

$\uparrow$ Immune Response

$\uparrow$ Metastatic Risk

(VIM, MMP9, S100A4)

Epithelial Markers

CDH3, KRT1, KRT9)

IMS-HPV Subtype

(CDC7, CDKN2A, E2F2, RPA2)

IMS-nonHPV Subtype

(CAKR1C1, AKR1C3, ALDH3A1)

Figure 5. Characteristics of HNSCC supergroups.

better overall survival than tobacco-related subtypes BA, CL-nonHPV, and IMS-nonHPV(9). Although many driving mutations in HNSCC have been extensively studied, most are related to alteration of protein expression. In Study 3, we evaluated the potential contributions to tumor progression through alteration of mRNA expression.

\section{Cancer in Appalachia}

Currently available data from the CDC show that cancer incidence and mortality rates have varied geographically (Figure 6) from 2013-2017 with higher rates present in the Appalachian region. The United States Appalachian region has a long history of diverse factors, such as low socioeconomic status (SES) and increased tobacco usage that contribute to the overall allostatic load responsible for increased cancer incidence and poorer patient outcomes(37). High cancer incidence in Appalachia has been recognized by the Appalachian Regional Commission (ARC) and the National Cancer Institute (NCI) as a health disparity since 1993, with high incidence rates remaining high in the region since that time(37-41). 
A. Incidence Rates (\%) by State, All Cancers 2013-2017, All Races, Sexes, Ages

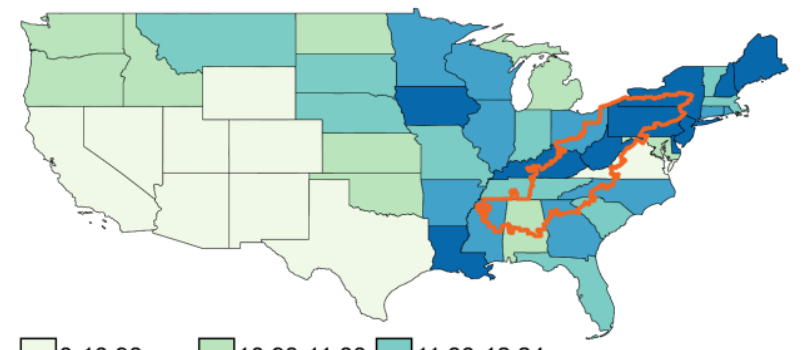

$\square$ 0-10.98 $\square$ 10.98-11.80 $\square$ 11.80-12.24
B. Death Rates (\%) by State, All Cancers 2013-2017, All Races, Sexes, Ages

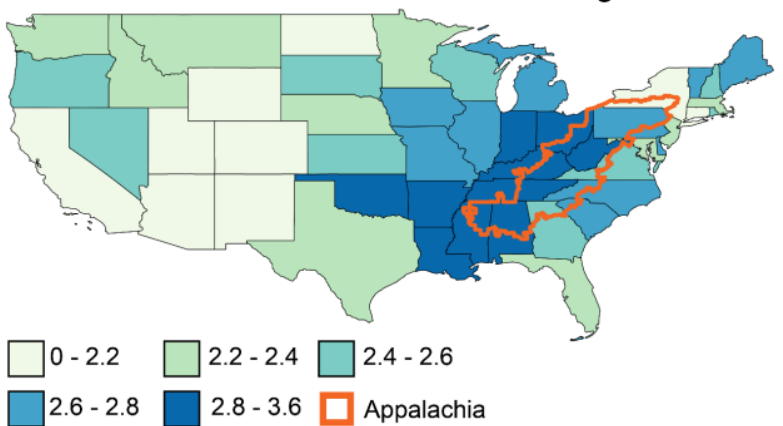

Figure 6. Incidence (A) and Death (B) rates of U.S. by state.

The United States Appalachian region currently encompasses 205,000 square miles across 420 counties in 13 contiguous states surrounding the Appalachian Mountains(37). The legal definition of Appalachia has changed multiple times since the ARC was first established in 1965 through amendments to United States Code(42). The demographics of the region portray a population that is over $80 \%$ white and $42 \%$ rural, with the region reporting higher unemployment, lower income, and higher poverty rates than most of the rest of the United States(43).

Recently, cancer incidence in general has been shown to have disparate and increasing incidence in Appalachia compared to non-Appalachia, regardless of sex, race, region, or primary site(38). However, this incidence disparity has decreased over time for most primary sites, with the exception of HNSCC (oral cavity, pharynx, and larynx) and cancers of the lung, bronchus, and thyroid(38), most of which are tobacco-associated malignancies $(38,39,44-47)$. Previous work evaluating oral cancer survival in Appalachia using 2004 Surveillance, Epidemiology, and End Results (SEER) data identified higher mortality in 10 Appalachian states including West Virginia, Alabama, Pennsylvania, Tennessee, Kentucky, Georgia, South Carolina, Mississippi,

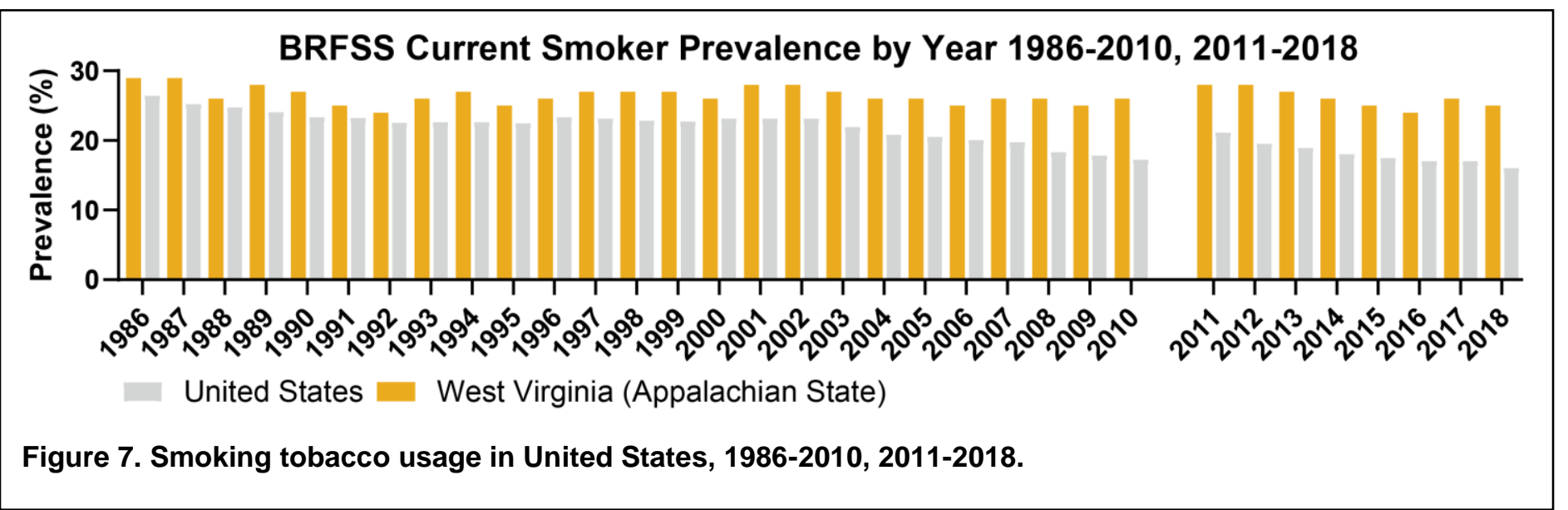


North Carolina, and Ohio(48). Of the cancer risk factors endemic to Appalachia, age at diagnosis, travel distance to critical care centers, and a disproportionate presentation of patients at a late AJCC stage have been reported to have no impact on survival in Appalachian subpopulations $(49,50)$. However, increased smoking and smokeless tobacco use for several decades within Appalachia have been linked to low SES and increased HNSCC incidence(51-53) and presumably contribute to HNSCC incidence and mortality disparities within Appalachia (Figure 7). A systematic evaluation within the Appalachian region to identify specific subregions or HNSCC sites responsible for driving disparate survival has not been conducted to date.

\section{Tobacco and Cancer}

There has been a presumed connection between tobacco usage and cancer development since Nazi Germany first investigated links between tobacco exposure and lung cancer in the late 1930s(54). The first clear evidence was published in 1954, which linked smoking habits to the mortality of British doctors(55). Evidence has mounted since then, and in 2004 the United States Surgeon General concluded that evidence is sufficient to infer a causal relationship between smoking tobacco usage and the development of tumors of the lung, larynx, oral cavity, pharynx, esophagus, pancreas, bladder, kidney, cervix, and stomach, as well as acute myeloid leukemia(56).

Incinerated tobacco products including cigarettes or cigars contain compounds that have been attributed to this relationship with cancer by previous work and the World Health Organization (WHO) International Agency for Research on Cancer (IARC). The most extensively investigated compounds include polycyclic aromatic hydrocarbons (PAHs), heavy metals, aldehydes, aromatic amines, and tobacco-specific nitrosamines (TSNAs). These compounds can be naturally produced by the tobacco plant or can be by-products from the combustion process, referred to as pyrolysis. Nicotine is a tobacco-generated alkaloid which the plant uses for defense against herbivorous insects(57) and is the addictive, stimulating substance within tobacco products due to its similarity to the neurotransmitter acetylcholine(58). Although nicotine is the addictive substance that drives tobacco consumption and uptake of tobacco-associated compounds, it has not been shown to be carcinogenic(56). PAHs are formed by the incomplete combustion of biological material. The United States

Environmental Protection Agency (EPA) has identified 16 key PAHs, including benzo[a]pyrene, that cause or may cause various types of cancer(59,60), all of which are found in tobacco smoke(61). PAHs have been shown 
to likely be involved in the carcinogenesis of lung, laryngeal, oral cavity, and cervical cancers(62). Heavy metals, including the human carcinogen cadmium $(60,63)$, are absorbed from the soil into the tobacco leaves $(64)$ and are released into tobacco smoke when incinerated(65). Heavy metals are likely to be involved in the carcinogenesis of lung cancer(62). Volatile carbonyl compounds, including acetaldehyde, formaldehyde, acetone, and 2-butanone, are formed from heating amino acids and sugars in the tobacco leaf(66) and are classified as possibly carcinogenic to humans by the $\operatorname{IARC}(60,67)$. Aldehydes are likely to be involved in the carcinogenesis of lung and nasal cancers(62). Aromatic amines, including 2-naphthylamine, are found in unburnt tobacco and are also formed as pyrolysis products(68) and are known human bladder carcinogens $(60,69)$. TSNAs are another class of chemical compounds that have been implicated in cancer development and include N-Nitrosonornicotine (NNN) and 4-(methylnitrosamino)-1-(3-pyridyl)-1-butanone (NNK), which have been shown to be carcinogenic $(60,70)$. TSNAs do not occur naturally within the tobacco plant and are not only a product of tobacco combustion, but are products of aerobic bacteria during drying and curing of tobacco(71). TSNAs are likely to be involved in the carcinogenesis of lung, nasal, oral cavity, esophageal, liver, pancreatic, and cervical cancers(62).

Smokeless tobacco, which is a tobacco product intended to be consumed without combustion but instead by direct absorption through the oral mucosa in the form of chewing tobacco, snuff, or snus, has a shorter list of carcinogenic compounds due to the lack of combustion. Smokeless tobacco does contain PAHs (benzo[a]pyrene), heavy metals (cadmium), carbonylic compounds (formaldehyde), and TSNAs (NNK and NNN), among others(72). Although smokeless tobacco still contains similar carcinogenic compounds when compared to smoking tobacco, the levels of these compounds are lower in smokeless tobacco(73). Electronic cigarettes are an interesting area in the development of a safer form of tobacco consumption. Several studies have shown that electronic cigarettes deliver a lower concentration of TSNAs $(74,75)$, but more research is required to determine how safe electronic cigarettes are in general or as a replacement for traditional smoking or smokeless tobacco(76). 


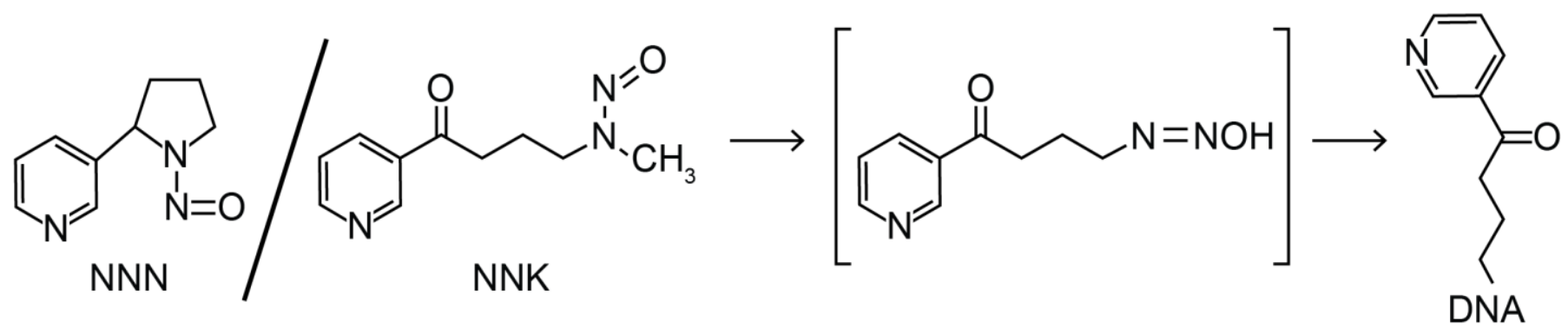

Figure 8. Metabolism of NNN and NNK and formation of DNA adducts.

The carcinogenic compounds within tobacco typically require metabolic activation, usually catalyzed by cytochrome P-450 enzymes, to convert them into a form that can covalently bind to DNA and form a DNA adduct (Figure 8). Differences in the efficiency of this metabolic activation or carcinogen detoxification may be able to explain cancer susceptibility between individuals(77). Two classes of tobacco-associated carcinogens that have been extensively studied concerning DNA adducts include PAHs and TSNAs. The molecular activation of benzo[a]pyrene (PAH) occurs through conversion to benzo[2]pyrene-7,8-diol-9,10-epoxides (BPDEs). This molecule forms adducts with deoxyguanosine to form BPDE-N2-deoxyguanosine(78). The molecular activation of NNN through $\alpha$-hydroxylation creates the same intermediate as methyl hydroxylation of NNK, pyridyloxo-butyl (POB)-DNA adducts(79). There are several mechanisms that cells can use to remove tobacco-generated DNA adducts, such as alkyltransferases, nucleotide excision repair, mismatch repair, and double strand break repair $(62,80)$. However, if the enzymes required for these repair mechanisms are unable to repair the DNA adduct, either through loss-of-function via mutation or being utilized elsewhere to repair widespread DNA damage, these DNA adducts can persist and be converted to genomic mutations through error-prone repair mechanisms or incorrect nucleotide insertion during translesion DNA synthesis(80) (Figure 9). Double stranded DNA breaks can also occur through oxidative damage to DNA linked to tobacco exposure(81). These double strand breaks can cause large chromosomal aberrations which can result in deletions and amplifications of genomic DNA(82). The tumor-suppressor p53 is one of the most important proteins for detecting and repairing DNA damage(83). Thus it is not surprising that mutations to TP53 are the most common mutations in human malignancies(84), preventing the cell to properly address DNA adducts, resulting in the eventual accumulation of mutations and chromosomal aberrations $(85,86)$. 


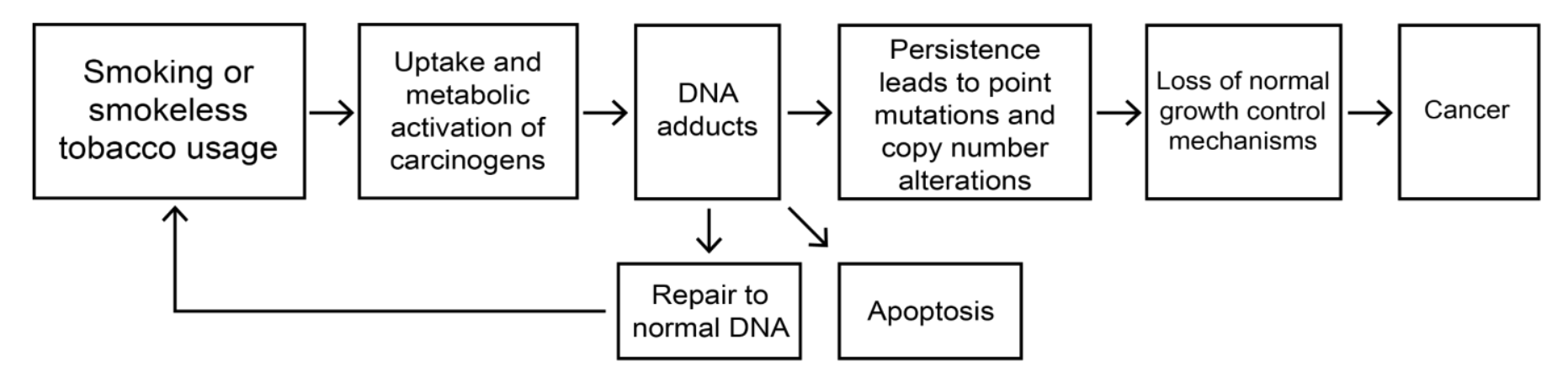

Figure 9. Link between cigarette smoke and cancer through carcinogens in tobacco smoke. Adapted from U.S. Department of Health and Human Services. How Tobacco Smoke Causes Disease: The Biology and Behavioral Basis for Smoking-Attributable Disease, p.226.

\section{Chromosomal Instability and Amplification of Chromosome $11 q 13$}

Chromosomal instability is a hallmark of cancer(87) that is defined as the rate of genetic aberrations seen within a cell or tumor and includes small mutations, insertions, and deletions. Instability also includes larger chromosomal rearrangements such as translocations and amplifications, and also includes generation of aneuploidy through gain or loss of entire chromosomes(88). Chromosomal instability is categorized into two main groups. The first is numerical chromosomal instability, which depicts the gain or loss of entire chromosomes. The second is structural chromosomal instability, which characterizes large chromosomal fragment changes that include chromosomal rearrangements and CNAs. CNAs include amplifications (more than two extra copies, +2 ), gene gains (two extra copies, +1 ), heterozygous deletions (missing one copy, -1 ), or homozygous deletions (missing both copies, -2$)(88,89)$. Chromosomal instability has been linked to cellular transformation, tumor heterogeneity, metastasis, tumoral drug resistance, and worse patient outcomes(90-93).

CNAs that arise from chromosomal instability are important areas of research due to the multitude of lost tumorsuppressors and gained oncogenic drivers which shape tumor progression. CNAs can range in size from focal alterations that include relatively few genes and comprise less than $25 \%$ of the length of a chromosome arm or alterations smaller than $3 \mathrm{Mb}$, to larger arm-length alterations that can encompass the length of an entire chromosome arm $(94,95)$. The shorter length of focal CNAs allows more straightforward determination of driving alterations, which remains difficult in large scale, arm-length alterations. Pan-cancer analyses have shown that many CNAs are specific to cancer type, but there are common deletions and amplifications found shared 
amongst multiple cancers. The most common focal amplifications across multiple cancer types include MYC, CCND1, ERBB2, CDK4, NKX2-1, MDM2, EGFR, FGFR1, and KRAS- all of which are documented oncogenes $(96,97)$. The most common focal deletions across multiple cancer types include those of $C D K N 2 A$, PTEN, and NF1 which are all tumor-suppressors $(96,97)$.

To briefly describe these alterations, receptor tyrosine-protein kinase erbB-2 (ERBB2/HER2), fibroblast growth factor receptor 1 (FGFR1), and EGFR are all RTKs that function in signaling transduction to activate the (KRAS)Raf-MEK-ERK pathway(34) that is regulated by neurofibromin 1 (NF1)(98), Wnt/Shh signaling which activates $M Y C(33)$, and the PI3K-AKT-mTOR pathway that is regulated by phosphatase and tensin homolog (PTEN)(32). Thyroid transcription factor 1 (TTF-1) or NK2 homeobox 1 (NKX2-1) is a transcription factor important for lineage survival as well as enhancing EGFR-driven tumorigenesis(99). Murine double minute (MDM2) functions an oncogene by inhibiting p53-mediated transactivation(100,101). CCND1 is a cyclin-dependent kinase 4 $(C D K 4) / C D K 6$ regulator that is involved in cell cycle regulation and is regulated by $C D K N 2 A$ that will be discussed in-depth later in this review.

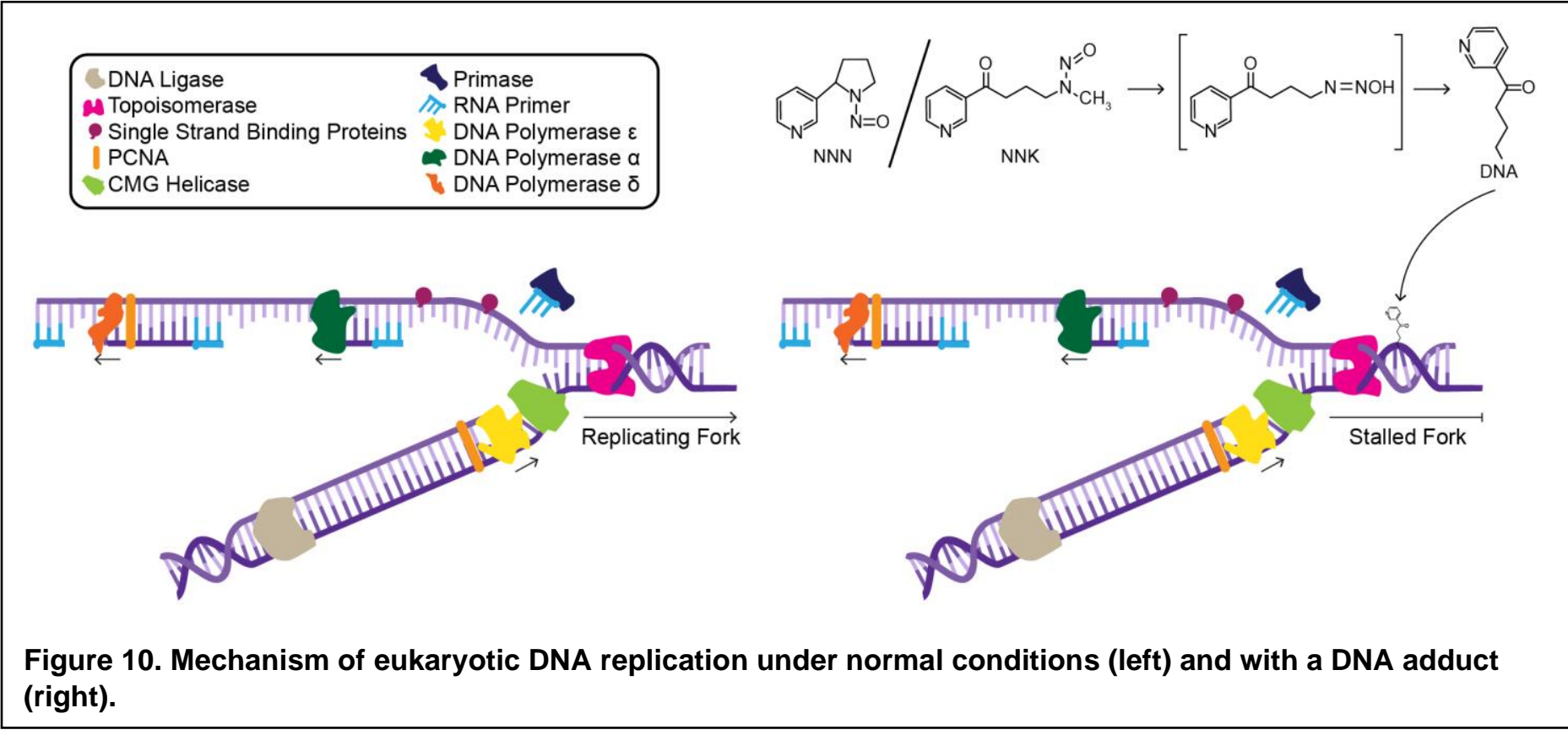

Fragile sites within normal chromosomes are sites of genomic instability during DNA replication stress. There are two classes of fragile sites, including rare fragile sites that are expressed (experience breakage) in less than $5 \%$ of individuals and common fragile sites (CFSs) that are found in all individuals(102-107). Fragile sites are 
usually located within large genes and have been linked to secondary structures of DNA and replication fork stalling due to replicative stress(107) (Figure 10). During replication, DNA strands are separated to expose the DNA template to DNA replication machinery, which allows for single stranded DNA to form secondary structures. If the replication fork is stalled due to replication stress, such as by tobacco-associated DNA-adducts as discussed in the previous section(108), more time is allowed for these secondary structures to form. This free, single strand DNA is then exposed to environmental damage and strand breaks may occur. Targeted cleavage of DNA by structure-specific endonucleases (SSEs) attempting to resolve the stalled fork can also lead to strand breaks(107). If stalled forks near fragile sites do not get resolved, DNA bridges may assist during nuclear division, which will lead to the mechanical breakage of DNA strands(107). Loss-of-heterozygosity or homozygous deletions can occur if multiple stalled forks are cleaved through deletion of the DNA between replication forks(107). If a double stranded DNA break remains unresolved during replication, it is possible for the broken strand to fuse to a sister chromatid and begin a breakage-fusion-bridge (BFB) cycle (Figure 11), which can lead to chromosomal amplification(109-111).

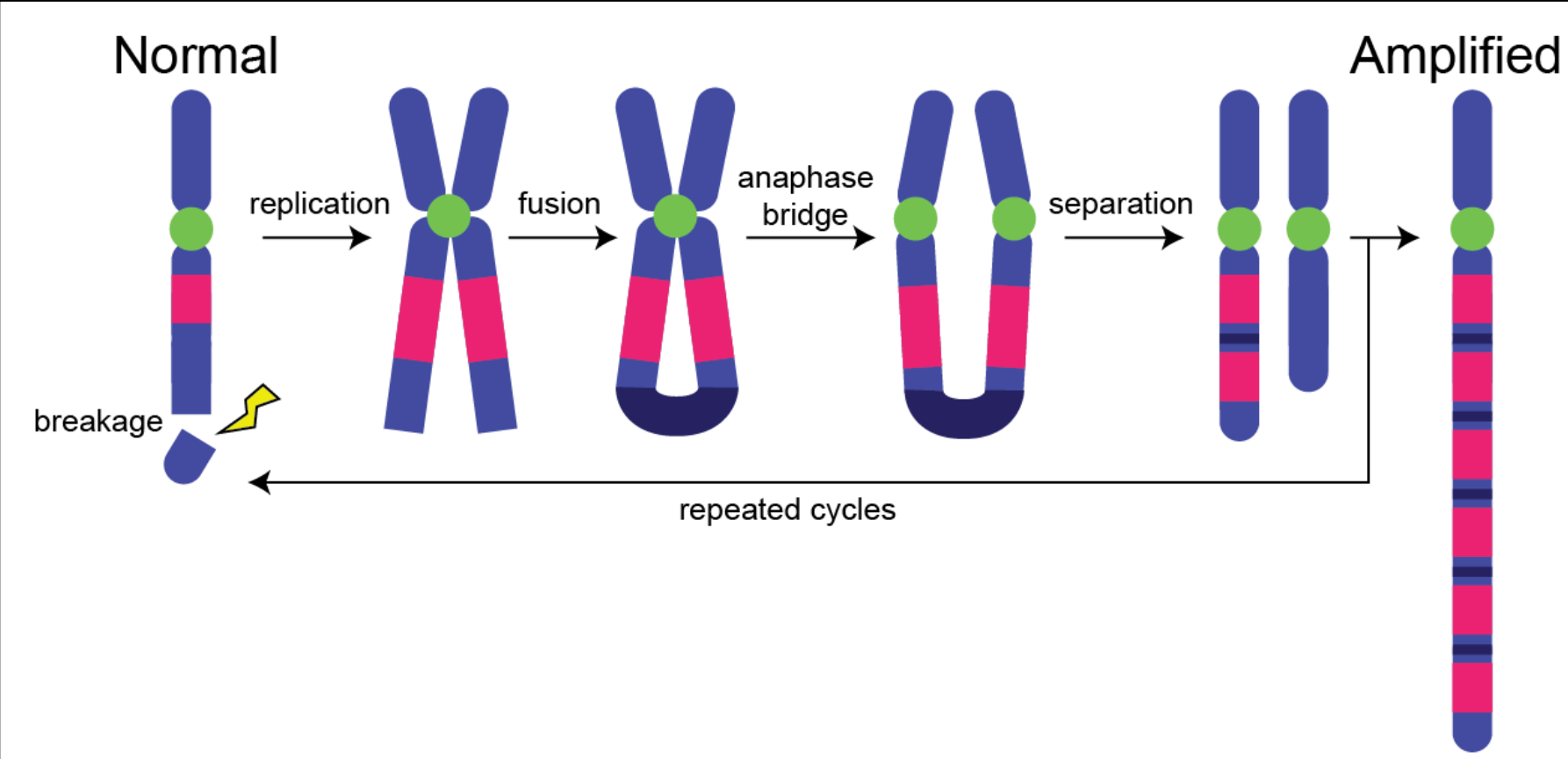

Figure 11. Diagrammatic representation of breakage-fusion-bridge mechanism.

When evaluating HNSCC for chromosomal aberrations, several alterations stand out and are common in an appreciable number of patients $(8,112,113)$. CNAs within HNSCC vary by HPV status $(8,112,114-117)$, with HPVnegative patients harboring a higher number of total CNAs, likely due to increased tobacco exposure $(8,112,113)$. 
The most common large-scale chromosomal amplification within HNSCC is the amplification of chromosome 11q13.3-q13.4. This amplification is found in nearly a third of all HPV-negative HNSCC patients and contains several genes of oncogenic interest, including the potential oncogenesis-promoting genes CCND1, FADD, and $\operatorname{CTTN}(8,113,118,119)$. The mechanism of $11 q 13$ amplification has been linked to $B F B(120)$ and appears to involve fragile sites $F R A 11 H$, and $F R A 11 F(121,122)$. However, other mechanisms such as amplification involving segmental duplications, which do not require the loss of a telomere, have also been proposed(123).

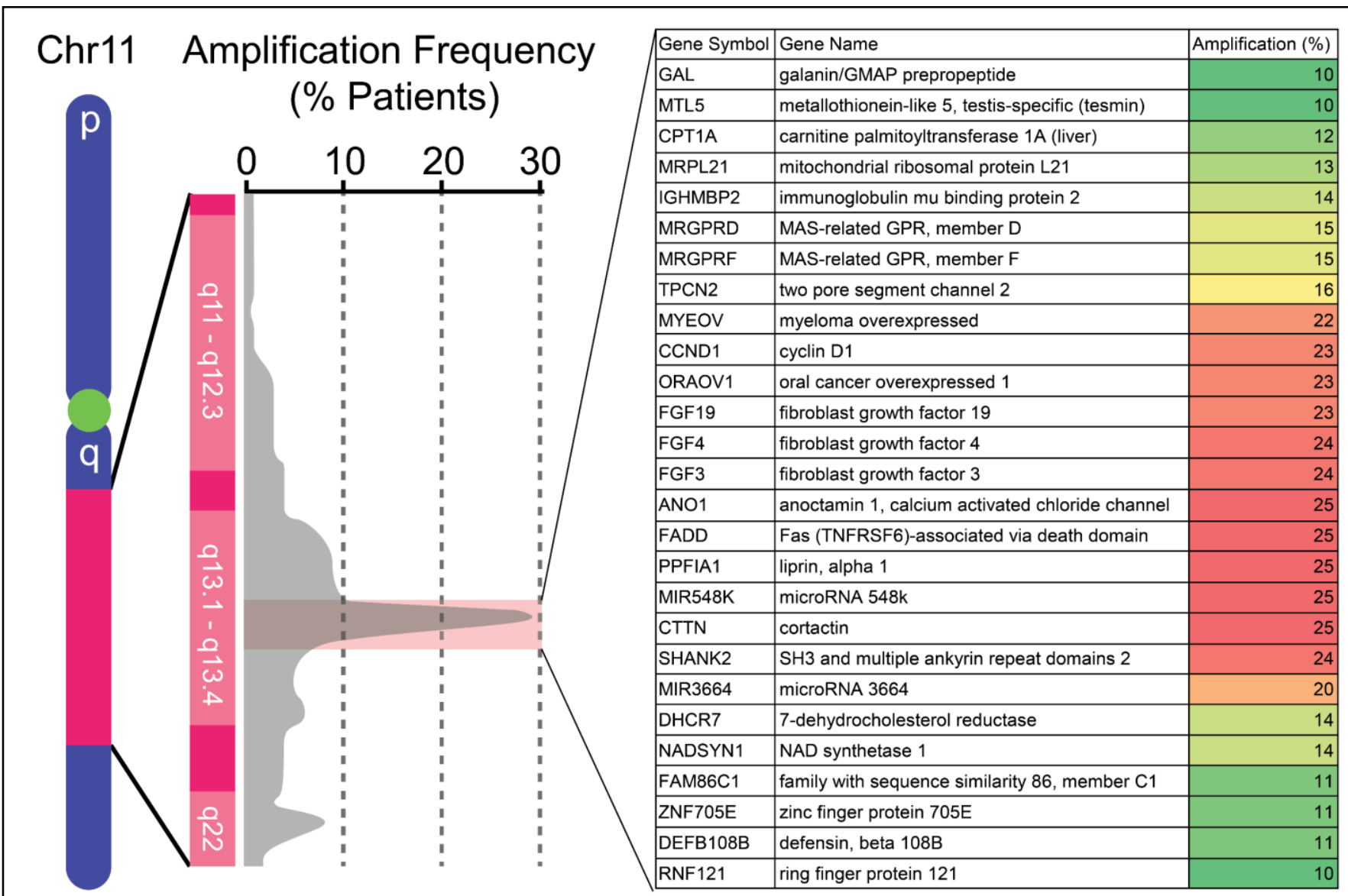

Figure 12. Most commonly amplified $11 q 13$ genes from TCGA.

Genes of note within the 11 q13 amplicon (Figure 12) include the cell cycle regulator CCND1 which will be discussed in depth later in this review. Briefly, cyclin D1 functions as the regulatory subunit of CDK4/6, whose activity is required for cell cycle transition from $\mathrm{G} 1$ to $\mathrm{S}(124)$. Overexpression of cyclin D1 protein leads to a constitutively active cell cycle and can lead to tumorigenesis(125). Oral cancer overexpressed 1 (ORAOV1/LTO1) has recently been shown to be involved in relief of reactive oxygen species (ROS)(126) and its overexpression has been shown to be a biomarker of progression(127). Liprin-a1 (PPFIA1) has been shown 
to localize LAR phosphatase to cell focal adhesions and has been shown to be involved in cell invasion, cell spreading, and cancer cell signaling(128-130). Anoctamin-1 (ANO1)/Transmembrane member 16A (TMEM16A) is a calcium-activated voltage-gated chloride channel that has been shown to play a role in inhibiting apoptosis(131). Fas-associated death domain $(F A D D)$ is involved in apoptosis by mediating the formation of death-inducing signaling complexes (DISC) during programmed cell death(132). Although FADD has been associated with HNSCC metastasis, its tumor-promoting functions are poorly understood(133,134). Fibroblast growth factor 3 (FGF3) acts as a negative regulator of bone growth through fibroblast growth factor receptor 3 (FGFR3)(135). Amplification and overexpression of FGF3 is associated with increased invasiveness in breast cancer(136). Fibroblast growth factor 4 (FGF4) functions in embryonic development (137) through an undetermined fibroblast growth factor receptor and is involved in human stomach cancer(138). Fibroblast growth factor 19 (FGF19) functions as a hormone in the regulation of bile acid synthesis and signals through fibroblast growth factor receptor 4 (FGFR4)(139). FGF19 amplification has been tied to autocrine signaling that promotes tumor growth(139). Cortactin (CTTM) is an actin-binding protein that regulates Arp2/3 and the branching of actin networks to facilitate cellular invasion, migration, and tumor metastasis(140-142). Myeloma overexpressed (MYEOV) appears to be involved in cell migration and is a prognostic marker in multiple myeloma and colon cancer(143,144). SH3 and multiple ankyrin repeat domains protein 2 (SHANK2) is a scaffold protein involved with actin dynamics at neuronal spines and synapses(145-147) and does not have an identified role in cancer. 


\section{Cyclin D1}

CCND1, originally referred to as parathyroid adenomatosis ( $P R A D 1)$, was first identified as a candidate oncogene in 1991 when it was found to be part of a genomic rearrangement with parathyroid hormone (PTH) in parathyroid adenomas, leading to its overexpression(148,149). Shortly after, it was implicated in genomic translocations in B-cell lymphoma where it was referred to as B-cell lymphoma 1 (BCL1)(150). PRAD1 was then confirmed to be a novel cyclin. Cyclins were identified due to their cyclic accumulation and subsequent destruction that varied depending on stage of cell cycle through the fertilization of marine invertebrate eggs(151). At the time of this discovery, two types of human cyclins had been characterized: A-type, important for the early cell cycle, and B-type, which drove the cell into mitosis. PRAD1 was similar in sequence to all cyclins, but could not fit into either group, suggesting it was part of a new cyclin family and was then renamed cyclin D1 (CCND1)(152,153). CCND1 was then confirmed to be a nuclear protein that is required during cell cycle progression in $\mathrm{G} 1(124)$.

The transcription regulation and cellular function of CCND1 has been elucidated in great detail (Figure 13, left). The best-studied activators of cyclin D1 transcription are mitogenic growth factors, which act through mitogenactivated protein kinases (MAPKs)

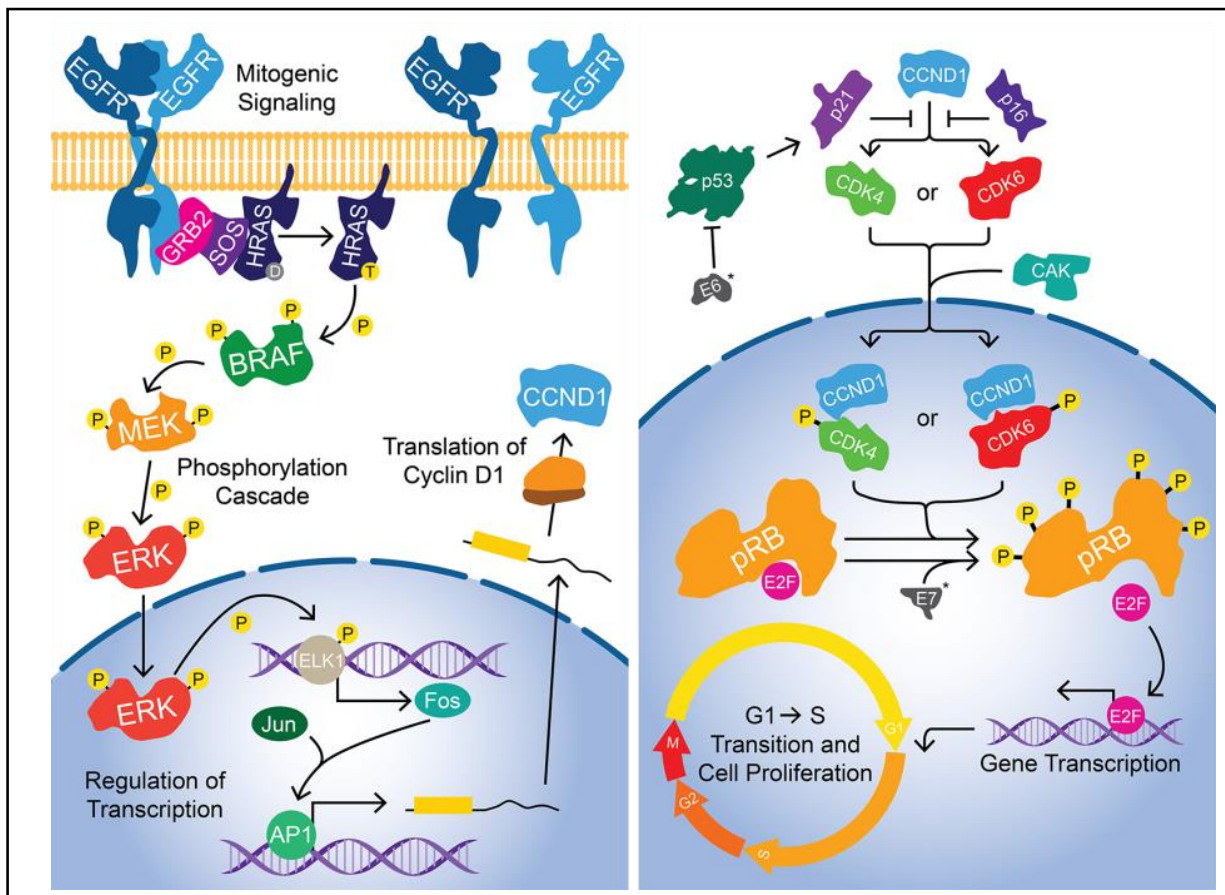

Figure 13. Transcriptional regulation of Cyclin D1 (left) and regulation of cell cycle by Cyclin D1 (right).

in the canonical Ras-Raf-MEK-ERK pathway(154). Briefly, small proteins that can induce a cell to begin mitosis through extracellular signaling are referred to as mitogens. These mitogens interact with RTKs on the cell surface such as EGFR, fibroblast growth factor receptor (FGFR), and vascular endometrial growth factor receptor (VEGFR)(155). After receptor-ligand binding, RTKs signal through adapter proteins and guanine nucleotide 
exchange factors (GEFs) such as growth factor receptor-bound protein 2 (GRB2) and son of sevenless homolog 1 (SOS1) which lead to the activation and release of the GTPase Ras (HRAS). HRAS will then begin a phosphorylation cascade through the Ras-Raf-MEK-ERK pathway which includes BRAF, mitogen-activated protein kinase kinase (MEK) and ultimately extracellular signal-related kinases (ERKs)(155). This pathway leads to the dimerization of Jun and Fos to produce the activator protein 1 (AP1) transcription factor and subsequent transcription of $C C N D 1(154)$. Once transcribed, $C C N D 1$ transcript can be regulated by both AU-rich elements (AREs) which stabilize the transcript and by microRNAs that can block translation or degrade the transcript(156). After translation, cyclin D1 protein functions as a regulatory partner with CDK4 or CDK6 (Figure 13, right). Cyclin D1 binds to CDK4/CDK6, allowing this complex to be activated by cdk-activating kinase (CAK)(157) where it enters the nucleus and then poly-phosphorylates pRB. This phosphorylation event causes a conformational change, allowing pRB to release pRB-bound E2F transcription factors which are responsible for the transcription of S phase genes(158,159). E2F target genes that are induced by this interaction include DNA polymerase alpha, thymidylate synthase, and ribonucleotide reductase, all of which are involved in DNA synthesis in S phase(160).

CCND1 has been established as a human oncogene via amplification or overexpression in breast cancer, lung cancer, melanoma, mantle cell lymphoma, and oral cancer(161). CCND1 overexpression predominately occurs because CCND1 is one of the most common CNAs in human cancer(96), but overexpression in mantle cell lymphoma is due to the translocation of CCND1 that places it next to the immunoglobulin heavy chain locus $(\mathrm{IGH})(162)$. The role of cyclin D1 in cancer is through increased CDK4/CDK6 activity, leading to increased pRB phosphorylation, hyperactivation of E2F-responsive genes and ultimately increased proliferation of tumor cells. However, there have been other non-canonical functions of cyclin D1 that have been suggested that can also contribute to its oncogenic ability. These functions fit into other hallmarks of cancer and include evading growth suppressors(163), activation of invasion and metastasis(164), enabling replicative immortality(165), inducing angiogenesis(166), and resisting cell death(167). The activity of CDK4/CDK6 is regulated by a family of tumorsuppressor proteins referred to as inhibitors of CDK4 (INK4). This family includes p16 INK4A, p15 INK4B, p18 INK4C, and p19 INK4D which inhibit CDK4/CDK6 by binding directly to the kinase $(168,169)$. Therapeutic targeting of the proliferate functions of cyclin D1 have been an area of interest which has focused on using small molecule selective inhibitors of CDK4/CDK6. To date, there are three FDA approved CDK4/6 inhibitors: 
palbociclib, abemaciclib, and ribociclib, all of which are approved for treatment of HR-positive and HER2negative breast cancer $(170,171)$. While clinical trials are still underway for other cancer types, HR-positive and HER2-negative breast cancer remains the only approved cancer for CDK4/6 inhibitor treatment(172).

\section{Non-coding and Competing Endogenous RNA}

A discrepancy between annotated RNA molecules and RNA molecules found within the human transcriptome has been noticed for some time(173-175). Recent studies have attempted to elucidate the function of some of these "junk" RNA molecules and have yielded new classes of RNA with new biological functions. To date there are many classes of RNA with varying functions including the canonical RNA involved in protein synthesis (messenger RNA, mRNA; ribosomal RNA, rRNA; transfer RNA, tRNA), RNA involved in DNA replication or posttranscriptional modification (small nucleolar RNA, snoRNA; guide RNA, gRNA) and RNA involved in regulatory functions (long non-coding RNA, IncRNA; microRNA, miRNA)(176), the focus of this review. Many of these RNA classes lack open reading frames (ORFs) and do not code for a protein product, which could solve the observed discrepancy.

One group of small regulatory RNA molecules are miRNAs, which are small RNAs that range from 20 to 30 nucleotides and do not have an ORF. The first miRNA was discovered in Caenorhabditis elegans in 1993 and was named small temporal RNA (stRNA) lin-4(177). It was then observed that lin-4 had complementary sequences to $3^{\prime}$ untranslated region (3'UTR) of the C. elegans gene lin-14 and was important for its temporal regulation during development(178). It took some time for other miRNA to be observed in $C$. elegans or in other species. The next miRNA to be observed was let-7 in C. elegans, which was again important for development. Excitingly, homologs for let-7 were found in humans and other animals(179). Multiple laboratories quickly found over 100 different small RNAs that came from a stem-loop precursor and were also evolutionarily conserved. However, these did not seem to be temporally expressed and were hence renamed to miRNA(180-182). To date, there have been thousands of miRNAs described in humans alone $(183,184)$.

Canonical biogenesis of miRNA begins with transcription by RNA polymerase II into pri-miRNAs(185) (Figure 14). These are then processed into pre-miRNAs by the microprocessor complex which includes RNA binding protein DiGeorge Syndrome Critical Region 8 (DGCR8) and the ribonuclease III enzyme Drosha(186). These 
pre-miRNAs are then exported into the cytoplasm by exportin 5 (XPO5) and are processed into a mature miRNA duplex by the RNAse III endonuclease Dicer, which cleaves the hairpin $\operatorname{loop}(186,187)$. These free strands of miRNA can then be loaded into the Argonaute (AGO) family of proteins(188) to form the miRNA-induced silencing complex (miRISC)(189) and miRNA-induced gene regulation can occur.

The miRISC complex can regulate gene expression via multiple mechanisms. The majority of miRISC gene regulation occurs through complementary sequences between the miRISC-bound miRNA and sequences in other RNA molecules called miRNA response elements (MREs). The

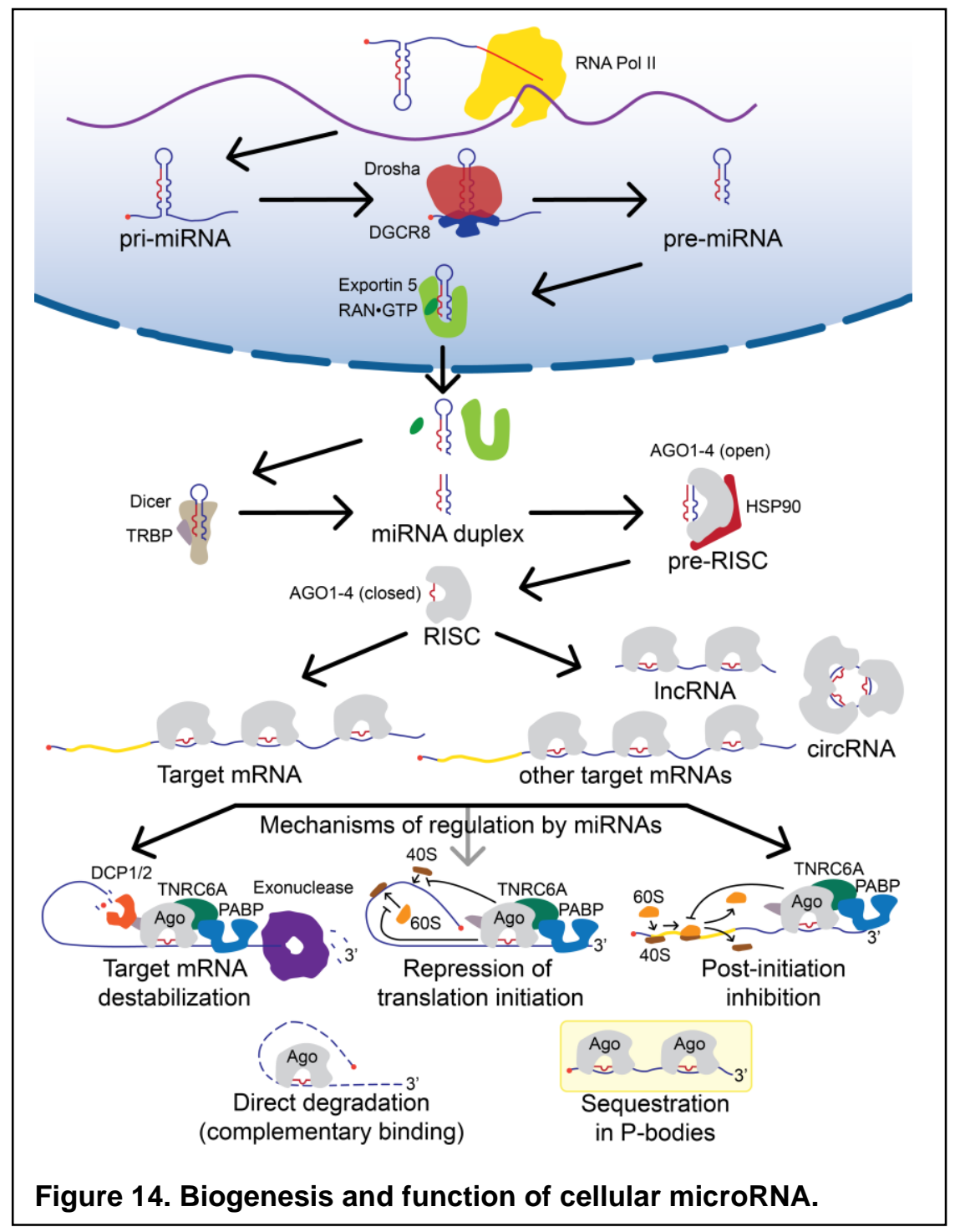
degree of complementary interaction between the miRNA:MRE and the AGO in the miRISC determines the mechanism of regulation. If the interaction is fully complementary and AGO2 is in the miRISC, this will result in direct AGO2-dependent endonuclease activity and degradation of the RNA(190). However, most miRNA:MRE interactions in animals are not fully complementary, so target degradation occurs through another mechanism. This begins with recruitment of trinucleotide repeat-containing gene 6A protein (TNRC6A/GW182) and poly(A)binding protein C (PABPC) which promotes deadenylation of the target transcript(191,192). Decapping of the transcript is then facilitated by decapping protein 2 (DCP2), which is then followed by target degradation by exonucleases(192,193). Not all miRISC interactions lead to immediate degradation of target RNAs through this mechanism. There is also evidence that miRISC can inhibit gene translation through blocking translation machinery initiating translation or inhibiting translation after it has initiated(190,194). It is also not necessary for 
this regulatory mechanism to result in degradation of target RNA, as multiple studies have shown that these interactions can result in a decrease in target protein without changes in target mRNA levels(195-198). Due to this observation, it is possible for miRNA to be sequestered or "sponged" by target mRNAs, IncRNAs, and circRNAs with shared MREs to mitigate their regulatory role(199).

Regulatory RNAs that are longer than miRNAs include long non-coding RNAs (IncRNAs). IncRNAs are RNA molecules that are longer than 200 nucleotides and either lack an ORF or code for small peptides less than 100 aa(200). IncRNA were first described in 1990 with the discovery of the regulatory IncRNA H19(201) and the Xinactivation linked IncRNA Xist(202). IncRNA can exist in the genome as stand-alone RNAs that are transcribed and polyadenylated(203), or as antisense transcripts opposite of sense coding genes(204), or as pseudogenes that have lost their coding potential due to mutations(200), or as intronic sequences(205), or as other promoterassociated and enhancing RNAs(206).

There have been many described IncRNAs involved in a variety of functions including epigenetic and transcriptional decoys and regulators. The IncRNA HOTAIR functions as a recruiter for the polycomb repressive complex 2 (PRC2) and regulates chromatin state of genes, including HOXD which is involved in differentiation and limb development(207). IncRNAs can also function as molecular recruiters by recruiting epigenetic complexes during transcription. The DNA:RNA complementarity of IncRNAs allow them to target these complexes to specific genomic positions, something that is not possible with protein-driven recruitment(208). Some IncRNAs act as decoy RNA and compete for transcription factor binding(209) while others can directly inhibit RNA polymerase II(210), both resulting in a downregulation in transcription. On the other hand, IncRNAs can also function as coregulators to activate transcription, such as ncRNA $A_{C \text { ND1 }}$ regulation during stress(154).

Aberrant expression levels of miRNAs and IncRNAs have been shown to be involved in cancer. In HNSCC, several oncogenic miRNA expression levels are altered, including miR-21 and miR-31, which are well documented oncogenic miRNAs that are detectable in plasma(211-215) and indicate a poor prognosis. miR-21 is the most frequently upregulated miRNA in HNSCC and is involved in drug resistance, invasion, cell survival, and metastasis(216). Well known targets of miR-21 include the tumor suppressors GRHL3 and PTEN(212,216219). miR-31 is also significantly upregulated in HNSCC and functions by targeting factor-inhibiting-factor (HIF), 
subsequently activating HIF and its downstream targets, ultimately contributing to the development of HNSCC $(220,221)$. Other aberrant miRNA expressions have been noted, especially in the case of gene amplification, but their cellular targets are still under investigation. Tumor suppressing miRNA can also experience expression changes in HNSCC, such as the let-7 family which includes let-7a-j and miR-98 that regulate cell renewal, cellular motility, and EMT regulation(222). The miR-99 family normally targets the mTOR signaling pathway to inhibit the phosphorylation of AKT. Downregulation of these miRNA, including through gene deletion, can lead to the initiation of HNSCC(223-225). Hundreds of IncRNAs have been shown to be differentially expressed in HNSCC(226) with the most extensively studied IncRNA being MALAT1, which is notably located on chromosome 11q13.1. High levels of MALAT1 are associated with increased lymph node metastasis and poor patient prognosis(227,228).

The presence of MREs on cellular mRNA, IncRNA, and other RNA molecules leads to the possibility that there is another layer of complexity involved in RNA regulation due to these molecules all competing for the same pool of cellular miRNA. This concept was first discussed in 2011 and is referred to as the competing endogenous RNA (ceRNA) hypothesis(229). Briefly, the hypothesis states that since the levels of MREs and miRNAs are in balance, any change to this balance, be it either from an increase in the amount of MREs through target gene overexpression or a decrease in miRNA due to mutation, will also alter expression of other target RNAs that share the same MREs(229-232) (Figure 15). This is still a controversial hypothesis as there is both ample evidence, such as ceRNAs that alter expression of the tumor suppressor PTEN(233) or BRAF(234) and stoichiometric concerns that state that changes in miRNA target abundance is unlikely to cause effects on gene expression(235). 


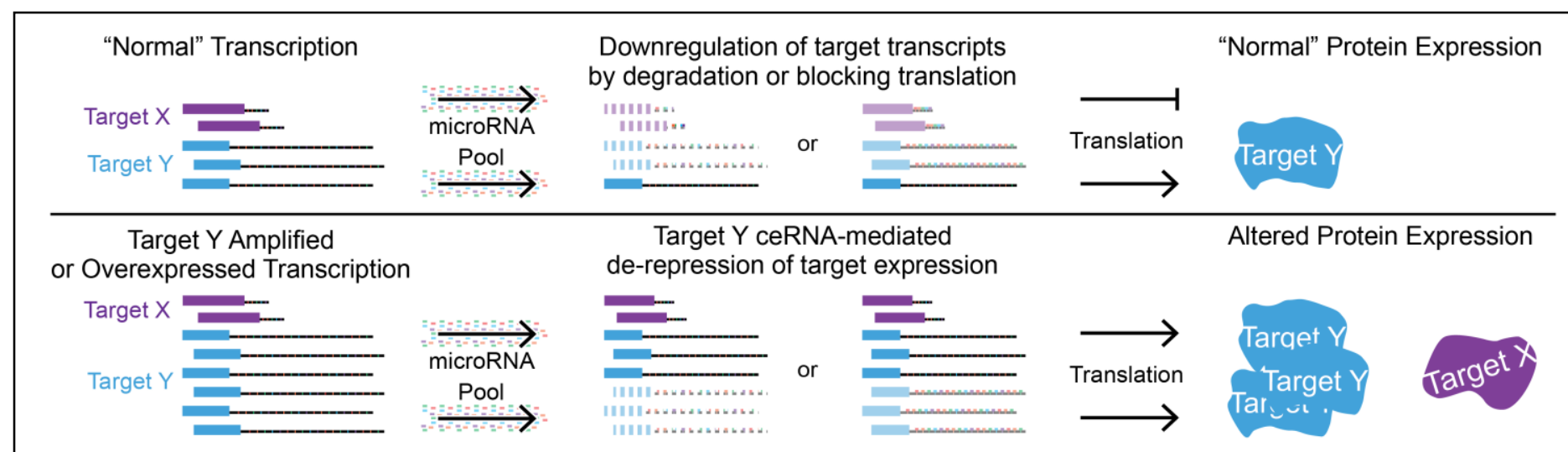

Figure 15. The ceRNA hypothesis at a glance.

Despite these conflicting reports, ceRNAs have been implicated in several disease states including cardiovascular disease with roles in atrial fibrillation, myocardial infarction, and cardiac hypertrophy(236-238); neurodegenerative disorders with roles in Parkinson's and Alzheimer's disease $(239,240)$; and cancer(241-245). It remains clear that additional studies are necessary to further bridge this gap of knowledge to either support or reject the ceRNA hypothesis. 


\section{Bibliography}

1. Chow LQM. Head and Neck Cancer. Longo DL, editor. N Engl J Med [Internet]. 2020 Jan 2 [cited 2020 May 4];382(1):60-72. Available from: http://www.nejm.org/doi/10.1056/NEJMra1715715

2. Siegel RL, Miller KD, Jemal A. Cancer statistics, 2020. CA Cancer J Clin [Internet]. 2020 Jan 8 [cited 2020 May 4];70(1):7-30. Available from: https://onlinelibrary.wiley.com/doi/abs/10.3322/caac.21590

3. Jethwa AR, Khariwala SS. Tobacco-related carcinogenesis in head and neck cancer. Cancer Metastasis Rev [Internet]. 2017 [cited 2020 Jul 14];36(3):411-23. Available from:

http://www.ncbi.nlm.nih.gov/pubmed/28801840

4. Ang KK, Harris J, Wheeler R, Weber R, Rosenthal DI, Nguyen-Tân PF, et al. Human papillomavirus and survival of patients with oropharyngeal cancer. N Engl J Med [Internet]. 2010 Jul 1;363(1):24-35.

Available from:

http://www.ncbi.nlm.nih.gov/pubmed/20530316\%0Ahttp://www.pubmedcentral.nih.gov/articlerender.fcgi ?artid=PMC2943767

5. National Comprehensive Cancer Network. Head and Neck Cancers (Version 2.2020) [Internet]. 2020. Available from: https://www.nccn.org/professionals/physician_gls/pdf/head-and-neck.pdf

6. Specenier P, Vermorken JB. Cetuximab in the treatment of squamous cell carcinoma of the head and neck. Expert Rev Anticancer Ther. 2011;11(4):511-24.

7. Burtness B, Harrington KJ, Greil R, Soulières D, Tahara M, de Castro G, et al. Pembrolizumab alone or with chemotherapy versus cetuximab with chemotherapy for recurrent or metastatic squamous cell carcinoma of the head and neck (KEYNOTE-048): a randomised, open-label, phase 3 study. Lancet. 2019;394(10212):1915-28.

8. Lawrence MS, Sougnez C, Lichtenstein L, Cibulskis K, Lander E, Gabriel SB, et al. Comprehensive genomic characterization of head and neck squamous cell carcinomas. Nature [Internet]. 2015;517(7536):576-82. Available from: http://www.nature.com/doifinder/10.1038/nature14129

9. Keck MK, Zuo Z, Khattri A, Stricker TP, Brown CD, Imanguli M, et al. Integrative Analysis of Head and 
Neck Cancer Identifies Two Biologically Distinct HPV and Three Non-HPV Subtypes. Clin Cancer Res

[Internet]. 2015;21(4):870-81. Available from: http://clincancerres.aacrjournals.org/cgi/doi/10.1158/1078-

0432.CCR-14-2481

10. Maitra A, Biswas NK, Amin K, Kowtal P, Kumar S, Das S, et al. Mutational landscape of gingivo-buccal oral squamous cell carcinoma reveals new recurrently-mutated genes and molecular subgroups. Nat Commun [Internet]. 2013 Dec 2 [cited 2019 Apr 6];4(1):2873. Available from:

http://www.nature.com/articles/ncomms3873

11. Stransky N, Egloff AM, Tward AD, Kostic AD, Sougnez C, Mckenna A, et al. The Mutational Landscape of Head Squamous Cell Carcinoma. Science (80- ). 2014;333(6046):1157-60.

12. Argiris A, Karamouzis M V, Raben D, Ferris RL. Head and neck cancer. Lancet (London, England) [Internet]. 2008;371(9625):1695-709. Available from:

http://www.sciencedirect.com/science/article/pii/S014067360860728X

13. Hayes DN, Van Waes C, Seiwert TY. Genetic landscape of human papillomavirus-associated head and neck cancer and comparison to tobacco-related tumors. J Clin Oncol [Internet]. 2015 Oct 10 [cited 2020 Jun 19];33(29):3227-34. Available from: http://www.ncbi.nlm.nih.gov/pubmed/26351353

14. Benson E, Li R, Eisele D, Fakhry C. The clinical impact of HPV tumor status upon head and neck squamous cell carcinomas. Oral Oncol [Internet]. 2014 Jun [cited 2020 Jun 29];50(6):565-74. Available from: http://www.ncbi.nlm.nih.gov/pubmed/24134947

15. Chaturvedi AK, Engels EA, Pfeiffer RM, Hernandez BY, Xiao W, Kim E, et al. Human papillomavirus and rising oropharyngeal cancer incidence in the United States. J Clin Oncol [Internet]. 2011 Nov 10 [cited 2017 Oct 20];29(32):4294-301. Available from: http://www.ncbi.nlm.nih.gov/pubmed/21969503

16. Rautava J, Syrjänen S. Biology of Human Papillomavirus Infections in Head and Neck Carcinogenesis [Internet]. Vol. 6, Head and Neck Pathology. Springer; 2012 [cited 2020 Jun 29]. p. 3-15. Available from: http://www.ncbi.nlm.nih.gov/pubmed/22782219

17. Gillison ML, Chaturvedi AK, Anderson WF, Fakhry C. Epidemiology of human papillomavirus-positive 
head and neck squamous cell carcinoma [Internet]. Vol. 33, Journal of Clinical Oncology. American Society of Clinical Oncology; 2015 [cited 2020 Jun 29]. p. 3235-42. Available from:

http://www.ncbi.nlm.nih.gov/pubmed/26351338

18. Gillison ML. Evidence for a Causal Association Between Human Papillomavirus and a Subset of Head and Neck Cancers. J Natl Cancer Inst. 2000;92(9):709-20.

19. Kim SH, Koo BS, Kang S, Park K, Kim H, Lee KR, et al. HPV integration begins in the tonsillar crypt and leads to the alteration of p16, EGFR and c-myc during tumor formation. Int J Cancer. 2007;120(7):141825.

20. Thavaraj S, Stokes A, Mazuno K, Henley-Smith R, Suh YE, Paleri V, et al. Patients with HPV-related tonsil squamous cell carcinoma rarely harbour oncogenic HPV infection at other pharyngeal sites. Oral Oncol [Internet]. 2014;50(4):241-6. Available from: http://dx.doi.org/10.1016/j.oraloncology.2013.12.012

21. Sano D, Oridate N. The molecular mechanism of human papillomavirus-induced carcinogenesis in head and neck squamous cell carcinoma [Internet]. Vol. 21, International Journal of Clinical Oncology.

Springer; 2016 [cited 2020 Jun 29]. p. 819-26. Available from: http://link.springer.com/10.1007/s10147016-1005-x

22. Drews CM, Brimer N, Vande Pol SB. Multiple regions of E6AP (UBE3A) contribute to interaction with papillomavirus E6 proteins and the activation of ubiquitin ligase activity. PLoS Pathog [Internet]. 2020 [cited $2020 \mathrm{Jul}$ 4];16(1):e1008295. Available from: http://www.ncbi.nlm.nih.gov/pubmed/31971989

23. Lewis JS, Thorstad WL, Chernock RD, Haughey BH, Yip JH, Zhang Q, et al. P16 positive oropharyngeal squamous cell carcinoma: An entity with a favorable prognosis regardless of tumor HPV status. Am J Surg Pathol [Internet]. 2010 Aug [cited 2020 Jun 29];34(8):1088-96. Available from: http://www.ncbi.nlm.nih.gov/pubmed/20588174

24. Oosthuizen JC, Kinsella JB. Is treatment de-escalation a reality in HPV related oropharyngeal cancer? Surgeon [Internet]. 2016;14(4):180-3. Available from: http://dx.doi.org/10.1016/j.surge.2016.04.002

25. White R, Abel S, Hasan S, Verma V, Greenberg L, Colonias A, et al. Practice patterns and outcomes 
following radiation dose de-escalation for oropharyngeal cancer. Laryngoscope. 2019;1-6.

26. Oosthuizen JC, Doody J. De-intensified treatment in human papillomavirus-positive oropharyngeal cancer. Lancet [Internet]. 2019 Jan;393(10166):5-7. Available from:

https://linkinghub.elsevier.com/retrieve/pii/S0140673618329301

27. Lydiatt WM, Patel SG, O'Sullivan B, Brandwein MS, Ridge JA, Migliacci JC, et al. Head and Neck cancers-major changes in the American Joint Committee on cancer eighth edition cancer staging manual. CA Cancer J Clin [Internet]. 2017;67(2):122-37. Available from:

http://www.ncbi.nlm.nih.gov/pubmed/28128848

28. Cheraghlou S, Yu PK, Otremba MD, Park HS, Bhatia A, Zogg CK, et al. Treatment deintensification in human papillomavirus-positive oropharynx cancer: Outcomes from the National Cancer Data Base. Cancer [Internet]. 2018 Feb 15 [cited 2020 Jun 29];124(4):717-26. Available from: http://doi.wiley.com/10.1002/cncr.31104

29. Saslow D, Andrews KS, Manassaram-Baptiste D, Loomer L, Lam KE, Fisher-Borne M, et al. Human papillomavirus vaccination guideline update: American Cancer Society guideline endorsement. CA Cancer J Clin [Internet]. 2016 Jul 8 [cited 2020 Jul 8];66(5):375-85. Available from: https://onlinelibrary.wiley.com/doi/abs/10.3322/caac.21616

30. Mroz EA, Tward AD, Pickering CR, Myers JN, Ferris RL, Rocco JW. High intratumor genetic heterogeneity is related to worse outcome in patients with head and neck squamous cell carcinoma. Cancer. 2013;119(16):3034-42.

31. Hammerman PS, Neil Hayes D, Grandis JR. Therapeutic insights from genomic studies of head and neck squamous cell carcinomas. Cancer Discov. 2015;5(3):239-44.

32. Wee P, Wang Z. Epidermal growth factor receptor cell proliferation signaling pathways [Internet]. Vol. 9, Cancers. Multidisciplinary Digital Publishing Institute (MDPI); 2017 [cited 2020 Jul 4]. Available from: https://www.ncbi.nlm.nih.gov/pmc/articles/PMC5447962/

33. Campisi J, Gray HE, Pardee AB, Dean M, Sonenshein GE. Cell-cycle control of c-myc but not c-ras 
expression is lost following chemical transformation. Cell. 1984;36(2):241-7.

34. Kato S, Okamura R, Mareboina M, Lee S, Goodman A, Patel SP, et al. Revisiting Epidermal Growth Factor Receptor ( EGFR ) Amplification as a Target for Anti-EGFR Therapy: Analysis of Cell-Free Circulating Tumor DNA in Patients With Advanced Malignancies. JCO Precis Oncol [Internet]. 2019 [cited 2020 Jul 5];3(3):1-14. Available from: https://www.ncbi.nIm.nih.gov/pmc/articles/PMC6497417/

35. Sullivan KD, Galbraith MD, Andrysik Z, Espinosa JM. Mechanisms of transcriptional regulation by p53 [Internet]. Vol. 25, Cell Death and Differentiation. Nature Publishing Group; 2018 [cited 2020 Jul 5]. p. 133-43. Available from: http://www.ncbi.nlm.nih.gov/pubmed/29125602

36. Job S, De Reyniès A, Heller B, Weiss A, Guérin E, Macabre C, et al. Preferential response of basal-like head and neck squamous cell carcinoma cell lines to EGFR-Targeted therapy depending on EREGDriven oncogenic addiction. Cancers (Basel) [Internet]. 2019 Jun 8 [cited 2020 Jul 6];11(6). Available from: http://www.ncbi.nlm.nih.gov/pubmed/31181806

37. Knox S, Basu S, Remick S. A Systems Approach to Cancer Health Disparities in Appalachia. Austin J Public Heal Epidemiol. 2014;1(1):1004.

38. Wilson RJ, Ryerson AB, Singh SD, King JB. Cancer Incidence in Appalachia, 2004-2011. Cancer Epidemiol Biomarkers Prev [Internet]. 2016 Feb [cited 2016 Mar 8];25(February):2004-11. Available from: http://www.ncbi.nlm.nih.gov/pubmed/26819264

39. Lengerich EJ, Lehman E, Siedlecki JC, Tucker TC, Wyatt SW, Powell RK, et al. Cancer incidence in Kentucky, Pennsylvania, and West Virginia: Disparities in Appalachia. J Rural Heal. 2004;21(1):39-47.

40. Wingo PA, Tucker TC, Jamison PM, Martin H, McLaughlin C, Bayakly R, et al. Cancer in Appalachia, 2001-2003. Cancer [Internet]. 2008 Jan 1 [cited 2020 Jun 30];112(1):181-92. Available from: http://doi.wiley.com/10.1002/cncr.23132

41. Friedell GH, Rubio A, Maretzki A, Garland B, Brown P, Crane M, et al. Community cancer control in a rural, underserved population: the Appalachian Leadership Initiative on Cancer Project. J Health Care Poor Underserved [Internet]. 2001 Feb;12(1):5-19. Available from: 
http://www.ncbi.nlm.nih.gov/pubmed/11217227

42. Appalachian Regional Development. 40 U.S.C § 14102(a)(1).

43. County Economic Status in Appalachia, FY 2017 - Appalachian Regional Commission [Internet]. [cited 2019 Aug 4]. Available from: https://www.arc.gov/research/MapsofAppalachia.asp?MAP_ID=116

44. Pollard K, Jacobsen LA. The Appalachian Region: A Data Overview from the 2013-2017 American Community Survey - Appalachian Regional Commission [Internet]. 2019 [cited 2019 Aug 4]. Available from: https://www.arc.gov/research/researchreportdetails.asp?REPORT_ID=159

45. Viens LJ, Jane Henley S, Watson M, Markowitz LE, Thomas CC, Thompson TD, et al. Human papillomavirus-associated cancers — United States, 2008-2012. Morb Mortal Wkly Rep [Internet]. 2016 Jul 8 [cited 2019 Aug 4];65(26):661-6. Available from:

http://www.cdc.gov/mmwr/volumes/65/wr/mm6526a1.htm

46. Behavioral Risk Factor Data: Tobacco Use (2011 to present) | Chronic Disease and Health Promotion Data \&amp; Indicators [Internet]. [cited 2019 Aug 4]. Available from: https://chronicdata.cdc.gov/SurveyData/Behavioral-Risk-Factor-Data-Tobacco-Use-2011-to-pr/wsas-xwh5

47. Reiter PL, Fisher JL, Hudson AG, Tucker TC, Plascak JJ, Paskett ED. Assessing the burden of HPVrelated cancers in Appalachia. Hum Vaccines Immunother. 2013;9(1):90-6.

48. Bruce C. Casto, Smita Sharma, James L. Fisher, Thomas J. Knobloch, Amit Agrawal, Christopher M. Weghorst. Oral Cancer in Appalachia. J Health Care Poor Underserved. 2009;20(1):274-85.

49. Ringstrom MJ, Christian J, Bush ML, Levy JE, Huang B, Gal TJ. Travel distance: Impact on stage of presentation and treatment choices in head and neck cancer. Am J Otolaryngol - Head Neck Med Surg. 2018;39(5):575-81.

50. Gleich LL, Collins CM, Gartside PS, Gluckman JL, Barrett WL, Wilson KM, et al. Therapeutic Decision Making in Stages III and IV Head and Neck Squamous Cell Carcinoma. Arch Otolaryngol Neck Surg [Internet]. 2003 Jan 1;129(1):26. Available from:

http://archotol.jamanetwork.com/article.aspx?doi=10.1001/archotol.129.1.26 
51. Stanford-Moore G, Bradshaw PT, Weissler MC, Zevallos JP, Brennan P, Anantharaman D, et al. Interaction between known risk factors for head and neck cancer and socioeconomic status: the Carolina Head and Neck Cancer Study. Cancer Causes Control [Internet]. 2018;29(9):863-73. Available from: http://dx.doi.org/10.1007/s10552-018-1062-8

52. Welte JW, Barnes GM, Tidwell MCO, Hoffman JH. Tobacco use, heavy use, and dependence among adolescents and young adults in the United States. Subst Use Misuse. 2011;46(9):1090-8.

53. Nemeth JM, Liu ST, Klein EG, Ferketich AK, Kwan MP, Wewers ME. Factors influencing smokeless tobacco use in rural Ohio Appalachia. J Community Health. 2012;37(6):1208-17.

54. Proctor RN. The history of the discovery of the cigaretteelung cancer link: Evidentiary traditions, corporate denial, global toll. Tob Control [Internet]. 2012 Mar 1 [cited 2020 Jul 1];21(2):87-91. Available from: https://tobaccocontrol.bmj.com/content/21/2/87.full

55. Doll R, Hill AB. The mortality of doctors in relation to their smoking habits: a preliminary report: (Reprinted from Br Med J 1954:ii;1451-5). BMJ [Internet]. 1954 [cited 2020 Jul 1];328(7455):1529-33. Available from: https://www.ncbi.nlm.nih.gov/pmc/articles/PMC437141/

56. U.S. Department of Health and Human Services. The Health Consequences of Smoking-50 Years of Progress. Med Clin North Am. 2014;

57. Steppuhn A, Gase K, Krock B, Halitschke R, Baldwin IT. Nicotine's defensive function in nature. PLoS Biol [Internet]. 2004 [cited 2020 Jul 1];2(8). Available from:

https://www.ncbi.nlm.nih.gov/pmc/articles/PMC509292/

58. Brody AL, Mandelkern MA, London ED, Olmstead RE, Farahi J, Scheibal D, et al. Cigarette smoking saturates brain $\alpha 4 \beta 2$ nicotinic acetylcholine receptors. Arch Gen Psychiatry [Internet]. 2006 [cited 2020 Jul 1];63(8):907-15. Available from: https://www.ncbi.nlm.nih.gov/pmc/articles/PMC2773659/

59. United States Environmental Protection Agency. Ambient Water Quality Criteria for Polynuclear Aromatic Hydrocarbons. 1980. p. 1-165.

60. WHO IARC. IARC Monographs. On the evaluation of the carcinogenic risks to humans. Overall 
evaluations of carcinogenicity: An updating of IARC monographs Volumes 1 to 42. WHO Int Agency Res Cancer Monogr. 1987;1-42.

61. Ding YS, Yan XJ, Jain RB, Lopp E, Tavakoli A, Polzin GM, et al. Determination of 14 polycyclic aromatic hydrocarbons in mainstream smoke from U.S. brand and non-U.S. brand cigarettes. Environ Sci Technol. 2006;40(4):1133-8.

62. U.S. Department of Health and Human Services. How Tobacco Smoke Causes Disease: The Biology and Behavioral Basis for Smoking-Attributable Disease. How Tobacco Smoke Causes Disease: The Biology and Behavioral Basis for Smoking-Attributable Disease: A Report of the Surgeon General. 2010. $792 \mathrm{p}$.

63. Waalkes MP. Cadmium carcinogenesis in review. J Inorg Biochem [Internet]. 2000 Apr 30 [cited 2020 Jul 1];79(1-4):241-4. Available from:

https://www.sciencedirect.com/science/article/pii/S016201340000009X?via\%3Dihub

64. Rickert WS, Kaiserman MJ. Levels of Lead, Cadmium, and Mercury in Canadian Cigarette Tobacco as Indicators of Environmental Change: Results from a 21-Year Study (1968-1988). Environ Sci Technol. 1994;28(5):924-7.

65. Counts ME, Morton MJ, Laffoon SW, Cox RH, Lipowicz PJ. Smoke composition and predicting relationships for international commercial cigarettes smoked with three machine-smoking conditions. Regul Toxicol Pharmacol. 2005;41(3):185-227.

66. Coleman W, Perfetti T. The Roles of Amino Acids and Sugars in the Production of Volatile Materials in Microwave Heated Tobacco Dust Suspensions. Beiträge zur Tab Int to Tob Res. 2016;17(3):75-95.

67. WHO IARC. IARC monographs. On the evaluation of carcinogenic risks to humans. Re-evaluation of some organic chemicals, hydrazine, and hydrozen peroxide. WHO Int Agency Res Cancer Monogr. $1999 ; 71$.

68. Patrianakos C, Hoffmann D. Chemical studies on tobacco smoke LXIV. On the analysis of aromatic amines in cigarette smoke. J Anal Toxicol [Internet]. 1979 Jul 1 [cited 2020 Jul 1];3(4):150-4. Available 
from: http://academic.oup.com/jat/article/3/4/150/686605/Chemical-Studies-on-Tobacco-Smoke-LXIV-

On-the

69. Radomski JL, Brill E. Bladder cancer induction by aromatic amines: Role of N-hydroxy metabolites.

Science (80- ) [Internet]. 1970 Feb 13 [cited 2020 Jul 1];167(3920):992-3. Available from:

https://science.sciencemag.org/content/167/3920/992.abstract

70. WHO IARC. IARC Monographs. On the evaluation of the carcinogenic risk of chemicals to humans. Tobacco smoking. WHO Int Agency Res Cancer Monogr. 1986;38.

71. Brown BG, Borschke AJ, Doolittle DJ. An Analysis of the Role of Tobacco-Specific Nitrosamines in the Carcinogenicity of Tobacco Smoke. Nonlinearity Biol Toxicol Med [Internet]. 2003 [cited 2020 Jul 1];1(2):154014203914343. Available from: https://www.ncbi.nlm.nih.gov/pmc/articles/PMC2651603/

72. WHO IARC. IARC Monographs on the Evaluation of Carcinogenic Risks to Humans; Smokeless Tobacco and Some Tobacco-specific N -Nitrosamines Smokeless Tobacco and Some. WHO Int Agency Res Cancer Monogr. 2007;89.

73. Lee PN, Hamling J. Systematic review of the relation between smokeless tobacco and cancer in Europe and North America. BMC Med [Internet]. 2009 Jul 29 [cited 2020 Jun 30];7:36. Available from: http://www.ncbi.nlm.nih.gov/pubmed/19638245

74. Farsalinos KE, Gillman G, Poulas K, Voudris V. Tobacco-Specific Nitrosamines in Electronic Cigarettes: Comparison between liquid and aerosol levels. Int J Environ Res Public Health [Internet]. 2015 [cited 2020 Jul 1];12(8):9046-53. Available from: https:/www.ncbi.nlm.nih.gov/pmc/articles/PMC4555263/

75. Kim HJ, Shin HS. Determination of tobacco-specific nitrosamines in replacement liquids of electronic cigarettes by liquid chromatography-tandem mass spectrometry. J Chromatogr A [Internet]. 2013;1291:48-55. Available from: http://dx.doi.org/10.1016/j.chroma.2013.03.035

76. Cummings KM, Dresler CM, Field JK, Fox J, Gritz ER, Hanna NH, et al. E-cigarettes and cancer patients. J Thorac Oncol [Internet]. 2014;9(4):438-41. Available from: http://dx.doi.org/10.1097/JTO.0000000000000129 
77. Kato S, Bowman ED, Harrington AM, Blomeke B, Shields PG. Human lung carcinogen-dna adduct levels mediated by genetic polymorphisms in vivo. J Natl Cancer Inst [Internet]. 1995 Jun 21 [cited 2020 Jul 2];87(12):902-7. Available from: https://academic.oup.com/jnci/articlelookup/doi/10.1093/jnci/87.12.902

78. WHO IARC. IARC Monographs. On the evaluation of the carcinogenic risk of chemicals to humans. Polynuclear aromatic compounds, part 1, chemical, environmental, and experimental data. WHO Int Agency Res Cancer Monogr. 1983;32.

79. Hecht SS. Biochemistry, biology, and carcinogenicity of tobacco-specific N- nitrosamines [Internet]. Vol. 11, Chemical Research in Toxicology. Chem Res Toxicol; 1998 [cited 2020 Jul 2]. p. 559-603. Available from: https://pubmed.ncbi.nlm.nih.gov/9625726/

80. Chatterjee N, Walker GC. Mechanisms of DNA damage, repair, and mutagenesis [Internet]. Vol. 58, Environmental and Molecular Mutagenesis. NIH Public Access; 2017 [cited 2020 Jul 2]. p. 235-63. Available from: https://www.ncbi.nlm.nih.gov/pmc/articles/PMC5474181/

81. DeMarini DM. Genotoxicity of tobacco smoke and tobacco smoke condensate: A review [Internet]. Vol. 567, Mutation Research - Reviews in Mutation Research. Elsevier; 2004 [cited 2020 Jul 2]. p. 447-74. Available from: https://www.sciencedirect.com/science/article/pii/S138357420400064X?via\%3Dihub

82. Pfeiffer P, Goedecke W, Obe G. Mechanisms of DNA double-strand break repair and their potential to induce chromosomal aberrations. Mutagenesis [Internet]. 2000 Jul 1 [cited 2020 Jul 2];15(4):289-302. Available from: https://academic.oup.com/mutage/article-lookup/doi/10.1093/mutage/15.4.289

83. Williams AB, Schumacher B. p53 in the DNA-damage-repair process. Cold Spring Harb Perspect Med [Internet]. 2016 [cited 2020 Jul 2];6(5). Available from: https://www.ncbi.nlm.nih.gov/pmc/articles/PMC4852800/

84. Surget S, Khoury MP, Bourdon JC. Uncovering the role of p53 splice variants in human malignancy: A clinical perspective [Internet]. Vol. 7, OncoTargets and Therapy. Dove Press; 2013 [cited 2020 Jul 2]. p. 57-67. Available from: https://www.ncbi.nlm.nih.gov/pmc/articles/PMC3872270/ 
85. Hanel W, Moll UM. Links between mutant p53 and genomic instability. J Cell Biochem. $2012 ; 113(2): 433-9$.

86. Menon V, Povirk L. Involvement of p53 in the Repair of DNA Double Strand Breaks: Multifaceted Roles of p53 in Homologous Recombination Repair (HRR) and Non-Homologous End Joining (NHEJ). In: Mutant p53 and MDM2 in Cancer [Internet]. 2014. p. 321-36. Available from: http://link.springer.com/10.1007/978-94-017-9211-0

87. Hanahan D, Weinberg RA. Hallmarks of cancer: The next generation. Cell [Internet]. 2011 Mar 4 [cited 2020 Jul 2];144(5):646-74. Available from: https://www.cell.com/cell/fulltext/S0092-8674(11)001279?_returnURL=https\%3A\%2F\%2Flinkinghub.elsevier.com\%2Fretrieve\%2Fpii\%2FS0092867411001279 \%3Fshowall\%3Dtrue

88. Holland AJ, Cleveland DW. Boveri revisited: Chromosomal instability, aneuploidy and tumorigenesis [Internet]. Vol. 10, Nature Reviews Molecular Cell Biology. NIH Public Access; 2009 [cited 2020 Jul 2]. p. 478-87. Available from: https://www.ncbi.nlm.nih.gov/pmc/articles/PMC3154738/

89. Mermel CH, Schumacher SE, Hill B, Meyerson ML, Beroukhim R, Getz G. GISTIC2.0 facilitates sensitive and confident localization of the targets of focal somatic copy-number alteration in human cancers. Genome Biol [Internet]. 2011 [cited 2020 Jun 19];12(4):R41. Available from: http://www.ncbi.nlm.nih.gov/pubmed/21527027

90. Tijhuis AE, Johnson SC, McClelland SE. The emerging links between chromosomal instability (CIN), metastasis, inflammation and tumour immunity. Mol Cytogenet [Internet]. 2019 [cited 2020 Jul 2];12(1). Available from: https://www.ncbi.nlm.nih.gov/pmc/articles/PMC6518824/

91. Lee AJX, Endesfelder D, Rowan AJ, Walther A, Birkbak NJ, Futreal PA, et al. Chromosomal instability confers intrinsic multidrug resistance. Cancer Res [Internet]. 2011 [cited 2020 Jul 2];71(5):1858-70. Available from: https://www.ncbi.nlm.nih.gov/pmc/articles/PMC3059493/

92. Kikutake C, Yoshihara M, Sato T, Saito D, Suyama M. Pan-cancer analysis of intratumor heterogeneity associated with patient prognosis using multidimensional measures. Oncotarget [Internet]. 2018 [cited 
2020 Jul 2];9(102):37689-99. Available from: https://www.ncbi.nlm.nih.gov/pmc/articles/PMC6340877/

93. Janiszewska M. The microcosmos of intratumor heterogeneity: the space-time of cancer evolution [Internet]. Vol. 39, Oncogene. Nature Publishing Group; 2020 [cited 2020 Jul 2]. p. 2031-9. Available from: http://www.nature.com/articles/s41388-019-1127-5

94. Brosens RP, Haan JC, Carvalho B, Rustenburg F, Grabsch H, Quirke P, et al. Candidate driver genes in focal chromosomal aberrations of stage II colon cancer. J Pathol [Internet]. 2010 Apr 23 [cited 2020 Jul 2];221(4):411-24. Available from: http://doi.wiley.com/10.1002/path.2724

95. Koboldt DC, Zhang Q, Larson DE, Shen D, McLellan MD, Lin L, et al. VarScan 2: Somatic mutation and copy number alteration discovery in cancer by exome sequencing. Genome Res [Internet]. 2012 Mar 1 [cited 2020 Jul 2];22(3):568-76. Available from: https://genome.cshlp.org/content/22/3/568

96. Beroukhim R, Mermel CH, Porter D, Wei G, Raychaudhuri S, Donovan J, et al. The landscape of somatic copy-number alteration across human cancers. Nature [Internet]. 2010 [cited 2020 Jul 2];463(7283):899-905. Available from: https://www.ncbi.nlm.nih.gov/pmc/articles/PMC2826709/

97. Zack TI, Schumacher SE, Carter SL, Cherniack AD, Saksena G, Tabak B, et al. Pan-cancer patterns of somatic copy number alteration. Nat Genet [Internet]. 2013 Oct 26 [cited 2020 Jul 2];45(10):1134-40. Available from: http://www.nature.com/articles/ng.2760

98. O'Connell P, Cawthon R, Xu GF, Li Y, Viskochil D, White R. The neurofibromatosis type 1 (NF1) gene: identification and partial characterization of a putative tumor suppressor gene. In: Journal of Dermatology [Internet]. John Wiley \& Sons, Ltd; 1992 [cited 2020 Jul 5]. p. 881-4. Available from: http://doi.wiley.com/10.1111/j.1346-8138.1992.tb03799.x

99. Yamaguchi T, Hosono Y, Yanagisawa K, Takahashi T. NKX2-1/TTF-1: An Enigmatic Oncogene that Functions as a Double-Edged Sword for Cancer Cell Survival and Progression [Internet]. Vol. 23, Cancer Cell. Elsevier; 2013 [cited 2020 Jul 5]. p. 718-23. Available from: https://linkinghub.elsevier.com/retrieve/pii/S1535610813001360

100. Momand J, Zambetti GP, Olson DC, George D, Levine AJ. The mdm-2 oncogene product forms a 
complex with the p53 protein and inhibits p53-mediated transactivation. Cell. 1992;69(7):1237-45.

101. Oliner JD, Kinzler KW, Meltzer PS, George DL, Vogelstein B. Amplification of a gene encoding a p53associated protein in human sarcomas. Vol. 358, Nature. 1992. p. 80-3.

102. Irony-Tur Sinai M, Kerem B. Genomic instability in fragile sites—still adding the pieces. Genes Chromosom Cancer [Internet]. 2019 May 1 [cited 2020 Jul 2];58(5):295-304. Available from: http://doi.wiley.com/10.1002/gcc.22715

103. Debatisse M, Rosselli F. A journey with common fragile sites: From S phase to telophase. Genes Chromosom Cancer [Internet]. 2019 May 1 [cited 2020 Jul 2];58(5):305-16. Available from: http://doi.wiley.com/10.1002/gcc.22704

104. Smith DI, Zhu Y, McAvoy S, Kuhn R. Common fragile sites, extremely large genes, neural development and cancer. In: Cancer Letters [Internet]. Elsevier; 2006 [cited 2020 Jul 2]. p. 48-57. Available from: https://www.sciencedirect.com/science/article/abs/pii/S0304383505008232?via\%3Dihub

105. Hellman A, Zlotorynski E, Scherer SW, Cheung J, Vincent JB, Smith DI, et al. A role for common fragile site induction in amplification of human oncogenes. Cancer Cell. 2002;1(1):89-97.

106. Sutherland GR, Baker E, Richards RI. Fragile sites still breaking. Trends Genet. 1998;14(12):501-6.

107. Kaushal S, Freudenreich $\mathrm{CH}$. The role of fork stalling and DNA structures in causing chromosome fragility [Internet]. Vol. 58, Genes Chromosomes and Cancer. NIH Public Access; 2019 [cited 2020 Jul 2]. p. 270-83. Available from: https://www.ncbi.nlm.nih.gov/pmc/articles/PMC7083089/

108. Minca EC, Kowalski D. Replication fork stalling by bulky DNA damage: Localization at active origins and checkpoint modulation. Nucleic Acids Res [Internet]. 2011 [cited 2020 Jul 2];39(7):2610-23. Available from: https://www.ncbi.nlm.nih.gov/pmc/articles/PMC3074140/

109. Narayanan V, Mieczkowski PA, Kim H-M, Petes TD, Lobachev KS. The Pattern of Gene Amplification Is Determined by the Chromosomal Location of Hairpin-Capped Breaks. Cell [Internet]. 2006 Jun [cited 2020 Jul 2];125(7):1283-96. Available from: https://pubmed.ncbi.nlm.nih.gov/16814715/ 
110. Coquelle A, Pipiras E, Toledo F, Buttin G, Debatisse M. Expression of fragile sites triggers intrachromosomal mammalian gene amplification and sets boundaries to early amplicons. Cell [Internet]. 1997 [cited 2020 Jul 2];89(2):215-25. Available from: https://pubmed.ncbi.nlm.nih.gov/9108477/

111. McClintock B. The Stability of Broken Ends of Chromosomes in Zea Mays. Genetics. 1941;

112. Gillison ML, Akagi K, Xiao W, Jiang B, Pickard RKL, Li J, et al. Human papillomavirus and the landscape of secondary genetic alterations in oral cancers. Genome Res [Internet]. 2019;29(1):1-17. Available from: http://www.ncbi.nlm.nih.gov/pubmed/30563911

113. Gollin SM. Cytogenetic Alterations and their Molecular Genetic Correlates in Head and Neck Squamous Cell Carcinoma: A Next Generation Window to the Biology of Disease. Genes Chromosomes Cancer [Internet]. 2014 Dec [cited 2020 Jul 20];53(12):972-90. Available from: https://pubmed.ncbi.nlm.nih.gov/25183546/

114. Cheng H, Yang X, Si H, Saleh AD, Xiao W, Coupar J, et al. Genomic and Transcriptomic Characterization Links Cell Lines with Aggressive Head and Neck Cancers. Cell Rep [Internet]. 2018 [cited 2020 Jul 20];25(5):1332-1345.e5. Available from: https://pubmed.ncbi.nlm.nih.gov/30380422/

115. Hermida-Prado F, Menéndez ST, Albornoz-Afanasiev P, Granda-Diaz R, Álvarez-Teijeiro S, Villaronga MÁ, et al. Distinctive Expression and Amplification of Genes at 11q13 in Relation to HPV Status with Impact on Survival in Head and Neck Cancer Patients. J Clin Med [Internet]. 2018 Dec 1;7(12):501. Available from: http://www.ncbi.nlm.nih.gov/pubmed/30513772

116. Seiwert TY, Zuo Z, Keck MK, Khattri A, Pedamallu CS, Stricker T, et al. Integrative and comparative genomic analysis of HPV-positive and HPV-negative head and neck squamous cell carcinomas. Clin Cancer Res [Internet]. 2015 Feb 1 [cited 2020 Jun 19];21(3):632-41. Available from: http://www.ncbi.nlm.nih.gov/pubmed/25056374

117. Dogan S, Xu B, Middha S, Vanderbilt CM, Bowman AS, Migliacci J, et al. Identification of prognostic molecular biomarkers in 157 HPV-positive and HPV-negative squamous cell carcinomas of the oropharynx. Int J Cancer [Internet]. 2019 Dec 30 [cited 2020 Jun 19];145(11):3152-62. Available from: 
https://onlinelibrary.wiley.com/doi/abs/10.1002/ijc.32412

118. Huang X, Gollin SM, Raja S, Godfrey TE. High-resolution mapping of the 11q13 amplicon and identification of a gene, TAOS1, that is amplified and overexpressed in oral cancer cells. Proc Natl Acad Sci U S A [Internet]. 2002 Aug 20;99(17):11369-74. Available from:

http://www.pubmedcentral.nih.gov/articlerender.fcgi $?$ artid=123263\&tool=pmcentrez\&rendertype=abstrac t

119. Wilkerson PM, Reis-Filho JS. The 11q13-q14 amplicon: clinicopathological correlations and potential drivers. Genes Chromosomes Cancer [Internet]. 2013 Apr [cited 2014 Jun 3];52(4):333-55. Available from: http://onlinelibrary.wiley.com/doi/10.1002/gcc.22037/full

120. Reshmi SC, Roychoudhury S, Yu Z, Feingold E, Potter D, Saunders WS, et al. Inverted duplication pattern in anaphase bridges confirms the breakage-fusion-bridge (BFB) cycle model for 11q13 amplification. Cytogenet Genome Res. 2007;116(1-2):46-52.

121. Reshmi SC, Huang X, Schoppy DW, Black RC, Saunders WS, Smith DI, et al. Relationship between FRA11F and 11q13 gene amplification in oral cancer. Genes Chromosom Cancer. 2007;46(2):143-54.

122. Kao-Shan C-S, Fine RL, Whang-Peng J, Lee EC, Chabner BA, Lee EC. Increased Fragile Sites and Sister Chromatid Exchanges in Bone Marrow and Peripheral Blood of Young Cigarette Smokers. Cancer Res [Internet]. 1987;47(23):6278-82. Available from:

http://cancerres.aacrjournals.org/content/47/23/6278.abstract

123. Gibcus JH, Kok K, Menkema L, Hermsen MA, Mastik M, Kluin PM, et al. High-resolution mapping identifies a commonly amplified 11 q13.3 region containing multiple genes flanked by segmental duplications. Hum Genet. 2007;121(2):187-201.

124. Baldin V, Lukas J, Marcote MJ, Pagano M, Draetta G. Cyclin D1 is a nuclear protein required for cell cycle progression in G1. Genes Dev. 1993;7(5):812-21.

125. Izzo JG, Papadimitrakopoulou VA, Li XQ, Ibarguen H, Lee JS, Ro JY, et al. Dysregulated cyclin D1 expression early in head and neck tumorigenesis: in vivo evidence for an association with subsequent 
gene amplification. Oncogene [Internet]. 1998;17(18):2313-22. Available from:

http://www.ncbi.nlm.nih.gov/pubmed/9811462

126. Zhai C, Li Y, Mascarenhas C, Lin Q, Li K, Vyrides I, et al. The function of ORAOV1/LTO1, a gene that is overexpressed frequently in cancer: Essential roles in the function and biogenesis of the ribosome.

Oncogene [Internet]. 2014 Jan 14 [cited 2020 Jul 2];33(4):484-94. Available from:

http://www.nature.com/articles/onc2012604

127. Li M, Cui X, Shen Y, Dong H, Liang W, Chen Y, et al. ORAOV1 overexpression in esophageal squamous cell carcinoma and esophageal dysplasia: A possible biomarker of progression and poor prognosis in esophageal carcinoma. Hum Pathol [Internet]. 2015;46(5):707-15. Available from: http://dx.doi.org/10.1016/j.humpath.2015.01.009

128. Astro V, Asperti C, Cangi G, Doglioni C, de Curtis I. Liprin- $\alpha 1$ regulates breast cancer cell invasion by affecting cell motility, invadopodia and extracellular matrix degradation. Oncogene [Internet]. 2011;30(15):1841-9. Available from: http://www.nature.com/doifinder/10.1038/onc.2010.562

129. Asperti C, Astro V, Totaro A, Paris S, de Curtis I. Liprin-a1 promotes cell spreading on the extracellular matrix by affecting the distribution of activated integrins. J Cell Sci [Internet]. 2009;122(18):3225-32. Available from: http://jcs.biologists.org/cgi/doi/10.1242/jcs.054155

130. Pehkonen H, Lento M, Von Nandelstadh P, Filippou A, Grénman R, Lehti K, et al. Liprin- $\alpha 1$ modulates cancer cell signaling by transmembrane protein CD82 in adhesive membrane domains linked to cytoskeleton. Cell Commun Signal. 2018;16(1):1-14.

131. Godse NR, Khan N, Yochum ZA, Gomez-Casal R, Kemp C, Shiwarski DJ, et al. TMEM16A/ANO1 inhibits apoptosis via downregulation of Bim expression. Clin Cancer Res. 2017;23(23):7324-32.

132. Kischkel FC, Hellbardt S, Behrmann I, Germer M, Pawlita M, Krammer PH, et al. Cytotoxicity-dependent APO-1 (Fas/CD95)-associated proteins form a death-inducing signaling complex (DISC) with the receptor. EMBO J. 1995;14(22):5579-88.

133. Rasamny JJ, Allak A, Krook KA, Jo VY, Policarpio-Nicolas ML, Sumner HM, et al. Cyclin D1 and FADD 
as biomarkers in head and neck squamous cell carcinoma. Otolaryngol Head Neck Surg [Internet]. 2012 Jun;146(6):923-31. Available from: http://www.ncbi.nlm.nih.gov/pubmed/22323434

134. Pattje WJ, Melchers LJ, Slagter-Menkema L, Mastik MF, Schrijvers ML, Gibcus JH, et al. FADD expression is associated with regional and distant metastasis in squamous cell carcinoma of the head and neck. Histopathology. 2013;63(2):263-70.

135. Deng C, Wynshaw-Boris A, Zhou F, Kuo A, Leder P. Fibroblast growth factor receptor 3 is a negative regulator of bone growth. Cell [Internet]. 1996 Mar 22 [cited 2020 Jul 7];84(6):911-21. Available from: https://www.cell.com/cell/fulltext/S0092-8674(00)81069-

7?_returnURL=https\%3A\%2F\%2Flinkinghub.elsevier.com\%2Fretrieve\%2Fpii\%2FS0092867400810697 \%3Fshowall\%3Dtrue

136. Fioravanti L, Cappelletti V, Coradini D, Miodini P, Borsani G, Daidone MG, et al. int-2 oncogene amplification and prognosis in node-negative breast carcinoma. Int J Cancer [Internet]. 1997 [cited 2020 Jul 7];74(6):620-4. Available from: https://pubmed.ncbi.nlm.nih.gov/9421359/

137. Feldman B, Poueymirou W, Papaioannou VE, DeChiara TM, Goldfarb M. Requirement of FGF-4 for postimplantatior mouse development. Science (80- ) [Internet]. 1995 Jan 13 [cited 2020 Jul 7];267(5195):246-9. Available from: http://www.ncbi.nlm.nih.gov/pubmed/7809630

138. Ornitz DM, Itoh N. The fibroblast growth factor signaling pathway. Wiley Interdiscip Rev Dev Biol [Internet]. 2015 [cited $2020 \mathrm{Jul}$ 7];4(3):215-66. Available from:

http://www.ncbi.nlm.nih.gov/pubmed/25772309

139. Gao L, Lang L, Zhao X, Shay C, Shull AY, Teng Y. FGF19 amplification reveals an oncogenic dependency upon autocrine FGF19/FGFR4 signaling in head and neck squamous cell carcinoma. Oncogene [Internet]. 2019 [cited 2020 Jun 19];38(13):2394-404. Available from: https://pubmed.ncbi.nlm.nih.gov/30518874/

140. Ammer AG, Weed SA. Cortactin Branches Out: Roles in Regulating Protrusive Actin Dynamics [Internet]. Vol. 65, Cell Motility and the Cytoskeleton. NIH Public Access; 2009 [cited 2020 Jul 2]. 687- 
707 p. Available from: https://www.ncbi.nlm.nih.gov/pmc/articles/PMC2561250/

141. Markwell SM, Ammer AG, Interval ET, Allen JL, Papenberg BW, Hames RA, et al. Cortactin phosphorylation by casein kinase 2 regulates actin-related protein 2/3 complex activity, invadopodia function, and tumor cell invasion. Mol Cancer Res [Internet]. 2019 [cited 2020 Jul 2];17(4):987-1001. Available from: https://www.ncbi.nlm.nih.gov/pmc/articles/PMC6445698/

142. Timpson P, Wilson AS, Lehrbach GM, Sutherland RL, Musgrove EA, Daly RJ. Aberrant expression of cortactin in head and neck squamous cell carcinoma cells is associated with enhanced cell proliferation and resistance to the epidermal growth factor receptor inhibitor gefitinib. Cancer Res. 2007;67(19):9304-14.

143. Moreaux J, Hose D, Bonnefond A, Reme T, Robert N, Goldschmidt H, et al. MYEOV is a prognostic factor in multiple myeloma. Exp Hematol [Internet]. 2010 Dec [cited 2020 Jul 7];38(12):1189-1198.e3. Available from: http://www.ncbi.nlm.nih.gov/pubmed/20854874

144. Lawlor G, Doran PP, MacMathuna P, Murray DW. MYEOV (myeloma overexpressed gene) drives colon cancer cell migration and is regulated by PGE2. J Exp Clin Cancer Res [Internet]. 2010 [cited 2020 Jul 7];29(1):81. Available from: https://www.ncbi.nlm.nih.gov/pmc/articles/PMC2904283/

145. Macgillavry HD, Kerr JM, Kassner J, Frost NA, Blanpied TA. Shank-cortactin interactions control actin dynamics to maintain flexibility of neuronal spines and synapses. Eur J Neurosci. 2015;

146. McWilliams RR, Gidey E, Fouassier L, Weed S a, Doctor RB. Characterization of an ankyrin repeatcontaining Shank2 isoform (Shank2E) in liver epithelial cells. Biochem J [Internet]. 2004;380(Pt 1):18191. Available from: http://www.pubmedcentral.nih.gov/articlerender.fcgi?artid=1224161\&tool=pmcentrez\&rendertype=abstra ct

147. Du Y, Weed SA, Xiong W-C, Marshall TD, Parsons JT. Identification of a Novel Cortactin SH3 DomainBinding Protein and Its Localization to Growth Cones of Cultured Neurons. Mol Cell Biol [Internet]. 1998 Oct [cited 2020 Jul 7];18(10):5838-51. Available from: http://www.ncbi.nlm.nih.gov/pubmed/9742101 
148. A. Arnold, T. Motokura, T. Bloom, H. Kronenberg, J. Ruderman, H. Juppner HGK. The Putative Oncogene PRAD1 Encodes a Novel Cyclin. Cold Spring Harb Symp Quant Biol. 1991;56:93-7.

149. Hsi ED, Zukerberg LR, Yang WI, Arnold A. Cyclin D1/PRAD1 expression in parathyroid adenomas: an immunohistochemical study. J Clin Endocrinol Metab [Internet]. 1996 [cited 2020 Jul 3];81(5):1736-9. Available from: https://pubmed.ncbi.nlm.nih.gov/8626826/

150. Rosenberg CL, Wong E, Petty EM, Bale AE, Tsujimoto Y, Harris NL, et al. PRAD1, a candidate BCL1 oncogene: mapping and expression in centrocytic lymphoma. Proc Natl Acad Sci U S A [Internet]. 1991;88(21):9638-42. Available from:

http://www.pubmedcentral.nih.gov/articlerender.fcgi?artid=52773\&tool=pmcentrez\&rendertype=abstract

151. Rosenthal ET, Hunt T, Ruderman J V. Selective translation of mRNA controls the pattern of protein synthesis during early development of the surf clam, Spisula solidissima. Cell [Internet]. 1980 [cited 2020 Jul 3];20(2):487-94. Available from: https://pubmed.ncbi.nlm.nih.gov/7190072/

152. Motokura T, Bloom T, Kim HG, Jüppner H, Ruderman J V, Kronenberg HM, et al. A novel cyclin encoded by a bcl1-linked candidate oncogene. Nature [Internet]. 1991 Apr 11 [cited 2016 Mar 27];350(6318):512-5. Available from:

http://www.nature.com.www.libproxy.wvu.edu/nature/journal/v350/n6318/abs/350512a0.html

153. Xiong Y, Connolly T, Futcher B, Beach D. Human D-type cyclin. Cell [Internet]. 1991 [cited 2020 Jul 3];65(4):691-9. Available from: https://pubmed.ncbi.nlm.nih.gov/1827756/

154. Klein E a, Assoian RK. Transcriptional regulation of the cyclin D1 gene at a glance. J Cell Sci. 2008;121(Pt 23):3853-7.

155. Lavoie H, Gagnon J, Therrien M. ERK signalling: a master regulator of cell behaviour, life and fate. Nat Rev Mol Cell Biol [Internet]. 2020 Jun 23;(514). Available from: http://dx.doi.org/10.1038/s41580-0200255-7

156. Deshpande AAJA, Pastore A, Deshpande AAJA, Zimmermann Y, Hutter G, Weinkauf M, et al. 3'UTR mediated regulation of the cyclin D1 proto-oncogene. Cell Cycle [Internet]. 2009 [cited 2020 Jul 
20];8(21):3584-92. Available from: https://pubmed.ncbi.nlm.nih.gov/19823025/

157. Lolli G, Johnson LN. CAK-Cyclin-Dependent Activating Kinase: A key kinase in cell cycle control and a target for Drugs? Cell Cycle. 2005;4(4):565-70.

158. Musgrove EA, Caldon CE, Barraclough J, Stone A, Sutherland RL. Cyclin D as a therapeutic target in cancer. Nat Rev Cancer [Internet]. 2011 Aug [cited 2014 Nov 26];11(8):558-72. Available from: http://dx.doi.org/10.1038/nrc3090

159. Malumbres M, Barbacid M. Cell cycle, CDKs and cancer: A changing paradigm [Internet]. Vol. 9, Nature Reviews Cancer. Nature Publishing Group; 2009 [cited 2020 Jul 3]. p. 153-66. Available from: http://www.nature.com/articles/nrc2602

160. DeGregori J, Kowalik T, Nevins JR. Cellular targets for activation by the E2F1 transcription factor include DNA synthesis- and G1/S-regulatory genes. Mol Cell Biol [Internet]. 1995 [cited 2020 Jul 3];15(8):4215-24. Available from: https://www.ncbi.nlm.nih.gov/pmc/articles/PMC230660/

161. Santarius T, Shipley J, Brewer D, Stratton MR, Cooper CS. A census of amplified and overexpressed human cancer genes [Internet]. Vol. 10, Nature Reviews Cancer. Nature Publishing Group; 2010 [cited 2020 Jul 3]. p. 59-64. Available from: http://www.nature.com/articles/nrc2771

162. Jares P, Colomer D, Campo E. Genetic and molecular pathogenesis of mantle cell lymphoma: Perspectives for new targeted therapeutics [Internet]. Vol. 7, Nature Reviews Cancer. Nat Rev Cancer; 2007 [cited 2020 Jul 3]. p. 750-62. Available from: https://pubmed.ncbi.nlm.nih.gov/17891190/

163. Shan J, Zhao W, Gu W. Suppression of Cancer Cell Growth by Promoting Cyclin D1 Degradation. Mol Cell [Internet]. 2009;36(3):469-76. Available from: http://dx.doi.org/10.1016/j.molcel.2009.10.018

164. Arato-Ohshima T, Sawa H. Over-expression of cyclin D1 induces glioma invasion by increasing matrix metalloproteinase activity and cell motility. Int J Cancer [Internet]. 1999 Oct 29;83(3):387-92. Available from: http://doi.wiley.com/10.1002/\%28SICl\%291097-

0215\%2819991029\%2983\%3A3\%3C387\%3A\%3AAID-IJC15\%3E3.0.CO\%3B2-O

165. Oliver G. Opitz, Yasir Sulima, William C. Hahn, Hideki Harada, Hubert E. Blum AKR. Cyclin D1 
overexpression and p53 inactivation immortalize primary oral keratinocytes by a telomeraseindependent mechanism. J Clin Investig. 2001;108(5):665-7.

166. Yasui M, Yamamoto H, Ngan CY, Damdinsuren B, Sugita Y, Fukunaga H, et al. Antisense to cyclin D1 inhibits vascular endothelial growth factor-stimulated growth of vascular endothelial cells: implication of tumor vascularization. Clin Cancer Res [Internet]. 2006;12(15):4720-9. Available from: http://www.ncbi.nlm.nih.gov/pubmed/16899623

167. Roué G, Pichereau V, Lincet H, Colomer D, Sola B. Cyclin D1 mediates resistance to apoptosis through upregulation of molecular chaperones and consequent redistribution of cell death regulators. Oncogene [Internet]. 2008;27(2008):4909-20. Available from:

http://www.nature.com/doifinder/10.1038/onc.2008.126

168. Billard-Sandu C, Tao YG, Sablin MP, Dumitrescu G, Billard D, Deutsch E. CDK4/6 inhibitors in P16/HPV16-negative squamous cell carcinoma of the head and neck [Internet]. Vol. 277, European Archives of Oto-Rhino-Laryngology. Eur Arch Otorhinolaryngol; 2020 [cited 2020 Jul 3]. p. 1273-80. Available from: http://www.ncbi.nlm.nih.gov/pubmed/32162057

169. Roussel MF. The INK4 family of cell cycle inhibitors in cancer [Internet]. Vol. 18, Oncogene. Oncogene; 1999 [cited 2020 Jul 3]. p. 5311-7. Available from: http://www.ncbi.nlm.nih.gov/pubmed/10498883

170. Shah M, Nunes MR, Stearns V. CDK4/6 inhibitors: Game changers in the management of hormone receptor- positive advanced breast cancer? Oncol (United States) [Internet]. 2018 [cited 2020 Jul 3];32(5):216-22. Available from: http://www.ncbi.nlm.nih.gov/pubmed/29847850

171. Lim JSJ, Turner NC, Yap TA. CDK4/6 Inhibitors: Promising Opportunities beyond Breast Cancer. Cancer Discov [Internet]. 2016;6(7):697-9. Available from: http://cancerdiscovery.aacrjournals.org/cgi/doi/10.1158/2159-8290.CD-16-0563

172. Patnaik A, Rosen LS, Tolaney SM, Tolcher AW, Goldman JW, Gandhi L, et al. Efficacy and Safety of Abemaciclib, an Inhibitor of CDK4 and CDK6, for Patients with Breast Cancer, Non-Small Cell Lung Cancer, and Other Solid Tumors. Cancer Discov [Internet]. 2016;(July):740-54. Available from: 
http://cancerdiscovery.aacrjournals.org/content/early/2016/05/18/2159-8290.CD-16-0095.abstract

173. Kapranov P, Churchill G a, Holland MJ, Draghici S, Khatri P, Eklund a C, et al. RNA maps reveal new RNA classes and a possible function for pervasive transcription. Science (80- ). 2007;316(June):14848.

174. Comings DE. The Structure and Function of Chromatin. In: Advances in human genetics [Internet]. Adv Hum Genet; 1972 [cited 2020 Jul 7]. p. 237-431. Available from:

http://www.ncbi.nlm.nih.gov/pubmed/4578264

175. Thomas CA. The Genetic Organization of Chromosomes. Annu Rev Genet [Internet]. 1971 Dec 28 [cited 2020 Jul 7];5(1):237-56. Available from:

http://www.annualreviews.org/doi/10.1146/annurev.ge.05.120171.001321

176. Brosius J, Raabe CA. What is an RNA? A top layer for RNA classification. RNA Biol [Internet]. 2016 [cited 2020 Jul 7];13(2):140-4. Available from: https://www.ncbi.nlm.nih.gov/pmc/articles/PMC4829331/

177. Feinbaum R, Ambros V, Lee R. The C. elegans Heterochronic Gene lin-4 Encodes Small RNAs with Antisense Complementarity to lin-14. Cell. 2004;116(116):843-54.

178. Wightman B, Bürglin TR, Gatto J, Arasu P, Ruvkun G. Negative regulatory sequences in the lin-14 3'untranslated region are necessary to generate a temporal switch during Caenorhabditis elegans development. Genes Dev [Internet]. 1991 Oct 1 [cited 2020 Jul 7];5(10):1813-24. Available from: http://www.ncbi.nlm.nih.gov/pubmed/1916264

179. Pasquinelli AE, Reinhart BJ, Slack F, Martindale MQ, Kuroda MI, Maller B, et al. Conservation of the sequence and temporal expression of let-7 heterochronic regulatory RNA. Nature [Internet]. 2000 Nov [cited 2020 Jul 7];408(6808):86-9. Available from: http://www.nature.com/articles/35040556

180. Lee RC, Ambros V. An extensive class of small RNAs in Caenorhabditis elegans. Science (80- ) [Internet]. 2001 Oct 26 [cited 2020 Jul 7];294(5543):862-4. Available from:

http://www.ncbi.nlm.nih.gov/pubmed/11679672

181. Lau NC, Lim LP, Weinstein EG, Bartel DP. An abundant class of tiny RNAs with probable regulatory 
roles in Caenorhabditis elegans. Science (80- ) [Internet]. 2001 Oct 26 [cited 2020 Jul 7];294(5543):858-62. Available from: http://www.ncbi.nlm.nih.gov/pubmed/11679671

182. M L-Q, R R, W L, T T, Lagos-Quintana M, Rauhut $R$, et al. Identification of novel genes coding for small expressed RNAs. Science (80- ) [Internet]. 2001 [cited 2020 Jul 7];294(5543):853-8. Available from: https://pubmed.ncbi.nlm.nih.gov/11679670/

183. Kozomara A, Griffiths-Jones S. MiRBase: Integrating microRNA annotation and deep-sequencing data. Nucleic Acids Res [Internet]. 2011 Jan 1 [cited 2020 Jul 7];39(SUPPL. 1):D152-7. Available from: https://academic.oup.com/nar/article-lookup/doi/10.1093/nar/gkq1027

184. Griffiths-Jones S. The microRNA Registry. Nucleic Acids Res [Internet]. 2004 Jan 1 [cited 2020 Jul 7];32(90001):109D - 111. Available from: http://www.ncbi.nlm.nih.gov/pubmed/14681370

185. Ha M, Kim VN. Regulation of microRNA biogenesis [Internet]. Vol. 15, Nature Reviews Molecular Cell Biology. Nat Rev Mol Cell Biol; 2014 [cited 2020 Jul 7]. p. 509-24. Available from: https://pubmed.ncbi.nlm.nih.gov/25027649/

186. Denli AM, Tops BBJ, Plasterk RHA, Ketting RF, Hannon GJ. Processing of primary microRNAs by the Microprocessor complex. Nature [Internet]. 2004 [cited 2020 Jul 7];432(7014):231-5. Available from: https://pubmed.ncbi.nlm.nih.gov/15531879/

187. Okada C, Yamashita E, Lee SJ, Shibata S, Katahira J, Nakagawa A, et al. A high-Resolution structure of the pre-microrna nuclear export machinery. Science (80- ) [Internet]. 2009 [cited 2020 Jul 7];326(5957):1275-9. Available from: https://pubmed.ncbi.nlm.nih.gov/19965479/

188. Yoda M, Kawamata T, Paroo Z, Ye X, Iwasaki S, Liu Q, et al. ATP-dependent human RISC assembly pathways. Nat Struct Mol Biol [Internet]. 2010 [cited 2020 Jul 7];17(1):17-24. Available from: https://pubmed.ncbi.nlm.nih.gov/19966796/

189. Kawamata T, Tomari Y. Making RISC [Internet]. Vol. 35, Trends in Biochemical Sciences. Trends Biochem Sci; 2010 [cited 2020 Jul 7]. p. 368-76. Available from: https://pubmed.ncbi.nlm.nih.gov/20395147/ 
190. Jo MH, Shin S, Jung SR, Kim E, Song JJ, Hohng S. Human Argonaute 2 Has Diverse Reaction Pathways on Target RNAs. Mol Cell [Internet]. 2015 [cited 2020 Jul 7];59(1):117-24. Available from: https://pubmed.ncbi.nlm.nih.gov/26140367/

191. Jonas S, Izaurralde E. Towards a molecular understanding of microRNA-mediated gene silencing [Internet]. Vol. 16, Nature Reviews Genetics. Nat Rev Genet; 2015 [cited 2020 Jul 7]. p. 421-33. Available from: https://pubmed.ncbi.nlm.nih.gov/26077373/

192. Behm-Ansmant I, Rehwinkel J, Doerks T, Stark A, Bork P, Izaurralde E. mRNA degradation by miRNAs and GW182 requires both CCR4:NOT deadenylase and DCP1:DCP2 decapping complexes. Genes Dev [Internet]. 2006 Jul 15 [cited 2020 Jul 7];20(14):1885-98. Available from: http://www.ncbi.nlm.nih.gov/pubmed/16815998

193. Braun JE, Truffault V, Boland A, Huntzinger E, Chang C Te, Haas G, et al. A direct interaction between DCP1 and XRN1 couples mRNA decapping to 5' exonucleolytic degradation. Nat Struct Mol Biol [Internet]. 2012 Dec [cited 2020 Jul 7];19(12):1324-31. Available from: http://www.ncbi.nlm.nih.gov/pubmed/23142987

194. Valencia-Sanchez MA, Liu J, Hannon GJ, Parker R. Control of translation and mRNA degradation by miRNAs and siRNAs. Genes Dev [Internet]. 2006 Mar 1 [cited 2014 Jul 14];20(5):515-24. Available from: http://www.ncbi.nlm.nih.gov/pubmed/16510870

195. Brennecke J, Hipfner DR, Stark A, Russell RB, Cohen SM. bantam encodes a developmentally regulated microRNA that controls cell proliferation and regulates the proapoptotic gene hid in Drosophila. Cell [Internet]. 2003 Apr 4 [cited 2020 Jul 7];113(1):25-36. Available from: https://www.cell.com/cell/fulltext/S0092-8674(03)002319?_returnURL=https\%3A\%2F\%2Flinkinghub.elsevier.com\%2Fretrieve\%2Fpii\%2FS0092867403002319 \%3Fshowall\%3Dtrue

196. Llave C, Xie Z, Kasschau KD, Carrington JC. Cleavage of Scarecrow-like mRNA targets directed by a class of Arabidopsis miRNA. Science (80- ) [Internet]. 2002 Mar 26 [cited 2020 Jul 7];297(5589):2053-6. Available from: 
https://science.sciencemag.org/content/303/5666/2022.abstract?ijkey=fa077eaf0d0bf59d075a00b7ef41c 4f46dae7ba0\&keytype2=tf_ipsecsha\%3FlinkType\%3DABST\&journalCode=sci\&resid=303/5666/2022

197. Poy MN, Eliasson L, Krutzfeldt J, Kuwajima S, Ma X, MacDonald PE, et al. A pancreatic islet-specific microRNA regulates insulin secretion. Nature [Internet]. 2004 Nov [cited 2020 Jul 7];432(7014):226-30. Available from: http://www.nature.com/articles/nature03076

198. Cimmino A, Calin GA, Fabbri M, lorio M V, Ferracin M, Shimizu M, et al. miR-15 and miR-16 induce apoptosis by targeting BCL2. Proc Natl Acad Sci U S A [Internet]. 2005 Sep 27 [cited 2020 Jul 7];102(39):13944-9. Available from: http://www.ncbi.nlm.nih.gov/pubmed/16166262

199. Wang J, Liu X, Wu H, Ni P, Gu Z, Qiao Y, et al. CREB up-regulates long non-coding RNA, HULC expression through interaction with microRNA-372 in liver cancer. Nucleic Acids Res [Internet]. 2010 Sep [cited 2020 Jul 7];38(16):5366-83. Available from: http://www.ncbi.nlm.nih.gov/pubmed/20423907

200. Milligan MJ, Lipovich L. Pseudogene-derived IncRNAs: Emerging regulators of gene expression [Internet]. Vol. 6, Frontiers in Genetics. Frontiers Media SA; 2015 [cited 2020 Jul 7]. Available from: https://www.ncbi.nlm.nih.gov/pmc/articles/PMC4316772/

201. Brannan Cl, Dees EC, Ingram RS, Tilghman SM. The product of the H19 gene may function as an RNA. Mol Cell Biol [Internet]. 1990 Jan [cited 2020 Jul 7];10(1):28-36. Available from: http://www.ncbi.nlm.nih.gov/pubmed/1688465

202. Brockdorff N, Ashworth A, Kay GF, McCabe VM, Norris DP, Cooper PJ, et al. The product of the mouse Xist gene is a $15 \mathrm{~kb}$ inactive $\mathrm{X}$-specific transcript containing no conserved ORF and located in the nucleus. Cell. 1992;71(3):515-26.

203. Guttman M, Amit I, Garber M, French C, Lin MF, Feldser D, et al. Chromatin signature reveals over a thousand highly conserved large non-coding RNAs in mammals. Nature [Internet]. 2009 Mar 12 [cited 2020 Jul 7];458(7235):223-7. Available from: http://www.ncbi.nlm.nih.gov/pubmed/19182780

204. Katayama S, Tomaru Y, Kasukawa T, Waki K, Nakanishi M, Nakamura M, et al. Molecular biology: Antisense transcription in the mammalian transcriptome. Science (80- ) [Internet]. 2005 Sep 2 [cited 
2020 Jul 7];309(5740):1564-6. Available from:

https://science.sciencemag.org/content/309/5740/1564.long

205. Louro R, Smirnova AS, Verjovski-Almeida S. Long intronic noncoding RNA transcription: Expression noise or expression choice? [Internet]. Vol. 93, Genomics. Academic Press; 2009 [cited 2020 Jul 7]. p. 291-8. Available from:

https://www.sciencedirect.com/science/article/pii/S0888754308003042?via\%3Dihub

206. Seila AC, Calabrese JM, Levine SS, Yeo GW, Rahl PB, Flynn RA, et al. Divergent transcription from active promoters. Science (80- ) [Internet]. 2008 Dec 19 [cited 2020 Jul 7];322(5909):1849-51. Available from: http://www.ncbi.nlm.nih.gov/pubmed/19056940

207. Rinn JL, Kertesz M, Wang JK, Squazzo SL, Xu X, Brugmann SA, et al. Functional Demarcation of Active and Silent Chromatin Domains in Human HOX Loci by Noncoding RNAs. Cell [Internet]. 2007 [cited 2020 Jul 7];129(7):1311-23. Available from: https://pubmed.ncbi.nlm.nih.gov/17604720/

208. Kung JTY, Colognori D, Lee JT. Long noncoding RNAs: Past, present, and future [Internet]. Vol. 193, Genetics. Genetics Society of America; 2013 [cited 2020 Jul 7]. p. 651-69. Available from: https://www.ncbi.nlm.nih.gov/pmc/articles/PMC3583990/\#bib131

209. Hung T, Wang Y, Lin MF, Koegel AK, Kotake Y, Grant GD, et al. Extensive and coordinated transcription of noncoding RNAs within cell cycle promoters. Nat Genet [Internet]. 2011 [cited 2020 Jul 7];43(7):621. Available from: https://www.ncbi.nIm.nih.gov/pmc/articles/PMC3652667/

210. Martianov I, Ramadass A, Serra Barros A, Chow N, Akoulitchev A. Repression of the human dihydrofolate reductase gene by a non-coding interfering transcript. Nature [Internet]. 2007 Feb 8 [cited 2020 Jul 7];445(7128):666-70. Available from: http://www.ncbi.nlm.nih.gov/pubmed/17237763

211. Avissar M, McClean MD, Kelsey KT, Marsit CJ. MicroRNA expression in head and neck cancer associates with alcohol consumption and survival. Carcinogenesis [Internet]. 2009 Dec;30(12):2059-63. Available from: https://academic.oup.com/carcin/article-lookup/doi/10.1093/carcin/bgp277

212. Darido C, Georgy SR, Wilanowski T, Dworkin S, Auden A, Zhao Q, et al. Targeting of the tumor 
suppressor GRHL3 by a miR-21-dependent proto-oncogenic network results in PTEN loss and tumorigenesis. Cancer Cell [Internet]. 2011 Nov 15;20(5):635-48. Available from: http://www.ncbi.nlm.nih.gov/pubmed/22094257

213. Hsu C-M, Lin P-M, Wang Y-M, Chen Z-J, Lin S-F, Yang M-Y. Circulating miRNA is a novel marker for head and neck squamous cell carcinoma. Tumour Biol [Internet]. 2012 Dec;33(6):1933-42. Available from: http://www.ncbi.nlm.nih.gov/pubmed/22811001

214. Liu C-J, Kao S-Y, Tu H-F, Tsai M-M, Chang K-W, Lin S-C. Increase of microRNA miR-31 level in plasma could be a potential marker of oral cancer. Oral Dis [Internet]. 2010 May;16(4):360-4. Available from: http://www.ncbi.nlm.nih.gov/pubmed/20233326

215. Liu C-J, Lin S-C, Yang C-C, Cheng H-W, Chang K-W. Exploiting salivary miR-31 as a clinical biomarker of oral squamous cell carcinoma. Head Neck [Internet]. 2012 Feb;34(2):219-24. Available from: http://www.ncbi.nlm.nih.gov/pubmed/22083872

216. Bourguignon LYW, Earle C, Wong G, Spevak CC, Krueger K. Stem cell marker (Nanog) and Stat-3 signaling promote MicroRNA-21 expression and chemoresistance in hyaluronan/CD44-activated head and neck squamous cell carcinoma cells. Oncogene [Internet]. 2012 Jan 20;31(2):149-60. Available from: http://www.nature.com/articles/onc2011222

217. Yu Y, Kanwar SS, Patel BB, Oh P-S, Nautiyal J, Sarkar FH, et al. MicroRNA-21 induces stemness by downregulating transforming growth factor beta receptor 2 (TGF $\beta 2$ ) in colon cancer cells. Carcinogenesis [Internet]. 2012 Jan;33(1):68-76. Available from: http://www.ncbi.nlm.nih.gov/pubmed/22072622

218. Liu Z-L, Wang H, Liu J, Wang Z-X. MicroRNA-21 (miR-21) expression promotes growth, metastasis, and chemo- or radioresistance in non-small cell lung cancer cells by targeting PTEN. Mol Cell Biochem [Internet]. 2013 Jan;372(1-2):35-45. Available from: http://www.ncbi.nlm.nih.gov/pubmed/22956424

219. Reis PP, Tomenson M, Cervigne NK, Machado J, Jurisica I, Pintilie M, et al. Programmed cell death 4 loss increases tumor cell invasion and is regulated by miR-21 in oral squamous cell carcinoma. Mol 
Cancer [Internet]. 2010 Sep 10;9:238. Available from: http://www.ncbi.nlm.nih.gov/pubmed/20831814

220. Liu C-J, Tsai M-M, Hung P-S, Kao S-Y, Liu T-Y, Wu K-J, et al. miR-31 ablates expression of the HIF regulatory factor FIH to activate the HIF pathway in head and neck carcinoma. Cancer Res [Internet]. 2010 Feb 15;70(4):1635-44. Available from: http://www.ncbi.nlm.nih.gov/pubmed/20145132

221. Lajer CB, Nielsen FC, Friis-Hansen L, Norrild B, Borup R, Garnæs E, et al. Different miRNA signatures of oral and pharyngeal squamous cell carcinomas: a prospective translational study. $\mathrm{Br} \mathrm{J}$ Cancer [Internet]. 2011 Mar 15;104(5):830-40. Available from: http://www.nature.com/articles/bjc201129

222. Jakymiw A, Patel RS, Deming N, Bhattacharyya I, Shah P, Lamont RJ, et al. Overexpression of dicer as a result of reduced let-7 MicroRNA levels contributes to increased cell proliferation of oral cancer cells. Genes, Chromosom Cancer [Internet]. 2010 Jun;49(6):549-59. Available from: http://doi.wiley.com/10.1002/gcc.20765

223. Yang W-H, Lan H-Y, Huang C-H, Tai S-K, Tzeng C-H, Kao S-Y, et al. RAC1 activation mediates Twist1induced cancer cell migration. Nat Cell Biol [Internet]. 2012 Mar 11;14(4):366-74. Available from: http://www.ncbi.nlm.nih.gov/pubmed/22407364

224. Chen Z, Jin Y, Yu D, Wang A, Mahjabeen I, Wang C, et al. Down-regulation of the microRNA-99 family members in head and neck squamous cell carcinoma. Oral Oncol [Internet]. 2012 Aug;48(8):686-91. Available from: http://www.ncbi.nlm.nih.gov/pubmed/22425712

225. Uesugi A, Kozaki K-I, Tsuruta T, Furuta M, Morita K-I, Imoto I, et al. The tumor suppressive microRNA miR-218 targets the mTOR component Rictor and inhibits AKT phosphorylation in oral cancer. Cancer Res [Internet]. 2011 Sep 1;71(17):5765-78. Available from:

http://www.ncbi.nlm.nih.gov/pubmed/21795477

226. Ghafouri-Fard S, Mohammad-Rahimi H, Jazaeri M, Taheri M. Expression and function of long noncoding RNAs in head and neck squamous cell carcinoma. Exp Mol Pathol [Internet]. 2020;112(October 2019):104353. Available from: https://doi.org/10.1016/j.yexmp.2019.104353

227. Han X, Xu Z, Tian G, Tang Z, Gao J, Wei Y, et al. Suppression of the long non-coding RNA MALAT-1 
impairs the growth and migration of human tongue squamous cell carcinoma SCC4 cells. Arch Med Sci [Internet]. 2019;15(4):992-1000. Available from: https://www.termedia.pl/doi/10.5114/aoms.2018.73343

228. Fang Z, Zhang S, Wang Y, Shen S, Wang F, Hao Y, et al. Long non-coding RNA MALAT-1 modulates metastatic potential of tongue squamous cell carcinomas partially through the regulation of small proline rich proteins. BMC Cancer [Internet]. 2016 Dec 1;16(1):706. Available from:

http://bmccancer.biomedcentral.com/articles/10.1186/s12885-016-2735-x

229. Salmena L, Poliseno L, Tay Y, Kats L, Pandolfi PP. A ceRNA hypothesis: the Rosetta Stone of a hidden RNA language? Cell [Internet]. 2011 Aug 5 [cited 2014 Jul 9];146(3):353-8. Available from: http://www.cell.com/article/S0092867411008129/fulltext

230. Yang C, Wu D, Gao L, Liu X, Jin Y, Wang D, et al. Competing endogenous RNA networks in human cancer: hypothesis, validation, and perspectives [Internet]. Vol. 5, Oncotarget. Impact Journals; 2016 [cited 2016 Mar 16]. Available from:

http://www.impactjournals.com/oncotarget/index.php?journal=oncotarget\&page=article\&op=view\&path\% $5 B \% 5 D=7266 \&$ path\%5B\%5D=20762

231. Kartha R V., Subramanian S. Competing endogenous RNAs (ceRNAs): new entrants to the intricacies of gene regulation. Front Genet [Internet]. 2014 Jan [cited 2014 Jul 21];5:8. Available from: http://www.pubmedcentral.nih.gov/articlerender.fcgi?artid=3906566\&tool=pmcentrez\&rendertype=abstra ct

232. Tay Y, Rinn J, Pandolfi PP. The multilayered complexity of ceRNA crosstalk and competition. Nature [Internet]. 2014 Jan 15 [cited 2016 Aug 31];505(7483):344-52. Available from: http://www.nature.com/doifinder/10.1038/nature12986

233. Tay $Y$, Kats L, Salmena L, Weiss D, Tan SM, Ala U, et al. Coding-independent regulation of the tumor suppressor PTEN by competing endogenous mRNAs. Cell [Internet]. 2011;147(2):344-57. Available from: http://dx.doi.org/10.1016/j.cell.2011.09.029

234. Karreth FA, Reschke M, Ruocco A, Ng C, Chapuy B, Léopold V, et al. The BRAF pseudogene functions 
as a competitive endogenous RNA and induces lymphoma in vivo. Cell. 2015;161(2):319-32.

235. Denzler R, Agarwal V, Stefano J, Bartel DP, Stoffel M. Assessing the ceRNA Hypothesis with Quantitative Measurements of miRNA and Target Abundance. Mol Cell [Internet]. 2014 Jun 5 [cited 2020 Jul 7];54(5):766-76. Available from: http://www.ncbi.nlm.nih.gov/pubmed/24793693

236. Wang K, Liu F, Zhou LY, Long B, Yuan SM, Wang Y, et al. The long noncoding RNA CHRF regulates cardiac hypertrophy by targeting miR-489. Circ Res [Internet]. 2014 Apr 25 [cited 2020 Jul 7];114(9):1377-88. Available from: https://www.ahajournals.org/doi/10.1161/CIRCRESAHA.114.302476

237. Wang K, Liu CY, Zhou LY, Wang JX, Wang M, Zhao B, et al. APF IncRNA regulates autophagy and myocardial infarction by targeting miR-188-3p. Nat Commun [Internet]. 2015 Nov 10 [cited 2020 Jul 7];6(1):6779. Available from: http://www.nature.com/articles/ncomms7779

238. Zhang Y, Ke X, Liu J, Ma X, Liu Y, Liang D, et al. Characterization of circRNA-associated ceRNA networks in patients with nonvalvular persistent atrial fibrillation. Mol Med Rep [Internet]. 2019 Nov 26 [cited 2020 Jul 7];19(1):638-50. Available from: http://www.spandidospublications.com/10.3892/mmr.2018.9695

239. Zhou F, Xie S, Li J, Duan S. Long noncoding RNA HOTAIR promotes cell apoptosis by sponging miR221 in Parkinson's disease. RSC Adv [Internet]. 2019 Sep 18 [cited 2020 Jul 7];9(51):29502-10. Available from: http://xlink.rsc.org/?DOI=C9RA06107J

240. Wang LK, Chen XF, He DD, Li Y, Fu J. Dissection of functional IncRNAs in Alzheimer's disease by construction and analysis of IncRNA-mRNA networks based on competitive endogenous RNAs. Biochem Biophys Res Commun [Internet]. 2017 Apr 8 [cited 2020 Jul 7];485(3):569-76. Available from: https://www.sciencedirect.com/science/article/pii/S0006291X16320174?casa_token=yUUIPzybpMoAAA AA:MmU6wTrn9P6Xm8LPxodrb0awC_E0OzhqArDryzeaAyEaugzD43q2w5xYQExc3iu1aN8v4t6G31U

241. Liu X hua, Sun M, Nie F qi, Ge Y bin, Zhang E bao, Yin D dan, et al. Lnc RNA HOTAIR functions as a competing endogenous RNA to regulate HER2 expression by sponging miR-331-3p in gastric cancer. Mol Cancer [Internet]. 2014 Apr 28 [cited 2020 Jul 7];13(1):92. Available from: http://molecular- 
cancer.biomedcentral.com/articles/10.1186/1476-4598-13-92

242. Bai M, Yuan M, Liao H, Chen J, Xie B, Yan D, et al. OCT4 pseudogene 5 upregulates OCT4 expression to promote proliferation by competing with miR-145 in endometrial carcinoma. Oncol Rep [Internet]. 2015 Apr 1 [cited 2020 Jul 7];33(4):1745-52. Available from: https://www.spandidospublications.com/10.3892/or.2015.3763

243. Tian W, Jiang C, Huang Z, Xu D, Zheng S. Comprehensive analysis of dysregulated IncRNAs, miRNAs and mRNAs with associated ceRNA network in esophageal squamous cell carcinoma. Gene [Internet]. 2019 May 15 [cited 2020 Jul 7];696:206-18. Available from:

https://www.sciencedirect.com/science/article/abs/pii/S0378111919301830?via\%3Dihub

244. Wang X, Hu K Bin, Zhang YQ, Yang CJ, Yao HH. Comprehensive analysis of aberrantly expressed profiles of IncRNAs, miRNAs and mRNAs with associated ceRNA network in cholangiocarcinoma. Cancer Biomarkers [Internet]. 2018 Dec 6 [cited 2020 Jul 7];23(4):549-59. Available from: https://www.medra.org/servlet/aliasResolver?alias=iospress\&doi=10.3233/CBM-181684

245. Wang H, Niu L, Jiang S, Zhai J, Wang P, Kong F, et al. Comprehensive analysis of aberrantly expressed profiles of IncRNAs and miRNAs with associated ceRNA network in muscleinvasive bladder cancer. Oncotarget [Internet]. 2016 [cited 2020 Jul 7];7(52):86174-85. Available from: https://pubmed.ncbi.nlm.nih.gov/27863388/ 


\section{Study 1: Disparate Survival of Late-stage Male Oropharyngeal \\ Cancer in Appalachia}

Brenen W. Papenberg ${ }^{1}$, Jessica L. Allen ${ }^{1}$, Steven M. Markwell ${ }^{1}$, Erik T. Interval ${ }^{2}$, Phillip A. Montague ${ }^{2}$, Christopher J. Johnson ${ }^{3}$ and Scott A. Weed ${ }^{1 *}$

${ }^{1}$ Department of Biochemistry, Program in Cancer Cell Biology, ${ }^{2}$ Department of Otolaryngology, Head and Neck Surgery, West Virginia University, Morgantown, West Virginia, 26506. ${ }^{3}$ Cancer Data Registry of Idaho, Boise, ID 83701

Running title. Outcome Disparity in Appalachian Male Oral Cancer

Keywords. Oral Cavity and Pharyngeal Cancer, Appalachia, Survival, Disparity, Tobacco, HPV, Subsites.

Financial support: NIH/NIGMS grants U54GM104942 and P20GM103434. Mr. Johnson was supported with Federal funds from the National Cancer Institute, NIH, HHS, under Contract No. HHSN261201800006I and the Centers for Disease Control and Prevention, HHS, under Cooperative Agreement 1NU58DP006270. The content is solely the responsibility of the authors and does not necessarily represent the official views of the $\mathrm{NIH}$ or the Centers for Disease Control and Prevention. This work was also supported by the West Virginia University Department of Biochemistry and Office of Research and Graduate Education.

Corresponding Author: "Scott A. Weed, West Virginia University Cancer Institute, P.O. Box 9300, Morgantown, WV 26506, Phone: 304-293-3016, Fax: 304-293-4667, scweed@hsc.wvu.edu

Published in Scientific Reports (2020) 


\section{Abstract}

The United States Appalachian region harbors a higher cancer burden than the rest of the nation, with disparate incidence of head and neck squamous cell carcinomas (HNSCC), including oral cavity and pharynx (OC/P) cancers. Whether elevated HNSCC incidence generates survival disparities within Appalachia is unknown. To address this, HNSCC survival data for 259,737 tumors from the North American Association for Central Cancer Registries (NAACCR) 2007-2013 cohort were evaluated, with age-adjusted relative survival (RS) calculated based on staging, race, sex, and Appalachian residence. Tobacco use, a primary HNSCC risk factor, was evaluated through the Behavioral Risk Factor Surveillance System (BRFSS) from Appalachian states. Decreased OC/P RS was found in stage IV Appalachian white males within a subset of states. The survival disparity was confined to human papillomavirus (HPV)-associated oropharyngeal cancers, specifically the oropharynx subsite. This correlated with significantly higher smoking and male smokeless tobacco use in most Appalachian disparity states. Lower survival of Appalachian males with advanced-stage HPV-associated oropharyngeal cancers suggests pervasive tobacco consumption likely generates more aggressive tumors at HPV-associated oropharynx subsites than national averages. Comprehensive tobacco and HPV status should therefore be evaluated prior to considering treatment de-intensification regimens for HPV-associated oropharyngeal cancers in populations with high tobacco consumption. 


\section{Introduction}

HNSCC involves the epithelium of the oral cavity, pharynx and larynx. OC/P cancers are a major HNSCC subset, with nearly 11,000 deaths predicted in the US in 2020[1]. Risk factors include tobacco and alcohol use, and highrisk human papillomavirus (HPV) infection[2,3]. OC/P cancers are subdivided into HPV-associated oropharynx (HPV-associated) and non-HPV-associated oral cavity, hypopharynx, and nasopharynx (non-HPV-associated) cancers[4]. These designations are supported by studies indicating that non-HPV-associated cancers are primarily tobacco/alcohol induced, whereas HPV-associated cancers are predominantly caused by HPV infection[5]. Furthermore, HPV-negative or non-HPV-associated cancers consistently have poorer outcomes than HPV-positive or HPV-associated cancers, segregating these cancers as distinct diseases with differential clinical management[6,7]. While national incidence of non-HPV-associated cancers is decreasing due to tobacco cessation, HPV-associated cancers are increasing due to rising infection rates[8]. Regarding race, blacks with HNSCC present with more advanced disease, are older and have worse survival than whites, denoting a racial disparity[9-11]. Increased screening for HPV coupled with subsite analysis indicates that blacks with HNSCC have less HPV-positive cancer than whites, explaining differences in survival[12,13]. Consistent with this, HPVassociated cancers continue to increase in white males and in rural areas $[4,14,15]$.

The Appalachian region encompasses 205,000 square miles across 420 counties in 13 contiguous states[16]. Forty-two percent of Appalachia is rural, with poverty rates in many counties 1.5 times above the national mean[16]. The region is over $80 \%$ white, with several states having higher percentages of oral and other HPVassociated cancers, and higher smoking and smokeless tobacco use[17-19]. Aggregate risk factors experienced by Appalachian residents contribute to a high allostatic load capable of generating cancer disparities in a region with low ethnic diversity[20]. Incidence disparities within Appalachia are well recognized[21] with rates higher for HPV- and tobacco-related malignancies[22-24]. Elevated risk factor exposure has been suggested as the prime reason for disparate incidence in Appalachian male HNSCC[24,25]. However, due to gaps in data availability and small patient numbers at the county level, details on how increased incidence impacts HNSCC survival in Appalachia have not been rigorously evaluated[25]. Here we have evaluated the RS of all HNSCC in the majority of Appalachian states from 2007-2013 and have identified an outcome disparity in white males with oropharynx cancers that corresponds with elevated smoking and smokeless tobacco use in the region. 


\section{Materials and Methods}

\section{HNSCC Cohort and Relative Survival Data}

All methods were carried out in accordance with relevant guidelines and regulations. Survival data were generated by NAACCR and provided in Surveillance, Epidemiology, and End Results (SEER) ${ }^{\star}$ Stat approved under NAACCR IRB protocol 16-14, where all patient data was obtained following informed consent. Patients were diagnosed from 2007-2013 with tumors of International Classification of Diseases for Oncology (ICD-O)-3 histology types 8050-8084, 8120-8131 (squamous and transitional epithelium). Selecting all primary tumors matching selection criteria, the cohort includes tumors of the oral cavity $(n=55,620 / 259,737,21.4 \%)$ including ICD-O-3 site groups of tongue (C02.0-02.3, 02.9), floor of mouth (C04.0-04.9), and gum and other mouth (C03.0-03.9, C05.0, C05.8-05.9, C06.0-06.9); oropharynx ( $n=81,170 / 259,737,31.3 \%)$ including ICD-O-3 site groups of tongue (C01.9, C02.4, C02.8), gum and other mouth (C05.1-05.2), tonsil (C09.0-09.9), oropharynx (C10.0-10.9), and "other oral cavity and pharynx" (C14.0-14.8); "other pharynx" ( $\mathrm{n}=17,731 / 259,737,6.8 \%)$ including ICD-O-3 site groups of nasopharynx (C11.0-11.9) and hypopharynx (C12.9-13.9); and larynx ( $n=60,296 / 259,737,23.2 \%)$ including ICD-O-3 site group larynx (C32.0-32.9). Remaining tumors are additional primaries of miscellaneous ICD-O-3 site groups ( $n=44,916 / 259,737,17.3 \%)$. The cohort includes variables denoting whether or not patients were Appalachian county residents at the time of diagnosis, and county economic status from the Appalachian Regional Commission (ARC)[26]. Appalachian states with survival data fit for use through the reporting period included New York, Pennsylvania, West Virginia, Kentucky, North Carolina, South Carolina, Georgia, and Alabama[27]. The majority of cases were white ( $n=221,939 / 259,737,85.4 \%)$, stage IV ( $n=84,253 / 259,737,32.4 \%)$, and male $(n=193,647 / 259,737$, 74.6\%). Cases were evaluated using the Ederer II method and the "U.S. by race (W, B, AIAN, API) and Canada 1995-2012, Ages 0-99, State-county (modeled by varied state-county-ses)" life table. Included cases were microscopically confirmed for malignant behavior and followed at monthly intervals. Excluded cases were those that were diagnosed solely via death certificate or autopsy, and alive cases 
with no survival time. Age-adjusted RS, defined as net survival measure representing cancer survival in the absence of other causes of death[28], was calculated using SEER*Stat for each patient stratification, with z-tests for significance testing between each group using an alpha level of 0.05 . Cumulative (C)RS was used to compare groups with five years of available RS data and the maximum available follow-up RS was used to compare groups that failed to reach five years of follow-up.

\section{Mapping}

Maps were generated using 2014 cartographic boundary shapefiles from the United States Census Bureau[29] and Quantum Geographic Information System (QGIS) 3.2.2 software[30]. Appalachian state-Appalachian county refers to a county within Appalachia. Appalachian state-non-Appalachian county refers to a county outside of the Appalachian region, but within a state containing Appalachian counties. Non-Appalachian states lack Appalachian counties.

\section{TCGA Cohort and Associated Clinical Data}

Data from The Cancer Genome Atlas (TCGA) were obtained from the Broad Genomic Data Analysis Center (GDAC) Firehose 2016_01_28 TCGA-HNSC cohort consisting of 529 patients with tumors of the oral cavity $(n=320 / 529,60.5 \%)$, pharynx $(n=92 / 529,17.4 \%)$, and larynx $(n=117 / 529,22.1 \%)$. Clinical data and patient characteristics were retrieved from the level 4 TCGA clinical data file All_CDEs.txt and cBioPortal[31,32]. OC/P patients were stratified by HPV-associated or non-HPV-associated subsite defined by the Centers for Disease Control (CDC)[33] and by confirmed HPV status. All patients were evaluated for HPV in the All_CDEs.txt file, denoted by variable "hpv_status". Kaplan-Meier P-values were calculated using Mantel-Cox log-rank test and were validated by an independent biostatistician.

\section{Appalachian Tobacco Use Data and Analysis}

Data for 2016 current smoker and smokeless tobacco user frequency, weighted frequency, prevalence, and confidence intervals for Appalachian and non-Appalachian counties were calculated and provided by BRFSS coordinators from Appalachian states. Current smoker status was calculated using CDC BRFSS Tobacco Use Question 1 and 2 from the 2016 questionnaire. Current smokeless status was calculated using CDC BRFSS Tobacco Use Question 3. Non-Appalachian state tobacco use data were acquired from CDC BRFSS[19]. 
Statistical significance was determined within states and between regions using G-tests for independence without Yates' correction using BRFSS frequency $(\mathrm{N})$ values and an alpha value of 0.05 . BRFSS recommends caution interpreting results with less than 50 respondents. Bonferroni correction was used where applicable to reduce type I error. Due to changes in sample composition and weighting methodology in 2011 , data from years after 2011 cannot be directly compared to previous years[34].

\section{Results}

\section{Identification of a Survival Disparity in White Appalachian Males with Stage IV Oral Cavity and}

\section{Pharyngeal Cancer}

The NAACCR cohort from $2007-2013$ in this study contained 145,823 OC/P tumors and 57,805 laryngeal tumors (Table 1, Fig. 1a, Fig. S1). The eight NAACCR-reporting Appalachian states cover $67.6 \%$ of all Appalachian counties and $72.3 \%$ of the Appalachian population[35]. The OC/P and laryngeal cohorts reflect overall Appalachian demographics, with a higher percentage of white cases and lower percentage of ethnic minorities than non-Appalachia (Table 1). Except for lower CRS in Appalachian OC/P and laryngeal cancers, all other parameters were nearly identical to national averages in each disease (Table 1). The majority of OC/P and laryngeal cancer cases came from distressed or transitional Appalachian counties.

RS was measured among OC/P and laryngeal cancer patients by Appalachian or non-Appalachian residency at time of diagnosis (Fig. 1a, Fig S1). When stratified by American Joint Committee on Cancer (AJCC)-6 stages I to IV, stage IV patients had the highest patient numbers and lowest CRS for each disease (OC/P; $n=63,396 / 116,595, C R S=45.5 \%$; laryngeal; $n=14,963 / 51,362 ; C R S=67.1 \%)$, and were selected for further evaluation (Fig. 1b and Fig. S1). OC/P and laryngeal patients were stratified by sex, race, and Appalachian residency (Fig. 1c, 1d; Fig S1). Survival analyses indicated that white Appalachian OC/P males ( $n=5,014 / 53,696$; $\mathrm{CRS}=44.9 \%$ ) displayed a significantly lower CRS compared to white non-Appalachian males (Fig. 1c; $\mathrm{n}=37,843 / 53,696 ; \mathrm{CRS}=49.2 \%), \mathrm{P}=0.00001$. In OC/P, there was no significant difference in CRS between white Appalachian females $(n=1,381 / 53,696 ; C R S=43.1 \%)$ and white non-Appalachian females $(n=9,458 / 53,696$; $\mathrm{CRS}=43.7 \%$ ) (Fig. 1c) or between black Appalachian males ( $\mathrm{n}=387 / 7,342 ; \mathrm{CRS}=35.8 \%$ ) and black nonAppalachian males ( $n=5,297 / 7,342 ; C R S=34.4 \%$ ) (Fig. 1d). Black Appalachian females $(n=116 / 7,342$; threeyear $\mathrm{RS}=46.1 \%$ ) failed to reach five-year follow-up for CRS comparison, but there was no observed significance 
between black non-Appalachian females at the latest available follow-up year ( $n=1,540 / 7,342$; three-year RS $=34.8 \%$ ) (Fig. 1d). No significant differences in CRS were observed for Appalachian laryngeal cancers between any sex or race (Fig. S1). White Appalachian males with stage IV OC/P were selected for further study due to their significantly different survival.

\section{The Survival Disparity in White Appalachian Male Stage IV OC/P is Specific to Appalachian Counties within Select Appalachian States}

To elucidate whether the observed stage IV white Appalachian male OC/P survival disparity is specific to Appalachian counties within Appalachian states, patients were stratified by Appalachian or non-Appalachian county and state residency. Appalachian states-Appalachian counties ( $n=5,014 / 42,857$; $C R S=44.9 \%)$ displayed

a significantly lower CRS compared to Appalachian states-non-Appalachian counties ( $n=9,700 / 42,857$; CRS $=47.9 \%$ ), $P=0.00017$ and non-Appalachian states ( $n=28,143 / 42,857 ; C R S=49.6 \%$ ), $P=0.00032$ (Fig. $2 a$ and b). This indicates that the survival disparity is specifically due to cancer survival within Appalachia, not to contributions from adjacent non-Appalachian counties within Appalachian state borders. There was no significant difference in CRS between non-Appalachian states and Appalachian states-non-Appalachian counties (Fig. 2a).

To determine if the stage IV white Appalachian male OC/P survival disparity could be identified at the state level, patients within Appalachian counties were stratified by state residency. Significantly lower CRS was observed for Appalachian counties in Alabama ( $n=626 / 31,423$; CRS=39.7\%), $P=0.0006$, Kentucky $(n=372 / 31,423$; CRS $=39.6 \%), P=0.0101$, Pennsylvania $(n=1,695 / 31,423 ; \quad C R S=45.3 \%), P=0.0047$, and West Virginia $(n=587 / 31,423 ; C R S=35.3 \%), P=0.007$ compared to non-Appalachian states $(n=28,143 / 31,423 ; C R S=49.6 \%)$

(Fig. 2c and d). All other analyzed Appalachian states did not have a significantly different CRS (Supplementary Figure S2).

The White Appalachian Male Stage IV OC/P Survival Disparity is Primarily Found in HPV-Associated Oropharyngeal Subsites

To determine if the stage IV white Appalachian male OC/P disparity is predominantly present within a specific $\mathrm{OC} / \mathrm{P}$ subregion, cases were initially divided into non-HPV-associated or HPV-associated cancer subtypes for survival analysis. HPV-associated cancers frequently contain oral mucosa with HPV DNA, and are defined as HPV-associated oropharynx by the CDC[33]. Remaining subsites are not associated with HPV infection and 
represent non-HPV-associated cancer (Fig. 3a). First, we evaluated the CDC definitions as predictors of HPV infection in HNSCC by using patients in the TCGA cohort, where all patients have known HPV status. Importantly, patients were evaluated for overall survival, since HPV is not a variable collected by NAACCR and thus could not be assessed in the Appalachian cohort. TCGA patients were additionally stratified as HPV-associated and non-HPV-associated using subsite information, then compared to outcomes based on confirmed HPV status. HPV-positive white male stage IV TCGA patients have an undefined median survival with five-year overall survival of $71 \%$, whereas HPV-negative patients have median survival of 47.0 months and five-year overall survival of 38\% (Fig. 3b). Similarly, HPV-associated cancer patients from the same cohort have median survival of 68.4 months and five-year overall survival of $61 \%$, while non-HPV-associated patients have lower median survival of 30.1 months and five-year overall survival of $37 \%$. While the survival trends regarding HPV status are in general agreement between the NAACCR and TCGA cohorts, the difference in five-year overall survival is greater when comparing HPV-positive (71\%) and HPV-associated (61\%) patients than between HPV-negative (38\%) and non-HPV-associated (37\%) patients. This suggests that the CDC non-HPV-associated subsite designation is a better predictor for HPV negativity than the HPV-associated subsite designation is for HPV positivity.

To elucidate the discordance between HPV-positive and HPV-associated patients in explaining the observed Appalachian survival differences, TCGA patients were separated into non-HPV-associated cancers and into the specific HPV-associated oropharynx subsites, with the HPV status determined for each grouping. Using this breakdown, non-HPV-associated cancers are mostly HPV-negative, whilst HPV-associated cancers contain a mixture of HPV-positive and HPV-negative cases (Fig. 3c). These findings potentially explain the discrepancy between HPV-positive and HPV-associated outcomes, and also point to confounding factors such as tobacco/alcohol use or socioeconomic status (SES) that may exist in patient cohorts stratified solely on the basis of HPV-association.

White male stage IV cancer cases in the NAACCR cohort were next stratified by HPV-association and Appalachian residency. Appalachian patients with HPV-associated cancers $(n=3,449 / 33,281 ; C R S=53.1 \%)$ displayed a significantly lower CRS compared to HPV-associated patients in non-Appalachian states $(n=20,399 / 33,281 ; C R S=57.7 \%), P=0.0118$ (Fig. 3d). No significant difference was found in CRS between nonHPV-associated patients in Appalachian counties $(n=1,577 / 33,281$; CRS $=29.5 \%)$ compared to non-HPV- 
associated patients in non-Appalachian states $(n=7,856 / 33,281 ; C R S=30.9 \%)$. These results suggest that the stage IV white Appalachian male OC/P survival disparity is driven by lower survival of HPV-associated oropharyngeal patients. Appalachian states with the overall stage IV white Appalachian male OC/P survival disparity identified in Figure $2 \mathrm{c}$ were evaluated separately and trended in a similar manner (Supplementary Figure 3).

White male stage IV patients in the NAACCR cohort were subsequently stratified by each HPV-associated oropharyngeal region to delineate the contributions of each subsite on Appalachian survival. A significant difference in survival was observed between patients with primary oropharynx tumors in Appalachia $(n=325 / 2,807 ; C R S=24.4 \%)$ and non-Appalachian counties $(n=649 / 2,807 ; C R S=36.9 \%), p=0.0399$ as well as non-Appalachian states ( $n=1,833 / 2,807 ; C R S=37.5 \%), P=0.0394$ (Fig. 3e). No significant differences were observed between patients with primary tonsil tumors in Appalachia ( $n=1,603 / 14,110$; CRS=55.8\%) and nonAppalachia states $(n=9,240 / 14,110 ; C R S=63.3 \%)$ or counties $(n=3,267 / 14,110 ; C R S=59.6 \%)$. Similarly, no significant differences were observed between patients with primary tongue tumors in Appalachia $(n=1,579 / 14,345 ; \quad C R S=54.8 \%)$ and non-Appalachian states $(n=9,625 / 14,345 ; \quad C R S=55.9 \%)$ or counties $(n=3,141 / 14,345 ; C R S=55.1 \%)$ (Fig. 3f, 3g). Other oropharyngeal subsites lacked sufficient numbers for individual analysis but were combined with tonsil and (base of) tongue to generate an "other HPV-Associated" subset (Fig. 3a).

Regarding survival driven by oropharyngeal tumors, "other HPV-associated" and non-HPV-associated stratification reveals three distinct outcomes in non-Appalachia (Fig. 3h): "Other HPV-associated" $(n=18,627 / 33,347 ; \mathrm{CRS}=59.7 \%)$ has the best outcome, followed by oropharynx $(n=1,833 / 33,347 ; C R S=37.5 \%)$, then non-HPV-associated ( $n=7,856 / 33,347 ; \mathrm{CRS}=30.9 \%)$. However, in Appalachia, only two distinct outcomes are revealed: "Other HPV-associated" ( $n=3,129 / 33,347 ; \mathrm{CRS}=56.7 \%)$ has the best outcome, whereas oropharynx $(n=325 / 33,347 ; \quad C R S=24.4 \%)$, has a statistically similar outcome to non-HPV-associated $(n=1,577 / 33,347 ; C R S=29.5 \%)$ (Fig. 3h). These results suggest that the observed Appalachian survival disparity is largely driven by lower survival in the oropharynx subregion of HPV-associated stage IV male oropharyngeal patients.

\section{High Appalachian Tobacco Use Correlates with Stage IV White Appalachian Male OC/P outcomes}


The lower survival of stage IV white Appalachian male HPV-associated cancer compared to confirmed HPVpositive national cases implies that risk factors other than HPV are driving poorer survival in the Appalachian cohort (Fig. 3b). Since smoking and smokeless tobacco use are highest in Appalachia[19], and current smoker prevalence rates have remained higher in West Virginia (a state with all counties within Appalachia) than the nation for several decades[36] (Fig. 4a), we evaluated tobacco use prevalence rates in Appalachian states from state BRFSS registries stratified by Appalachian residency and sex using data from 2016 as a representative year.

In males, smoking prevalence rates were significantly higher in Appalachian counties compared to nonAppalachian counties, $\mathrm{P}=4.441 \mathrm{e}-16$ and non-Appalachian states, $\mathrm{P}<2.2 \mathrm{e}-16$. Specifically, male smoking prevalence rates were significantly higher in Appalachian counties compared to non-Appalachian counties in Kentucky $(P=2.80 e-04)$, New York $(P=2.55 e-04)$, Ohio $(P=0.02965)$, and Virginia $(P=0.008904)$ (Fig. 4b, top). Male smokeless tobacco prevalence rates were significantly higher in Appalachian counties compared to nonAppalachian counties, $\mathrm{P}<2.2 \mathrm{e}-16$ and non-Appalachian states, $\mathrm{P}<2.2 \mathrm{e}-16$. Specifically, male smokeless tobacco prevalence rates were significantly higher in Appalachian counties compared to non-Appalachian counties in Kentucky $(P<2.2 e-16)$, New York $(P=9.03 e-7)$, North Carolina $(P=5.44 e-6)$, Ohio $(P=1.87 e-12)$, South Carolina $(P=0.00419)$, and Virginia $(P=6.63 e-9)$. Significant differences were also observed in Maryland and Pennsylvania, but these regions had fewer than 50 respondents.

West Virginia contains no non-Appalachian counties for comparison, but the smoking tobacco rate of $25.8 \%$ and the smokeless tobacco rate of $15.9 \%$ are similar to that of other states with significant differences in the region (Fig. 4b, top). Interestingly, except for Alabama, states with significantly higher male smokeless tobacco use include every state with a significant difference in CRS for stage IV white Appalachian male OC/P and HPV-associated cancers (Fig. 4d).

In females, average smoking prevalence rates were higher in Appalachian counties compared to nonAppalachian counties, $\mathrm{P}<2.2 \mathrm{e}-16$, and non-Appalachian states, $\mathrm{P}<2.2 \mathrm{e}-16$ (Fig. 4b, bottom) and were statistically different than that of Appalachian males (20.7\% males vs. $19.2 \%$ females) (Fig. 4c). Smoking prevalence rates were significantly higher in Appalachian counties compared to non-Appalachian counties in Alabama $(P=1.5 e-4)$, Kentucky $(P=1.33 e-9)$, Maryland $(P=0.02284)$, New York $(P=3.4 e-9)$, Ohio $(P=9.38 e-5)$, 
Pennsylvania $(P=1.44 \mathrm{e}-4)$, and Virginia $(P=0.0362)$. Female smokeless tobacco prevalence rates were significantly higher in Appalachian counties compared to non-Appalachian counties, $\mathrm{P}=8.76 \mathrm{e}-4$ and nonAppalachian states, $\mathrm{P}=2.912 \mathrm{e}-05$, and were significantly different than that of Appalachian males (10.9\% males vs. $1.2 \%$ females), $\mathrm{P}<2.2 \mathrm{e}-16$ (Fig. 4c). Significant differences were observed in Kentucky and Maryland, although these regions had fewer than 50 respondents. These data indicate that significantly higher smoking and smokeless tobacco rates within Appalachia correlate with disparate survival of stage IV white Appalachian male OC/P patients in most Appalachian disparity states. 


\section{Discussion}

With a majority of population and geographical coverage, our direct, non-exploratory analysis of HNSCC survival from available states representing all ARC-defined Appalachian subregions[16] identifies stage IV white Appalachian males with OC/P cancer as having lower cancer-related survival compared to non-Appalachian males. Based on available data, this stage- and sex-specific disparity is manifest within the Appalachian areas of four states. The disparity is restricted to the ICD-O-3 defined oropharynx (C10.0-10.9) within the CDC-defined HPV-associated oropharyngeal region. This disparity predominantly occurs in states with significantly higher male smoking and smokeless tobacco use, consistent with high risk-factor exposure in Appalachia known to contribute to increased HNSCC incidence[22,24,25].

Previous work evaluating Appalachian oral cancer survival using 2004 SEER data identified higher combined male and female survival in 10 states without consideration of stage or race[25]. When stratified by stage, race and sex, our multivariate analysis indicates that the only significant difference in CRS occurs in white Appalachian stage IV male OC/P patients diagnosed under AJCC-6[37]. RS values for stage IV white Appalachian and non-Appalachian male OC/P are higher than that of black males or black females, in agreement with a recognized national survival disparity for black OC/P cancers attributed to lower overall SES and cultural barriers[9,11]. In addition, female white stage IV OC/P patients have a lower CRS than male white stage IV OC/P regardless of Appalachian status, likely reflecting the lower rate of HPV-positive oropharyngeal disease in females[38,39]. Emerging national trends indicate that HPV-associated oropharyngeal cancers occur more frequently in younger, white male patients with limited tobacco use, and within rural areas[4,14,38]. These factors, coupled with blacks and white females having higher percentages of HPV-negative disease[12,13] are congruent with HPV-positive and/or HPV-associated white male oropharyngeal patients having higher survival at the national level.

OC/P patient populations consist of a mixture of HPV-negative and HPV-positive disease. White Appalachian males have a higher incidence in HPV-positive OC/P cancers, which would be expected to result in increased survival. However, stage IV-matched CRS for white Appalachian males is closer to the lower survival prevalence rates observed for white females than for non-Appalachian white males (Fig. 1c). Furthermore, male Appalachian stage IV HPV-associated oropharynx patients exhibit outcomes similar to non-HPV-associated male stage IV 
disease within and outside of Appalachia. Of the recognized cancer risk factors endemic to Appalachia, age at diagnosis, travel distance to critical care centers and disproportionate presentation of patients at stage IV have been reported to have no impact on survival in Appalachian subpopulations[40,41] (Table 1). However, increased smoking and smokeless tobacco use within Appalachia have been linked to low SES and increased HNSCC incidence[42-44], and thus are presumably factors contributing to the decreased male stage IV oropharyngeal cancer survival.

Our findings also indicate that Appalachian smoking tobacco prevalence rates, represented by West Virginia, have been higher than national averages for several decades (Fig. 4a), and while Appalachian smoking tobacco use is significantly higher in males and females, smokeless tobacco use is primarily higher in males compared to national averages (Fig. 4b). Smokeless tobacco use has been specifically linked to increased cancer risk in OC/P and oropharyngeal subsites[45-48]. Elevated smoking and smokeless tobacco use in most Appalachian states with significantly different CRS corresponds with decreased CRS in white male stage IV OC/P cancers. This has the potential to result in the male patient population garnering a higher percentage of tobacco-induced HPV-negative disease at all oral subsites, including the oropharynx. While the lack of diagnostic HPV detection by $\mathrm{p} 16$ staining as a variable in the NAACCR cohort precludes direct analysis of viral status, segregation of white male stage IV OC/P patients by non-HPV-associated OC/P and HPV-associated oropharynx indicates that HPVassociated oropharyngeal cancer is responsible for the Appalachian male stage IV disparity within ICD-O-3 oropharynx codes. The predominant subsites under this delineation are C10.0 (vallecula), C10.1 (anterior surface of epiglottis), C10.2 (lateral wall of oropharynx), C10.3 (posterior wall of pharynx) and C10.4 (branchial cleft). These oropharyngeal regions exclude palatine and lingual tonsils, as well as most other HPV-associated oropharyngeal sites containing the reticular epithelium lining the tonsillar crypts that constitute the primary sites of oropharyngeal HPV infection and neoplasia[49-51]. Higher aggregate tobacco use by Appalachian males may therefore result in more frequent carcinogen-induced malignancy at oropharynx subsites, resulting in a greater percentage of HPV-negative disease in the oropharynx and corresponding worse CRS, similar to that observed in other tobacco-heavy oropharyngeal cohorts[3,52,53].

The Appalachian-specific OC/P and oropharynx-specific disparities found in this study were diagnosed under the AJCC-6 timeframe, having clinical and pathological staging guidelines independent of HPV status. Restaging 
of HPV-positive oropharynx in 2018 under AJCC-8 due to the favorable prognosis of HPV-positive oropharyngeal cancers would shift most HPV-positive oropharynx in AJCC-6 to lower clinical stages. Such restaging would have the effect of potentially eliminating or downstaging the Appalachian male disparity described in this report. However, an additional ramification from this work is that male Appalachian or other populations with multifactorial oropharyngeal tobacco exposure may actually be under-staged using current AJCC-8 guidelines. This is an important consideration, since National Comprehensive Cancer Network (NCCN) guidelines recommend staging and treatment dependent on p16 status[52,54], where treatment de-escalation of HPV-associated oropharyngeal cancers continues to be evaluated[55-57]. Future efforts towards definitively determining the extent of HPV involvement in Appalachian OC/P and oropharynx through comprehensive p16 staining and PCR[58] will be required to better clarify the predominant factors underlying oropharynx-driven, stage-based disparities in past and future Appalachian cohorts.

\section{Limitations and Implications}

While the current study covers the majority of the Appalachian population and is the most comprehensive study of HNSCC survival of the region to date, lack of available qualified survival data from five Appalachian states prevented complete assessment of survival in the region. The association between heavy aggregate tobacco use and poor male stage IV oropharyngeal survival in states with significantly different CRS suggests that stage IV oropharyngeal patients from states with Appalachian regions containing similar tobacco use patterns may also harbor disparate outcomes. Our findings, in conjunction with the poor survival of HPV-positive oropharynx patients with high smoking histories[59,60], underscore the need for the comprehensive tobacco history of any HPV-positive oropharynx patient to be considered prior to treatment. In addition to smoking, the significantly higher smokeless tobacco use by the male Appalachian population further increases the risk of tobacco-induced cancer at HPV-associated oropharyngeal sites, leading to oropharynx tumors that are either HPV-negative, or are HPV-positive but exhibit aggressive HPV-negative tumor behavior.

\section{Conclusions}

These findings provide novel and in-depth insight into a specific demographic within a chronically underserved, rural population that is at higher clinical risk for poor OC/P outcome. Persistent high tobacco usage in Appalachia, 
in spite of increased tobacco cessation efforts, reinforces the need for continued targeted risk awareness. This includes emerging forms of supposed safer nitrosamine-containing products, such as e-cigarettes, that contribute to the cumulative patient tobacco load[61]. Inclusion of patient use of these products as standard registry variables should be considered for improved monitoring of tobacco-related disparities in future populations. The described male oropharynx disparity may also be present in male patients from other regions with heavy smoking, smokeless tobacco[62] or betel-quid[63] use, and should be monitored accordingly for similar poor survival and continued cessation intervention policies.

\section{Competing interests}

The author(s) declare no competing interests.

\section{Author Contributions}

B.W.P: Conceptualization, writing-original draft, data curation, formal analysis, and investigation. J.L.A.: Conceptualization and writing-review and editing. S.M.M.: Conceptualization, writing-review and editing. E.T.I.: Conceptualization and writing-review and editing. P.A.M.: writing-review and editing. C.J.J.: Conceptualization, writing-review and editing, supervision, methodology, software, and resources. S.A.W.: Funding acquisition, conceptualization, project administration, writing-original draft, review, editing and project supervision.

\section{Acknowledgments}

This work was supported by pilot grants from NIH U54GM104942 and P20GM103434 (B. Papenberg, J. Allen, S. Markwell, S. Weed). Mr. Johnson was supported with Federal funds from the National Cancer Institute, NIH, HHS, under Contract No. HHSN261201800006I and the Centers for Disease Control and Prevention, HHS, under Cooperative Agreement 1NU58DP006270. The content is solely the responsibility of the authors and does not necessarily represent the official views of the NIH or the Centers for Disease Control and Prevention. Additional support was provided by the West Virginia University Department of Biochemistry (S. Markwell, S. 
Weed), Department of Otolaryngology, Head and Neck Surgery (E. Interval, P. Montague) and by Transition Grant Support from the Office of Research and Graduate Education, West Virginia University Health Sciences

Center (B. Papenberg, J. Allen, S. Weed). We thank Recinda Sherman (NAACCR) and Information Management Services for assistance with IRB and database preparation, Sijin Wen (West Virginia University School of Public Health) for biostatistical validation, Pamela Moats (West Virginia University Cancer Institute Registry) and Alana Hudson (West Virginia Cancer Registry) for early conceptual help.

\section{Data Availability}

The data that support the findings of this study are available upon application and IRB approval from NAACCR, thus these data are not in the public domain. Data are however available from the authors upon reasonable request and with prior approval by NAACCR. 


\section{References}

1. Siegel, R. L., Miller, K. D. \& Jemal, A. Cancer statistics, 2020. CA. Cancer J. Clin. 70, 7-30 (2020).

2. Chow, L. Q. M. Head and neck cancer. N. Engl. J. Med. 382, 60-72 (2020).

3. Ang, K. K. et al. Human papillomavirus and survival of patients with oropharyngeal cancer. N. Engl. J. Med. 363, 24-35 (2010).

4. LeHew, C. W. et al. The health system and policy implications of changing epidemiology for oral cavity and oropharyngeal cancers in the United States from 1995 to 2016. Epidemiol. Rev. 39, 132-147 (2017).

5. Rettig, E. M. \& D'Souza, G. Epidemiology of Head and Neck Cancer. Surg. Oncol. Clin. N. Am. 24, 379396 (2015).

6. Maxwell, J. H., Grandis, J. R. \& Ferris, R. L. HPV-Associated Head and Neck Cancer: Unique Features of Epidemiology and Clinical Management. Annu. Rev. Med. 67, 91-101 (2016).

7. Marur, S. \& Forastiere, A. A. Head and Neck Squamous Cell Carcinoma: Update on Epidemiology, Diagnosis, and Treatment. Mayo Clin. Proc. 91, 386-396 (2016).

8. Spence, T., Bruce, J., Yip, K. W. \& Liu, F.-F. HPV-Associated Head and Neck Cancer. J. Natl. Cancer Inst. 107, djv344 (2015).

9. Goodwin, W. J. et al. Unequal burden of head and neck cancer in the United States. Head Neck 30, 358-371 (2008).

10. Morse, D. E. \& Kerr, A. R. Disparities in oral and pharyngeal cancer incidence, mortality and survival among black and white Americans. J. Am. Dent. Assoc. 137, 203-212 (2006).

11. Daraei, P. \& Moore, C. E. Racial Disparity Among the Head and Neck Cancer Population. J. Cancer Educ. 30, 546-551 (2015).

12. Settle, K. et al. Racial survival disparity in head and neck cancer results from low prevalence of human 
papillomavirus infection in black oropharyngeal cancer patients. Cancer Prev. Res. 2, 776-781 (2009).

13. Saba, N. F. et al. Gender and ethnic disparities in incidence and survival of squamous cell carcinoma of the oral tongue, base of tongue, and tonsils: A surveillance, epidemiology and end results programbased analysis. Oncology 81, 12-20 (2011).

14. Siegel, R. L., Miller, K. D. \& Jemal, A. Cancer statistics, 2019. CA. Cancer J. Clin. 69, 7-34 (2019).

15. Zahnd, W. E. et al. Rural-Urban differences in cancer incidence and trends in the United States. Cancer Epidemiol. Biomarkers Prev. 27, 1265-1274 (2018).

16. The Appalachian Region - Appalachian Regional Commission.

https://www.arc.gov/appalachian_region/TheAppalachianRegion.asp.

17. Pollard, K. \& Jacobsen, L. A. The Appalachian Region: A Data Overview from the 2013-2017 American Community Survey - Appalachian Regional Commission.

https://www.arc.gov/research/researchreportdetails.asp?REPORT_ID=159 (2019).

18. Viens, L. J. et al. Human Papillomavirus-Associated Cancers - United States, 2008-2012. MMWR. Morb. Mortal. Wkly. Rep. 65, 661-666 (2016).

19. Behavioral Risk Factor Data: Tobacco Use (2011 to present) | Chronic Disease and Health Promotion Data \&amp; Indicators. https://chronicdata.cdc.gov/Survey-Data/Behavioral-Risk-Factor-Data-TobaccoUse-2011-to-pr/wsas-xwh5.

20. Knox, S., Basu, S. \& Remick, S. A Systems Approach to Cancer Health Disparities in Appalachia. Austin J Public Heal. Epidemiol 1, 1004 (2014).

21. Paskett, E. D. et al. Disparities in underserved white populations: the case of cancer-related disparities in Appalachia. Oncologist 16, 1072-81 (2011).

22. Lengerich, E. J. et al. Cancer incidence in Kentucky, Pennsylvania, and West Virginia: Disparities in Appalachia. J. Rural Heal. 21, 39-47 (2004). 
23. Reiter, P. L. et al. Assessing the burden of HPV-related cancers in Appalachia. Hum. Vaccines Immunother. 9, 90-96 (2013).

24. Wilson, R. J., Ryerson, A. B., Singh, S. D. \& King, J. B. Cancer Incidence in Appalachia, 2004-2011. Cancer Epidemiol. Biomarkers Prev. 25, 2004-2011 (2016).

25. Bruce C. Casto et al. Oral Cancer in Appalachia. J. Health Care Poor Underserved 20, 274-285 (2009).

26. County Economic Status in Appalachia, FY 2017 - Appalachian Regional Commission. https://www.arc.gov/research/MapsofAppalachia.asp?MAP_ID=116.

27. Cancer in North America: 2009-2013, Volume Four: Cancer Survival in the United States and Canada 2006-2012. (2013).

28. Measures of Cancer Survival. https://surveillance.cancer.gov/survival/measures.html.

29. Cartographic Boundary Files - Shapefile / US Census Bureau.

https://www.census.gov/geographies/mapping-files/time-series/geo/carto-boundary-file.2014.html.

30. QGIS Development Team (2019). QGIS Geographic Information System. Open Source Geospatial Foundation Project. http://qgis.osgeo.org.

31. Cerami, E. et al. The cBio Cancer Genomics Portal: An open platform for exploring multidimensional cancer genomics data. Cancer Discov. 2, 401-404 (2012).

32. Gao, J. et al. Integrative analysis of complex cancer genomics and clinical profiles using the cBioPortal. Sci. Signal. 6, 1-34 (2013).

33. Van Dyne, E. A. et al. Trends in Human Papillomavirus-Associated Cancers - United States, 19992015. MMWR. Morb. Mortal. Wkly. Rep. 67, 918-924 (2018).

34. Centers for Disease Control and Prevention (CDC). Methodologic changes in the Behavioral Risk Factor Surveillance System in 2011 and potential effects on prevalence estimates. MMWR. Morb. Mortal. Wkly. Rep. 61, 410-3 (2012). 
35. Annual Estimates of the Resident Population: April 1, 2010 to July 1, 2016 | 2015 Population Estimates | US Census Bureau.

https://factfinder.census.gov/faces/tableservices/jsf/pages/productview.xhtml?src=bkmk.

36. West Virginia Behavioral Risk Factor Surveillance System.

http://www.wvdhhr.org/bph/hsc/pubs/Topics/default.shtml.

37. Lydiatt, W. M. et al. Head and Neck cancers-major changes in the American Joint Committee on cancer eighth edition cancer staging manual. CA. Cancer J. Clin. 67, 122-137 (2017).

38. Mahal, B. A. et al. Incidence and Demographic Burden of HPV-associated Oropharyngeal Head and Neck Cancers in the United States. Cancer Epidemiol. Biomarkers Prev. cebp.0038.2019 (2019) doi:10.1158/1055-9965.epi-19-0038.

39. Lawrence, M. S. et al. Comprehensive genomic characterization of head and neck squamous cell carcinomas. Nature 517, 576-582 (2015).

40. Ringstrom, M. J. et al. Travel distance: Impact on stage of presentation and treatment choices in head and neck cancer. Am. J. Otolaryngol. - Head Neck Med. Surg. 39, 575-581 (2018).

41. Gleich, L. L. et al. Therapeutic Decision Making in Stages III and IV Head and Neck Squamous Cell Carcinoma. Arch. Otolaryngol. Neck Surg. 129, 26 (2003).

42. Stanford-Moore, G. et al. Interaction between known risk factors for head and neck cancer and socioeconomic status: the Carolina Head and Neck Cancer Study. Cancer Causes Control 29, 863-873 (2018).

43. Welte, J. W., Barnes, G. M., Tidwell, M. C. O. \& Hoffman, J. H. Tobacco use, heavy use, and dependence among adolescents and young adults in the United States. Subst. Use Misuse 46, 10901098 (2011).

44. Nemeth, J. M. et al. Factors influencing smokeless tobacco use in rural Ohio Appalachia. J. Community Health 37, 1208-1217 (2012). 
45. Lee, P. N. \& Hamling, J. Systematic review of the relation between smokeless tobacco and cancer in Europe and North America. BMC Med. 7, 36 (2009).

46. Zhou, J. et al. Smokeless tobacco and risk of head and neck cancer: Evidence from a case-control study in New England. Int. J. Cancer 132, 1911-1917 (2013).

47. Wyss, A. B. et al. Smokeless tobacco use and the risk of head and neck cancer: Pooled analysis of US studies in the inhance consortium. Am. J. Epidemiol. 184, 703-716 (2016).

48. Mello, F. W. et al. The synergistic effect of tobacco and alcohol consumption on oral squamous cell carcinoma: a systematic review and meta-analysis. Clin. Oral Investig. 23, 2849-2859 (2019).

49. Gillison, M. L. Evidence for a Causal Association Between Human Papillomavirus and a Subset of Head and Neck Cancers. J. Natl. Cancer Inst. 92, 709-720 (2000).

50. Kim, S. H. et al. HPV integration begins in the tonsillar crypt and leads to the alteration of p16, EGFR and c-myc during tumor formation. Int. J. Cancer 120, 1418-1425 (2007).

51. Thavaraj, S. et al. Patients with HPV-related tonsil squamous cell carcinoma rarely harbour oncogenic HPV infection at other pharyngeal sites. Oral Oncol. 50, 241-246 (2014).

52. Kompelli, A. R. et al. Prognostic Impact of High-Risk Pathologic Features in HPV-Related Oropharyngeal Squamous Cell Carcinoma and Tobacco Use. Otolaryngol. - Head Neck Surg. (United States) 160, 855-861 (2019).

53. Grønhøj, C. et al. Impact on survival of tobacco smoking for cases with oropharyngeal squamous cell carcinoma and known human papillomavirus and p16-status: a multicenter retrospective study. Oncotarget 10, 4655-4663 (2019).

54. National Comprehensive Cancer Network. Head and Neck Cancers (Version 3.2019). https://www.nccn.org/professionals/physician_gls/pdf/head-and-neck.pdf.

55. Oosthuizen, J. C. \& Kinsella, J. B. Is treatment de-escalation a reality in HPV related oropharyngeal cancer? Surgeon 14, 180-183 (2016). 
56. White, R. et al. Practice patterns and outcomes following radiation dose de-escalation for oropharyngeal cancer. Laryngoscope 1-6 (2019) doi:10.1002/lary.28083.

57. Oosthuizen, J. C. \& Doody, J. De-intensified treatment in human papillomavirus-positive oropharyngeal cancer. Lancet 393, 5-7 (2019).

58. Rietbergen, M. M. et al. Molecular characterization of p16-immunopositive but HPV DNA-negative oropharyngeal carcinomas. Int. J. Cancer 134, 2366-2372 (2014).

59. Haigentz, M. et al. Understanding Interactions of Smoking on Prognosis of HPV-Associated Oropharyngeal Cancers. Adv. Ther. 35, 255-260 (2018).

60. Liskamp, C. P. et al. Adverse effect of smoking on prognosis in human papillomavirus-associated oropharyngeal carcinoma. Head Neck 38, 1780-1787 (2016).

61. Cummings, K. M. et al. E-cigarettes and cancer patients. J. Thorac. Oncol. 9, 438-441 (2014).

62. Critchley, J. A. \& Unal, B. Health effects associated with smokeless tobacco: A systematic review. Thorax 58, 435-443 (2003).

63. Tangjaturonrasme, N., Vatanasapt, P. \& Bychkov, A. Epidemiology of head and neck cancer in Thailand. Asia. Pac. J. Clin. Oncol. 14, 16-22 (2018). 
a

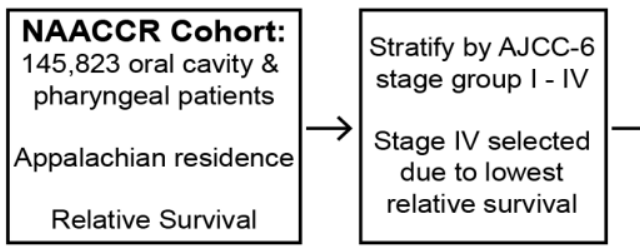

b

All Sexes, All Races, All Stages

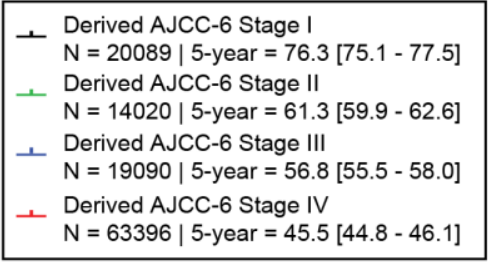

At 60 Months:

- $p<0.00001=p<0.00001$

$=0.00002$

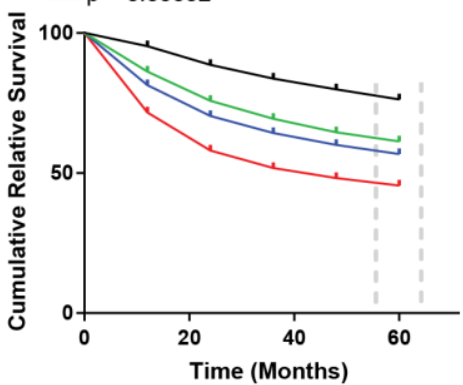

C

\begin{tabular}{|c|c|}
\hline+ & $\begin{array}{l}\text { Non-Appalachian White Males } \\
\mathrm{N}=37843 \mid 5 \text {-year }=49.2[48.2-50.2] \\
\text { Appalachian White Males } \\
\mathrm{N}=5014 \mid 5 \text {-year }=44.9[42.0-47.8]\end{array}$ \\
\hline - & $\begin{array}{l}\text { Non-Appalachian White Females } \\
\mathrm{N}=9458 \mid 5 \text {-year }=43.7[42.3-45.2] \\
\text { Appalachian White Females } \\
\mathrm{N}=1381 \mid 5 \text {-year }=43.1[39.3-46.8]\end{array}$ \\
\hline
\end{tabular}

$$
\text { At } 60 \text { Months: }
$$$$
=\mathrm{p}=0.00001=\text { n.s. }
$$

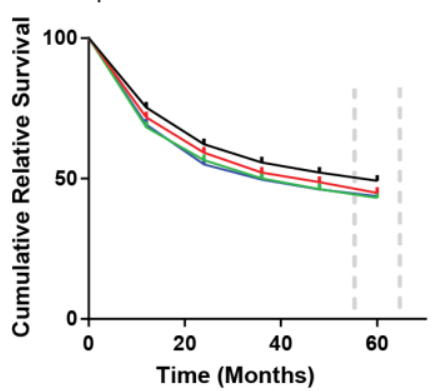

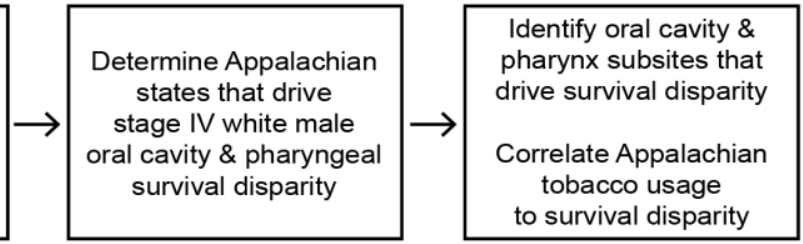

d

Stage IV Black Patients

\begin{tabular}{|c|c|}
\hline+ & $\begin{array}{l}\text { Non-Appalachian Black Males } \\
\mathrm{N}=5297 \mid \text { 3-year }=34.4[32.4-36.5] \\
\text { Appalachian Black Males } \\
\mathrm{N}=387 \mid \text { 3-year }=35.8[28.6-43.1]\end{array}$ \\
\hline+ & $\begin{array}{l}\text { Non-Appalachian Black Females } \\
\mathrm{N}=1540 \mid \text { 3-year }=34.8[31.4-38.2] \\
\text { Appalachian Black Females } \\
\mathrm{N}=116 \mid \text { 3-year }=46.1[33.7-57.6]\end{array}$ \\
\hline
\end{tabular}

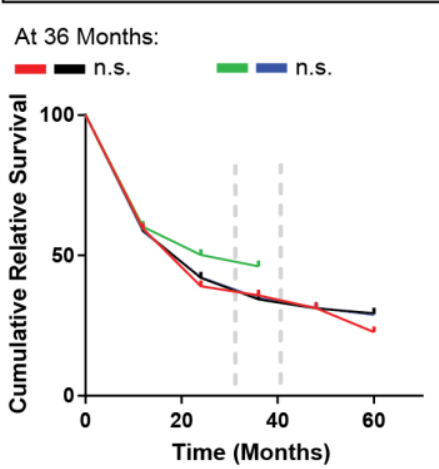

Figure 1. Identification of a Survival Disparity in White Appalachian Male Stage IV OC/P cancer. A. Flow diagram of procedures used to evaluate Appalachian-specific survival data. Boxes contain rationale and steps involved in the sequential stratification and cohort analysis for OC/P cancer. B. Survival analysis of Appalachian OC/P cancer stratified by clinical stage. CRS values are plotted for each year after diagnosis, with five-year (60 month) ratios evaluated across all AJCC-6 stages. C and D. Survival analysis of white (C) and black (D) stage IV OC/P cancer stratified by Appalachian residency and sex. CRS values are plotted as in (B). Patient N, fiveyear CRS with 95\% Cl and P-values between significant groups are shown at the top of each graph; n.s., not significant. Black patients were evaluated for significance at 36 months due to lack of complete female survival by 48 months and beyond. 
a

\begin{tabular}{|l|} 
Stage IV White Males \\
\hline$+\quad$ Non-Appalachian States \\
$\mathrm{N}=28143 \mid$ 5-year $=49.6[48.5-50.7]$ \\
+ Appalachian States Non-Appalachian Counties \\
$\mathrm{N}=9700 \mid 5-y e a r=47.9[45.9-49.9]$ \\
$+\quad$ Appalachian States Appalachian Counties \\
$\mathrm{N}=5014 \mid$ 5-year $=44.9[42.0-47.8]$
\end{tabular}

At 60 Months:

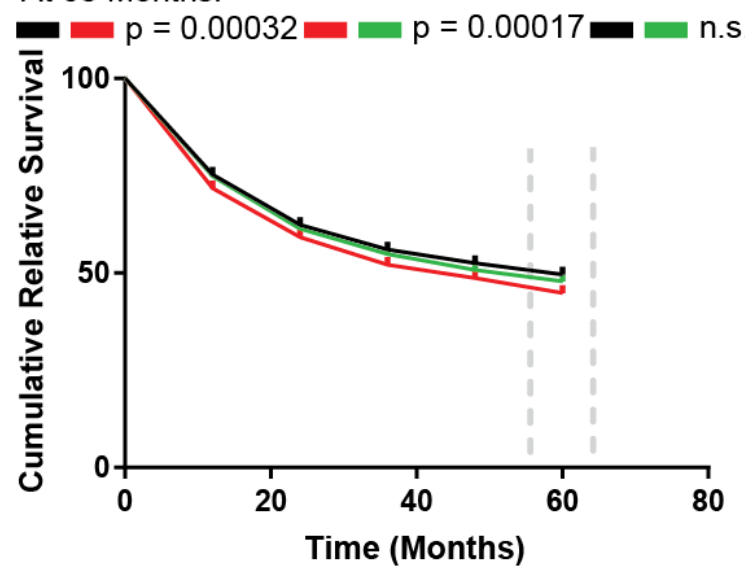

C

\begin{tabular}{|l|}
\multicolumn{1}{|c|}{ Stage IV White Males } \\
\hline$+\quad$ Non-Appalachian States \\
$\mathrm{N}=28143$ | 5-year = 49.6 [48.5 - 50.7] \\
$+\quad$ Alabama Appalachian Counties \\
$\mathrm{N}=626$ | 5-year = 39.7 [ 33.5 - 45.7] \\
Kentucky Appalachian Counties \\
$\mathrm{N}=372$ | 5-year = 39.6 [29.1 - 50.0] \\
+ Pennsylvania Appalachian Counties \\
$\mathrm{N}=1695$ | 5-year = 45.3 [40.6 - 50.0] \\
West Virginia Appalachian Counties \\
$\mathrm{N}=587$ | 5-year = 35.3 [27.0 - 43.6]
\end{tabular}

At 60 Months:

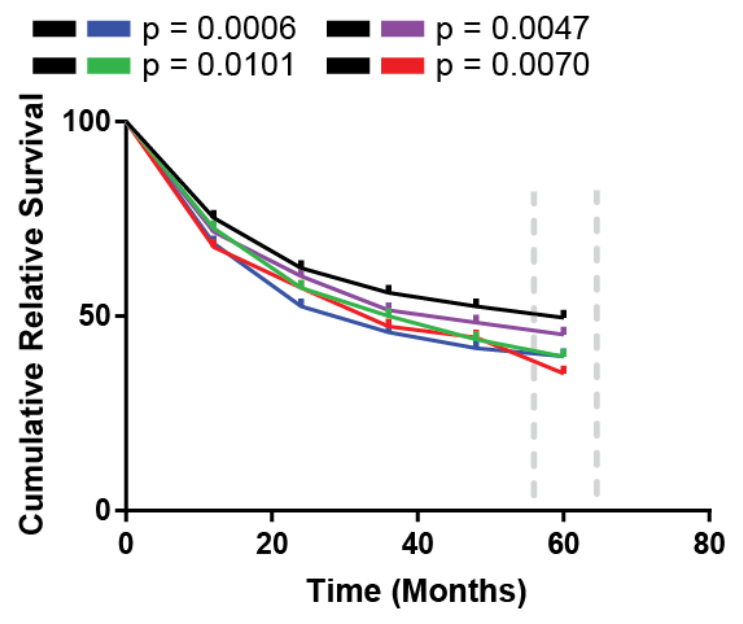

b

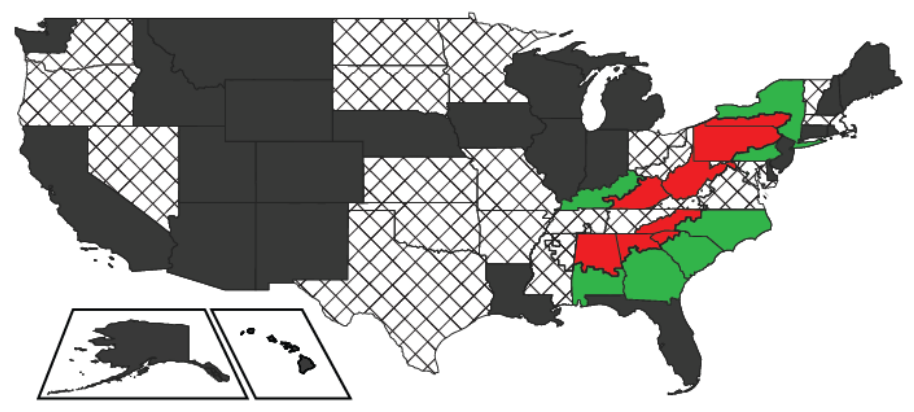

\begin{tabular}{|c|}
\hline Appalachian States - Appalachian Counties \\
\hline Appalachian States - Non-Appalachian Counties \\
\hline Non-Appalachian States \\
\hline Data Not Available \\
\hline
\end{tabular}

d

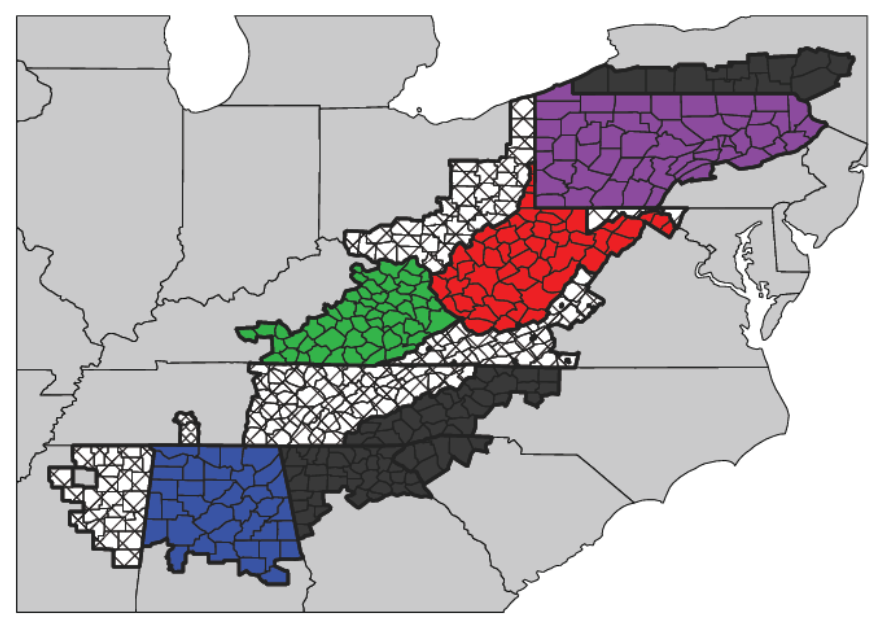

Significantly Different Appalachian States

Non-Significantly Different Appalachian States

Data Not Available

Figure 2. State-level analysis of Appalachian OC/P survival. A. Disparate stage IV white Appalachian male OC/P survival is restricted to Appalachian counties. Plotted CRS values over time are shown for nonAppalachian states (black), non-Appalachian counties within Appalachian states (green) and Appalachian 
counties within Appalachian states (red). Patient N, five-year CRS with $95 \% \mathrm{Cl}$ and P-values at 60 months are shown at the top of the graph; n.s., not significant. B. Mapping of the denoted geographic regions evaluated in (A). C. Select Appalachian states with significantly different CRS values contain a survival disparity in OC/P cancer. Appalachian states with significant CRS values are shown plotted over time. Patient N, five-year CRS with $95 \% \mathrm{Cl}$ and P-values at 60 months for states with significant survival differences between Appalachian and non-Appalachian counties are shown at the top of the graph. D. Map of Appalachian region displaying states with significantly different stage IV white Appalachian male OC/P CRS. States with significant survival differences between Appalachian and non-Appalachian counties are in black, states with no available data are marked with a cross-hatched pattern. 


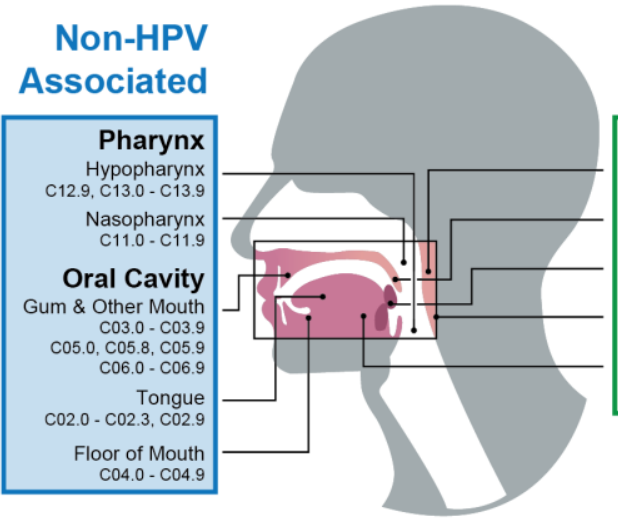

HPV

Associated

\begin{tabular}{|l|}
\hline Oropharynx \\
Oropharynx \\
C10.0 - C10.9 \\
\hline Gum \& Other Mouth \\
Co5.1, Co5.2 \\
Tonsil \\
C09.0 - c09.9 \\
Other Oral Cavity \& Pharynx \\
C14.0 - C14.8 \\
Tongue \\
c01.9, C02.4, c02.8 \\
\hline
\end{tabular}

Other HPV

Associated

C

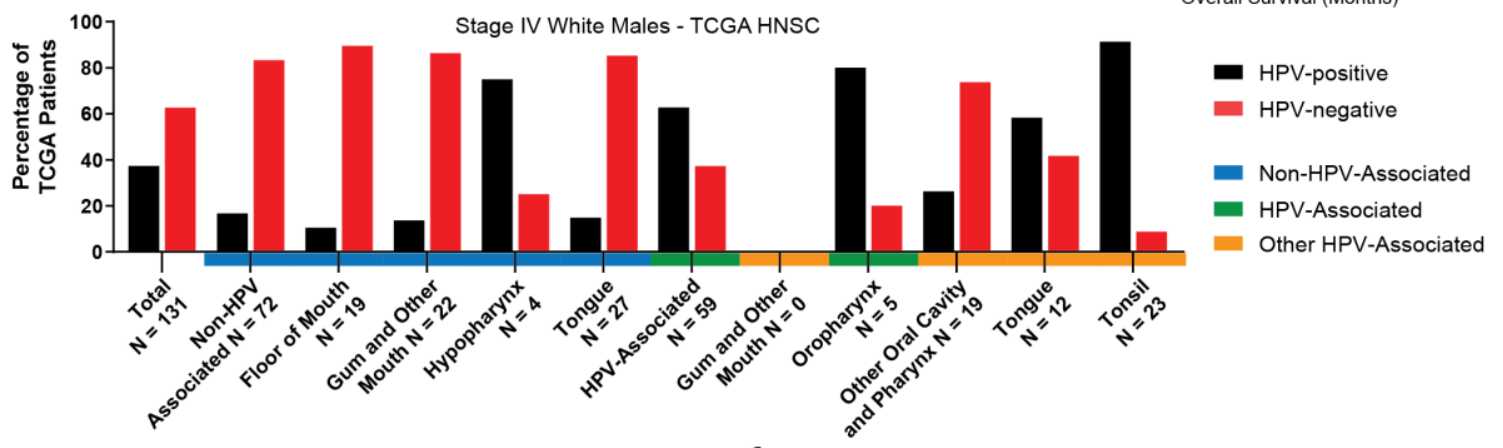

d Stage IV White Males

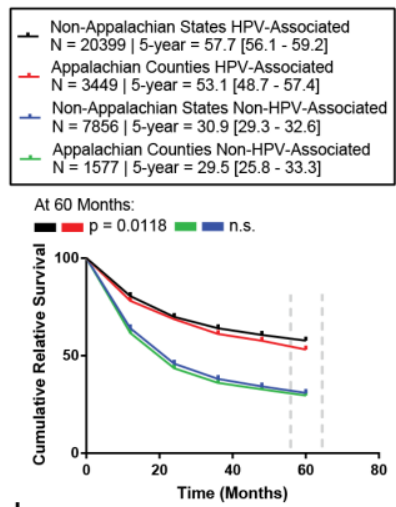

$\mathrm{h}$

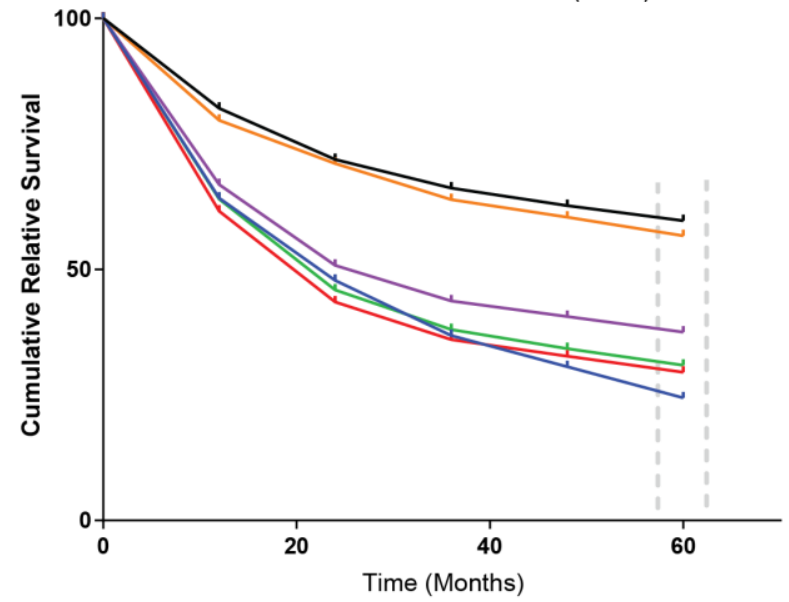

e $\begin{gathered}\text { Stage IV White Males } \\ \text { Oropharynx (C10.0-C10.9) }\end{gathered}$

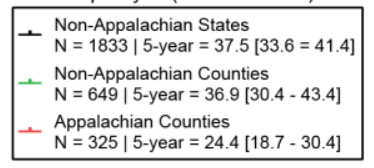

At 60 Months:

- $\mathrm{p}=0.0394=\mathrm{p}=0.0399$

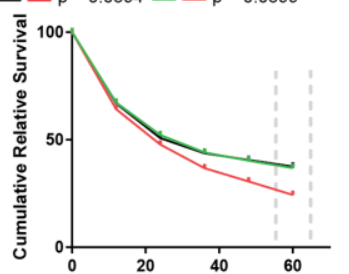

20
Time (Months) f Stage IV White Males Tonsil (C09.0 - C09.9)

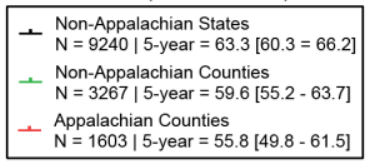
At 60 Months:

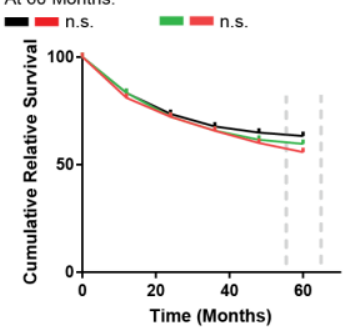

g Stage IV White Males

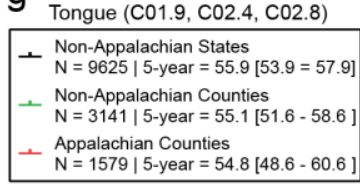
At 60 Months:

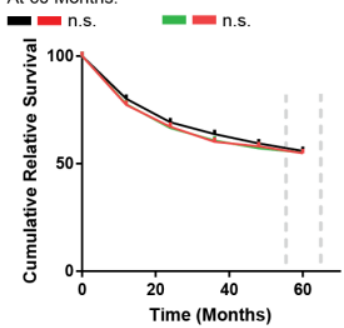

+ Non-Appalachian States Oropharynx $\mathrm{N}=1833$ | 5-year $=37.5[33.6-41.4]$

+ Non-Appalachian States Other HPV-Associated $\mathrm{N}=18627$ | 5-year $=59.7[58.0-61.4]$

_ Non-Appalachian States Non-HPV-Associated $\mathrm{N}=7856$ | 5-year $=30.9[29.3-32.6]$

_. Appalachian Counties Oropharynx $\mathrm{N}=325$ | 5-year $=24.4[18.7-30.4]$

Appalachian Counties Other HPV-Associated $\mathrm{N}=3129 \mid 5$-year $=56.7[51.9-61.3]$

Appalachian Counties Non-HPV-Associated $\mathrm{N}=1577 \mid 5$-year $=29.5[25.8-33.3]$

At 60 Months:

$$
\begin{aligned}
& -p<0.00001 \\
& -p . s . \\
& =p=0.0394 \quad-p<0.00001 \\
& =\text { n.s. }
\end{aligned}
$$

Figure 3. The Stage IV White Appalachian Male OC/P survival disparity is predominant in HPV-associated

oropharyngeal cancers. A. Schematic of the head and neck showing location of non-HPV-associated pharynx 
and oral cavity (blue), and CDC-defined HPV-associated oropharynx (green) cancers. A subset of the CDCdefined HPV-associated oropharynx is denoted in orange. ICD-O-3 site groups and corresponding site codes for each cancer type are indicated (see Materials \& Methods for detailed description of each site group). B. Overall survival between confirmed and HPV-associated OC/P subtypes. Kaplan-Meier analysis of overall survival of stage IV white males from the TCGA HNSC cohort stratified by HPV status (HPV-positive or HPV-negative) or by ICD-O-3-coded HPV association (HPV-associated and non-HPV-associated). P-values calculated using Mantel-Cox log-rank test, patient N, median and five-year overall survival for each group are noted. C. HPV status of OC/P subsites in stage IV white male TCGA patients. Percent of patients with confirmed HPV-negative (red) or HPV-positive (black) disease for each indicated subsite is shown. Denoted subsite groupings are indicated at the bottom as non-HPV-associated (blue), oropharynx (green), or other HPV-associated (orange). D. The stage IV white Appalachian male OC/P survival disparity is present in HPV-associated oropharyngeal cancer. CRS values are plotted over time for stage IV white Appalachian male OC/P patients stratified by Appalachian and non-Appalachian counties and HPV- or non-HPV-associated status. E-G. Stage IV white males stratified by HPV-associated oropharynx subsites. Oropharynx (E), tonsil (F), and tongue (G). CRS values are plotted over time with patients stratified by Appalachian counties (red), non-Appalachian counties (green) and non-Appalachian states (black). H. Stage IV white males stratified by non-HPV-associated, oropharynx, other HPV-associated subsites and Appalachian residency. For D-H, RS values are plotted over time with patients stratified by Patient N, five-year CRS with $95 \% \mathrm{Cl}$ and $\mathrm{P}$-values at 60 months for each group are shown at the top of each respective graph; n.s., not significant. 


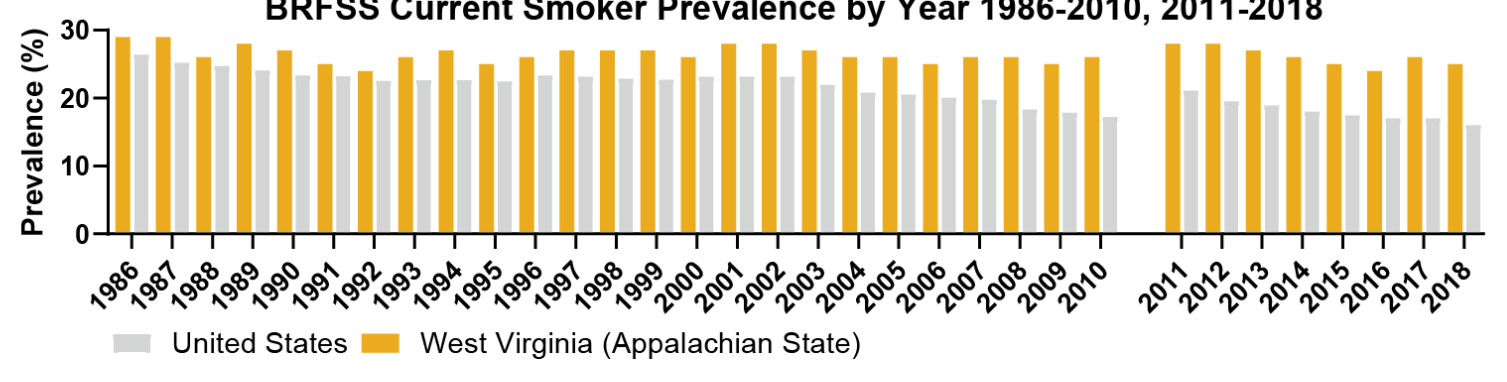

b BRFSS Current Smoker and Smokeless Tobacco Use Prevalence by Region and Sex, 2016 \begin{tabular}{|l} 
Appalachian States, Appalachian Counties Smoking \\
$\begin{array}{l}\text { Appalachian States, Non-Appalachian Counties Smoking } \\
\text { Non-Appalachian States Smoking }\end{array}$ \\
$\begin{array}{l}\text { Nalachian States, Non-Appalachian Counties Smokeless } \\
\text { Non-Appalachian States Smokeless }\end{array}$
\end{tabular}
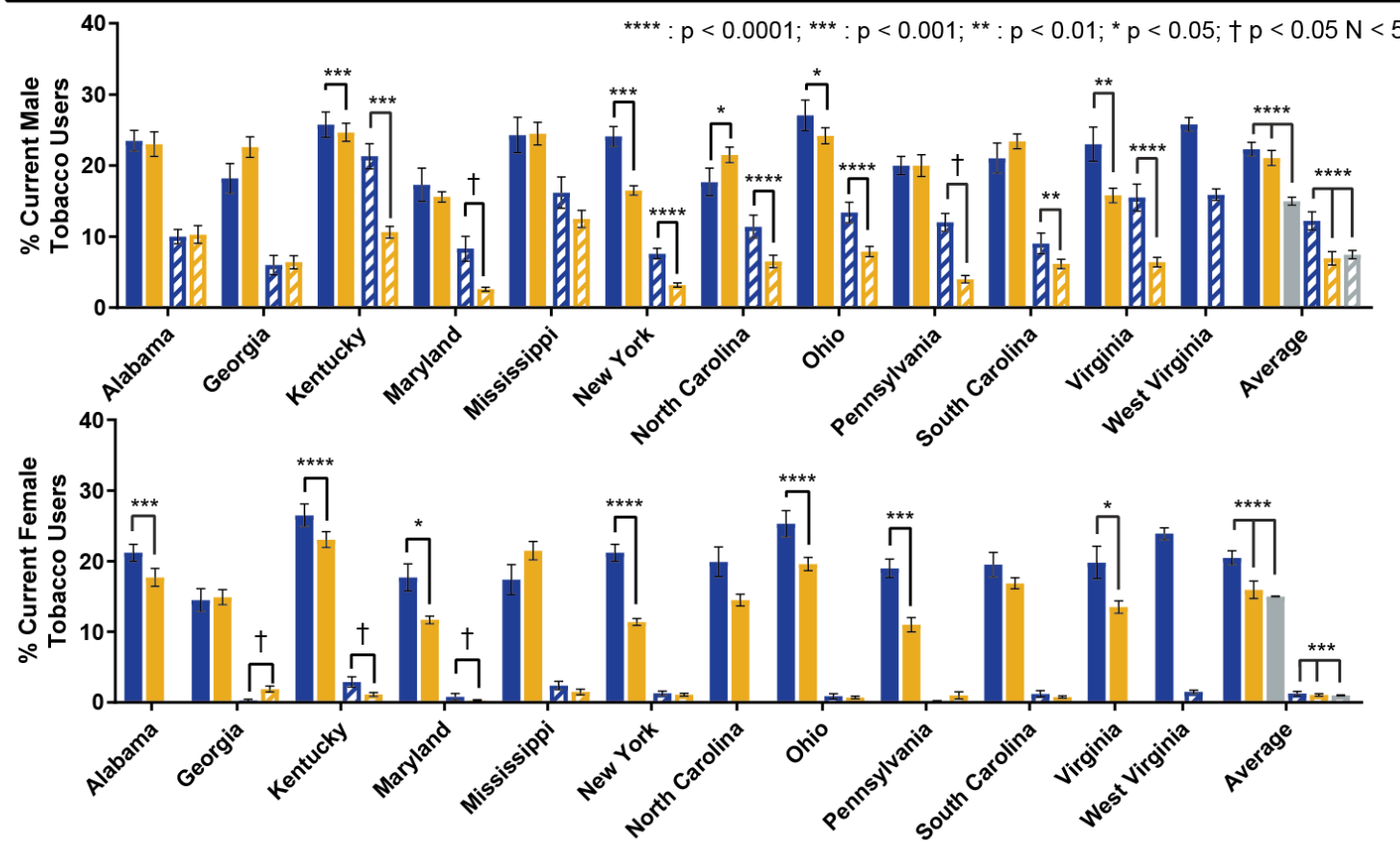

\section{BRFSS Current Smoker and Smokeless Tobacco Use Prevalence by Region and Sex, 2016}
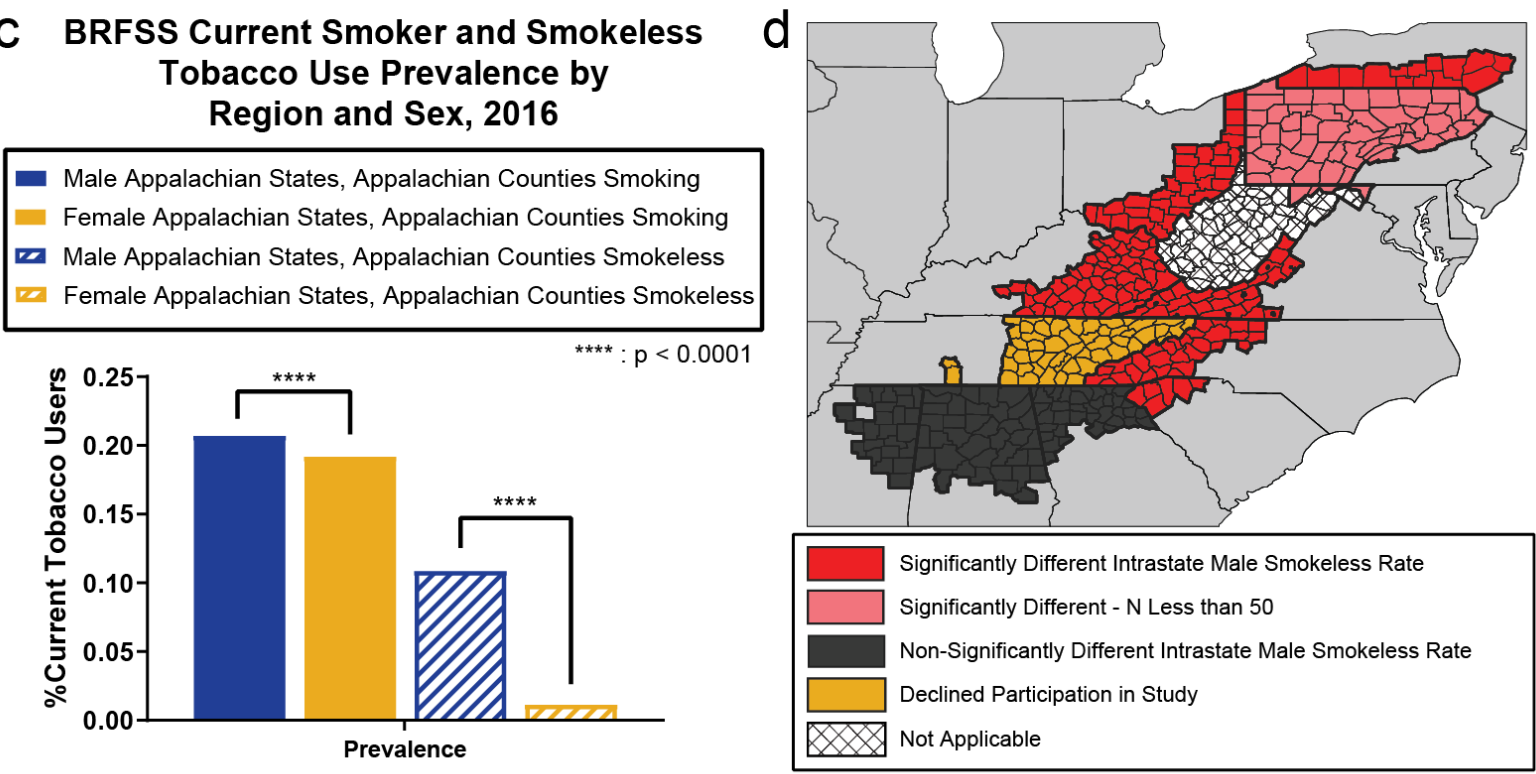
Figure 4. High combined tobacco use in Stage IV white Appalachian male OC/P disparity states. A. Overall smoking prevalence rates in West Virginia (as a representation of Appalachia, gold) compared to the United States (grey). Years 2011-2018 cannot be directly compared to earlier years due to differences in weighting methodology across time. B. Elevated tobacco use by Appalachian males (top) and females (bottom). Data for 2016 smoking weighted prevalence estimates for males in Appalachian states stratified by county status (blue, Appalachian counties; gold, non-Appalachian counties; grey, non-Appalachian states), smoking (solid) or smokeless (cross-hatched) tobacco use with P-values calculated using G-test for independence without Yates' correction are shown at top. Error bars represent $95 \%$ Cl. C. Combined Appalachia county smoking (solid) and smokeless (cross-hatched) weighted prevalence estimates with P-value calculated using G-test for independence without Yates' correction are shown above significant results. D. Mapping of Appalachian counties smokeless tobacco use. Appalachian counties with significantly higher (red) and non-significantly different (black) smokeless tobacco use are shown compared to non-Appalachian counties within each respective Appalachian state. West Virginia (hatched; non-applicable) lacks non-Appalachian counties for comparison. 


\begin{tabular}{|c|c|c|c|c|c|c|c|}
\hline $\begin{array}{l}2007 \text { - } 2013 \text { NAACCR } \\
\text { Cohort Characteristicst }\end{array}$ & $\begin{array}{l}\text { Total OC/P and } \\
\text { Laryngeal } \\
\mathrm{n}=\mathbf{2 1 4 8 2 1}(\mathbf{1 0 0 \% )}\end{array}$ & $\begin{array}{l}\text { Total OC/P } \\
n=154525(100 \%)\end{array}$ & $\begin{array}{l}\text { Appalachian OC/P } \\
n=16366(10.59 \%)\end{array}$ & $\begin{array}{l}\text { Non-Appalachian OC/P } \\
n=138159(89.41 \%)\end{array}$ & $\begin{array}{l}\text { Total Laryngeal } \\
\mathrm{n}=60296(100 \%)\end{array}$ & $\begin{array}{l}\text { Appalachian } \\
\text { Laryngeal } \\
\mathrm{n}=7614(12.63 \%) \\
\end{array}$ & $\begin{array}{l}\text { Non-Appalachian } \\
\text { Laryngeal } \\
\mathbf{n}=52682(87.37 \%)\end{array}$ \\
\hline & Mean \pm SD & Mean \pm SD & Mean \pm SD & Mean \pm SD & Mean \pm SD & Mean \pm SD & Mean \pm SD \\
\hline \multirow[t]{2}{*}{ Age (years) } & $63.05 \pm 12.01$ & $62.35 \pm 12.22$ & $61.99 \pm 12.05$ & $62.39 \pm 12.24$ & $64.83 \pm 11.26$ & $63.73 \pm 11.10$ & $64.99 \pm 11.28$ \\
\hline & CRS \pm SEM & CRS \pm SEM & CRS \pm SEM & CRS \pm SEM & CRS \pm SEM & CRS \pm SEM & CRS \pm SEM \\
\hline \multirow[t]{2}{*}{ Relative Survival (\%) } & $56.9 \pm 0.2$ & $55.0 \pm 0.2$ & $52.7 \pm 0.7$ & $55.3 \pm 0.2$ & $60.1 \pm 0.4$ & $58.1 \pm 1.1$ & $60.4 \pm 0.4$ \\
\hline & n (\%) & n (\%) & n (\%) & $\mathrm{n}(\%)$ & $\mathrm{n}(\%)$ & n (\%) & n (\%) \\
\hline \multicolumn{8}{|l|}{ Sex } \\
\hline Female & $54,713(25.5)$ & $42,625(27.6)$ & $4,587(28)$ & $38,038(27.5)$ & $12,088(20)$ & $1,593(20.9)$ & $10,495(19.9)$ \\
\hline \multicolumn{8}{|l|}{ Race } \\
\hline White & $183,029(85.2)$ & $132,955(86)$ & $15,049(92)$ & $117,906(85.3)$ & $50,074(83)$ & $6,892(90.5)$ & $43,182(82)$ \\
\hline Black & $23,385(10.9)$ & $14,769(9.6)$ & $1,123(6.9)$ & $13,646(9.9)$ & $8,616(14.3)$ & $661(8.7)$ & $7,955(15.1)$ \\
\hline Other & $8,407(3.9)$ & $6,801(4.4)$ & $194(1.2)$ & $6,607(4.8)$ & $1,606(2.7)$ & $61(0.8)$ & $1,545(2.9)$ \\
\hline \multicolumn{8}{|l|}{ AJCC-6 Stage } \\
\hline I & $40,356(18.8)$ & $21,555(13.9)$ & $2,431(14.9)$ & $19,124(13.8)$ & $18,801(31.2)$ & $2,271(29.8)$ & $16,530(31.4)$ \\
\hline II & $23,202(10.8)$ & $14,622(9.5)$ & $1,666(10.2)$ & $12,956(9.4)$ & $8,580(14.2)$ & $1,136(14.9)$ & $7,444(14.1)$ \\
\hline III & $28,895(13.5)$ & $19,498(12.6)$ & $2,208(13.5)$ & $17,290(12.5)$ & $9,397(15.6)$ & $1,332(17.5)$ & $8,065(15.3)$ \\
\hline Missing & $40,938(19.1)$ & $33,023(21.4)$ & $2,868(17.5)$ & $30,155(21.8)$ & $7,915(13.1)$ & $850(11.2)$ & $7,065(13.4)$ \\
\hline \multicolumn{8}{|l|}{ Primary Site } \\
\hline Oral Cavity & $55,620(25.9)$ & $55,620(36)$ & $6,028(36.8)$ & $49,592(35.9)$ & - & - & - \\
\hline Oropharynx & $81,174(37.8)$ & $81,174(52.5)$ & $8,551(52.2)$ & $72,623(52.6)$ & - & - & - \\
\hline Other Pharynx & $17,731(8.3)$ & $17,731(11.5)$ & $1,787(10.9)$ & $15,944(11.5)$ & - & - & - \\
\hline Larynx & $60,296(28.1)$ & - & - & - & $60,296(100)$ & $7,614(100)$ & $52,682(100)$ \\
\hline \multicolumn{8}{|l|}{ ARC Economy FY2017 } \\
\hline Distressed & $1,842(0.9)$ & $1,112(0.7)$ & $1,112(6.8)$ & - & $730(1.2)$ & $730(9.6)$ & - \\
\hline At-Risk & 3,491 (1.6) & 2,303 (1.5) & $2,303(14.1)$ & - & $1,188(2)$ & $1,188(15.6)$ & - \\
\hline Transitional & $15,935(7.4)$ & $11,022(7.1)$ & $11,022(67.3)$ & - & $4,913(8.1)$ & $4,913(64.5)$ & - \\
\hline Competitive & $2,402(1.1)$ & $1,701(1.1)$ & $1,701(10.4)$ & - & $701(1.2)$ & $701(9.2)$ & - \\
\hline Attainment & $310(0.1)$ & $228(0.1)$ & $228(1.4)$ & - & $82(0.1)$ & $82(1.1)$ & - \\
\hline Not Applicable & $190,841(88.8)$ & $138,159(89.4)$ & - & $138,159(100)$ & $52,682(87.4)$ & - & $52,682(100)$ \\
\hline
\end{tabular}

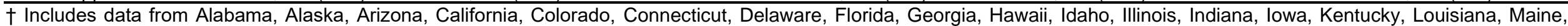

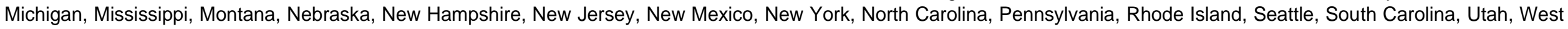
Virginia, Wisconsin, and Wyoming.

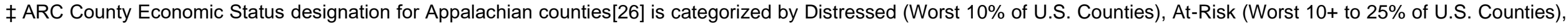

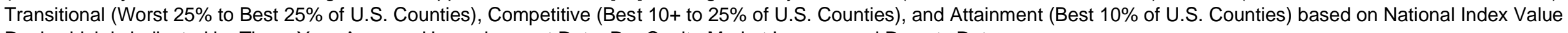
Rank which is indicated by Three-Year Average Unemployment Rate, Per Capita Market Income, and Poverty Rate.

SD: standard deviation; SEM: standard error of the mean

Table 1. Appalachian and Non-Appalachian OC/P and Laryngeal Patient Characteristics 2007-2013 


\section{Study 1 Supplemental Materials: Disparate Survival of Late-stage Male Oropharyngeal Cancer in Appalachia}

Brenen W. Papenberg ${ }^{1}$, Jessica L. Allen ${ }^{1}$, Steven M. Markwell ${ }^{1}$, Erik T. Interval ${ }^{2}$, Phillip A. Montague ${ }^{2}$, Christopher J. Johnson ${ }^{3}$ and Scott A. Weed ${ }^{1 *}$

a

\begin{tabular}{|c|c|c|}
\hline $\begin{array}{c}\text { NAACCR Cohort: } \\
\text { 57,805 Laryngeal } \\
\text { Patients } \\
\text { Appalachian residence } \\
\text { Relative Survival }\end{array}$ & $\rightarrow$ & $\begin{array}{l}\text { Stratify by AJCC-6 } \\
\text { Stage Group I - IV } \\
\text { Stage IV selected } \\
\text { due to lowest } \\
\text { Relative Survival }\end{array}$ \\
\hline
\end{tabular}

b

All Sexes, All Races, All Stages

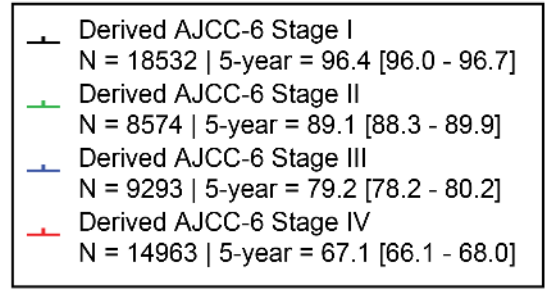

At 60 Months:

- $\mathrm{p}<0.00001-\mathrm{p}<0.00001$

$=0.00001$

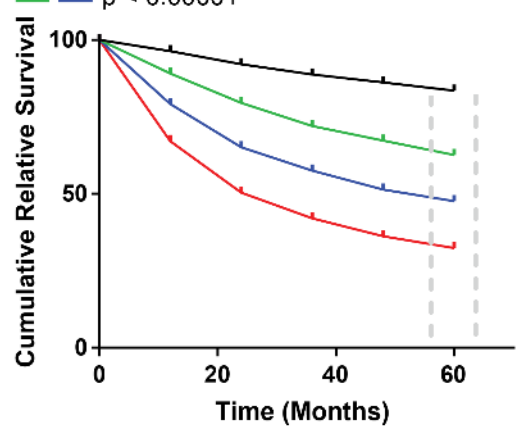

C

\begin{tabular}{|c|c|}
\hline+ & $\begin{array}{l}\text { Non-Appalachian White Males } \\
N=7865 \mid 5 \text {-year }=32.9[31.2-34.6] \\
\text { Appalachian White Males } \\
N=1382 \mid 5 \text {-year }=30.9[26.6-35.2]\end{array}$ \\
\hline & $\begin{array}{l}\text { Non-Appalachian White Females } \\
N=2182 \mid 5 \text {-year }=35.9[32.8-38.9] \\
\text { Appalachian White Females } \\
N=378 \mid 5 \text {-year }=32.9[26.3-39.7]\end{array}$ \\
\hline
\end{tabular}

At 60 Months:

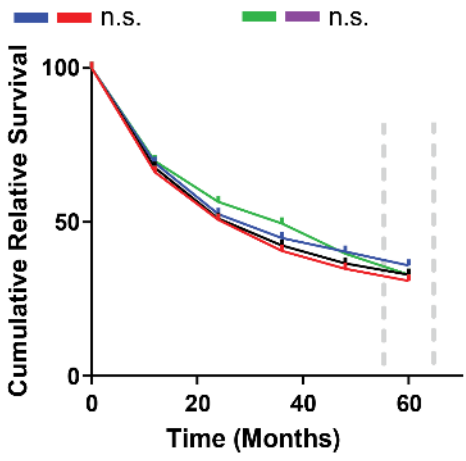

Stratify by race, sex, and

Appalachian residence

No observed Appalachian survival Disparity
Exclude Laryngeal Patients from Study

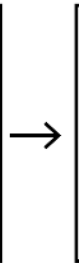

d

\begin{tabular}{|c|c|}
\hline+ & $\begin{array}{l}\text { Non-Appalachian Black Males } \\
\mathrm{N}=2010 \mid \text { 4-year }=30.7[27.3-34.1] \\
\text { Appalachian Black Males } \\
\mathrm{N}=157 \mid \text { 4-year }=24.9[17.0-33.6]\end{array}$ \\
\hline+ & $\begin{array}{l}\text { Non-Appalachian Black Females } \\
\mathrm{N}=551 \mid \text { 4-year }=33.2[23.4-35.1] \\
\text { Appalachian Black Females } \\
\mathrm{N}=32 \mid \text { 4-year }=\mathrm{NA}\end{array}$ \\
\hline
\end{tabular}

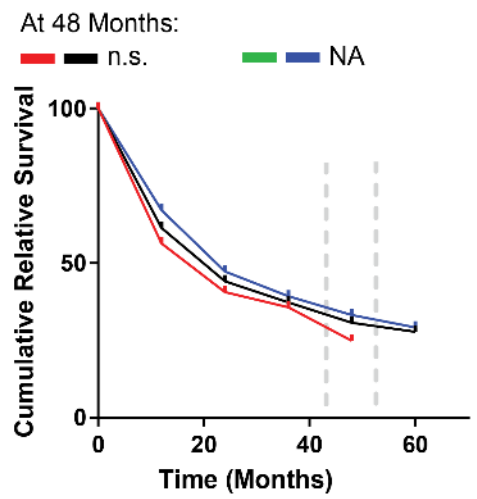

Supplementary Figure S1. Survival analysis of Appalachian Stage IV laryngeal cancer. A. Flow diagram of procedures used to evaluate Appalachian-specific laryngeal cancer survival data. Boxes contain sequential operational steps utilized in the stratification and cohort analysis, noting no difference in survival within the cohort. B. Survival analysis of Appalachian laryngeal cancer stratified by clinical stage. RS values are plotted for each year after diagnosis, with five-year (60 month) ratios evaluated across all AJCC-6 stages. C and D. Survival analysis of white (C) and black (D) stage IV laryngeal cancer stratified by Appalachian residency and sex. RS values are plotted as in (B). P-values between significant groups are shown at the top of each graph; n.s., not significant; NA, not available. Black patients were evaluated for significance at 48 months due to incomplete male survival at 60 months. 
a

\begin{tabular}{|c|c|}
\hline - & $\begin{array}{l}\text { Non-Appalachian States } \\
N=28143 \mid 5 \text {-year }=49.6[48.5-50.7] \\
\text { Georgia Appalachian Counties } \\
N=667 \mid 5 \text {-year }=46.6[37.8-54.9] \\
\text { New York Appalachian Counties } \\
N=280 \mid 5 \text {-year }=45.6[38.1-52.8] \\
\text { North Carolina Appalachian Counties } \\
N=518 \mid 5 \text {-year }=51.1[40.8-60.5] \\
\text { South Carolina Appalachian Counties } \\
N=269 \mid 5 \text {-year }=52.1[37.0-64.8]\end{array}$ \\
\hline
\end{tabular}

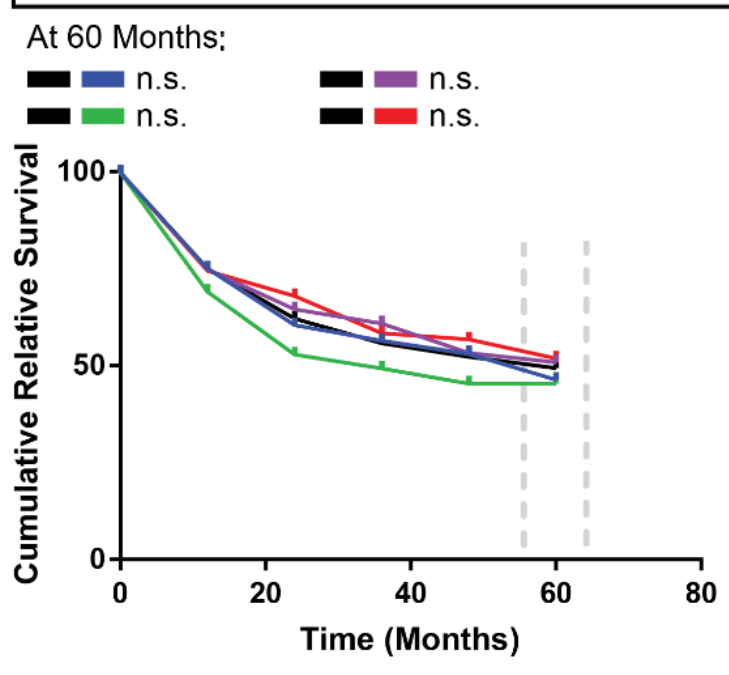

b

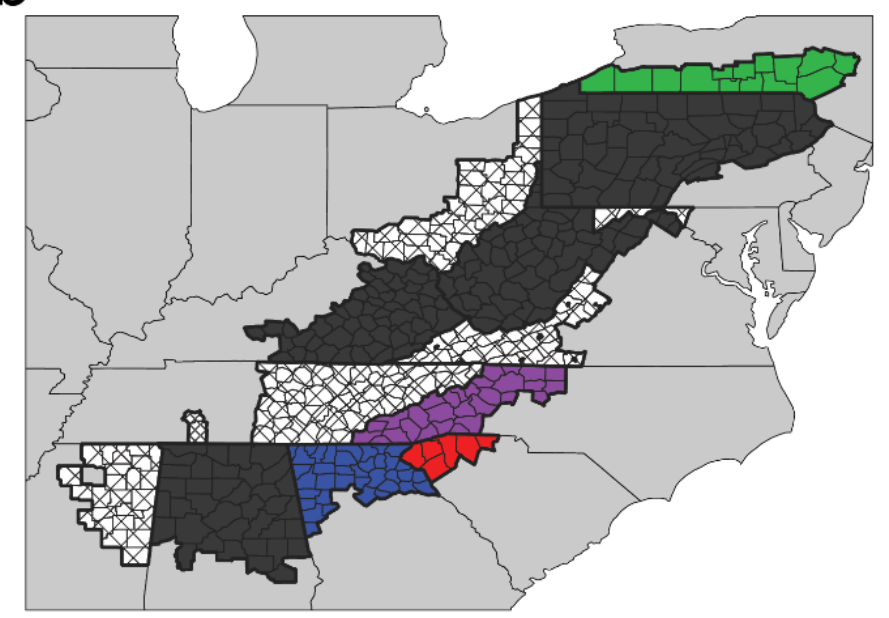

Non-Significant Appalachian States

Significant Appalachian States

Data Not Available

\section{Supplementary Figure S2. Survival analysis of Appalachian states with non-significant OC/P survival. A.}

States lacking disparate RS of stage IV white Appalachian male OC/P. Plotted RS values over time are shown for non-Appalachian states (black), and Appalachian states with non-significant (n.s.) survival. Patient N, fiveyear CRS with $95 \% \mathrm{Cl}$ and $\mathrm{P}$-values at 60 months for each significant Appalachian state are shown at the top of the graph. B. Map of Appalachian region displaying states with non-significant stage IV white Appalachian male OC/P survival. Colored states are non-significant, and significant states are in black; states with no available data are marked with a cross-hatched pattern. 
a

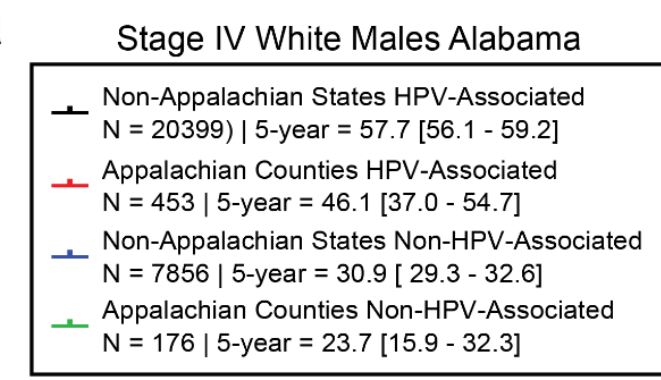

At 60 Months:

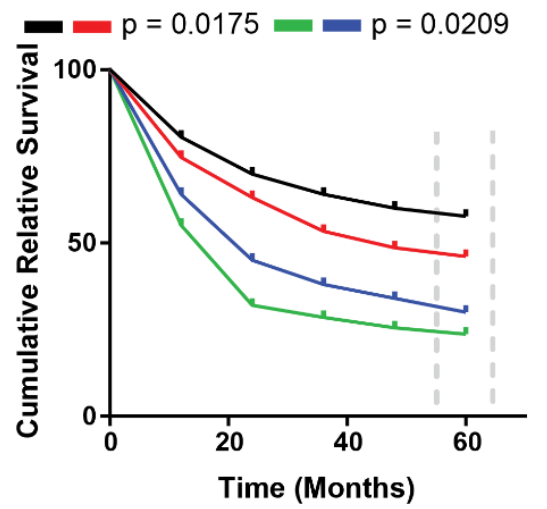

C

Stage IV White Males Pennsylvania

_. Non-Appalachian States HPV-Associated

$\mathrm{N}=20399$ | 5-year $=57.7[56.1-59.2]$

Appalachian Counties HPV-Associated

$N=1104$ | 5-year = 52.0 [44.0 - 59.5]

Non-Appalachian States Non-HPV-Associated

$\mathrm{N}=7856$ | 5-year $=30.9$ [ $29.3-32.6]$

Appalachian Counties Non-HPV-Associated

$\mathrm{N}=594 \mid$ 5-year $=31.0[25.2-36.9]$

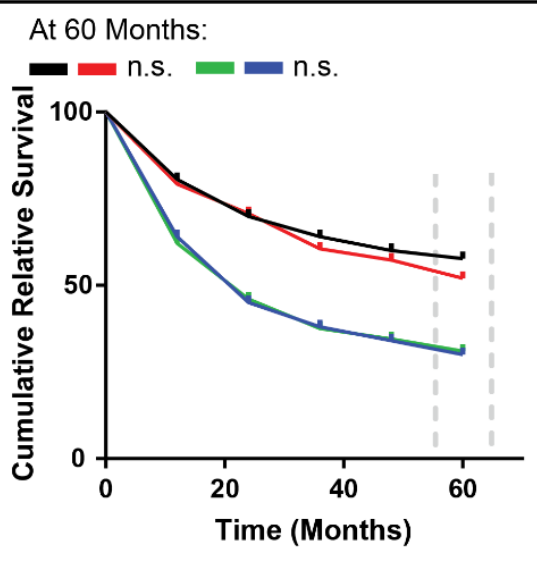

b

\section{Stage IV White Males Kentucky}

\begin{tabular}{|c|c|}
\hline & $\begin{array}{l}\text { Non-Appalachian States HPV-Associated } \\
\mathrm{N}=20399 \mid \text { 5-year }=57.7[56.1-59.2]\end{array}$ \\
\hline- & $\begin{array}{l}\text { Appalachian Counties HPV-Associated } \\
\mathrm{N}=250 \mid 5 \text {-year }=53.9[45.1-61.7]\end{array}$ \\
\hline+ & $\begin{array}{l}\text { Non-Appalachian States Non-HPV-Associated } \\
\mathrm{N}=7856 \mid 5 \text {-year }=30.9[29.3-32.6]\end{array}$ \\
\hline - & $\begin{array}{l}\text { Appalachian Counties Non-HPV-Associated } \\
\mathrm{N}=123 \mid 5 \text {-year }=23.2[15.1-32.4]\end{array}$ \\
\hline
\end{tabular}

At 60 Months:

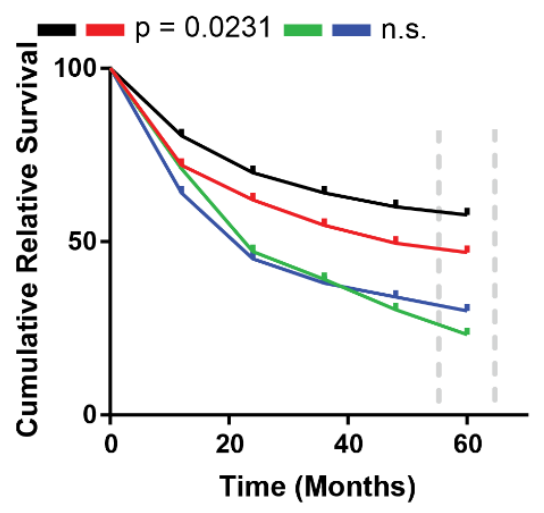

d

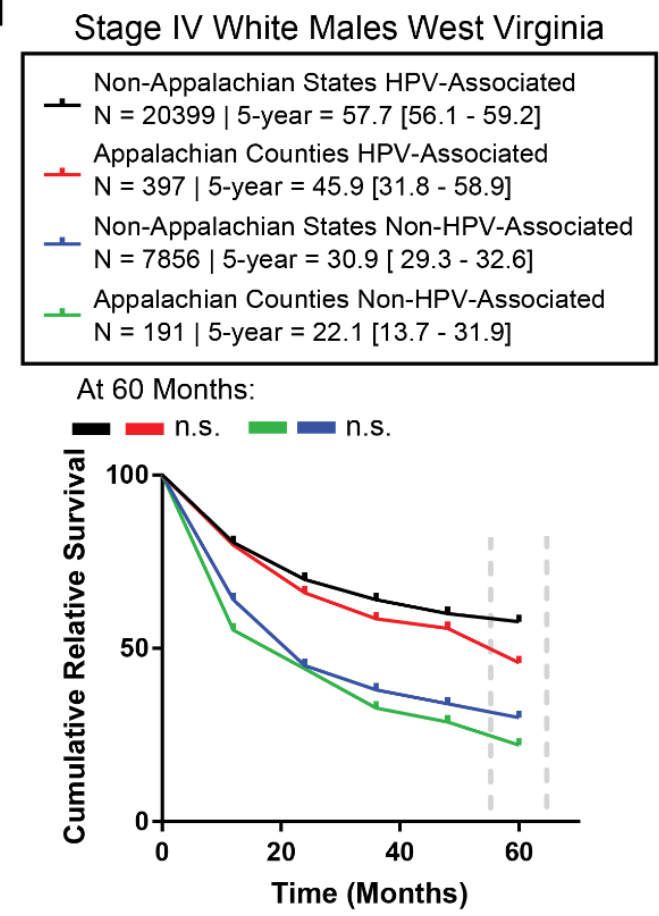

\section{Supplementary Figure S3. Survival analysis of Appalachian states with non-significant OC/P survival. A-}

D. Identification of states with disparate stage IV white Appalachian male HPV-associated oropharyngeal cancer

survival. RS values were plotted for patients stratified as in Figure 3D for each OC/P disparity state. A, Alabama;

B, Kentucky; C, Pennsylvania; D, West Virginia. Patient N, five-year CRS with 95\% Cl and P-values at 60 months for each Appalachian state are shown at the top of the graph; n.s., not significant. 


\section{Study 2: Copy Number Alterations Identify a Smoking-Associated Expression Signature Predictive of Poor Outcome in Head and Neck Squamous Cell Carcinoma}

Brenen W. Papenberg ${ }^{1}$, James Ingles ${ }^{2}$, Si Gao ${ }^{2}$, Jun Feng ${ }^{3}$, Jessica L. Allen ${ }^{1}$, Steven M. Markwell ${ }^{1}$, Erik T. Interval $^{4}$, Phillip A. Montague ${ }^{4}$, Sijin Wen ${ }^{2}$ and Scott A. Weed ${ }^{1^{*}}$

${ }^{1}$ Department of Biochemistry, Program in Cancer Cell Biology, ${ }^{2}$ Department of Biostatistics, ${ }^{3}$ Department of Statistics, ${ }^{4}$ Department of Otolaryngology, Head and Neck Surgery, West Virginia University, Morgantown, West Virginia, 26506

Running title: Smoking-associated genes in head and neck cancer

Corresponding Author: *Scott A. Weed, West Virginia University Cancer Institute, P.O. Box 9300, Morgantown, WV 26506, Phone: 304-293-3016, Fax: 304-293-4667, scweed@hsc.wvu.edu.

Competing Interest Statement: The authors declare no competing financial interests in relation to the work described. 


\section{Abstract}

Cigarette smoking is a risk factor for the development of head and neck squamous cell carcinoma (HNSCC), partially due to tobacco-induced large-scale chromosomal copy-number alterations (CNAs). Identifying CNAs caused by smoking is essential in determining how gene expression from such regions impact tumor progression and patient outcome. We utilized The Cancer Genome Atlas (TCGA) whole genome sequencing data for HNSCC to directly identify amplified or deleted genes correlating with smoking pack-year based on linear modeling. Internal cross-validation identified 35 CNAs that significantly correlated with patient smoking, independent of human papillomavirus (HPV) status. The most abundant CNAs were chromosome 11q13.3-q14.4 amplification and 9p23.1/9p24.1 deletion. Evaluation of patient amplicons reveals four different patterns of 11q13 gene amplification in HNSCC resulting from breakage-fusion-bridge (BFB) events responsible for SAES amplification. Predictive modeling identified 16 genes from these regions that denote poorer overall and disease-free survival with increased pack-year use, constituting a smoking-associated expression signature (SAES). Patients with altered expression of signature genes have increased risk of death and enhanced cervical lymph node involvement. The identified SAES can be utilized as a novel predictor of increased disease aggressiveness and poor outcome in smoking-associated HNSCC. 


\section{Introduction}

HNSCC is an aggressive neoplasm of the upper aerodigestive tract caused by exposure to tobacco, alcohol, and high-risk HPV infection(1). Over 53000 new cases and 11000 deaths are predicted to occur in the U.S. due to HNSCC in 2020(2) with five-year post-treatment survival near 50\%(3). Cervical nodal metastasis is frequently found in HNSCC, and is an important prognostic indicator associated with late stage (American Joint Committee on Cancer (AJCC) stage III/IV) disease in aggressive smoking-associated HPV-negative cancers $(4,5)$.

Widespread next-generation sequencing of HNSCC cohorts has identified driver mutations, common tumor suppressor/oncogenes, and molecular subtypes(6-9). Conclusions from this work indicate that HPV-positive tumors harbor fewer mutations and CNAs than HPV-negative cancer. This is congruent with HPV-positive patients exhibiting better overall survival, stratifying the disease into distinct prognostic subtypes $(3,10)$. Over $50 \%$ of HPV-negative patients present with stage IV disease, where tobacco-generated tumors contain high genomic instability, increased lymphovascular invasion, enhanced therapeutic resistance, poor prognosis and decreased time of survival $(6,7,11-13)$.

CNAs involved in HPV-negative HNSCC progression include gene gains (two extra copies), amplifications (more than two extra copies), loss of heterozygosity, and homozygous deletion of chromosomal segments $(6,9,10,14)$. Widespread loss of chromosomal segments, along with concurrent frequency of mutations in tumor suppressor genes, supports the generalized concept that HNSCC is largely driven by genomic relief of tumor suppression(15). In addition, HPV-negative HNSCC is driven by overexpression of oncogenes in genomically amplified regions $(16,17)$. Large-scale CNAs primarily arise through double-strand DNA breakage at fragile sites and subsequent telomeric loss(18). This initiates a BFB cycle through chromatid annealing, leading to uneven separation during mitosis and increased chromosome segment copy numbers(19).

While smoking-driven genomic damage is well known in $\operatorname{HNSCC}(10,20)$, the precise CNAs correlated with smoking have not been previously defined. Here, we utilized an unbiased, internally-validated statistical approach with the TCGA-HNSC cohort to identify chromosomal homozygous deletions and amplifications in HNSCC that correlated with patient smoking. Currently, this is the only publicly available HNSCC cohort that contains necessary molecular and clinical parameters for patients suitable for such evaluation. Within this cohort, amplification of genes within chromosome 11q13.3-q13.4 and homozygous deletions of genes within 
chromosome 9p21.3/9p24.1 were the only CNAs that correlated significantly with cigarette pack-year usage in an appreciable percentage of patients. A gene subset with altered transcript expression from these regions

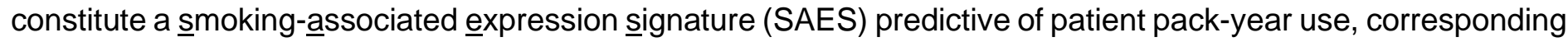
with elevated risk of death and enhanced regional lymph node metastasis. Alignment-based mapping of patient 11q13.3-q13.4 amplicons identifies underlying BFB scenarios responsible for amplified SAES genes. Collectively these results identify CNAs and corresponding genes in HNSCC that correlate positively with cigarette use, confirming known and identifying new oncogenic drivers that may serve as novel biomarkers and targets for improved clinical intervention in patients with documented smoking histories. 


\section{Materials and Methods}

\section{Samples and Clinical Data}

Data were obtained from the 2016_01_28 TCGA-HNSC cohort, downloaded from Broad Genomic Data Analysis Center (GDAC) Firehose(21), consisting of 529 patients with tumors of the oral cavity ( $n=320 / 529,60.5 \%$ ), pharynx ( $n=92 / 529,17.4 \%)$, and larynx $(n=117 / 529,22.1 \%)$. The majority of patients were male $(n=386 / 529$, $73 \%)$ and smoked $(n=299 / 529,56.5 \%)$, with mean 32.4 pack-years exposure. Patients were diagnosed from 1992-2013 with a mean age of 60.7 years. 20 patients were excluded that lacked copy-number or smoking history. Clinical data were retrieved from the level 4 TCGA data file All_CDEs.txt and cBioPortal(22). Alcohol use was calculated by multiplying Alcohol Frequency and Daily Drinks. Patients with no pack-year data were excluded from pack-year correlations. Statistical significance was determined using chi-square test and Bonferroni correction or one-way ANOVA and Tukey's post-hoc with an alpha of 0.05 .

\section{Determination of Smoking-Associated CNAs}

Smoking-associated CNAs were determined by Genomic Identification of Significant Targets in Cancer (GISTIC)2(23) analysis of TCGA 2016_01_28 level 4 data for 24,776 genes(21). Briefly, raw GISTIC2 values within all_data_by_genex.txt were used for analysis. A linear regression model was used to assess the association between gene GISTIC2 value as a dependent variable and smoking in pack-years as an independent variable, adjusting for age, sex, and disease stage. For multiple testing, $p$-value sets were modeled using a betauniform mixture model(24), permitting selection of $p$-value cutoffs bracketing the false discovery rate (FDR). Genes that reached statistical significance (100 maximum) in each analysis, or the top 100 genes if significance was not reached, were analyzed further. Prediction modeling was validated using multiple methods. First, the dataset was randomly separated into training and validation sets, each containing $50 \%$ of patients. Second, the Leave-One-Out cross validation (LOOCV) method was used(25). For each gene in each method, a linear regression model was fit using pack-year value as the explanatory variable, and gene GISTIC2 value as the response using half of all samples (50\%), or all but one sample (LOOCV). The fitted models were used to examine the accuracy of the remaining samples. Predictive capabilities were evaluated through concordance, sensitivity, and specificity after performed for each gene. Third, the "stability selection" method was used to assess the prediction for each gene, which combines the techniques of sub-sampling and randomized Lasso 
algorithms(26). Validated genes were combined by cytogenetic sub-sub band (cytoband). Cytobands altered in fewer than $10 \%$ of patients and those dependent on HPV-status were excluded. Predicted CNA probabilities were graphed based on the fitted model, with data sorted by ascending pack-year. In the time-to-event analysis, survival functions by amplified genes (yes/no) were assessed by Kaplan-Meier and log-rank testing for overall and progression-free survival.

\section{Evaluation of Common Mutations}

Patient individual gene mutations and Total Mutations were obtained from TCGA-HNSC 2016_01_28 level 3 Mutation Packager Raw Calls and the cBioPortal "Mutated Genes" analysis(21,22). Data were stratified by smoking and CNA status. Smoking status was divided into "Never Smoker" (pack-year=0), "Ever Smoker" (packyear>0), "Heavy Smoker" (pack-year>10), "Light Smoker" (0<pack-year $\leq 10)$ and "Low Risk" (pack-year $\leq 10)$, similar to other reports(27).

\section{Determination of the Smoking-associated Expression Signature}

Expression levels of genes identified from the CNA analysis were determined using the TCGA-HNSC 2016_01_28 level 3 RNAseq gene expression dataset(21). Analysis was conducted using z-scores calculated from reads per kilobase of transcript, per million reads (RPKM) in normal adjacent tissues as baseline. Gene expression for overall survival was assessed by comparing the first quartile (lowest $25 \%$ ) of expression to the fourth quartile (highest 25\%) of expression levels. Genes where differential expression significantly affected overall survival were combined to create the SAES. A linear regression model was used to assess the association between the z-score as a dependent variable and smoking pack-year as an independent variable. LOOCV validation was performed as described. The predicted SAES gene expression probability is based on the fitted model, with patients sorted by ascending pack-year. For correlative analysis between lymph node (yes/no) and gene overexpression (z-score $>2$ ) or underexpression (z-score $<-2)$, odds ratios and the $95 \%$ confidence intervals $(\mathrm{Cl})$ for each gene was estimated from a fitted logistic model. Time-to-event survival functions were assessed using the Kaplan-Meier and log-rank testing for overall and progression-free survival (yes/no for overexpression). Cox models were used to assess the association between SAES genes and survival. Estimated relative risk of death was presented graphically based on the fitted model where the data were sorted by standardized SAES expression. 


\section{Amplicon Alignment and Segment Determination}

Thresholded GISTIC2.0 CNA values were downloaded from the GDAC Firehose(21). Values were sorted and aligned in relation to the total length of the entire 11 q13 chromosomal segment and flanking regions. Amplicon segments were derived from previous mapping studies(28-31), aided by natural breaks in the alignment data across cohort patients with 11q13 amplified genes. SAES gene positions were aligned using the University of California Santa Cruz (UCSC) Genome Browser with human genome assembly GRCh38/hg38(32). 


\section{Results}

\section{Cigarette Smoking Correlates with Specific Copy Number Alterations In HNSCC}

To identify smoking-associated CNAs in HNSCC, CNAs containing amplified and deleted genes were extracted and independently evaluated for correlation with pack-year (Fig. 1A) (Supplementary Table S2). 1775 amplified genes were identified that correlated significantly, mapping to 92 cytobands (Fig. 1A). To assess HPV-status as a potential confounding variable, identified genes were correlated with confirmed HPV-status. None of the 92 amplified cytobands exclusively correlated with HPV-positive tumors, whereas 17 cytobands showed selective correlation to HPV-negative tumors. The remaining two cytobands were amplified independent of HPV-status and further evaluated (Fig. 1B). As expected, smokers were more commonly HPV-negative than non-smokers (Supplementary Table S1).

Of the amplified genes that correlated with pack-year, genes located on chromosome 11q13.3 ( $n=20)$ and $11 q 13.4(n=10)$ were from the only cytobands amplified independent of HPV status and in at least $10 \%$ of patients with pack-year data (Fig. 1C). This region contains the chromosomal fragile sites (CFSs) FRA11H and FRA11F and is flanked by two segmental duplications (SDs)(28-31,33-35). 11q13.3 amplification is significantly enriched in patients that smoked (85.6\%) compared to non-smokers (65.1\%) (Fig. 1D). Patients with any 11 q13.3 gene amplification had a 36.3-month median overall survival and a 38.4\% five-year overall survival rate, whereas nonamplified patients had a 65.7-month median overall survival and a 51.8\% five-year overall survival rate (Fig. 1E). Disease-free survival for 11q13.3 amplified patients trended lower but failed to reach significance (Fig. 1F). Similarly, 11q13.4 amplified patients were significantly enriched for smokers (87.5\%) compared to non-smokers (66.2\%) (Fig. 1G). Patients with 11q13.4 gene amplification showed lower overall and progression-free survival, with a 26.8-month median overall survival, and 34.0\% five-year overall survival rate. Non-amplified patients had a 65.7-month median overall survival and 50.4\% five-year overall survival rate (Fig. $1 \mathrm{H}$ ). Five-year disease-free survival for 11 q13.4 amplified individuals was $38.4 \%$ compared with $56.5 \%$ in non-amplified cases (Fig. 1 I). The majority of patients with 11q13.3 gene amplification also had 11q13.4 gene amplification (73.4\%), consistent with the cytobands being contiguous. Due to lack of suitable external validation sets, multiple internal validation methods were used. Separating the dataset into training and validation sets for analysis demonstrated a high specificity (>95\%) and concordance (>73.8\%). LOOCV analysis on each significant gene also demonstrated 
high specificity (>97.4\%) and concordance (>74\%). This unbiased approach independently confirms that amplification of genes within chromosome 11q13.3-q13.4 correspond with this region being the most frequent CNA in HNSCC $(6,16)$, and that 11q13.3-q13.4 gene amplification correlates with cigarette use and poor HNSCC outcome as suggested in previous work $(6,16)$.

In addition, our analysis identified five cytobands that correlated with homozygous deletion and pack-year (Fig. 2A). After evaluating by HPV-status as in Figure 1B, five genes from these cytobands were found to be deleted independent of HPV (Fig. 2B). One gene mapped to cytoband 9p24.1 and four genes mapped to cytoband 9p21.3 (Fig. 2C). This region contains an unnamed CFS (9CFS) and the CFS FRA9C, promoting chromosome breakage and gene deletion(36). Deletion of 9p21.3 was enriched for smokers (80.4\%) compared to nonsmokers (66.2\%) (Fig. 2D). Patients with any 9p21.3 gene deletion had a 35.9-month median overall survival and a $38.5 \%$ five-year overall survival rate, whereas non-deleted patients had a 66.7 -month median overall survival and a 51.2\% five-year overall survival rate (Fig. 2E). Disease-free survival for 9p21.3 deleted patients failed to reach significance (Fig. 2F). Deletion of cytoband 9p24.1 was not enriched for smokers (Fig. 2G) and no difference in overall (Fig. 2H) or disease-free survival (Fig. 2I) was observed. Training and validation analysis demonstrated high specificity (>98\%) and concordance (>69.5\%).

\section{The Majority of Smoking-associated Amplified Genes are Contained Within the Most Frequently Amplified 11q13 Segment in HNSCC.}

The worse outcomes and higher clinical risk parameters associated with genes in 11q13.3.-11q13.4, combined with the variation in amplicon lengths led us to determine the underlying 11 q13 amplification mechanisms. $87 \%$ of 11 q13 amplified patients had distal 11q loss, suggesting BFB as the predominant amplification mechanism. Chromosomal alignment and clustering of all 11q13.3-q13.4-amplified patients from the TCGA-HNSC cohort identified three distinct alignment patterns of amplified regions, grouped as segments 1, 2 and 3 (Fig. 3A). All or part of the segment 2 region is present in every patient with 11q13.3-q13.4 amplification. Based on segment distribution and the FRA11H and FRA11F, four possible BFB scenarios account for all observed $11 \mathrm{q} 13$ amplification patterns in HNSCC (Fig. 3B). Scenario 1 occurs in 17\% of patients, where the first break occurs at the telomeric end of FRA11H before Segment 3 to delete this region. This results in sister chromatid end fusion at segment 2. A subsequent second break at FRA11H between segment 1 and segment 2 results in inverted segment 1 loss, repeated through subsequent rounds of BFB to increase amplicon number (Fig. 3). Additional 
scenarios (2-4) involving breakage at different FRA11H sites, FRA11F, or other combinations also occur at lower frequencies than scenario 1 (Fig. 3B). 13\% of amplified patients have intact 11q telomeres, indicating that SDmediated amplification occurs at lower frequency. Regardless, the resulting segment 2 region in all amplification events contains the 11q13.4-q13.4 chromosomal region that harbors the majority of identified smokingassociated genes (Fig. 1C).

\section{Smoking-associated Copy-number Alteration Corresponds with Elevated Risk Factor Exposure}

Patients with smoking-associated CNAs stratified by cytoband and smoking status were evaluated for clinical characteristics and additional HNSCC risk factors across the cohort (Supplementary Table S1). All patients with 11q13.3-q13.4 amplification (Total Amplified) had significantly higher mean pack-year usage than patients lacking amplification (Total Non-Amplified) (46.6 vs. 27.1). The majority of 11q13.3-q13.4 Amplified Smokers were male with stage IV disease, had positive lymph node involvement, higher alcohol consumption, higher pack-year and were HPV-negative. In addition, the majority of 9p21.3/9p24.1 Deleted Smokers were male with stage IV disease, had positive lymph node involvement, and were HPV-negative.

\section{Differences in the Mutational Profile of Commonly Altered HNSCC Driver Genes in Smoking-associated Copy-number Altered HNSCC}

Tobacco-associated HNSCC demonstrates characteristic mutational signatures that result in oncogene activation or loss of tumor suppression(6,37). Since 11q13.3-q13.4 amplification and 9p21.3 deletion occurs in a greater percentage of cigarette-smoking patients, we examined the mutational occurrence of the most frequently altered HNSCC genes in patients that smoked and contained these CNAs (Table 1). The top ten most commonly mutated cancer-related HNSCC genes were evaluated(6). When stratified by smoking status, the mutation rates of $L R P 1 B(19.6 \%$ smokers; $8.2 \%$ non-smokers), NSD1 (14.2\% smokers; $4.1 \%$ non-smokers) and CASP8 (8.3\% smokers; 16.4\% non-smokers) differed significantly. Smoking was also associated with 11q13.3, 11q13.4 and 9p21.3 gene CNA. Interestingly, the mutation rate difference of TP53, the most commonly mutated gene in $\operatorname{HNSCC}(6,9)$, did not differ significantly by smoking status (71.1\% smokers; $63.9 \%$ non-smokers) when defining smokers as patients with an above zero pack-year or with a reported smoking history (Table 1, Supplementary Table S3). When patients were stratified by 11q13.3-q13.4 amplification, the mutation rates of CASP8 (2.2\% amplified; 13.1\% non-amplified), KMT2D (0.7\% amplified; 15\% non-amplified), NOTCH1 (16.2\% 
amplified; 17.4\% non-amplified), NSD1 (6.7\% amplified; 13.7\% non-amplified), PCLO (19.1\% amplified; $14.2 \%$ non-amplified), and TP53 (83.8\% amplified; 64.1\% non-amplified) were significantly different. 11q13.3-q13.4 amplification was also significantly associated with $9 p 21.3$ deletion. When patients were stratified by 9p21.3/9p24.1 deletion, the mutation rates of NOTCH1 (23\% deleted; $13.5 \%$ non-deleted) and TP53 (76.4\% deleted; 65.1\% non-deleted) were significantly different. 9p21.3/9p24.1 deletion was also significantly associated with 11q13.3-q13.4 amplification. CDKN2A mutation rate was also significantly different (8.9\% deleted; $29.2 \%$ non-deleted); however, CDKN2A is located in the deleted 9p21.3 region. All other evaluated gene mutation rates were not significantly different across the cohort, regardless of smoking or CNA status.

\section{Identification of the Smoking-associated Expression Signature in HNSCC Patients with Smoking-} associated CNAs

To determine the expression of actionable drivers in smoking-associated CNAs, mRNA expression was analyzed for each cross-validated gene. Inclusion criteria were restricted to patients with full mRNA sequencing data and RPKM values for each gene, known copy-number status and smoking history. 260 patients contained suitable RNAseq data for analysis (Figure 4A); nine non-protein coding genes in the 11q13.3-q13.4 region (DEFB108B, MIR3164, MIR548K, ENPP7P8, ZNF705E, UNC93B6, FAM86C1, MIR3664, and RNA5SP342) did not have reported RPKM values and could not be analyzed. The remaining genes were assessed for expression differences between patients with CNAs and euploid patients. Patients with CNAs had significantly higher percentages of overexpression or underexpression of most genes (Figure 4B). Genes from this group with expression differences that significantly decreased overall survival were combined to generate a HNSCC CNAbased SAES (Fig. 4C). Ten genes did not alter overall survival based on expression and were summarily excluded (Supplementary Table S4, Supplementary Figure S1). Patients with overexpression or underexpression of one SAES gene demonstrated lower overall survival (Fig. 4D), where patients with overexpression or underexpression of at least three SAES genes also had significantly worse progression-free survival (Fig. 4E). The impact of SAES gene expression on survival was additive, with decreased survival observed in patients with increasing numbers of SAES genes (Fig. 4D and E, Supplementary Figure S2). Multivariate analysis with age, sex, AJCC stage, HPV status, extracapsular spread, perineural invasion, and lymphovascular invasion confirmed overexpression of ANO1, CCND1, CTTN, FADD, MRPL21, and TPCN2 as independent predictors of overall survival in the SAES (Supplementary Table S5). 
Increased Cigarette Smoking Predicts Smoking-associated CNAs and the Smoking Expression Signature

We evaluated patient pack-year data with smoking-associated gene CNA to determine if it could be used to determine CNA risk. Each gene was fit into a linear regression model, with pack-year as the explanatory variable and gene amplification for the response of one testing sample. This was used to determine the probability of CNA for each gene (Supplementary Table S6). Significant genes were combined to create average curves for log odds changes with smoking pack-year and the log odds of gene CNA in non-smokers across all genes, using parameter averages for linear regression models for each gene (Fig. 5A). A parallel analysis was utilized based on the gene RPKM values in the SAES with smoking pack-year to generate regression curves for overexpressed or underexpressed genes mapping to SAES regions (Fig. 5B). Only genes mapping to 11q13.3-q13.4 were significant for both analyses (Supplementary Table S7). Mean gene amplification and overexpression levels were predicted to increase with increasing pack-year value, correlating increased smoking levels with higher risk of 11q13.3-q13.4 amplification and SAES transcript overexpression in HNSCC.

\section{Smoking-associated Expression Signature Genes Predict Increased Risk of Death and Cervical Lymph Node Metastasis}

Since regional cervical lymph node metastasis in HNSCC is the primary clinical parameter for poor clinical outcome $(38,39)$, we determined the relative risk of death and lymph node involvement in HNSCC patients with the SAES. All patients in the TCGA-HNSC cohort were evaluated regardless of smoking status to compensate for the presence of SAES in patients using other tobacco forms that can potentially result in tobaccoassociated CNA(40). For each SAES gene, a positive correlation was observed between risk of death and standardized expression (Fig. 5C, Supplementary Figure S3). Overexpression of an eight-gene SAES subset predicted significantly an increased odds of lymph node metastasis, (Fig. 5D), identifying specific SAES genes that may drive poorer outcomes through increased metastatic risk. 


\section{Discussion}

Genes mapping to the amplified 11q13.3-q13.4 cytobands and the deleted 9p21.3 and 9p24.1 cytobands were the only CNAs that correlated with pack-year in a significant number of HNSCC patients in this cohort. Patients with smoking-associated CNAs have worse overall survival, smoked more, were predominantly male, and HPVnegative. Correlation analysis of transcript expression of smoking-associated CNA genes identified an expression subset associated with poor outcome and nodal involvement. Increased CNA risk and transcript overor underexpression are associated with elevated patient smoking levels, identifying a novel gene group that can be used for improved screening and target investigation for treatment of late stage HNSCC patients with smoking histories.

Correlation of clinical parameters with molecular markers from comprehensive genomic analyses has emerged as a powerful tool for prognostic analysis in cancer medicine $(41,42)$. Integrated analysis of TCGA and other nonpublic HNSCC molecular cohorts have identified genomic and mutational patterns that have been instrumental in determining prognostic aspects of HPV involvement, molecular subtyping and mutational status associated with different HNSCC medical aspects(6,7,43-45). Many CNAs identified in these studies have been previously noted in HNSCC and proposedly due to tobacco-induced genomic instability $(6,16,37)$. In addition, our analysis identified other smoking-associated CNAs that reached significance in an HPV-dependent manner (Figures 1B and 2B). Of these, genes mapping to amplified 3q23-q29 (SOX2/TP63/PIK3CA), deleted 2q22.1 (LRP1B) and 8p23.2 (CSMD1) have been previously noted to be amplified or deleted in $\operatorname{HNSCC}(46-48)$, supporting the utilized correlation methodology.

Although recent sequencing work supports near-mutual exclusion between tobacco-induced, HPV-negative tumors with tobacco-associated CNAs and HPV-positive tumors lacking tobacco-associated CNAs(6,37,49-51), other studies report smoking-associated CNAs present in HPV-positive patients, although at lower levels than HPV-negative cancers(52). HPV-positive patients with smoking histories have progressively worse outcomes corresponding with elevated pack year(53). Such patients would have an increased likelihood of smokingassociated CNAs and thus manifestation of the SAES in HPV-positive tumors, supporting the observed lack of HPV-positivity with smoking and smoking-associated CNAs revealed in this study. 
The tobacco-associated CNAs have been well documented to negatively impact patient prognosis and have been an area of intense study for several decades(54-60). Tobacco-associated CNAs can occur early in malignant development and throughout dysplastic progression to invasive carcinoma(54). Initial TCGA analysis indicated that smoking-associated CNAs corresponded with TP53 mutation and worse outcome in HPV-negative patients(6). Consistent with this, we found higher TP53 mutation and smoking-associated CNA frequency, but note that TP53 mutational status is independent of patient smoking history using a univariate approach (Supplementary Table S1). While this contrasts with previous work linking TP53 mutation with cigarette smoking in HNSCC(61), it is consistent with the TCGA-HNSC Clinical vs. Mutation Level 4 D1V10 analysis, showing no relationship between TP53 status and pack-year(6,21). This may be due to varying cutoffs of pack-year usage (Supplementary Table S3). Regardless, there is clear evidence that smoking leads to TP53 mutation and increases DNA damage through continued cigarette use(62,63). Mutated TP53 unable to properly surveil and/or repair double-strand DNA breaks will lead to increased instability during replicative stress(28,64). Distal 11q loss and deletion of the ATM DNA damage repair gene is frequent in $11 q 13$ amplified HNSCC $(16,18,65)$. Telomeric $11 \mathrm{q}$ loss is also associated with 11q22 co-amplification in 11q13-amplified HNSCC, with cooperativity between genes proposed as a selective pressure for co-amplification(6). We note that co-amplification of these regions in the TCGA cohort occurs at low frequency (3.6\%) and that $11 \mathrm{q} 22$ gene amplification does not correlate with smoking. 11q13/11q22 co-amplification may therefore be driven by other factors that promote DNA breakage near the chromosome 11q telomere.

$11 \mathrm{q} 13$ amplicon sizes vary from $0.65 \mathrm{Mb}$ to over $5 \mathrm{Mb}(34)$. Amplicon alignment allows for identification of conserved amplification patterns, providing insight into smoking-induced genomic damage. Segment alignment with FRA11H and FRA11F indicate that 11q13.3-q13.4 SAES genes cluster within segment 2. This is similar to previous 11 q13 amplicon gene alignments in HNSCC, breast and other cancers(16,66,67). Harmonizing with these designations, the previously established core 1 alignment corresponds with segment 1 , core 2 with segment 2, and cores 3 and 4 with segment 3(67). Over half of 11q13-amplified HNSCC patients exhibit the scenario 1 event involving successive breakage at FRA11F and FRA11H (Fig. 4B). Increased fragile site breakage involving FRA11F and FRA11H in BFB formation has been previously proposed as a tobaccomediated mechanism utilized in 11 q13 amplification $(19,31,68)$, collectively suggesting that progressive breakage at these sites is preferentially favored during the scenario 1 BFB cycle. Irrespective of the amplification scenario, 
segment 2 amplification levels and subsequent SAES gene expression is predicted to increase with elevated smoking rates, consistent with tobacco-induced fragile site involvement in generating SAES gene amplification.

Simultaneous 11q13 amplicon gene overexpression implies cooperativity between gene products impacting multiple cancer hallmarks to maintain and enhance the neoplastic phenotype. Previous work on 11q13 amplicon genes has proposed that the cell cycle regulator CCND1, actin cytoskeletal protein CTTN and apoptosis scaffold FADD function as the primary $11 \mathrm{q} 13$ amplicon drivers in HNSCC, since amplification and overexpression of each of these genes accelerates patient mortality $(54,56)$. These genes are included in the SAES and were validated as independent predictors of overall survival, underscoring their importance in promoting the poor outcomes observed in 11q13-amplified HNSCC cohorts(50,69). Additional amplified SAES genes with known roles in HNSCC include the ORAOV1/LTO1(70), PPFIA1(71), ANO1(72) and FGF19(73). Functions of the remaining SAES genes in HNSCC are largely unknown, warranting future investigation to determine their respective contributions in 11q13-amplified HNSCC.

CCND1 and CTTN amplification and overexpression corresponds with increased lymph node involvement in numerous cohorts, suggesting their use as biomarkers of aggressive disease $(74,75)$. These genes predict increased metastatic lymph node risk (Fig 5D). Importantly, CCND1 had the highest lymph node metastatic odds ratio in our study, congruent with overexpression utilized as a biomarker for increased metastatic risk(76). Apart from CTTN, the remaining SAES with significantly higher lymph node risk remain to be evaluated as independent markers of lymph node involvement and/or investigated as novel targets in aggressive disease. The importance of understanding how 11q13 amplification and resultant gene overexpression contributes to poor outcome in smoking-induced HNSCC is further underscored by increased resistance to chemotherapy and epidermal growth factor receptor targeting in 11q13-amplified HNSCC(77-79). This highlights a persistent need for earlier and precise diagnosis for patients with SAES CNA and expression status to provide improved clinical management of tobacco-positive, HPV-negative HNSCC. 


\section{Acknowledgments}

This work was supported by National Institutes of Health (NIH) pilot grants U54GM104942, P20GM103434 and American Cancer Society (ACS) pilot grant ACS-IRG-16-143-07. The content is solely the responsibility of the authors and does not necessarily represent the official views of the NIH or ACS. Additional support was provided by the West Virginia University Department of Biochemistry, Department of Otolaryngology, and by Transition Grant Support from the Office of Research and Graduate Education. The results published here are in whole or part based upon data generated by the TCGA Research Network: https://www.cancer.gov/tcga.

\section{Competing Interests}

The author(s) declare no competing interests. 


\section{References}

1. Chow LQM. Head and neck cancer. Longo DL, editor. N Engl J Med [Internet]. 2020 Jan 2 [cited 2020 May 4];382(1):60-72. Available from: http://www.nejm.org/doi/10.1056/NEJMra1715715

2. Siegel RL, Miller KD, Jemal A. Cancer statistics, 2020. CA Cancer J Clin [Internet]. 2020 Jan 8 [cited 2020 May 4];70(1):7-30. Available from: https://onlinelibrary.wiley.com/doi/abs/10.3322/caac.21590

3. Argiris A, Karamouzis M V, Raben D, Ferris RL. Head and neck cancer. Lancet (London, England) [Internet]. 2008;371(9625):1695-709. Available from:

http://www.sciencedirect.com/science/article/pii/S014067360860728X

4. Goldson TM, Han Y, Knight KB, Weiss HL, Resto VA. Clinicopathological predictors of lymphatic metastasis in HNSCC: implications for molecular mechanisms of metastatic disease. J Exp Ther Oncol [Internet]. 2010;8(3):211-21. Available from:

http://www.pubmedcentral.nih.gov/articlerender.fcgi?artid=2929983\&tool=pmcentrez\&rendertype=abstra ct

5. Ferlito A, Rinaldo A, Devaney KO, MacLennan K, Myers JN, Petruzzelli GJ, et al. Prognostic significance of microscopic and macroscopic extracapsular spread from metastatic tumor in the cervical lymph nodes. Oral Oncol. 2002;38(8):747-51.

6. Lawrence MS, Sougnez C, Lichtenstein L, Cibulskis K, Lander E, Gabriel SB, et al. Comprehensive genomic characterization of head and neck squamous cell carcinomas. Nature [Internet]. 2015;517(7536):576-82. Available from: http://www.nature.com/doifinder/10.1038/nature14129

7. Keck MK, Zuo Z, Khattri A, Stricker TP, Brown CD, Imanguli M, et al. Integrative Analysis of Head and Neck Cancer Identifies Two Biologically Distinct HPV and Three Non-HPV Subtypes. Clin Cancer Res [Internet]. 2015;21(4):870-81. Available from: http://clincancerres.aacrjournals.org/cgi/doi/10.1158/10780432.CCR-14-2481

8. Maitra A, Biswas NK, Amin K, Kowtal P, Kumar S, Das S, et al. Mutational landscape of gingivo-buccal oral squamous cell carcinoma reveals new recurrently-mutated genes and molecular subgroups. Nat Commun [Internet]. 2013 Dec 2 [cited 2019 Apr 6];4(1):2873. Available from: 
http://www.nature.com/articles/ncomms3873

9. Stransky N, Egloff AM, Tward AD, Kostic AD, Sougnez C, Mckenna A, et al. The Mutational Landscape of Head Squamous Cell Carcinoma. Science (80- ). 2014;333(6046):1157-60.

10. Hayes DN, Van Waes C, Seiwert TY. Genetic landscape of human papillomavirus-associated head and neck cancer and comparison to tobacco-related tumors. J Clin Oncol [Internet]. 2015 Oct 10 [cited 2020 Jun 19];33(29):3227-34. Available from: http://www.ncbi.nlm.nih.gov/pubmed/26351353

11. Hammerman PS, Neil Hayes D, Grandis JR. Therapeutic insights from genomic studies of head and neck squamous cell carcinomas. Cancer Discov. 2015;5(3):239-44.

12. Mroz EA, Tward AD, Pickering CR, Myers JN, Ferris RL, Rocco JW. High intratumor genetic heterogeneity is related to worse outcome in patients with head and neck squamous cell carcinoma. Cancer. 2013;119(16):3034-42.

13. Dillon MT, Harrington KJ. Human papillomavirus-negative pharyngeal cancer. J Clin Oncol. 2015;33(29):3251-61.

14. Noutomi Y, Oga A, Uchida K, Okafuji M, Ita M, Sawauchi S, et al. Comparative genomic hybridization reveals genetic progression of oral squamous cell carcinoma from dysplasia via two different tumourigenic pathways. J Pathol. 2006;210(1):67-74.

15. Birkeland AC, Ludwig ML, Spector ME, Brenner JC. The potential for tumor suppressor gene therapy in head and neck cancer. Discov Med [Internet]. 2016;21(113):41-7. Available from:

http://www.ncbi.nlm.nih.gov/pubmed/26896601\%5Cnhttp://www.pubmedcentral.nih.gov/articlerender.fcg i?artid=PMC4772772

16. Gollin SM. Cytogenetic Alterations and their Molecular Genetic Correlates in Head and Neck Squamous Cell Carcinoma: A Next Generation Window to the Biology of Disease. Genes Chromosomes Cancer [Internet]. 2014 Dec;53(12):972-90. Available from: http://www.ncbi.nlm.nih.gov/pubmed/25183546

17. Alsahafi E, Begg K, Amelio I, Raulf N, Lucarelli P, Sauter T, et al. Clinical update on head and neck cancer: molecular biology and ongoing challenges [Internet]. Vol. 10, Cell Death and Disease. Nature Publishing Group; 2019 [cited 2020 Jun 19]. p. 540. Available from: 
http://www.ncbi.nlm.nih.gov/pubmed/31308358

18. Parikh RA, White JS, Huang X, Schoppy DW, Baysal BE, Baskaran R, et al. Loss of distal 11q is associated with DNA repair deficiency and reduced sensitivity to ionizing radiation in head and neck squamous cell carcinoma. Genes Chromosom Cancer [Internet]. 2007 Aug [cited 2020 Jun 19];46(8):761-75. Available from: http://www.ncbi.nlm.nih.gov/pubmed/17492757

19. Reshmi SC, Roychoudhury S, Yu Z, Feingold E, Potter D, Saunders WS, et al. Inverted duplication pattern in anaphase bridges confirms the breakage-fusion-bridge (BFB) cycle model for 11 q13 amplification. Cytogenet Genome Res. 2007;116(1-2):46-52.

20. Jethwa AR, Khariwala SS. Tobacco-related carcinogenesis in head and neck cancer. Cancer Metastasis Rev. 2017;36(3):411-23.

21. Broad Institute TCGA Genome Data Analysis Center (2016): Firehose 2016_01_28 run. Broad Institute of MIT and Harvard. doi:10.7908/C11G0KM9.

22. Cerami E, Gao J, Dogrusoz U, Gross BE, Sumer SO, Aksoy BA, et al. The cBio Cancer Genomics Portal: An open platform for exploring multidimensional cancer genomics data. Cancer Discov. 2012;2(5):401-4.

23. Mermel CH, Schumacher SE, Hill B, Meyerson ML, Beroukhim R, Getz G. GISTIC2.0 facilitates sensitive and confident localization of the targets of focal somatic copy-number alteration in human cancers. Genome Biol [Internet]. 2011 [cited 2020 Jun 19];12(4):R41. Available from: http://www.ncbi.nlm.nih.gov/pubmed/21527027

24. Pounds S, Morris SW. Estimating the occurrence of false positives and false negatives in microarray studies by approximating and partitioning the empirical distribution of p-values. Bioinformatics. 2003;19(10):1236-42.

25. Refailzadeh Payam, Lei Tang, Huan Liu ASU. Cross-Validaton. Encycl Database Syst. 2009;532-8.

26. Meinshausen N, Peter B. Stability selection (2009-03-16). 2009;1-30.

27. Marur S, Li S, Cmelak AJ, Gillison ML, Zhao WJ, Ferris RL, et al. E1308: Phase II trial of induction 
chemotherapy followed by reduced-dose radiation and weekly cetuximab in patients with HPVassociated resectable squamous cell carcinoma of the oropharynx- ECOG-ACRIN cancer research group. J Clin Oncol [Internet]. 2017 Feb 10 [cited 2020 Jun 19];35(5):490-7. Available from: http://www.ncbi.nlm.nih.gov/pubmed/28029303

28. Coquelle A, Pipiras E, Toledo F, Buttin G, Debatisse M. Expression of fragile sites triggers intrachromosomal mammalian gene amplification and sets boundaries to early amplicons. Cell [Internet]. 1997 [cited 2020 Jul 2];89(2):215-25. Available from: https://pubmed.ncbi.nlm.nih.gov/9108477/

29. Zainabadi K, Jain A V, Donovan FX, Elashoff D, Rao NP, Murty V V, et al. One in four individuals of African-American ancestry harbors a 5.5kb deletion at chromosome 11q13.1. Genomics. 2014;103(4):276-87.

30. Bester AC, Kafri M, Maoz K, Kerem B. Infection with retroviral vectors leads to perturbed DNA replication increasing vector integrations into fragile sites. Sci Rep. 2013;3.

31. Reshmi SC, Huang X, Schoppy DW, Black RC, Saunders WS, Smith DI, et al. Relationship between FRA11F and 11q13 gene amplification in oral cancer. Genes Chromosom Cancer. 2007;46(2):143-54.

32. James Kent W, Sugnet CW, Furey TS, Roskin KM, Pringle TH, Zahler AM, et al. The human genome browser at UCSC. Genome Res. 2002;12(6):996-1006.

33. Zhan T, Fu S, Dong J, Shi Z, Hao J, Zhao Z, et al. Characterization of genetic rearrangements in esophageal squamous carcinoma cell lines by a combination of M-FISH and array-CGH: further confirmation of some split genomic regions in primary tumors. BMC Cancer. 2012;12(1).

34. Gibcus JH, Kok K, Menkema L, Hermsen MA, Mastik M, Kluin PM, et al. High-resolution mapping identifies a commonly amplified 11q13.3 region containing multiple genes flanked by segmental duplications. Hum Genet. 2007;121(2):187-201.

35. Martin CL, Reshmi SC, Ried T, Gottberg W, Wilson JW, Reddy JK, et al. Chromosomal imbalances in oral squamous cell carcinoma: Examination of 31 cell lines and review of the literature. Oral Oncol [Internet]. 2008 Apr [cited 2020 Jun 19];44(4):369-82. Available from:

http://www.ncbi.nlm.nih.gov/pubmed/17681875 
36. Bignell GR, Greenman CD, Davies H, Butler AP, Edkins S, Andrews JM, et al. Signatures of mutation and selection in the cancer genome. Nature [Internet]. 2010 Feb [cited 2020 Jun 19];463(7283):893-8. Available from: http://www.nature.com/articles/nature08768

37. Gillison ML, Akagi K, Xiao W, Jiang B, Pickard RKL, Li J, et al. Human papillomavirus and the landscape of secondary genetic alterations in oral cancers. Genome Res [Internet]. 2019;29(1):1-17. Available from: http://www.ncbi.nlm.nih.gov/pubmed/30563911

38. Marur S, Forastiere AA. Head and Neck Squamous Cell Carcinoma: Update on Epidemiology, Diagnosis, and Treatment. Mayo Clin Proc [Internet]. 2016;91(3):386-96. Available from: http://dx.doi.org/10.1016/j.mayocp.2015.12.017

39. Mermod M, Tolstonog G, Simon C, Monnier Y. Extracapsular spread in head and neck squamous cell carcinoma: A systematic review and meta-analysis. Oral Oncol [Internet]. 2016;62(2016):60-71. Available from: http://dx.doi.org/10.1016/j.oraloncology.2016.10.003

40. Upadhyay P, Gardi N, Desai S, Chandrani P, Joshi A, Dharavath B, et al. Genomic characterization of tobacco/nut chewing HPV-negative early stage tongue tumors identify MMP10 as a candidate to predict metastases. Oral Oncol [Internet]. 2017 [cited 2020 Jun 19];73:56-64. Available from: http://www.ncbi.nlm.nih.gov/pubmed/28939077

41. Mehta S, Lasham A, Blenkiron C, Shelling A, Muthukaruppan A, Laking G, et al. Predictive and prognostic molecular markers for cancer medicine. Ther Adv Med Oncol. 2010;2(2):125-48.

42. Liu ET. Mechanism-derived gene expression signatures and predictive biomarkers in clinical oncology. Proc Natl Acad Sci. 2005;102(10):3531-2.

43. Leemans CR, Snijders PJF, Brakenhoff RH. The molecular landscape of head and neck cancer. Nat Rev Cancer. 2018;18(5):269-82.

44. Huang L, David O, Cabay RJ, Valyi-Nagy K, Macias V, Zhong R, et al. Molecular Classification of Lymph Node Metastases Subtypes Predict for Survival in Head and Neck Cancer. Clin Cancer Res. 2018;25(6):1795-808.

45. Campbell JD, Yau C, Bowlby R, Liu Y, Brennan K, Fan H, et al. Genomic, Pathway Network, and 
Immunologic Features Distinguishing Squamous Carcinomas. Cell Rep. 2018;23(1):194-212.e6.

46. Davidson MA, Shanks EJ. 3q26-29 Amplification in head and neck squamous cell carcinoma: a review of established and prospective oncogenes. FEBS J. 2017;284(17):2705-31.

47. Ma C, Quesnelle KM, Sparano A, Rao S, Park MS, Cohen MA, et al. Characterization CSMD1 in a large set of primary lung, head and neck, breast and skin cancer tissues. Cancer Biol Ther [Internet]. 2009 May [cited 2020 Jun 19];8(10):907-16. Available from: http://www.ncbi.nlm.nih.gov/pubmed/19276661

48. Nakagawa T, Pimkhaokham A, Suzuki E, Omura K, Inazawa J, Imoto I. Genetic or epigenetic silencing of low density lipoprotein receptor-related protein 1B expression in oral squamous cell carcinoma. Cancer Sci. 2006;97(10):1070-4.

49. Cheng H, Yang X, Si H, Saleh AD, Xiao W, Coupar J, et al. Genomic and Transcriptomic Characterization Links Cell Lines with Aggressive Head and Neck Cancers. Cell Rep [Internet]. 2018;25(5):1332-1345.e5. Available from: http://www.ncbi.nlm.nih.gov/pubmed/30380422

50. Hermida-Prado F, Menéndez ST, Albornoz-Afanasiev P, Granda-Diaz R, Álvarez-Teijeiro S, Villaronga MÁ, et al. Distinctive Expression and Amplification of Genes at $11 q 13$ in Relation to HPV Status with Impact on Survival in Head and Neck Cancer Patients. J Clin Med [Internet]. 2018 Dec 1;7(12):501. Available from: http://www.ncbi.nlm.nih.gov/pubmed/30513772

51. Seiwert TY, Zuo Z, Keck MK, Khattri A, Pedamallu CS, Stricker T, et al. Integrative and comparative genomic analysis of HPV-positive and HPV-negative head and neck squamous cell carcinomas. Clin Cancer Res [Internet]. 2015 Feb 1 [cited 2020 Jun 19];21(3):632-41. Available from: http://www.ncbi.nlm.nih.gov/pubmed/25056374

52. Dogan S, Xu B, Middha S, Vanderbilt CM, Bowman AS, Migliacci J, et al. Identification of prognostic molecular biomarkers in $157 \mathrm{HPV}$-positive and HPV-negative squamous cell carcinomas of the oropharynx. Int J Cancer. 2019;145(11):3152-62.

53. Kompelli AR, Morgan P, Li H, Harris W, Day TA, Neskey DM. Prognostic Impact of High-Risk Pathologic Features in HPV-Related Oropharyngeal Squamous Cell Carcinoma and Tobacco Use. Otolaryngol Head Neck Surg (United States). 2019;160(5):855-61. 
54. Ramos-García P, Ruiz-Ávila I, Gil-Montoya JA, Ayén Á, González-Ruiz L, Navarro-Triviño FJ, et al. Relevance of chromosomal band 11q13 in oral carcinogenesis: An update of current knowledge. Oral Oncol. 2017;72(2017):7-16.

55. Noorlag R, van Kempen PMW, Stegeman I, Koole R, van Es RJJ, Willems SM. The diagnostic value of $11 q 13$ amplification and protein expression in the detection of nodal metastasis from oral squamous cell carcinoma: a systematic review and meta-analysis. Virchows Arch. 2015;466(4):363-73.

56. Schuuring E. The involvement of the chromosome 11q13 region in human malignancies : cyclin D1 and EMS1 are two new candidate oncogenes- a review *. 1995;159:83-96.

57. Lammie GA, Fantl V, Smith R, Schuuring E, Brookes S, Michalides R, et al. D11S287, a putative oncogene on chromosome 11q13, is amplified and expressed in squamous cell and mammary carcinomas and linked to BCL-1. Oncogene [Internet]. 1991 Mar 1 [cited 2020 Jun 19];6(3):439-44. Available from: http://www.ncbi.nlm.nih.gov/pubmed/2011398

58. Nobori T, Miura K, Wu DJ, Lois A, Takabayashi K, Carson DA. Deletions of the cyclin-dependent kinase-4 inhibitor gene in multiple human cancers. Nature [Internet]. 1994 Apr [cited 2020 Jun 19];368(6473):753-6. Available from: http://www.nature.com/articles/368753a0

59. Kamb A, Gruis NA, Weaver-Feldhaus J, Liu Q, Harshman K, Tavtigian S V, et al. A cell cycle regulator potentially involved in genesis of many tumor types. Science (80- ) [Internet]. 1994 Apr 15 [cited 2020 Jun 19];264(5157):436-40. Available from: http://www.ncbi.nlm.nih.gov/pubmed/8153634

60. Michael E. Williams, Michael J. Gaffey, Lawrence M. Weiss, Sharon P. Wilczynski, Ed Schuuring PAL, Williams ME, Gaffey MJ, Weiss LM, Wilczynski SP, Schuuring E, et al. Chromosome 11Q13 Amplification in Head and Neck Squamous Cell Carcinoma. Arch Otolaryngol Neck Surg [Internet]. 1994 Nov 1 [cited 2020 Jun 19];119(1):1238-43. Available from: http://archotol.jamanetwork.com/article.aspx?articleid=621936

61. Brennan JA, Boyle JO, Koch WM, Goodman SN, Hruban RH, Eby YJ, et al. Association Between Cigarette Smoking and Mutation of the p53 Gene in Squamous-Cell Carcinoma of the Head and Neck. 1995;712-7. 
62. Hanel W, Moll UM. Links between mutant p53 and genomic instability. J Cell Biochem. $2012 ; 113(2): 433-9$.

63. Moktar A, Ravoori S, Vadhanam M V., Gairola CG, Gupta RC. Cigarette smoke-induced DNA damage and repair detected by the comet assay in HPV-transformed cervical cells. Int J Oncol. 2009;35(6):1297-304.

64. Menon V, Povirk L. Involvement of p53 in the Repair of DNA Double Strand Breaks: Multifaceted Roles of p53 in Homologous Recombination Repair (HRR) and Non-Homologous End Joining (NHEJ). In: Mutant p53 and MDM2 in Cancer [Internet]. 2014. p. 321-36. Available from: http://link.springer.com/10.1007/978-94-017-9211-0

65. Jin Y, Höglund M, Jin C, Martins C, Wennerberg J, Åkervall J, et al. Fish characterization of head and neck carcinomas reveals that amplification of band $11 \mathrm{q} 13$ is associated with deletion of distal 11q. Genes Chromosom Cancer [Internet]. 1998 Aug 1 [cited 2020 Jun 19];22(4):312-20. Available from: http://doi.wiley.com/10.1002/\%28SICI\%291098-2264\%28199808\%2922\%3A4\%3C312\%3A\%3AAIDGCC7\%3E3.0.CO\%3B2-Y

66. Huang X, Gollin SM, Raja S, Godfrey TE. High-resolution mapping of the 11q13 amplicon and identification of a gene, TAOS1, that is amplified and overexpressed in oral cancer cells. Proc Natl Acad Sci U S A [Internet]. 2002 Aug 20;99(17):11369-74. Available from:

http://www.pubmedcentral.nih.gov/articlerender.fcgi?artid=123263\&tool=pmcentrez\&rendertype=abstrac $\mathrm{t}$

67. Wilkerson PM, Reis-Filho JS. The 11q13-q14 amplicon: clinicopathological correlations and potential drivers. Genes Chromosomes Cancer [Internet]. 2013 Apr [cited 2014 Jun 3];52(4):333-55. Available from: http://onlinelibrary.wiley.com/doi/10.1002/gcc.22037/full

68. Kao-Shan C-S, Fine RL, Whang-Peng J, Lee EC, Chabner BA, Lee EC. Increased Fragile Sites And Sister Chromatid Exchanges In Bone Marrow And Peripheral Blood Of Young Cigarette Smokers. Cancer Res [Internet]. 1987;47(23):6278-82. Available from: http://cancerres.aacrjournals.org/content/47/23/6278.abstract 
69. Rasamny JJ, Allak A, Krook KA, Jo VY, Policarpio-Nicolas ML, Sumner HM, et al. Cyclin D1 and FADD as biomarkers in head and neck squamous cell carcinoma. Otolaryngol Head Neck Surg [Internet]. 2012 Jun;146(6):923-31. Available from: http://www.ncbi.nlm.nih.gov/pubmed/22323434

70. Li M, Cui X, Shen Y, Dong H, Liang W, Chen Y, et al. ORAOV1 overexpression in esophageal squamous cell carcinoma and esophageal dysplasia: A possible biomarker of progression and poor prognosis in esophageal carcinoma. Hum Pathol [Internet]. 2015;46(5):707-15. Available from: http://dx.doi.org/10.1016/j.humpath.2015.01.009

71. Pehkonen H, Lento M, Von Nandelstadh P, Filippou A, Grénman R, Lehti K, et al. Liprin- 1 1 modulates cancer cell signaling by transmembrane protein CD82 in adhesive membrane domains linked to cytoskeleton. Cell Commun Signal. 2018;16(1):1-14.

72. Godse NR, Khan N, Yochum ZA, Gomez-Casal R, Kemp C, Shiwarski DJ, et al. TMEM16A/ANO1 inhibits apoptosis via downregulation of Bim expression. Clin Cancer Res. 2017;23(23):7324-32.

73. Gao L, Lang L, Zhao X, Shay C, Shull AY, Teng Y. FGF19 amplification reveals an oncogenic dependency upon autocrine FGF19/FGFR4 signaling in head and neck squamous cell carcinoma. Oncogene [Internet]. 2019 [cited 2020 Jun 19];38(13):2394-404. Available from: https://pubmed.ncbi.nlm.nih.gov/30518874/

74. Rodrigo JP, Garcia LA, Lazo PS, Ramos S, Suarez C. EMS1 gene amplification correlates with poor prognosis in HNSCC. Otolaryngol Neck Surg. 2006;121(2_suppl):P86-7.

75. Miyamoto R, Uzawa N, Nagaoka S, Nakakuki K, Hirata Y, Amagasa T. Potential marker of oral squamous cell carcinoma aggressiveness detected by fluorescence in situ hybridization in fine-needle aspiration biopsies. Cancer. 2002;95(10):2152-9.

76. van Kempen PMW, Noorlag R, Braunius WW, Moelans CB, Rifi W, Savola S, et al. Clinical relevance of copy number profiling in oral and oropharyngeal squamous cell carcinoma. Cancer Med. 2015;4(10):1525-35.

77. Timpson P, Wilson AS, Lehrbach GM, Sutherland RL, Musgrove EA, Daly RJ. Aberrant expression of cortactin in head and neck squamous cell carcinoma cells is associated with enhanced cell proliferation 
and resistance to the epidermal growth factor receptor inhibitor gefitinib. Cancer Res.

2007;67(19):9304-14.

78. Zhong L-P, Zhu D-W, William WN, Liu Y, Ma J, Yang C-Z, et al. Elevated Cyclin D1 Expression Is Predictive for a Benefit from TPF Induction Chemotherapy in Oral Squamous Cell Carcinoma Patients with Advanced Nodal Disease. Mol Cancer Ther. 2013;12(6):1112-21.

79. Zhang P, Zhang Z, Zhou X, Qiu W, Chen F, Chen W. Identification of genes associated with cisplatin resistance in human oral squamous cell carcinoma cell line. BMC Cancer. 2006;6:1-11. 
Figure Legends

A

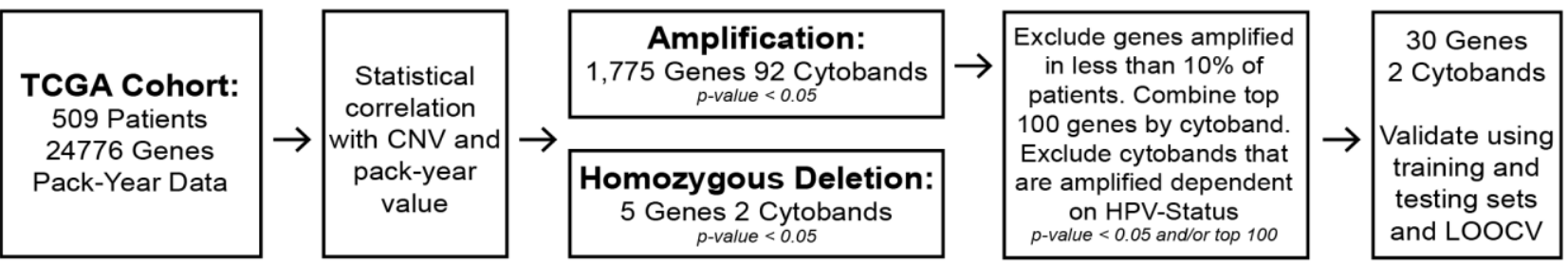

B Amplified Cytobands by HPV-Status

C

Amplified Genes that Negatively Correlate with Pack-Year Value

\begin{tabular}{|l|l|lll|} 
HPV-Positive & \multicolumn{3}{c}{ HPV-Negative } \\
\hline None & $11 q 13.3$ & $3 q 23$ & $3 q 25.33$ & $3 q 27.1$ \\
& $11 q 13.4$ & $3 q 24$ & $3 q 26.1$ & $3 q 26.2$ \\
& $3 q 25.1$ & $3 q 26.2$ & $3 q 26.3$ \\
& $3 q 25.2$ & $3 q 26.31$ & $3 q 28$ \\
& $3 q 25.31$ & $3 q 26.32$ & $3 q 29$ \\
\hline & $3 q 25.32$ & $3 q 26.33$ \\
\hline
\end{tabular}
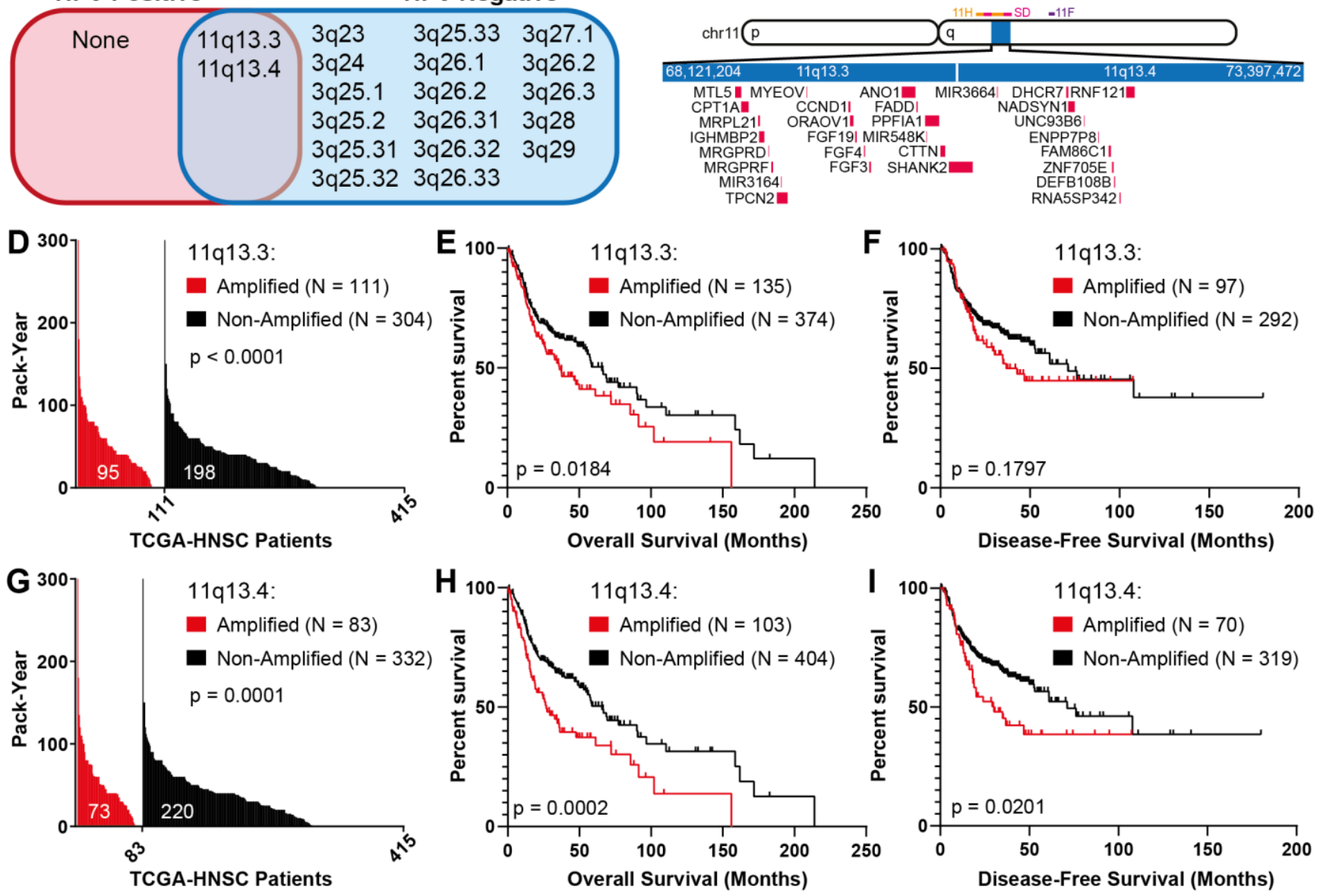

Figure 1. 11q13.3 and 11q13.4 amplification in HNSCC is correlated with genomic instability resultant

from smoking. A. Schematic depicting experimental determination of smoking correlated CNAs from the TCGA-

HNSC dataset containing 509 patients with CNA and pack-year data. B. Segregation of smoking correlated CNAs based on HPV-status. CNAs independent of HPV are denoted in the center of the diagram. C. Diagrammatic representation of chromosome 11 with location of significantly smoking-associated amplified 11q13.3-q13.4 genes. Fragile sites FRA(11H), FRA(11F), and SDs are shown at their respective locations. Loci position and size are drawn to scale based on coordinates from the UCSC Genome Browser. D. Waterfall plot showing patient pack-year distribution stratified by 11 q13.3 cytoband amplification status. E-F. Overall (E) and disease-free (F) survival analysis of patients with 11 q13.3 amplification. G. Waterfall plot showing patient pack- 
year distribution stratified by 11q13.4 amplification status. H-I. Overall (H) and disease-free (I) survival analysis of patients with amplification of 11q13.4.

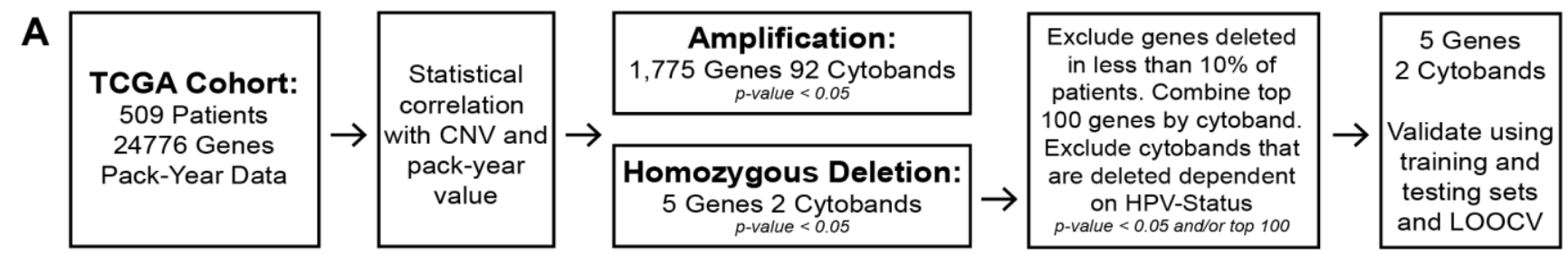

B Deleted Cytobands by HPV-Status
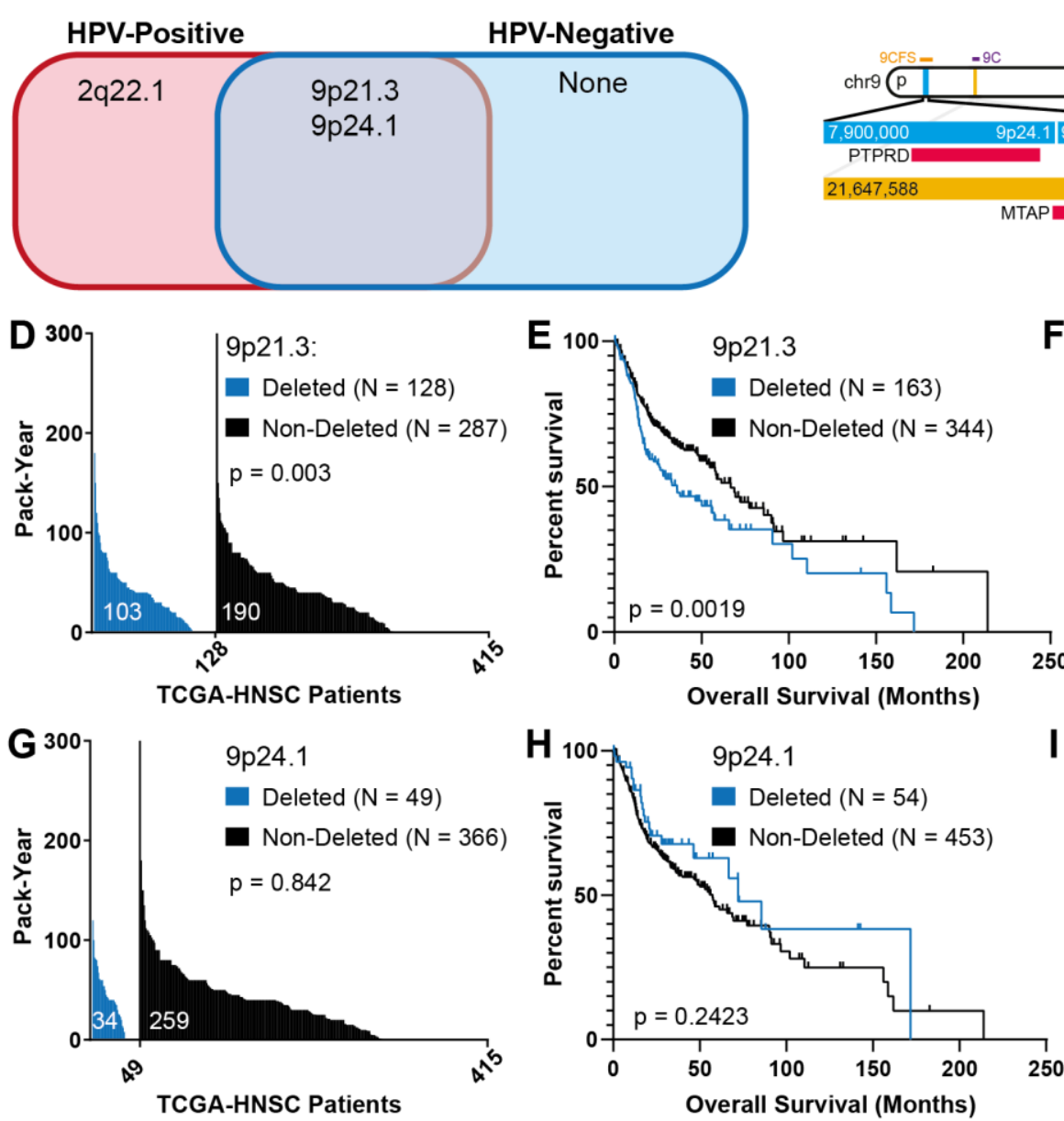

F 100 - $9 \mathrm{p} 21.3$
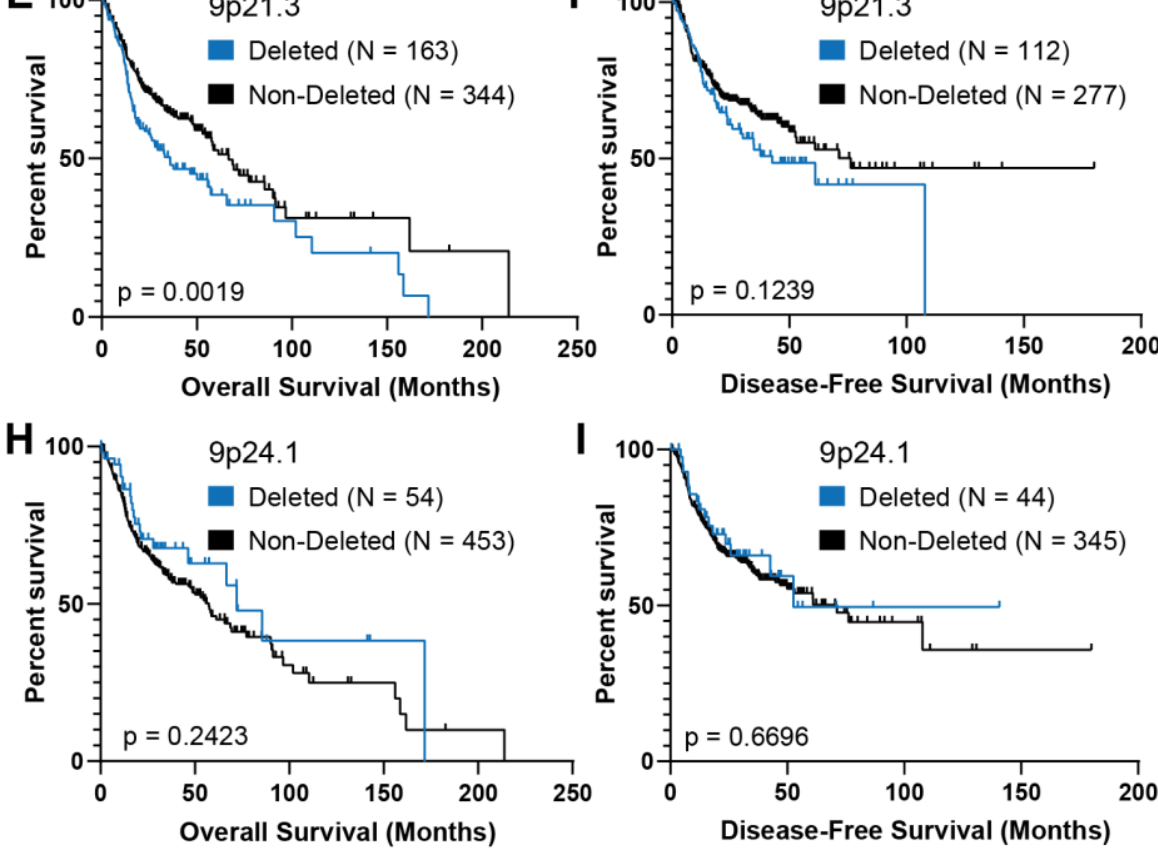

Figure 2. 9p21.3 and 9p24.1 deletion in HNSCC is correlated with patient smoking status. A. Schematic depicting experimental determination of smoking correlated deletions from the TCGA-HNSC dataset. B. Smoking correlated homozygous deletions grouped by HPV-status. CNAs independent of HPV are in the center of the diagram. C. Diagrammatic representation of chromosome 9 with location of significantly smoking-associated deleted 9p21.3 and 9p24.1 genes. Fragile site FRA(9C) and the unnamed CRS (9CRS) are shown. Loci position and size are drawn to scale based on coordinates from UCSC Genome Browser. D. Waterfall plot showing patient pack-year distribution stratified by 9p21.3 cytoband deletion status. E-F. Overall (E) and disease-free (F) survival analysis of patients with 9p21.3 amplification. G. Waterfall plot showing patient pack-year distribution 
stratified by 9p24.1 deletion status. H-I. Overall $(\mathbf{H})$ and disease-free (I) Kaplan-Meier survival analysis of patients with amplification of 9p24.1.
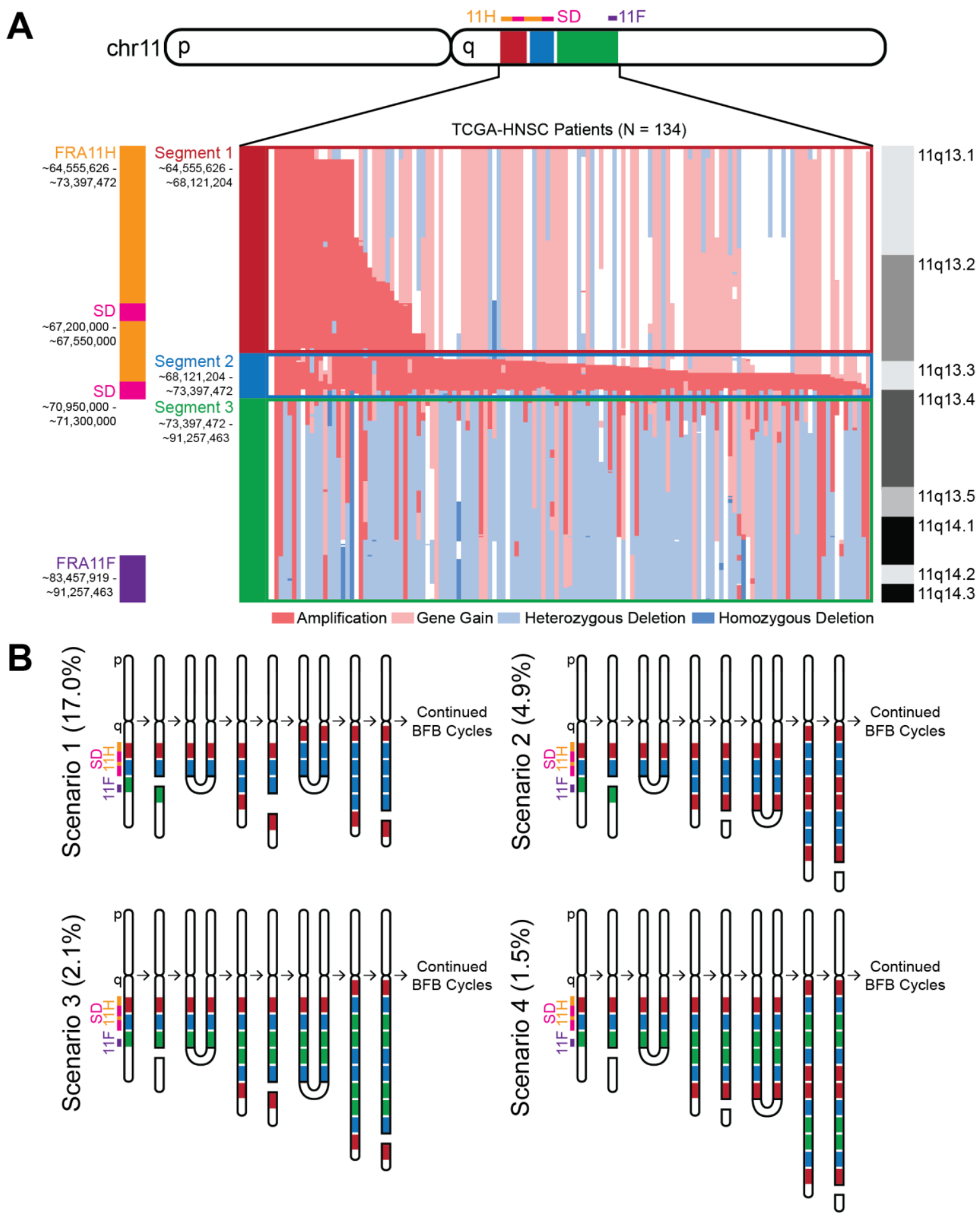

Figure 3. 11q13 Amplification Patterns Identify a Distinct Chromosomal Segment Containing Smoking Overexpression Signature Genes in HNSCC. A. Heat map displaying all 11q13 amplified HNSCC patients aligned by amplicon frequency and size. Patient genomic status was categorized by amplification ( $>2$ extra 
copies; dark red), gene gain (2 extra copies; light red), heterozygous deletion (light blue), and homozygous deletion (dark blue). Fragile sites 11H (FRA11H; orange) and 11F (FRA11F; purple) and segmental duplications (SD; magenta) with respective genomic location and approximate coordinates are displayed at the top and on the left. Corresponding chromosome 11q cytoband positions are located on the right. Patients are grouped by decreasing amplicon length, with conserved breakage coordinates indicated by segment position. Segment 1 (red); segment 2 (blue); segment 3 (green) are noted with their respective estimated chromosomal ranges. B. Frequency of 11q13 amplification scenarios in HNSCC. BFB cycles illustrate each possible amplification event based on chromosomal alignment and frequency of occurrence in the TCGA-HNSC cohort. Fragile site, SD, and amplicon segment locations are color-coded as in $(\mathbf{A})$.

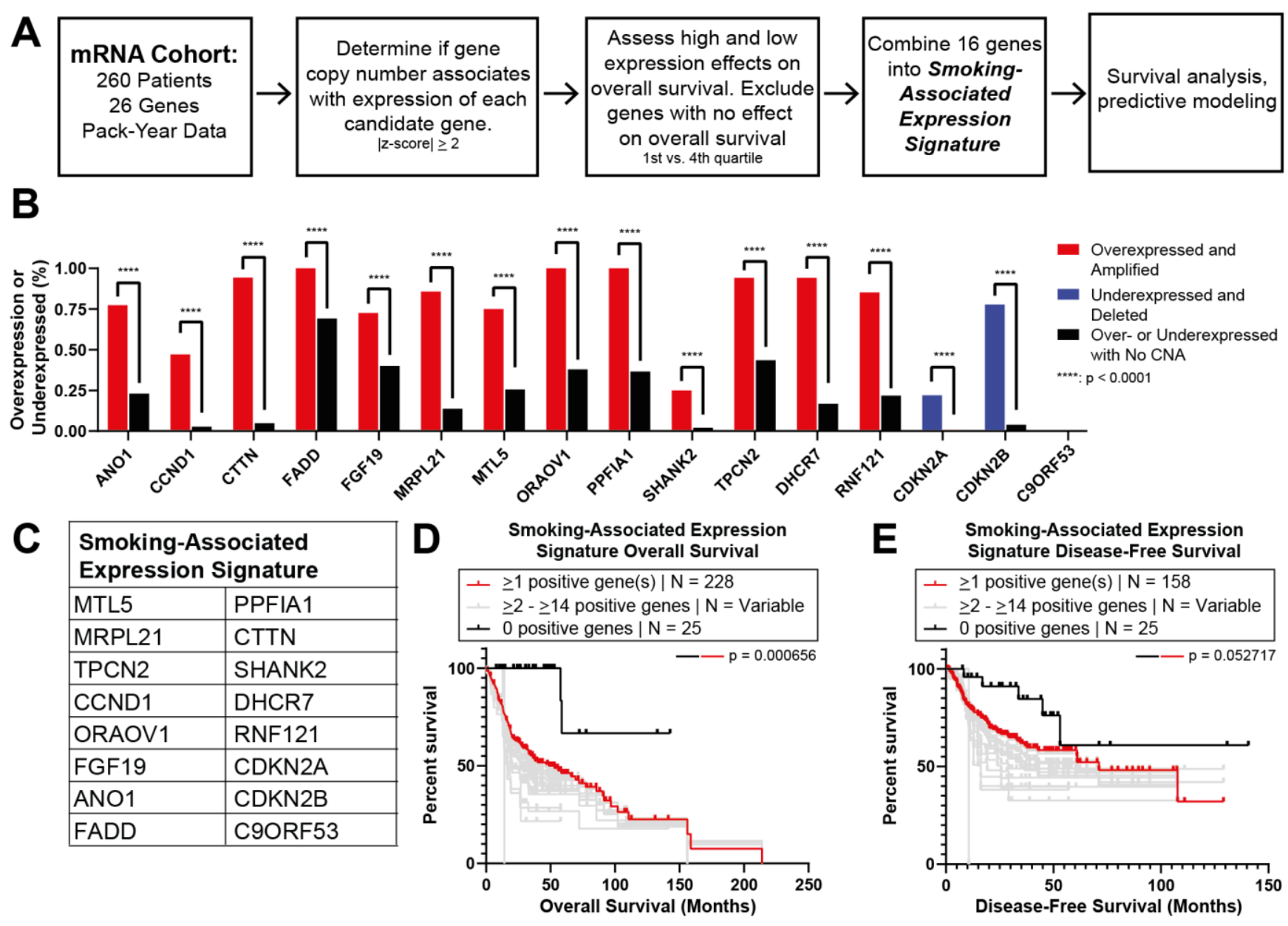

Figure 4. Smoking-associated gene expression identifies a transcriptional signature that negatively impacts HNSCC outcome. A. Schematic diagram showing experimental determination of the SAES from the TCGA-HNSC dataset. 260 patients with full mRNA sequencing and pack-year data were evaluated as indicated. B. Percentage of patients overexpressed and amplified (red), underexpressed and deleted (blue) compared to 
patients that are over- or underexpressed that lack a CNA (black) for each SAES gene. C. Genes with overall survival differences between the first and fourth quartile of transcript expression. The list constitutes the SAES in HNSCC. D-E. Overall (D) and disease-free (E) survival analysis of all patients harboring genes from the SAES. Black lines in each graph represent patients with no positive SAES genes, red line represents patients with one positive SAES gene. Light gray lines represent the additive range of patients with multiple positive SAES genes.
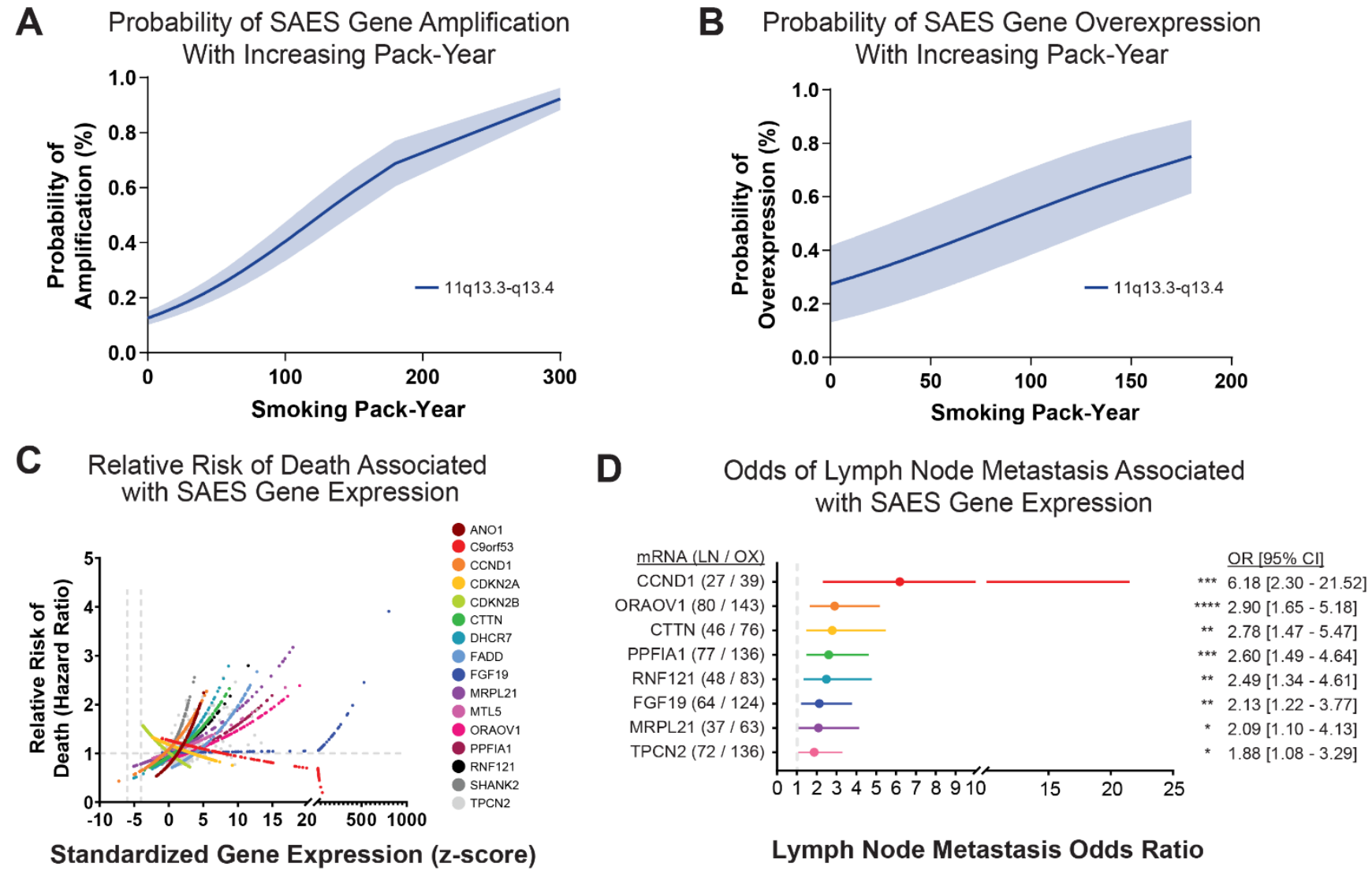

Lymph Node Metastasis Odds Ratio

Figure 5. Predictive modeling and odds analysis of smoking-associated 11q13 genes in HNSCC survival and Iymph node metastasis. A-B. Probability analysis of 11q13.3-q13.4 gene (MTL5, MRPL21, TPCN2, CCND1, ORAOV1, FGF19, ANO1, FADD, PPFIA1, CTTN, SHANK2, DHCR7, and RNF121) amplification (A) and 11q13.3-q13.4 SAES gene (CCND1, CTTN, FADD, FGF19, MRPL21, MTL5, ORAOV1, PPFIA1, SHANK2, and $\mathrm{DHCR}$ ) overexpression (B) in patients with increasing pack-year use. 95\% confidence interval $(95 \% \mathrm{Cl})$ shown in shaded regions. C. Relative risk of death for overexpressed 11q13.3-q13.4 SAES genes (positive slope) and underexpression of 9p21.3 and 9p24.1 SAES genes (negative slope). Left vertical dashed line represents a z-score of -2 (underexpressed). Right vertical dashed line represents a z-score of 2 (overexpressed). Horizontal dashed line represents a relative risk of 1. D. Odds ratio analysis of SAES genes significant for lymph node metastasis due to 11q13.3-q13.4 overexpression. Total patients with lymph node 
metastasis (LN) and total patients with overexpression (OX) are shown for each gene with the corresponding odds ratio (OR) and 95\% $\mathrm{Cl}$. 


\begin{tabular}{|c|c|c|c|c|c|c|c|c|c|}
\hline $\begin{array}{l}\text { Chr11 Amplification } \\
\text { Genomic Alterations }\end{array}$ & $\begin{array}{l}\text { Total } \\
n=509(100 \%)\end{array}$ & $\begin{array}{l}\text { Smokers } \\
n=387(100 \%)\end{array}$ & $\begin{array}{l}\text { Non-Smokers } \\
n=122(100 \%)\end{array}$ & $\begin{array}{l}\text { Total Amplified } \\
n=136(100 \%)\end{array}$ & $\begin{array}{l}\text { Amplified } \\
\text { Smokers } \\
n=120(100 \%)\end{array}$ & $\begin{array}{l}\text { Amplified Non- } \\
\text { Smokers } \\
n=16(100 \%)\end{array}$ & $\begin{array}{l}\text { Total Non- } \\
\text { Amplified } \\
n=373(100 \%)\end{array}$ & $\begin{array}{l}\text { Non-Amplified } \\
\text { Smokers } \\
n=267(100 \%)\end{array}$ & $\begin{array}{l}\text { Non-Amplified } \\
\text { Non-Smokers } \\
n=106(100 \%)\end{array}$ \\
\hline & Mean \pm SD & Mean \pm SD & Mean \pm SD & Mean \pm SD & Mean \pm SD & Mean \pm SD & Mean \pm SD & Mean \pm SD & Mean \pm SD \\
\hline Total SNA & $149.9 \pm 190.2$ & $154.9 \pm 141.8$ & $134.4 \pm 294.8$ & $144.6 \pm 99.58$ & $150.4 \pm 102.9$ & $101.8 \pm 55.87$ & $151.9 \pm 214.2$ & $156.9 \pm 156.5$ & $139.5 \pm 316$ \\
\hline Total CNA (Amp.) & $333.8 \pm 436.6$ & $359.7 \pm 459.3$ & $251.5 \pm 344.4$ & $528.7 \pm 475.3$ * & $541.3 \pm 495.5 \ddagger$ & $434.1 \pm 272.1$ & $262.7 \pm 399.3$ * & $278.1 \pm 417.9 \ddagger$ & $224 \pm 346.8$ \\
\hline Total CNA (Del.) & $97.27 \pm 242$ & $89.74 \pm 202.6$ & $121.1 \pm 338$ & $104.3 \pm 218.2$ & $97.22 \pm 199.4$ & $157 \pm 331.1$ & $94.72 \pm 250.4$ & $86.39 \pm 204.3$ & $115.7 \pm 340.3$ \\
\hline SNA & $\mathbf{n}(\%)$ & n (\%) & n (\%) & $\mathbf{n}(\%)$ & n (\%) & n (\%) & $\mathrm{n}(\%)$ & n (\%) & n (\%) \\
\hline CASP8 & $52(10.2)$ & $32(8.3)^{*}$ & $20(16.4)^{*}$ & $3(2.2)$ * & $2(1.7) \ddagger$ & $1(6.3)$ & $49(13.1)^{*}$ & $30(11.2) \ddagger$ & $19(17.9)$ \\
\hline CDKN2A & $110(21.6)$ & $78(20.2)$ & 32 (26.2) & $29(21.3)$ & 23 (19.2) & $6(37.5)$ & $81(21.7)$ & 55 (20.6) & 26 (24.5) \\
\hline FAT1 & $112(22)$ & $89(23)$ & $23(18.9)$ & $22(16.2)$ & $21(17.5)$ & $1(6.3)$ & $90(24.1)$ & $68(25.5)$ & $22(20.8)$ \\
\hline KMT2D & $57(11.2)$ & $41(10.6)$ & $16(13.1)$ & $1(0.7)$ * & $0(0) \dagger \ddagger$ & $1(6.3) \dagger$ & $56(15)^{*}$ & $41(15.4) \ddagger$ & $15(14.2)$ \\
\hline LRP1B & 86 (16.9) & $76(19.6)$ * & $10(8.2)$ * & $25(18.4)$ & $25(20.8) \dagger$ & $0(0) \dagger$ & $61(16.4)$ & $51(19.1) \dagger$ & $10(9.4) \dagger$ \\
\hline NOTCH1 & $87(17.1)$ & $70(18.1)$ & 17 (13.9) & $22(16.2)$ * & $19(15.8)$ & $3(18.8)$ & $65(17.4)$ * & $51(19.1)$ & $14(13.2)$ \\
\hline NSD1 & 60 (11.8) & $55(14.2)$ * & $5(4.1) *$ & $9(6.6)^{*}$ & $9(7.5) \ddagger$ & $0(0)$ & $51(13.7)$ * & $46(17.2) \dagger \ddagger$ & $5(4.7) \dagger$ \\
\hline PCLO & 79 (15.5) & $66(17.1)$ & $13(10.7)$ & $26(19.1)$ * & 23 (19.2) & $3(18.8)$ & $53(14.2)$ * & $43(16.1)$ & $10(9.4)$ \\
\hline PIK3CA & $88(17.3)$ & $71(18.3)$ & 17 (13.9) & $20(14.7)$ & $17(14.2)$ & $3(18.8)$ & $68(18.2)$ & $54(20.2)$ & 14 (13.2) \\
\hline TP53 & $353(69.4)$ & $275(71.1)$ & $78(63.9)$ & $114(83.8)^{*}$ & $102(85) \ddagger$ & $12(75)$ & $239(64.1)$ * & $173(64.8) \ddagger$ & $66(62.3)$ \\
\hline CNA & n (\%) & n (\%) & n (\%) & n (\%) & n (\%) & n (\%) & n (\%) & $\mathbf{n}(\%)$ & $\mathrm{n}(\%)$ \\
\hline 11q13.3 (Amp.) & $135(26.5)$ & $119(30.7)^{*}$ & $16(13.1)^{*}$ & $135(99.3)^{*}$ & $119(99.2) \ddagger$ & $16(100) \ddagger$ & $0(0)^{*}$ & $0(0) \ddagger$ & $0(0) \ddagger$ \\
\hline $11 \mathrm{q} 13.4$ (Amp.) & $103(20.2)$ & $93(24)$ * & $10(8.2)^{*}$ & $103(75.7)$ * & $93(77.5) \ddagger$ & $10(62.5) \ddagger$ & $0(0)$ * & $0(0) \ddagger$ & $0(0) \ddagger$ \\
\hline $11 \mathrm{q} 13.3$ and $11 \mathrm{q} 13.4(\mathrm{Amp}$ & $102(20)$ & $92(23.8)$ * & $10(8.2) *$ & $102(75)^{*}$ & $92(76.7) \ddagger$ & $10(62.5) \ddagger$ & $0(0)^{*}$ & $0(0) \ddagger$ & $0(0)$ \\
\hline 9q21.3 (Del.) & $164(32.2)$ & $139(35.9)^{*}$ & $25(20.5)$ * & $65(47.8)$ * & $60(50) \ddagger$ & $5(31.3)$ & $99(26.5)$ * & $79(29.6) \dagger \ddagger$ & $20(18.9) \dagger \ddagger$ \\
\hline 9p24.1 (Del.) & $55(10.8)$ & $40(10.3)$ & $15(12.3)$ & $16(11.8)$ & $15(12.5)$ & $1(6.3)$ & 39 (10.5) & $25(9.4)$ & $14(13.2)$ \\
\hline 9q21.3 and 9q24.1 (Del.) & $28(5.5)$ & $22(5.7)$ & $6(4.9)$ & $10(7.4)$ & $10(8.3)$ & $0(0)$ & $18(4.8)$ & $12(4.5)$ & $6(5.7)$ \\
\hline \multirow[t]{2}{*}{$\begin{array}{l}\text { Chr9 Deletion Genomic } \\
\text { Alterations }\end{array}$} & $\begin{array}{l}\text { Total } \\
n=509(100 \%)\end{array}$ & $\begin{array}{l}\text { Smokers } \\
n=387(100 \%)\end{array}$ & $\begin{array}{l}\text { Non-Smokers } \\
n=122(100 \%)\end{array}$ & $\begin{array}{l}\text { Total Deleted } \\
n=191(100 \%)\end{array}$ & $\begin{array}{l}\text { Deleted Smokers } \\
\mathrm{n}=157(100 \%)\end{array}$ & $\begin{array}{l}\text { Deleted Non- } \\
\text { Smokers } \\
n=34(100 \%)\end{array}$ & $\begin{array}{l}\text { Total Non- } \\
\text { Deleted } \\
\mathrm{n}=318(100 \%)\end{array}$ & $\begin{array}{l}\text { Non-Deleted } \\
\text { Smokers } \\
n=230(100 \%)\end{array}$ & $\begin{array}{l}\text { Non-Deleted } \\
\text { Non-Smokers } \\
n=88(100 \%)\end{array}$ \\
\hline & Mean \pm SD & Mean \pm SD & Mean \pm SD & Mean \pm SD & Mean \pm SD & Mean \pm SD & Mean \pm SD & Mean \pm SD & Mean \pm SD \\
\hline Total SNA & $149.9 \pm 190.2$ & $154.9 \pm 141.8$ & $134.4 \pm 294.8$ & $149.8 \pm 137.4$ & $155 \pm 146.3$ & $125.3 \pm 81.85$ & $150 \pm 216.2$ & $154.7 \pm 138.9$ & $137.9 \pm 343.6$ \\
\hline Total CNA (Amp.) & $333.8 \pm 436.6$ & $359.7 \pm 459.3$ & $251.5 \pm 344.4$ & $388.4 \pm 508.3$ & $406.7 \pm 519.4$ & $304.2 \pm 451$ & $300.9 \pm 384.4$ & $327.6 \pm 411.3$ & $231.2 \pm 293.9$ \\
\hline Total CNA (Del.) & $97.27 \pm 242$ & $89.74 \pm 202.6$ & $121.1 \pm 338$ & $120.8 \pm 202.9$ & $110.5 \pm 193.3$ & $168.7 \pm 240$ & $83.1 \pm 262$ & $75.6 \pm 207.9$ & $102.7 \pm 368.6$ \\
\hline SNA & n (\%) & $\mathrm{n}(\%)$ & n (\%) & $\mathbf{n}(\%)$ & n (\%) & n (\%) & n (\%) & $\mathbf{n}(\%)$ & n (\%) \\
\hline CASP8 & $52(10.2)$ & $32(8.3)^{*}$ & $20(16.4)^{*}$ & $22(11.5)$ & $12(7.6) \dagger$ & $10(29.4) \dagger \ddagger$ & $30(9.4)$ & $20(8.7)$ & $10(11.4) \ddagger$ \\
\hline CDKN2A & $110(21.6)$ & $78(20.2)$ & $32(26.2)$ & $17(8.9)$ * & $12(7.6) \ddagger$ & $5(14.7)$ & $93(29.2)$ * & $66(28.7) \ddagger$ & $27(30.7)$ \\
\hline FAT1 & $112(22)$ & $89(23)$ & $23(18.9)$ & $46(24.1)$ & $32(20.4) \dagger$ & $14(41.2) \dagger \ddagger$ & $66(20.8)$ & $57(24.8) \dagger$ & $9(10.2) \dagger \ddagger$ \\
\hline KMT2D & $81(15.9)$ & $65(16.8)$ & $16(13.1)$ & $33(17.3)$ & $29(18.5)$ & $4(11.8)$ & $48(15.1)$ & $36(15.7)$ & $12(13.6)$ \\
\hline LRP1B & 86 (16.9) & $76(19.6)$ * & $10(8.2)$ * & 31 (16.2) & $28(17.8)$ & $3(8.8)$ & $55(17.3)$ & $48(20.9) \dagger$ & $7(8) \dagger$ \\
\hline NOTCH1 & $87(17.1)$ & $70(18.1)$ & $17(13.9)$ & $44(23)^{*}$ & $37(23.6) \ddagger$ & $7(20.6)$ & $43(13.5)$ * & $33(14.3) \ddagger$ & $10(11.4)$ \\
\hline NSD1 & $60(11.8)$ & $55(14.2)$ * & $5(4.1)^{*}$ & $22(11.5)$ & $21(13.4)$ & $1(2.9)$ & 38 (11.9) & $34(14.8) \dagger$ & $4(4.5) \dagger$ \\
\hline PCLO & 79 (15.5) & $66(17.1)$ & $13(10.7)$ & $28(14.7)$ & $25(15.9)$ & $3(8.8)$ & $51(16)$ & $41(17.8)$ & $10(11.4)$ \\
\hline PIK3CA & $88(17.3)$ & $71(18.3)$ & $17(13.9)$ & $25(13.1)$ & $19(12.1) \ddagger$ & $6(17.6)$ & $63(19.8)$ & $52(22.6) \dagger$ & $11(12.5) \dagger$ \\
\hline TP53 & $353(69.4)$ & $275(71.1)$ & $78(63.9)$ & $146(76.4)^{*}$ & $127(80.9) \dagger \ddagger$ & $19(55.9) \dagger$ & $207(65.1)$ * & $148(64.3) \ddagger$ & $59(67)$ \\
\hline CNA & n (\%) & n (\%) & n (\%) & n (\%) & n (\%) & n (\%) & n (\%) & n (\%) & $\mathrm{n}(\%)$ \\
\hline 11q13.3 (Amp.) & $135(26.5)$ & $119(30.7)^{*}$ & $16(13.1)^{*}$ & $70(36.6)^{*}$ & $64(40.8) \dagger \ddagger$ & $6(17.6) \dagger$ & $65(20.4)^{*}$ & $55(23.9) \dagger \ddagger$ & $10(11.4)$ \\
\hline 11q13.4 (Amp.) & $103(20.2)$ & $93(24) *$ & $10(8.2)^{*}$ & $52(27.2)$ * & $48(30.6) \dagger \ddagger$ & $4(11.8) \dagger$ & $51(16)^{*}$ & $45(19.6) \dagger \ddagger$ & $6(6.8)$ \\
\hline $11 \mathrm{q} 13.3$ and $11 \mathrm{q} 13.4(\mathrm{Amp}$ & $102(20)$ & $92(23.8)$ * & $10(8.2) *$ & $51(26.7)^{*}$ & $47(29.9) \dagger \ddagger$ & $4(11.8) \dagger$ & $51(16)$ * & $45(19.6) \dagger \ddagger$ & $6(6.8)$ \\
\hline 9q21.3 (Del.) & $164(32.2)$ & $139(35.9)$ * & $25(20.5)$ * & $164(85.9)$ * & $139(88.5) \dagger$ & $25(73.5) \dagger \ddagger$ & $0(0) *$ & $0(0) \ddagger$ & $0(0) \ddagger$ \\
\hline 9p24.1 (Del.) & $55(10.8)$ & $40(10.3)$ & 15 (12.3) & $55(28.8)$ * & $40(25.5) \ddagger$ & $15(44.1) \dagger \ddagger$ & $0(0)$ * & $0(0) \ddagger$ & $0(0) \ddagger$ \\
\hline 9q21.3 and 9q24.1 (Del.) & $28(5.5)$ & $22(5.7)$ & $6(4.9)$ & $28(14.7)$ * & $22(14) \ddagger$ & $6(17.6) \ddagger$ & $0(0)$ * & $0(0) \ddagger$ & $0(0) \ddagger$ \\
\hline
\end{tabular}

${ }^{*} \mathrm{p}<0.05$ Total Amplified/Deleted vs Total Non-Amplified/Deleted or Total Smoker vs Total Non-Smoker by Pearson chi-squared test (bottom)

$\dagger p<0.05$ Amplified/Deleted Smoker vs Amplified/Deleted Non-Smoker or Non-Amplified/Deleted Smoker vs Non-Amplified/Deleted Non-Smoker by one-way ANOVA (top) or Pearson chi-squared test (bottom) $\ddagger p<0.05$ Amplified/Deleted Smoker vs Non-Amplified/Deleted Smoker or Amplified/Deleted Non-Smoker vs Non-Amplified/Deleted Non-Smoker by one-way ANOVA (top) or Pearson chi-squared test (bottom) SD: Standard Deviation

Smokers: Pack-year $>0$ or Patient Smoking History; Non-Smokers: Pack-year $=0$ or no reported Patient Smoking History 
Table 1. Mutational Frequency of Common HNSCC-associated Genes in Smoking and non-Smoking

Patients with 11q13.3 and 11q13.4 Gene Amplification 


\section{Study 2 Supplementary Materials: Copy Number Alterations Identify a Smoking-Associated Expression Signature Predictive of Poor Outcome in Head and Neck Squamous Cell Carcinoma}

Brenen W. Papenberg ${ }^{1}$, James Ingles ${ }^{2}$, Si Gao², Jun Feng ${ }^{3}$, Jessica L. Allen ${ }^{1}$, Steven M. Markwell ${ }^{1}$, Erik T. Interval ${ }^{4}$, Phillip A. Montague ${ }^{4}$, Sijin Wen ${ }^{2}$ and Scott A. Weed ${ }^{1^{*}}$

${ }^{1}$ Department of Biochemistry, Program in Cancer Cell Biology, ${ }^{2}$ Department of Biostatistics, ${ }^{3}$ Department of Statistics, ${ }^{4}$ Department of Otolaryngology, Head and Neck Surgery, West Virginia University, Morgantown, West Virginia, 26506

\begin{tabular}{|c|c|c|c|c|c|c|c|c|c|}
\hline $\begin{array}{l}\text { Chr11 Amplification } \\
\text { Patient Characteristics }\end{array}$ & $\begin{array}{l}\text { Total } \\
n=509(100 \%)\end{array}$ & $\begin{array}{l}\text { Total Smokers } \\
n=364(100 \%)\end{array}$ & $\begin{array}{l}\text { Total Non-Smokers } \\
n=145(100 \%)\end{array}$ & $\begin{array}{l}\text { Total Amplified } \\
n=136(100 \%)\end{array}$ & $\begin{array}{l}\text { Amplified Smokers } \\
n=120(100 \%)\end{array}$ & $\begin{array}{l}\text { Amplified Non- } \\
\text { Smokers } \\
n=16(100 \%)\end{array}$ & $\begin{array}{l}\text { Total Non- } \\
\text { Amplified } \\
n=373(100 \%)\end{array}$ & $\begin{array}{l}\text { Non-Amplified } \\
\text { Smokers } \\
n=244(100 \%)\end{array}$ & $\begin{array}{l}\text { Non-Amplified } \\
\text { Non-Smokers } \\
n=129(100 \%)\end{array}$ \\
\hline & Mean \pm SD & Mean \pm SD & Mean \pm SD & Mean \pm SD & Mean \pm SD & Mean \pm SD & Mean \pm SD & Mean \pm SD & Mean \pm SD \\
\hline Age (years) & $60.7 \pm 12.2$ & $61.2 \pm 11.0$ & $59.1 \pm 15.1$ & $59.8 \pm 12.4$ & $60.1 \pm 12.3$ & $57.9 \pm 13.4$ & $61 \pm 12.2$ & $61.7 \pm 10.4$ & $59.3 \pm 15.4$ \\
\hline \multicolumn{10}{|l|}{ Smoking Usage } \\
\hline Pack-years & $32.4 \pm 36.3$ & $45.9 \pm 35.3^{*}$ & $0 \pm 0$ * & $46.6 \pm 41.7^{*}$ & $54.4 \pm 40.1 \dagger$ & $0 \pm 0 \dagger$ & $27.1 \pm 32.6^{*}$ & $41.7 \pm 32.2 \dagger$ & $0 \pm 0 \dagger$ \\
\hline \multicolumn{10}{|l|}{ Alcohol Usage } \\
\hline Weekly Drinks & $20.3 \pm 29.9$ & $24.4 \pm 31.7^{*}$ & $5.0 \pm 13.8$ * & $26.6 \pm 30.8$ & $28.5 \pm 31.0 \dagger$ & $0 \pm 0 \dagger$ & $17.7 \pm 29.2$ & $22.3 \pm 31.9 \dagger$ & $5.5 \pm 14.4 \dagger$ \\
\hline \multirow[t]{2}{*}{ Survival (months) } & $30.3 \pm 28.5$ & $30.6 \pm 29.8$ & $29.1 \pm 23.9$ & $29.3 \pm 27.3$ & $29.7 \pm 28.1$ & $26.5 \pm 20.5$ & $30.6 \pm 28.9$ & $31.1 \pm 30.5$ & $29.5 \pm 24.5$ \\
\hline & n (\%) & n (\%) & n (\%) & n (\%) & n (\%) & n (\%) & n (\%) & n (\%) & n (\%) \\
\hline \multicolumn{10}{|l|}{ Sex } \\
\hline Male & 375 (73.7) & $290(79.7)^{*}$ & $85(58.6)^{*}$ & $106(77.9)$ & $97(80.8) \dagger$ & $9(56.3) \dagger$ & $269(72.1)$ & $193(79.1) \dagger$ & $76(58.9) \dagger$ \\
\hline Female & $134(26.3)$ & $74(20.3)$ * & $60(41.4)^{*}$ & $30(22.1)$ & $23(19.2) \dagger$ & $7(43.8) \dagger$ & $104(27.9)$ & $51(20.9) \dagger$ & $53(41.1) \dagger$ \\
\hline \multicolumn{10}{|l|}{ AJCC Stage } \\
\hline 1 & $26(5.1)$ & $16(4.4)$ & $10(6.9)$ & $6(4.4)$ & $6(5)$ & $0(0)$ & $20(5.4)$ & $10(4.1)$ & $10(7.8)$ \\
\hline$\|$ & $83(16.3)$ & $57(15.7)$ & $26(17.9)$ & $17(12.5)$ & $15(12.5)$ & $2(12.5)$ & $66(17.7)$ & $42(17.2)$ & $24(18.6)$ \\
\hline III & $95(18.7)$ & 67 (18.4) & $28(19.3)$ & $16(11.8)$ & $15(12.5)$ & $1(6.3)$ & $79(21.2)$ & $52(21.3)$ & 27 (20.9) \\
\hline IV & $305(59.9)$ & $224(61.5)$ & $81(55.9)$ & $97(71.3)^{*}$ & $84(70)$ & $13(81.3)$ & $208(55.8)$ * & $140(57.4)$ & 68 (52.7) \\
\hline \multicolumn{10}{|l|}{ N Status } \\
\hline N Positive & $255(50.1)$ & $189(51.9)$ & 66 (45.5) & $82(60.3)$ * & $73(60.8) \ddagger$ & $9(56.3)$ & $173(46.4)$ * & $116(47.5) \ddagger$ & $57(44.2)$ \\
\hline N Negative & $182(35.8)$ & 127 (34.9) & 55 (37.9) & $37(27.2)$ & $30(25)$ & $7(43.8)$ & 145 (38.9) & $97(39.8)$ & 48 (37.2) \\
\hline Missing & $72(14.1)$ & $48(13.2)$ & 24 (16.6) & 17 (12.5) & $17(14.2)$ & $0(0)$ & $55(14.7)$ & 31 (12.7) & 24 (18.6) \\
\hline \multicolumn{10}{|l|}{ HPV Status } \\
\hline Positive & 98 (19.3) & $59(16.2)^{\star}$ & $39(26.9)^{*}$ & $17(12.5)^{*}$ & $15(12.5)$ & $2(12.5)$ & $81(21.7)^{*}$ & $44(18) \dagger$ & $37(28.7) \dagger$ \\
\hline Negative & $409(80.4)$ & $304(83.5)$ * & $105(72.4)^{*}$ & $119(87.5)$ * & $105(87.5)$ & $14(87.5)$ & $290(77.7)$ * & $199(81.6) \dagger$ & $91(70.5) \dagger$ \\
\hline Missing & $2(0.4)$ & $1(0.3)$ & $1(0.7)$ & $0(0)$ & $0(0)$ & $0(0)$ & $2(0.5)$ & $1(0.4)$ & $1(0.8)$ \\
\hline \multirow[t]{2}{*}{$\begin{array}{l}\text { Chr9 Deletion Patient } \\
\text { Characteristics }\end{array}$} & $\begin{array}{l}\text { Total } \\
n=509(100 \%)\end{array}$ & $\begin{array}{l}\text { Total Smokers } \\
n=360(100 \%)\end{array}$ & $\begin{array}{l}\text { Total Non-Smokers } \\
n=149(100 \%)\end{array}$ & $\begin{array}{l}\text { Total Deleted } \\
n=191(100 \%)\end{array}$ & $\begin{array}{l}\text { Deleted Smokers } \\
n=149(100 \%)\end{array}$ & $\begin{array}{l}\text { Deleted Non- } \\
\text { Smokers } \\
n=42(100 \%) \\
\end{array}$ & $\begin{array}{l}\text { Total Deleted } \\
\mathrm{n}=\mathbf{3 1 8}(100 \%)\end{array}$ & $\begin{array}{l}\text { Non-Deleted } \\
\text { Smokers } \\
n=211(100 \%) \\
\end{array}$ & $\begin{array}{l}\text { Non-Deleted Non- } \\
\text { Smokers } \\
n=107(100 \%) \\
\end{array}$ \\
\hline & Mean \pm SD & Mean \pm SD & Mean \pm SD & Mean \pm SD & Mean \pm SD & Mean \pm SD & Mean \pm SD & Mean \pm SD & Mean \pm SD \\
\hline Age (years) & $60.7 \pm 12.2$ & $61.2 \pm 11.0$ & $59.1 \pm 15.1$ & $61.5 \pm 11.8$ & $60.7 \pm 11.3$ & $65.5 \pm 13.6 \ddagger$ & $60.2 \pm 12.3$ & $61.5 \pm 10.9 \dagger$ & $56.6 \pm 15.0 \dagger \ddagger$ \\
\hline \multicolumn{10}{|l|}{ Smoking Usage } \\
\hline Pack-years & $32.4 \pm 36.3$ & $45.9 \pm 35.3^{*}$ & $0 \pm 0$ * & $34.5 \pm 31.4$ & $44.3 \pm 28.8 \dagger$ & $0 \pm 0 \dagger$ & $31.2 \pm 38.9$ & $47.0 \pm 39.2 \dagger$ & $0 \pm 0 \dagger$ \\
\hline \multicolumn{10}{|l|}{ Alcohol Usage } \\
\hline Weekly Drinks & $20.3 \pm 29.9$ & $24.4 \pm 31.7^{*}$ & $5.0 \pm 13.8^{*}$ & $21.2 \pm 24.0$ & $23.6 \pm 24.3$ & $1.1 \pm 2.45$ & $19.8 \pm 32.7$ & $24.9 \pm 35.9 \dagger$ & $5.8 \pm 15.1 \dagger$ \\
\hline \multirow[t]{2}{*}{ Survival (months) } & $30.3 \pm 28.5$ & $30.6 \pm 29.8$ & $29.2 \pm 23.9$ & $29.4 \pm 29.3$ & $29.5 \pm 29.5$ & $28.9 \pm 28.9$ & $30.8 \pm 28.0$ & $31.4 \pm 30.0$ & $29.1 \pm 21.9$ \\
\hline & n (\%) & n (\%) & n (\%) & n (\%) & n (\%) & n (\%) & n (\%) & n (\%) & n (\%) \\
\hline \multicolumn{10}{|l|}{ Sex } \\
\hline Male & 375 (73.7) & $288(80)$ * & $87(58.4)^{\star}$ & $131(68.6)$ & $114(76.5) \dagger$ & $17(40.5) \dagger \ddagger$ & $244(76.7)$ & $174(82.5) \dagger$ & 70 (65.4) † \\
\hline Female & $134(26.3)$ & $72(20)^{*}$ & $62(41.6)^{*}$ & $60(31.4)$ & $35(23.5) \dagger$ & $25(59.5) \dagger \ddagger$ & $74(23.3)$ & $37(17.5) \dagger$ & $37(34.6) \dagger$ \\
\hline \multicolumn{10}{|l|}{ AJCC Stage } \\
\hline 1 & $26(5.1)$ & $15(4.2)$ & $11(7.4)$ & $9(4.7)$ & $6(4)$ & $3(7.1)$ & $17(5.3)$ & $9(4.3)$ & $8(7.5)$ \\
\hline$\|$ & 83 (16.3) & $56(15.6)$ & $27(18.1)$ & $30(15.7)$ & $23(15.4)$ & $7(16.7)$ & $53(16.7)$ & $33(15.6)$ & $20(18.7)$ \\
\hline III & $95(18.7)$ & $67(18.6)$ & $28(18.8)$ & $31(16.2)$ & $22(14.8)$ & $9(21.4)$ & $64(20.1)$ & $45(21.3)$ & $19(17.8)$ \\
\hline IV & $305(59.9)$ & $222(61.7)$ & $83(55.7)$ & $121(63.4)$ & $98(65.8)$ & $23(54.8)$ & $184(57.9)$ & $124(58.8)$ & $60(56.1)$ \\
\hline \multicolumn{10}{|l|}{ N Status } \\
\hline N Positive & $255(50.1)$ & $188(52.2)$ & $67(45)$ & $97(50.8)$ & $79(53)$ & $18(42.9)$ & $158(49.7)$ & $109(51.7)$ & $49(45.8)$ \\
\hline N Negative & $182(35.8)$ & $124(34.4)$ & $58(38.9)$ & $69(36.1)$ & $54(36.2)$ & $15(35.7)$ & $113(35.5)$ & $70(33.2)$ & $43(40.2)$ \\
\hline Missing & $72(14.1)$ & $48(13.3)$ & $24(16.1)$ & $25(13.1)$ & $16(10.7)$ & $9(21.4)$ & $47(14.8)$ & $32(15.2)$ & $15(14)$ \\
\hline \multicolumn{10}{|l|}{ HPV Status } \\
\hline Positive & $98(19.3)$ & $59(16.4) *$ & $39(26.2)^{*}$ & $12(6.3)^{*}$ & $11(7.4) \ddagger$ & $1(2.4) \dagger \ddagger$ & $86(27)$ & $48(22.7) \dagger \ddagger$ & 38 (35.5) † \\
\hline Negative & $409(80.4)$ & $300(83.3)$ * & $109(73.2)$ * & $179(93.7)$ * & $138(92.6) \ddagger$ & $41(97.6) \dagger \ddagger$ & 230 (72.3) & $162(76.8) \dagger \ddagger$ & $68(63.6) \dagger$ \\
\hline Missing & $2(0.4)$ & $1(0.3)$ & $1(0.7)$ & $0(0)$ & $0(0)$ & $0(0)$ & $2(0.6)$ & $1(0.5)$ & $1(0.9)$ \\
\hline \multicolumn{10}{|c|}{${ }^{*} \mathrm{p}<0.05$ Total Amplified/Deleted vs. Total Non-Amplified/Deleted or Total Smoker vs Total Non-Smoker by Pearson chi-squared test (bottom) } \\
\hline $\begin{array}{l}\text { † } p<0.05 \mathrm{Amplified} / \text { Deletec } \\
\pm \mathrm{p}<0.05 \mathrm{Am} \text { plified/Deletec }\end{array}$ & $\begin{array}{l}\text { Smoker vs Ampli } \\
\text { Smoker vs Non- }\end{array}$ & $\begin{array}{l}\text { lified/Deleted Non-S } \\
\text {-Amplified/Deleted }\end{array}$ & $\begin{array}{l}\text { moker or Non-Amplified } \\
\text { moker or Amplified/Dele }\end{array}$ & $\begin{array}{l}\text { d/Deleted Smoker v } \\
\text { eted Non-Smoker v }\end{array}$ & $\begin{array}{l}\text { Non-Amplified/Deleted } \\
\text { Non-Amplified/Deleted }\end{array}$ & Non-Smoker by on & vay ANOVA (top) 0 & $\begin{array}{l}\text { Pearson chi-squa } \\
\text { Pearson chi-squa }\end{array}$ & test (bottom) \\
\hline SD: Standard Deviation & & & & & & & & & \\
\hline Smokers: Pack-year $>0$ o & r Patient Smoking & g History; Non-Smo & ers: Pack-year $=0$ or $n$ & רo reported Patien & moking History & & & & \\
\hline
\end{tabular}

Supplementary Table S1. Clinical Characteristics of HNSCC Patients by Smoking, 11q13.3 and

11q13.4 Cytoband Amplification Status 
Supplementary Table S2. Gene GISTIC2 Value and Pack-Year Linear Regression Analysis. Related

to Figures 1 and 2. Available on WVU Library EDT Website.

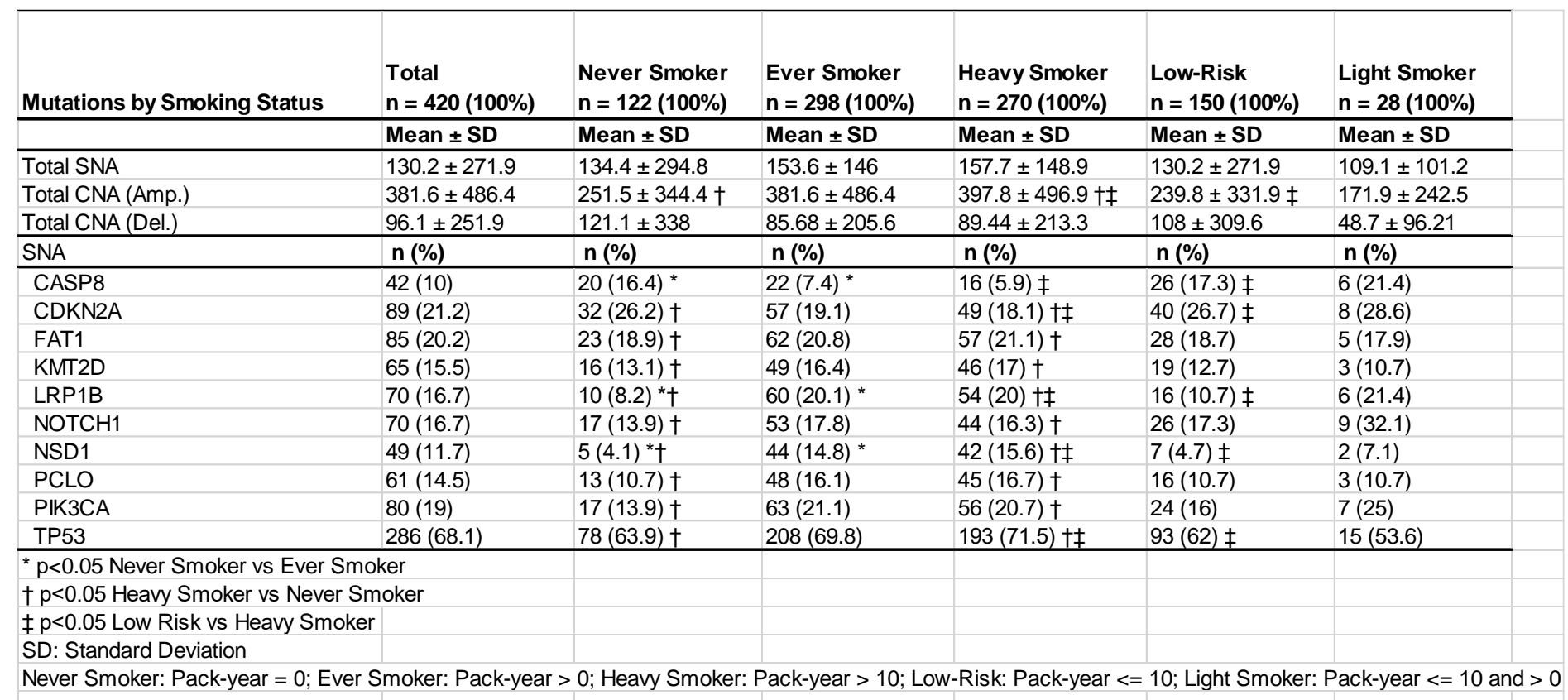

\section{Supplementary Table S3. Mutational Frequency of common HNSCC-associated Genes by}

\section{Smoking Exposure. Related to Table 1.}




\begin{tabular}{|c|c|c|c|c|c|c|c|c|c|}
\hline 11q13.3 Gene Expression & Total & Total Smokers & Total Non-Smokers & Amplified & Amplified Smokers & Amplified Non-Smokers & Non-Amplified & $\begin{array}{l}\text { Non-Amplified } \\
\text { Smokers }\end{array}$ & $\begin{array}{l}\text { Non-Amplified } \\
\text { Non-Smokers }\end{array}$ \\
\hline & OX / Amp (\%) & OX / Amp (\%) & OX / Amp (\%) & OX / Amp (\%) & OX / Amp (\%) & OX / Amp (\%) & OX/Amp (\%) & OX / Amp (\%) & OX / Amp (\%) \\
\hline \multicolumn{10}{|l|}{ Overexpressed } \\
\hline ANO1 & 74 / 196 (37.8) & $57 / 148$ (38.5) & $17 / 48(35.4)$ & $41 / 53(77.4)$ * & $37 / 48(77.1) \ddagger$ & $4 / 5(80) \ddagger$ & $33 / 143(23.1)$ * & $20 / 100(20)$ & $13 / 43(30.2) \ddagger$ \\
\hline CCND1 & 28 / 196 (14.3) & $25 / 148(16.9)$ & $3 / 48(6.3)$ & $24 / 51(47.1)$ * & $22 / 46(47.8) \ddagger$ & $2 / 5(40) \ddagger$ & $4 / 145(2.8)^{*}$ & $3 / 102$ (2.9) & $1 / 43(2.3) \ddagger$ \\
\hline CPT1A & $21 / 196(10.7)$ & $19 / 148(12.8)$ & $2 / 48(4.2)$ & $11 / 24(45.8)$ * & $11 / 22(50) \ddagger$ & $0 / 2(0)$ & $10 / 172(5.8)$ * & $8 / 126(6.3)$ & $2 / 46(4.3)$ \\
\hline CTTN & 57 / 196 (29.1) & 48 / 148 (32.4) & 9 / 48 (18.8) & $50 / 53(94.3)$ * & $45 / 48(93.8) \ddagger$ & $5 / 5(100) \ddagger$ & $7 / 143(4.9)$ * & $3 / 100(3)$ & $4 / 43(9.3) \ddagger$ \\
\hline FADD & 152 / $196(77.6)$ & $117 / 148$ (79.1) & 35 / 48 (72.9) & $53 / 53(100)$ * & $48 / 48(100) \ddagger$ & $5 / 5(100)$ & $99 / 143(69.2)$ * & 69 / 100 (69) & $30 / 43(69.8)$ \\
\hline FGF19 & 95 / 196 (48.5) & $78 / 148(52.7)^{*}$ & $17 / 48(35.4)^{*}$ & $37 / 51(72.5)$ * & $37 / 46(80.4) \dagger$ & $0 / 5(0)$ & $58 / 145(40)^{*}$ & $41 / 102$ (40.2) & $17 / 43(39.5)$ \\
\hline FGF3 & $13 / 196(6.6)$ & $13 / 148(8.8)^{*}$ & $0 / 48(0) *$ & $9 / 51(17.6)^{*}$ & $9 / 46(19.6) \ddagger$ & $0 / 5(0)$ & $4 / 145(2.8)$ * & 4 / $102(3.9)$ & $0 / 43(0)$ \\
\hline FGF4 & 16 / 196 (8.2) & $11 / 148(7.4)$ & 5 / 48 (10.4) & $7 / 51$ (13.7) & $7 / 46(15.2) \ddagger$ & $0 / 5(0)$ & $9 / 145$ (6.2) & 4 / 102 (3.9) & $5 / 43(11.6)$ \\
\hline IGHMBP2 & $62 / 196(31.6)$ & $49 / 148(33.1)$ & $13 / 48(27.1)$ & $26 / 30(86.7)$ * & $24 / 28(85.7) \ddagger$ & $2 / 2(100) \ddagger$ & $36 / 166(21.7)$ * & $25 / 120(20.8)$ & $11 / 46(23.9) \ddagger$ \\
\hline MRGPRD & 38 / 196 (19.4) & $30 / 148(20.3)$ & $8 / 48(16.7)$ & $16 / 34(47.1)$ * & $15 / 32(46.9) \ddagger$ & $1 / 2(50)$ & $22 / 162(13.6)$ * & $15 / 116(12.9)$ & $7 / 46$ (15.2) \\
\hline MRGPRF & $9 / 196(4.6)$ & $7 / 148(4.7)$ & $2 / 48(4.2)$ & $2 / 34(5.9)$ & $2 / 32(6.3)$ & $0 / 2(0)$ & $7 / 162(4.3)$ & $5 / 116(4.3)$ & $2 / 46(4.3)$ \\
\hline MRPL21 & 47 / 196 (24) & $40 / 148(27)$ & 7 / 48 (14.6) & $24 / 28(85.7)$ * & $23 / 26(88.5) \ddagger$ & $1 / 2(50)$ & $23 / 168(13.7)$ * & 17 / 122 (13.9) & $6 / 46(13)$ \\
\hline MTL5 & $60 / 196$ (30.6) & $52 / 148(35.1)$ * & $8 / 48(16.7)^{*}$ & $15 / 20(75)$ * & $14 / 18(77.8) \ddagger$ & $1 / 2(50)$ & $45 / 176(25.6)$ * & $38 / 130(29.2)$ & $7 / 46$ (15.2) \\
\hline MYEOV & $5 / 196(2.6)$ & $5 / 148(3.4)$ & $0 / 48(0)$ & $4 / 46(8.7)^{*}$ & $4 / 43(9.3) \ddagger$ & $0 / 3(0)$ & $1 / 150(0.7)^{*}$ & $1 / 105(1)$ & $0 / 45(0)$ \\
\hline ORAOV1 & $106 / 196(54.1)$ & 84 / 148 (56.8) & 22 / $48(45.8)$ & $51 / 51(100)^{*}$ & $46 / 46(100) \ddagger$ & $5 / 5(100) \ddagger$ & $55 / 145(37.9)$ * & $38 / 102(37.3)$ & $17 / 43(39.5) \ddagger$ \\
\hline PPFIA1 & $106 / 196(54.1)$ & 85 / 148 (57.4) & 21 / 48 (43.8) & $54 / 54(100)$ * & $49 / 49(100) \ddagger$ & $5 / 5(100) \ddagger$ & $52 / 142(36.6)$ * & 36 / 99 (36.4) & $16 / 43(37.2) \ddagger$ \\
\hline SHANK2 & $16 / 196(8.2)$ & $15 / 148(10.1)$ & $1 / 48(2.1)$ & $13 / 52(25)^{*}$ & $13 / 47(27.7) \ddagger$ & $0 / 5(0)$ & $3 / 144(2.1)^{*}$ & $2 / 101(2)$ & $1 / 43(2.3)$ \\
\hline TPCN2 & $103 / 196(52.6)$ & $79 / 148(53.4)$ & $24 / 48(50)$ & $33 / 35(94.3)$ * & $31 / 33(93.9) \ddagger$ & $2 / 2(100)$ & $70 / 161(43.5)^{*}$ & $48 / 115(41.7)$ & 22 / 46 (47.8) \\
\hline \multirow[t]{2}{*}{ 11q13.4 Gene Expression } & Total & Total Smokers & Total Non-Smokers & Amplified & Amplified Smokers & Amplified Non-Smokers & Non-Amplified & $\begin{array}{l}\text { Non-Amplified } \\
\text { Smokers }\end{array}$ & $\begin{array}{l}\text { Non-Amplified } \\
\text { Non-Smokers } \\
\end{array}$ \\
\hline & OX / Amp (\%) & OX / Amp (\%) & OX/Amp (\%) & OX / Amp (\%) & OX / Amp (\%) & OX / Amp (\%) & OX / Amp (\%) & OX / Amp (\%) & OX / Amp (\%) \\
\hline \multicolumn{10}{|l|}{ Overexpressed } \\
\hline DHCR7 & $60 / 196$ (30.6) & $52 / 148(35.1)^{*}$ & $8 / 48(16.7)^{*}$ & $33 / 35(94.3)$ * & $31(100) \dagger \ddagger$ & $2(50)$ & $27 / 161(16.8)$ * & $21(17.95)$ & $6(13.64)$ \\
\hline NADSYN1 & 29 / 196 (14.8) & $25 / 148(16.9)$ & $4 / 48$ (8.3) & $26 / 35(74.3)$ * & $23(74.19)$ & $3(75) \ddagger$ & $3 / 161(1.9)$ * & $2(1.71)$ & $1(2.27) \ddagger$ \\
\hline RNF121 & $60 / 196(30.6)$ & $39 / 148(26.4)$ * & $21 / 48(43.8)$ * & $23 / 27(85.2)$ * & $21(87.5) \ddagger$ & $2(66.67)$ & $37 / 169(21.9)$ * & $18(14.52) \dagger$ & $19(42.22) \dagger$ \\
\hline \multirow[t]{2}{*}{ 9p21.3 Gene Expression } & Total & Total Smokers & Total Non-Smokers & Deleted & Deleted Smokers & Deleted Non-Smokers & Non-Deleted & $\begin{array}{l}\text { Non-Deleted } \\
\text { Smokers }\end{array}$ & $\begin{array}{l}\text { Non-Deleted Non } \\
\text { Smokers }\end{array}$ \\
\hline & UX / Del (\%) & UX / Del (\%) & UX / Del (\%) & UX / Del (\%) & UX / Del (\%) & UX / Del (\%) & UX / Del (\%) & OX / Amp (\%) & OX / Amp (\%) \\
\hline \multicolumn{10}{|l|}{ Underexpressed } \\
\hline C9ORF53 & $0 / 196(0)$ & $0 / 148(0)$ & $0 / 48(0)$ & $0 / 49(0)$ & $0(0) \ddagger$ & $0(0)$ & $0 / 147(0) *$ & $0(0)$ & $0(0)$ \\
\hline CDKN2A & $12 / 196(6.1)$ & $12 / 148(8.1)^{*}$ & $0 / 48(0)^{*}$ & $11 / 50(22)$ * & $11(25) \ddagger$ & $0(0)$ & $1 / 146(0.7)$ & $1(0.96)$ & $0(0)$ \\
\hline CDKN2B & $41 / 196$ (20.9) & $35 / 148$ (23.6) & $6 / 48(12.5)$ & $35 / 45(77.8)$ * & $30(76.92) \ddagger$ & $5(83.33) \ddagger$ & $6 / 151(4)^{*}$ & $5(4.59)$ & $1(2.38) \ddagger$ \\
\hline MTAP & $28 / 196(14.3)$ & $20 / 148(13.5)$ & $8 / 48(16.7)$ & $21 / 28(75) *$ & $16(69.57) \ddagger$ & $5(100) \ddagger$ & $7 / 168(4.2)$ * & $4(3.2)$ & $3(6.98) \ddagger$ \\
\hline \multirow[t]{2}{*}{ 9p24.1 Gene Expression } & Total & Total Smokers & Total Non-Smokers & Deleted & Deleted Smokers & Deleted Non-Smokers & Non-Deleted & $\begin{array}{l}\text { Non-Deleted } \\
\text { Smokers }\end{array}$ & $\begin{array}{l}\text { Non-Deleted Non } \\
\text { Smokers }\end{array}$ \\
\hline & UX / Del (\%) & UX / Del (\%) & UX / Del (\%) & UX / Del (\%) & UX / Del (\%) & UX / Del (\%) & UX / Del (\%) & OX / Amp (\%) & OX / Amp (\%) \\
\hline \multicolumn{10}{|l|}{ Underexpressed } \\
\hline PTPRD & $0 / 196(0)$ & $0 / 148(0)$ & $0 / 48(0)$ & $0 / 16(0)$ & $0(0)$ & $0(0)$ & $0 / 180(0)$ & $0(0)$ & $0(0)$ \\
\hline \multicolumn{10}{|c|}{${ }^{*} \mathrm{p}<0.05$ Total Amplified/Deleted vs Total Non-Amplified/Deleted or Total Smoker vs Total Non-Smoker by Pearson chi-squareed test (bottom) } \\
\hline \multicolumn{10}{|c|}{$\begin{array}{l}\dagger p<0.05 \text { Amplified/Deleted Smoker vs Amplified/Deleted Non-Smoker or Non-Amplified/Deleted Smoker vs Non-Amplified/Deleted Non-Smoker by Pearson chi-squared } \\
\ddagger p<0.05 \text { Amplified/Deleted Smoker vs Non-Amplified/Deleted Smoker or Amplified/Deleted Non-Smoker vs Non-Amplified/Deleted Non-Smoker by Pearson chi-squared }\end{array}$} \\
\hline \multicolumn{10}{|c|}{$\begin{array}{ll}\text { OX: Overexpressed; UX: Underexpressed; Amp: Amplified; Del: Deleted } & \\
\end{array}$} \\
\hline Smokers: Pack-year $>0$ or $\mathrm{P}$ & Patient Smoking H & -listory; Non-Smok & kers: Pack-year $=0$ or $r$ & no reported Patie & ient Smoking $\mathrm{Hi}$ & & & & \\
\hline
\end{tabular}

\section{Supplementary Table S4. Smoking-associated Gene Overexpression of HNSCC Patients by}

Smoking, 11q13.3 and 11q13.4 Cytoband Amplification Status. Related to Figure 4. 

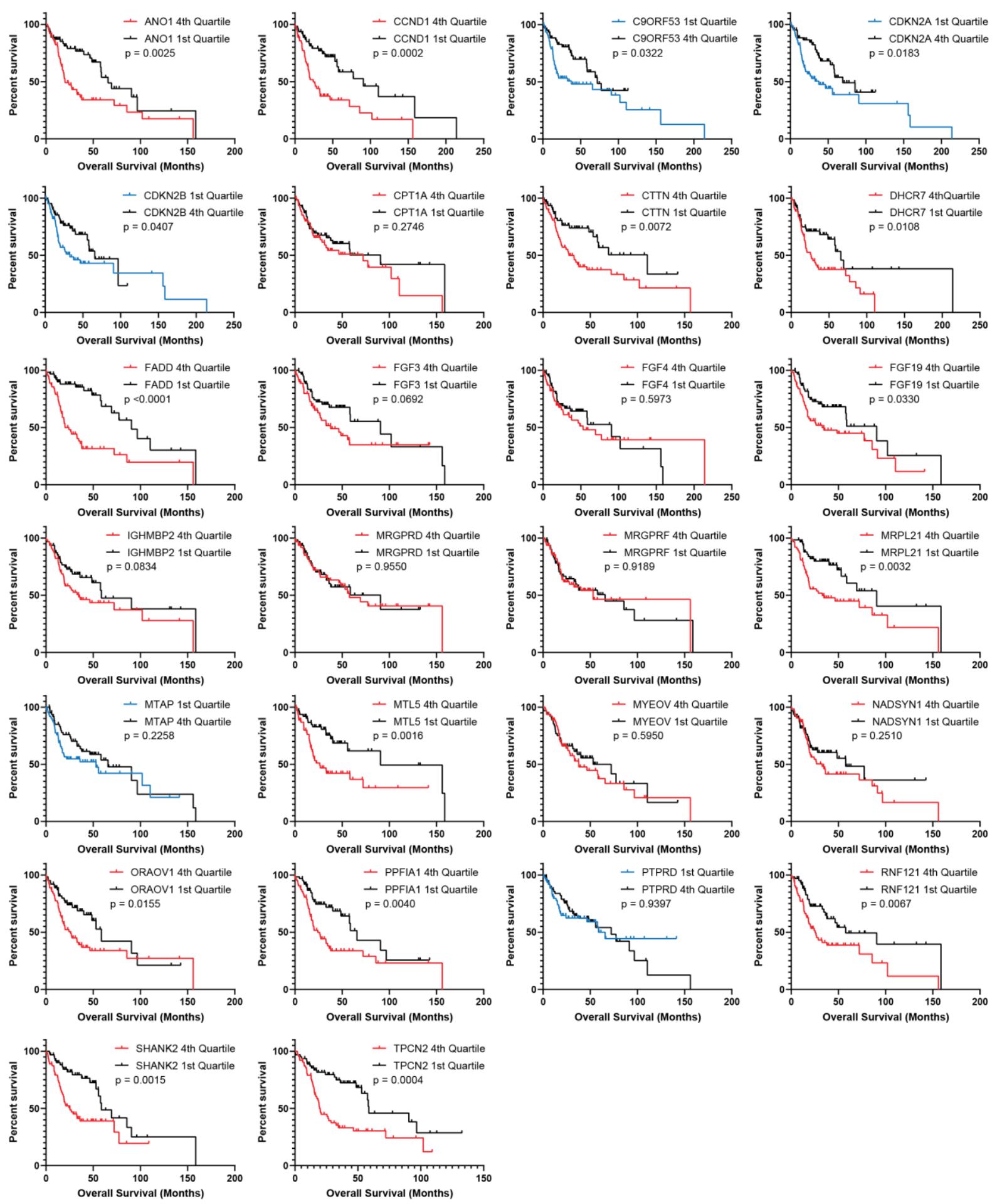

\section{Supplementary Figure S1. Determination of smoking-associated expression signature through}

effect of differential gene expression on overall survival. Overall Survival analysis of the differential expression levels for each identified gene comparing the first quartile (lowest $25 \%$ ) to the fourth quartile (highest $25 \%$ ). Genes with a significant effect on overall survival were combined into the smokingassociated expression signature. 


\section{SAES Gene Expression Effect on Overall Survival}

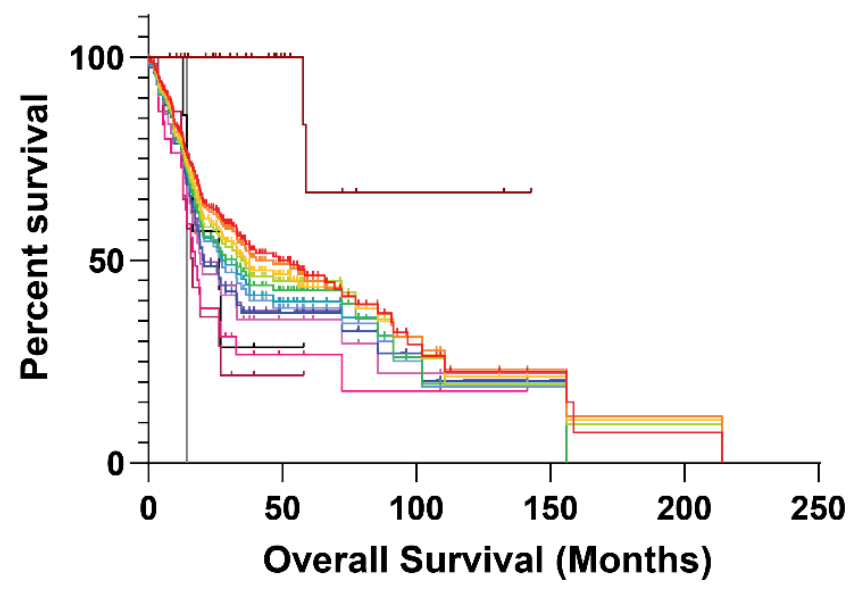

- 0 Signature Genes $\mid \mathrm{N}=25$ | Median = Undefined

- At Least 1 Signature Gene $|N=228|$ Median $=53.0$ months $\mid$ vs. $0 p=0.0007$ - At Least 2 Signature Genes $|N=210|$ Median $=40.1$ months $\mid$ vs. $0 p=0.0005$ - At Least 3 Signature Genes $|N=175|$ Median $=36.3$ months $\mid$ vs. $0 p=0.0003$ - At Least 4 Signature Genes $|N=153|$ Median $=34.6$ months $\mid$ vs. $0 p=0.0003$ - At Least 5 Signature Genes $|N=131|$ Median $=32.7$ months $\mid$ vs. $0 p=0.0002$ - At Least 6 Signature Genes $|N=111|$ Median $=27.4$ months $\mid$ vs. $0 p=0.0001$ - At Least 7 Signature Genes $|N=95|$ Median $=26.9$ months $\mid$ vs. $0 p<0.0001$ - At Least 8 Signature Genes $|N=78|$ Median $=20.2$ months $\mid$ vs. $0 p<0.0001$ - At Least 9 Signature Genes $|N=61|$ Median $=20.8$ months $\mid$ vs. $0 p<0.0001$ - At Least 10 Signature Genes $|N=43|$ Median $=19.2$ months $\mid$ vs. $0 p<0.0001$ - At Least 11 Signature Genes $|N=30|$ Median $=17.4$ months $\mid$ vs. $0 p<0.0001$ - At Least 12 Signature Genes $|N=15|$ Median $=16.4$ months $\mid$ vs. $0 p<0.0001$ - At Least 13 Signature Genes $|N=7|$ Median $=26.3$ months $\mid$ vs. $0 p<0.0001$ - At Least 14 Signature Genes $|N=1|$ Median $=14.2$ months $\mid$ vs. $0 p<0.0001$

\section{SAES Gene Expression Effect on Disease-Free Survival}

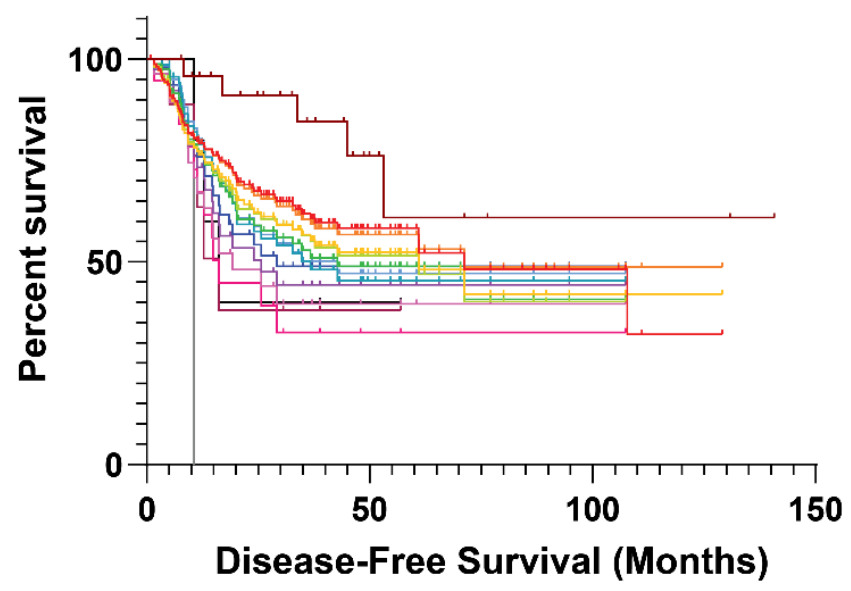

- 0 Signature Genes $|N=25|$ Median = Undefined

- At Least 1 Signature Gene $|\mathrm{N}=158|$ Median $=71.2$ months $\mid$ vs. $0 p=0.0527$ - At Least 2 Signature Genes $|N=144|$ Median $=71.2$ months $\mid$ vs. $0 p=0.0589$ - At Least 3 Signature Genes $|N=119|$ Median $=61.1$ months $\mid$ vs. $0 p=0.0263$ - At Least 4 Signature Genes $|N=102|$ Median $=61.1$ months $\mid$ vs. $0 p=0.0240$ - At Least 5 Signature Genes $|N=85|$ Median $=42.8$ months $\mid$ vs. $0 p=0.0187$ - At Least 6 Signature Genes $|N=71|$ Median $=36.3$ months $\mid$ vs. $0 p=0.0166$ - At Least 7 Signature Genes $|N=62|$ Median $=42.8$ months $\mid$ vs. $0 p=0.0236$ - At Least 8 Signature Genes $|N=49|$ Median $=29.2$ months $\mid$ vs. $0 p=0.0217$ - At Least 9 Signature Genes $|N=39|$ Median $=25.7$ months $\mid$ vs. $0 p=0.0102$ - At Least 10 Signature Genes $|N=28|$ Median $=19.2$ months $\mid$ vs. $0 p=0.0041$ - At Least 11 Signature Genes $|N=19|$ Median $=16.2$ months $\mid$ vs. $0 p=0.0013$ - At Least 12 Signature Genes $|N=9|$ Median $=16.2$ months $\mid v s .0 p=0.0084$ - At Least 13 Signature Genes $|N=5|$ Median $=16.2$ months $\mid$ vs. $0 p=0.0546$ - At Least 14 Signature Genes $|N=1|$ Median $=10.6$ months $\mid$ vs. $0 p=0.0012$

\section{Supplementary Figure S2. Additive effect of SAES gene over- or underexpression on overall}

survival. Kaplan-Meier survival analysis comparing the effect of additional over- or underexpression of a SAES genes on overall (top) and disease-free (bottom) survival. Median survival and p-value for each signature are color-key on the right. 
Gene Copy-Number Alteration

Gene Expression

\begin{tabular}{|c|c|c|c|c|c|c|c|c|}
\hline & Cox Hazard Ratio & $2.5 \% \mathrm{Cl}$ & $97.5 \% \mathrm{Cl}$ & P-value & Cox Hazard Ratio & $2.5 \% \mathrm{Cl}$ & $97.5 \% \mathrm{Cl}$ & P-value \\
\hline ANO1 & 1.050 & 0.940 & 1.174 & 0.386 & 1.298 & 1.088 & 1.549 & 0.004 \\
\hline C9orf53 & 0.688 & 0.493 & 0.959 & 0.027 & 0.971 & 0.933 & 1.010 & 0.142 \\
\hline CCND1 & 1.045 & 0.928 & 1.177 & 0.463 & 1.122 & 1.003 & 1.255 & 0.043 \\
\hline CDKN2A & 0.705 & 0.507 & 0.981 & 0.038 & 0.977 & 0.895 & 1.066 & 0.605 \\
\hline CDKN2B & 0.836 & 0.601 & 1.162 & 0.286 & 0.917 & 0.797 & 1.054 & 0.222 \\
\hline CPT1A & 0.975 & 0.824 & 1.154 & 0.770 & 1.043 & 0.937 & 1.160 & 0.443 \\
\hline CTTN & 1.043 & 0.934 & 1.164 & 0.455 & 1.109 & 1.018 & 1.207 & 0.017 \\
\hline DHCR7 & 1.125 & 0.973 & 1.300 & 0.112 & 1.117 & 0.998 & 1.250 & 0.054 \\
\hline FADD & 1.048 & 0.938 & 1.171 & 0.404 & 1.100 & 1.025 & 1.181 & 0.008 \\
\hline FGF19 & 1.060 & 0.942 & 1.192 & 0.332 & 1.001 & 0.999 & 1.004 & 0.290 \\
\hline FGF3 & 1.074 & 0.956 & 1.207 & 0.227 & 0.996 & 0.980 & 1.013 & 0.653 \\
\hline FGF4 & 1.071 & 0.952 & 1.204 & 0.254 & 0.996 & 0.980 & 1.014 & 0.679 \\
\hline IGHMBP2 & 1.019 & 0.871 & 1.191 & 0.818 & 1.040 & 0.982 & 1.102 & 0.178 \\
\hline MRGPRD & 1.058 & 0.912 & 1.227 & 0.459 & 0.994 & 0.940 & 1.051 & 0.823 \\
\hline MRGPRF & 1.040 & 0.897 & 1.205 & 0.604 & 0.848 & 0.657 & 1.093 & 0.203 \\
\hline MRPL21 & 1.004 & 0.856 & 1.177 & 0.963 & 1.063 & 1.016 & 1.112 & 0.009 \\
\hline MTAP & 0.978 & 0.719 & 1.329 & 0.885 & 0.936 & 0.848 & 1.034 & 0.193 \\
\hline MTL5 & 0.983 & 0.816 & 1.184 & 0.855 & 1.046 & 0.960 & 1.139 & 0.304 \\
\hline MYEOV & 1.081 & 0.957 & 1.221 & 0.211 & 1.148 & 0.919 & 1.433 & 0.223 \\
\hline NADSYN1 & 1.125 & 0.973 & 1.300 & 0.112 & 1.037 & 0.881 & 1.220 & 0.664 \\
\hline ORAOV1 & 1.048 & 0.931 & 1.180 & 0.438 & 1.030 & 0.980 & 1.083 & 0.245 \\
\hline PPFIA1 & 1.045 & 0.937 & 1.167 & 0.428 & 1.053 & 0.995 & 1.113 & 0.073 \\
\hline PTPRD & 1.121 & 0.921 & 1.364 & 0.254 & 0.964 & 0.890 & 1.044 & 0.364 \\
\hline RNF121 & 1.238 & 1.012 & 1.515 & 0.038 & 1.079 & 0.951 & 1.223 & 0.238 \\
\hline SHANK2 & 1.028 & 0.919 & 1.150 & 0.625 & 1.199 & 0.964 & 1.491 & 0.102 \\
\hline TPCN2 & 1.040 & 0.903 & 1.197 & 0.586 & 1.111 & 1.026 & 1.203 & 0.010 \\
\hline DEFB108B & 1.129 & 0.928 & 1.373 & 0.224 & & & & \\
\hline ENPP7P8 & 1.090 & 0.910 & 1.306 & 0.350 & & & & \\
\hline FAM86C1 & 1.121 & 0.919 & 1.368 & 0.259 & & & & \\
\hline MIR3164 & 1.040 & 0.903 & 1.197 & 0.586 & & & & \\
\hline MIR3664 & 1.105 & 0.977 & 1.249 & 0.114 & & & & \\
\hline MIR548K & 1.046 & 0.937 & 1.169 & 0.423 & & & & \\
\hline RNA5SP342 & 1.129 & 0.928 & 1.373 & 0.224 & & & & \\
\hline UNC93B6 & 1.177 & 1.008 & 1.374 & 0.039 & & & & \\
\hline ZNF705E & 1.129 & 0.928 & 1.373 & 0.224 & & & & \\
\hline
\end{tabular}

Supplementary Table S5. Multivariate Analysis Predicting Overall Survival using Age, Sex, AJCC Stage, HPV-Status, Extracapsular Spread, Perineural Invasion, and Lymphovascular Invasion 


\section{Relative Risk of Death Associated with SAES Gene Expression}
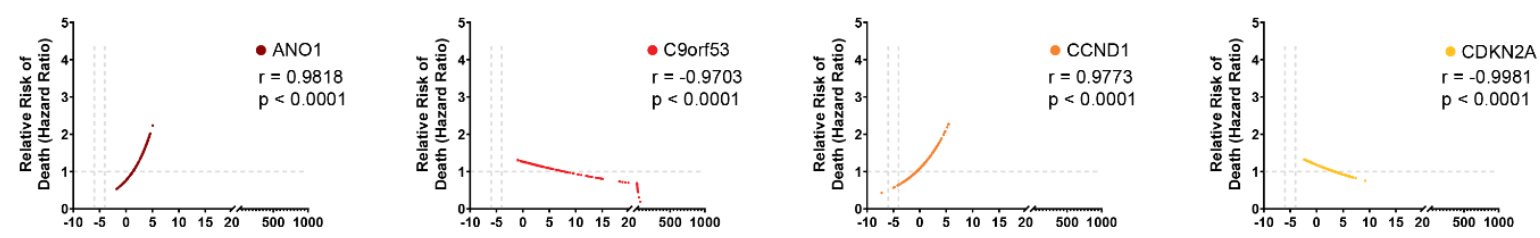

Standardized Gene Expression (z-score)
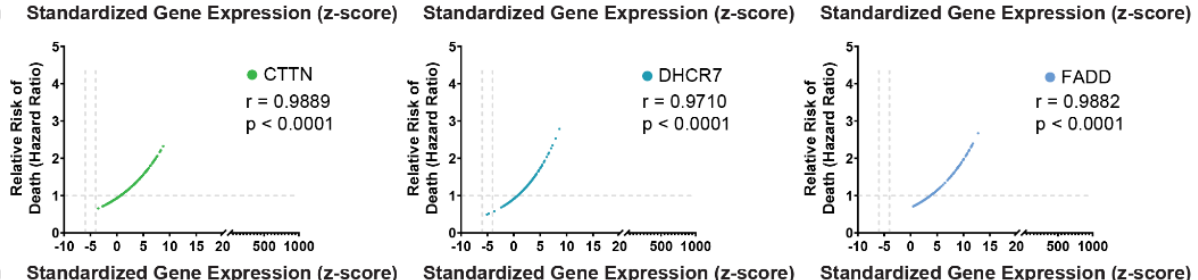

Standardized Gene Expression (z-score)
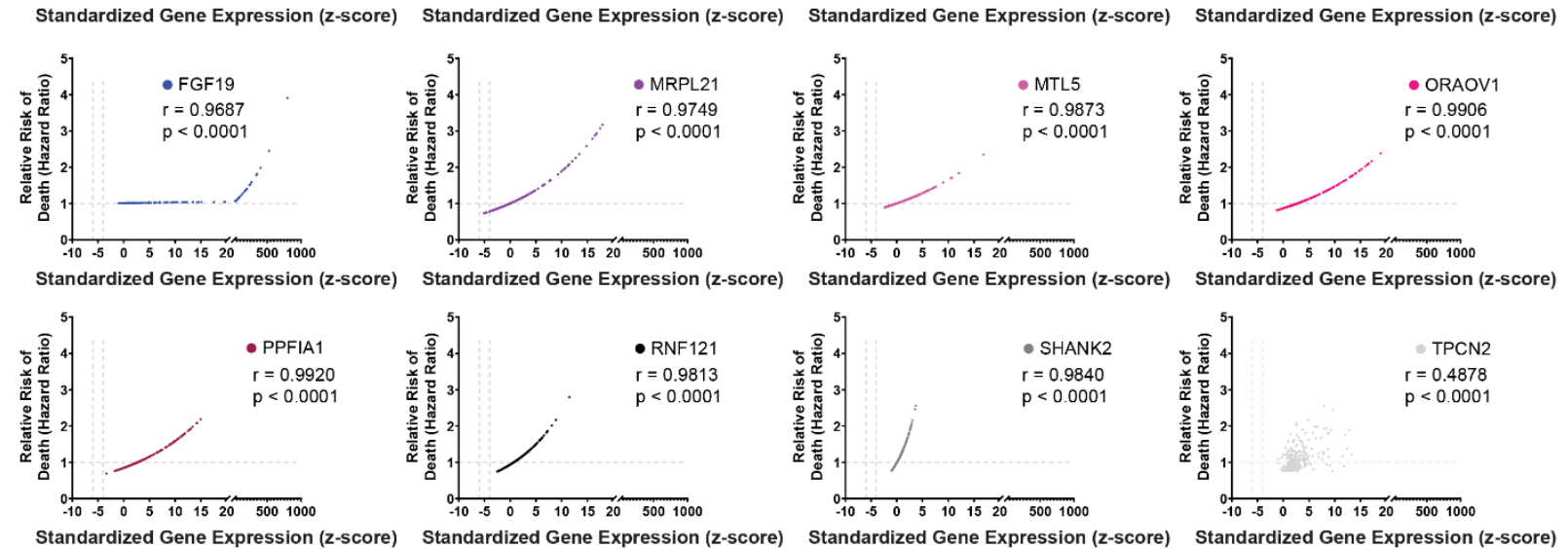

Standardized Gene Expression (z-score)

Standardized Gene Expression (z-scor

Standardized Gene Expression (z-score)

Standardized Gene Expression (z-score)

\section{Supplementary Figure S3. Relative risk of death of each smoking-associated expression}

signature gene. Pearson correlations between standardized gene expression (z-score) and risk of death

(hazard ratio) for each individual SAES gene in Fig. 5C 


\begin{tabular}{|c|c|c|c|c|}
\hline & odds ratio & $2.5 \% \mathrm{Cl}$ & $97.5 \% \mathrm{Cl}$ & P-value \\
\hline MTL5 & 1.013 & \begin{tabular}{|l|}
1.005 \\
\end{tabular} & 1.020 & 8.05E-04 \\
\hline CPT1A & 1.013 & 1.006 & 1.020 & 6.07E-04 \\
\hline MRPL21 & 1.013 & 1.006 & 1.021 & 2.69E-04 \\
\hline IGHMBP2 & 1.013 & 1.006 & 1.021 & $2.75 \mathrm{E}-04$ \\
\hline MRGPRD & 1.016 & 1.009 & 1.024 & 2.57E-05 \\
\hline MRGPRF & 1.017 & 1.010 & 1.025 & $7.16 \mathrm{E}-06$ \\
\hline TPCN2 & 1.017 & 1.009 & 1.024 & $1.24 \mathrm{E}-05$ \\
\hline MIR3164 & 1.017 & 1.009 & 1.024 & $1.24 \mathrm{E}-05$ \\
\hline MYEOV & 1.017 & 1.010 & 1.024 & 4.16E-06 \\
\hline CCND1 & 1.016 & 1.010 & 1.024 & $3.92 \mathrm{E}-06$ \\
\hline ORAOV1 & 1.016 & 1.010 & 1.024 & $3.92 \mathrm{E}-06$ \\
\hline FGF19 & 1.016 & 1.010 & 1.024 & $3.92 \mathrm{E}-06$ \\
\hline FGF4 & 1.016 & 1.010 & 1.024 & $3.92 \mathrm{E}-06$ \\
\hline FGF3 & 1.016 & 1.009 & 1.023 & $6.15 \mathrm{E}-06$ \\
\hline ANO1 & 1.017 & 1.011 & 1.025 & $1.37 \mathrm{E}-06$ \\
\hline FADD & 1.017 & 1.010 & 1.024 & $2.20 \mathrm{E}-06$ \\
\hline PPFIA1 & 1.017 & 1.010 & 1.024 & $2.42 \mathrm{E}-06$ \\
\hline MIR548K & 1.017 & 1.010 & 1.024 & $2.42 \mathrm{E}-06$ \\
\hline CTTN & 1.016 & 1.010 & 1.024 & $3.36 \mathrm{E}-06$ \\
\hline SHANK2 & 1.016 & 1.009 & 1.023 & $6.12 \mathrm{E}-06$ \\
\hline MIR3664 & 1.015 & 1.008 & 1.022 & $3.13 E-05$ \\
\hline DHCR7 & 1.016 & 1.009 & 1.024 & $2.46 \mathrm{E}-05$ \\
\hline NADSYN1 & 1.016 & 1.009 & 1.024 & $2.46 \mathrm{E}-05$ \\
\hline UNC93B6 & 1.017 & 1.010 & 1.025 & $1.47 \mathrm{E}-05$ \\
\hline ENPP7P8 & 1.016 & 1.009 & 1.025 & 4.12E-05 \\
\hline FAM86C1 & 1.016 & 1.009 & 1.025 & 7.30E-05 \\
\hline ZNF705E & 1.015 & 1.008 & 1.023 & $1.43 \mathrm{E}-04$ \\
\hline DEFB108B & 1.015 & 1.008 & 1.024 & $1.25 \mathrm{E}-04$ \\
\hline RNA5SP342 & 1.015 & 1.008 & 1.024 & $1.25 \mathrm{E}-04$ \\
\hline RNF121 & 1.015 & 1.008 & 1.024 & $1.51 \mathrm{E}-04$ \\
\hline MTAP & 1.004 & 0.998 & 1.010 & 0.177 \\
\hline C9orf53 & 1.003 & 0.997 & 1.009 & 0.294 \\
\hline CDKN2A & 1.003 & 0.997 & 1.008 & 0.362 \\
\hline CDKN2B & 1.003 & 0.997 & 1.009 & 0.266 \\
\hline PTPRD & 1.001 & 0.992 & 1.008 & 0.822 \\
\hline
\end{tabular}

Supplementary Table S6. Predictive Probability Linear Regression of Gene CNA by GISTIC2 Value and Pack-Year. Related to Figure 5. 


\begin{tabular}{|c|c|c|c|c|}
\hline & Estimate & Standard Error & z.value & P.value \\
\hline ANO1 & 0.006343591 & 0.004242823 & \begin{tabular}{|l|l|}
3 & 1.495134 \\
\end{tabular} & 0.135 \\
\hline CCND1 & 0.012633468 & 0.005410455 & 2.33501 & $0.02 C$ \\
\hline CPT1A & 0.013633059 & 0.005939486 & 2.295327 & 0.022 \\
\hline CTTN & 0.017000566 & 0.004766874 & 3.566397 & 0.000 \\
\hline DHCR7 & 0.021053314 & 0.004994869 & 4.214988 & 0.000 \\
\hline FADD & 0.01377474 & 0.005896312 & 2.336162 & 0.019 \\
\hline FGF19 & 0.011723851 & 0.004431076 & 2.645825 & 0.008 \\
\hline FGF3 & 0.021877574 & 0.007099945 & 3.081372 & 0.002 \\
\hline FGF4 & -0.000668514 & 0.007599246 & \begin{tabular}{|l|l|}
5 & -0.087971 \\
\end{tabular} & 0.930 \\
\hline IGHMBP2 & 0.006795303 & 0.004361989 & \begin{tabular}{|l|l|}
9 & 1.557845 \\
\end{tabular} & 0.119 \\
\hline MRGPRD & 0.00612869 & 0.004965211 & 1.234326 & 0.217 \\
\hline MRGPRF & 0.002650902 & 0.009437755 & 0.280883 & 0.779 \\
\hline MRPL21 & 0.010992611 & 0.004682154 & 2.347768 & 0.019 \\
\hline MTL5 & 0.015144763 & 0.004639637 & 3.264213 & 0.001 \\
\hline MYEOV & 0.018813873 & 0.010098192 & 1.863093 & 0.062 \\
\hline NADSYN1 & 0.021029108 & 0.005666638 & 3.711038 & 0.000 \\
\hline ORAOV1 & 0.009501594 & 0.004406822 & 2.15611 & 0.031 \\
\hline PPFIA1 & 0.015513346 & 0.004739885 & 3.272937 & 0.001 \\
\hline RNF121 & -0.002647988 & 0.004567647 & -0.579727 & 0.562 \\
\hline SHANK2 & 0.014984733 & 0.00651749 & 2.299157 & 0.021 \\
\hline TPCN2 & 0.006648249 & 0.004262356 & 1.559759 & 0.119 \\
\hline C9orf53 & \multicolumn{4}{|c|}{$*$ all values are 0 in C9orf53 } \\
\hline CDKN2A & $1.00 \mathrm{E}-02$ & $7.51 \mathrm{E}-03$ & $1.33 \mathrm{E}+00$ & 0.183 \\
\hline CDKN2B & $5.53 \mathrm{E}-03$ & $4.86 \mathrm{E}-03$ & $1.14 \mathrm{E}+00$ & 0.255 \\
\hline MTAPP & $3.41 \mathrm{E}-03$ & $5.66 \mathrm{E}-03$ & $6.03 \mathrm{E}-01$ & 0.547 \\
\hline PTPRD & \multicolumn{4}{|c|}{$*$ all values are 0 in PTPRD } \\
\hline
\end{tabular}

Supplementary Table S7. Predictive Probability Linear Regression of Gene Over- or Underexpression by Z-score and Pack-Year. Related to Figure 5. 


\section{Study 3: The CCND1 3'-UTR as a Potential Driver of Poor Outcome in Chromosome 11q13-amplified Head and Neck Squamous Cell Carcinoma}

Brenen W. Papenberg ${ }^{1}$, Ivan Martinez ${ }^{2}$, Peter Stoilov ${ }^{1}$ and Scott A. Weed ${ }^{1 *}$

Departments of ${ }^{1}$ Biochemistry, ${ }^{2}$ Microbiology, Immunology and Cell Biology, Program in Cancer Cell Biology, West Virginia University, Morgantown, West Virginia, 26506

Running title: CCND1 3'-UTR in HNSCC progression

Corresponding Author: "Scott A. Weed, West Virginia University Cancer Institute, P.O. Box 9300, Morgantown, WV 26506, Phone: 304-293-3016, Fax: 304-293-4667, scweed@hsc.wvu.edu.

Keywords: CCND1, competing endogenous RNA, 3'-UTR, microRNA, head and neck cancer 


\section{Abstract}

Background: Three prime untranslated regions (3'-UTRs) of messenger (m)RNAs can serve as competing endogenous (ce)RNAs through the binding and titration of regulatory micro (mi)RNAs. Inactivation of tumorsuppressive miRNAs by UTRs with shared miRNA response elements (MREs) promotes cancer progression through permissive expression of miRNA-targeted oncogenic transcripts. Chromosome 11q13 amplification drives the progression of head and neck squamous cell carcinoma (HNSCC) and other cancer types through oncogenic protein overexpression. However, the collective ability of 3'-UTR regions in 11 q13 overexpressed transcripts to function as oncogenic ceRNAs is unclear.

Methods: We utilized TargetScan to predict MREs for the 3'-UTR of 11q13 genes and The Cancer Genome Atlas (TCGA) to analyze and stratify gene, exon, and protein expression values. Western blotting was used to determine protein expression. mRNA in-situ hybridization (mRNA-ISH) was used to quantify mRNA expression and localization in HNSCC cell lines.

Results: Six transcripts from the most commonly amplified 11q13 genes in HNSCC are overexpressed and maintain the 3'-UTR. Of these, the CCND1 3'-UTR is predicted to bind 31 tumor suppressive miRNAs, thus has oncogenic ceRNA characteristics. Overexpression of the 3'-UTR encoded from CCND1 exon 5 denotes worse clinical outcome. HNSCC cells lines with 11q13 amplification overexpress the CCND1 3'-UTR in the cytoplasm, where it can function as a ceRNA. Correlation analysis of global protein expression in patients with CCND1 3'UTR overexpression identified significant protein overexpression from genes with 3'-UTR MREs homologous with CCND1. Four proteins (BID, RB1, PXN, MACC1) were positively correlated in human papillomavirus (HPV)positive, and eight proteins (SERPINE1, TYMS, DVL3, ITGA2, BCL2L11, CCNB1, GATA3, and E2F1) were positively correlated in HPV-negative HNSCC. Similar correlation analysis of four other 11q13-amplified cancer types identified SERPINE1, GATA3 and ITGA2 as common upregulated proteins in tumors with CCND1 3'-UTR overexpression, suggesting conservation of the potential CCND1 3'-UTR ceRNA mechanism.

Conclusions: An integrated approach across multiple patient cohorts in different cancers identified several candidate genes potentially regulated by high CCND1 3'-UTR overexpression and ceRNA activity, which may be utilized for additional therapeutic strategies to improve the outcomes of patients with CCND1 amplification and protein overexpression. 


\section{Background}

HNSCC is an aggressive neoplasm of the upper aerodigestive tract caused by tobacco, alcohol, and high-risk HPV infection(1-3). HPV-negative patients have a worse outcome than HPV-positive patients, stratifying the disease into distinct prognostic subtypes(4-6). TCGA and others have identified large-scale genomic copy number alterations (CNAs) in $\operatorname{HNSCC}(7,8)$. Of these, amplification of the $11 \mathrm{q} 13$ chromosome region occurs in $25-50 \%$ of cases and is the most frequent CNA in HPV-negative HNSCC $(9,10)$. Patients with $11 q 13$ amplification have more metastatic disease, shorter time to recurrence and die sooner than HPV-negative patients lacking amplification(11,12). The overexpressed G1/S cell cycle promoting protein cyclin D1, encoded from the CCND1 gene, is accepted as the main oncogenic driver in 11 q13 amplified cancer through increased cyclin-dependent kinase (CDK)4/6 activation and inhibition of the tumor suppressing retinoblastoma protein (pRB)(12-15). CCND1 amplification and protein overexpression are correlated with decreased patient survival and increased progression in HNSCC(16-19).

Current understanding of 11q13 amplification in mediating HNSCC progression is largely based on the study of protein products from amplified genes $(20,21)$. However, the role of the noncoding regions of overexpressed mRNA transcripts from any 11q13 amplicon gene (e.g., 5' and 3'-UTR regions) has not been evaluated. Noncoding UTR regions are known to contribute to cancer progression(22,23). Such transcripts can function as ceRNAs that operate via several mechanisms(24-27). One mechanism is titration of miRNAs through competitive binding of miRNAs shared with different transcripts containing homologous MREs(26,28,29). Binding of miRNAs to transcripts results in transcript degradation, or blocks translation without substantial degradation(30,31). Both mechanisms reduce protein expression from shared MRE-containing transcripts. Many studies in cancer have shown oncogenic ceRNA effects by long non-coding (Inc) pseudogenes and nonshortened 3'-UTR regions(16,29,32-36). Oncogenic ceRNAs thus sequester tumor-suppressive miRNAs at MREs shared with oncogenic target transcripts. This reduces the available levels of tumor-suppressive miRNAs, allowing transcripts normally degraded/blocked to persist and translate proteins(37). The ultimate result of such ceRNA activity is relief of tumor suppression that enhances neoplastic progression $(29,38)$.

Global ceRNA networks exist in $\operatorname{HNSCC}(39,40)$, where dysregulation of miRNA networks causes neoplastic gene expression(41-44). Such studies infer that the non-coding regions in 11q13 amplified transcripts may contribute to the aggressive advancement of HNSCC as ceRNAs. 11 q13 genes can be amplified up to 80-fold 
in HNSCC, increasing transcript levels $16-48$ times above baseline $(9,13,17,45)$. As such, highly overexpressed transcripts from the 11q13 amplicon may serve as ceRNAs in driving HNSCC. In this report we have evaluated the predicted MREs in the 3'-UTR regions of the most commonly overexpressed protein-coding genes from the 11 13 amplicon. The CCND1 3'-UTR region was the only overexpressed transcript containing a higher number of tumor suppressive MREs. Patients with CCND1 3'-UTR overexpression have worse overall survival. The CCND1 3'-UTR localizes to the cytoplasm in 11q13 amplified HNSCC cells, allowing it to serve as a ceRNA. Correlation analysis of protein expression from transcripts with MREs shared with the CCND1 3'-UTR identified four proteins with a positive correlation in HPV-positive HNSCC (BID, RB1, PXN, and MACC1) and eight positively correlated in HPV-negative HNSCC (SERPINE1, TYMS, DVL3, ITGA2, BCL2L11, CCNB1, GATA3, and E2F1) in patients with CCND1 3'-UTR overexpression. Overexpression of several of these proteins is also positively correlated with CCND1 3'-UTR overexpression in other cancers with 11 1913 amplification. This informatics-based approach provides new candidate protein and miRNA targets for further mechanistic interrogation of the underlying causes of poor clinical outcomes in 11q13 amplified cancers. 


\section{Methods}

\section{Clinical and Survival Data}

Clinical and survival data were obtained from the 2016_01_28 TCGA head-neck squamous cell carcinoma (TCGA-HNSC) cohort, downloaded from Broad Genomic Data Analysis Center (GDAC) Firehose(46). Data were retrieved from the level 4 TCGA data file All_CDEs.txt and cBioPortal(47). TCGA-HNSC consists of a total of 529 patients. However, only 263 patients had RNAseq data and were used for determining survival analyses. Patients were also evaluated for HPV in the All_CDEs.txt file, denoted by variable "hpv_status", which was used to stratify the cohort into confirmed HPV-positive and HPV-negative subsets. Kaplan-Meier P-values were calculated using Mantel-Cox log-rank test with an alpha of 0.05 .

\section{Determination of MiRNA Response Elements and Genetic Characteristics}

We used the University of California Santa Cruz (UCSC) Genome browser(48) and National Center for Biotechnology Information (NCBI) AceView(49) to determine lengths of open reading frames (ORFs), 3'-UTRs, exons, and introns. Predicted MREs for each gene were downloaded from TargetScan using the Conserved site context++ scores file(50). Genes from the $11 q 13$ amplicon were extracted and individual miRNAs were categorized as tumor-suppressive, oncogenic, or indeterminate using OncomiRDB(51). Additional miRNAs were identified by manual evaluation of the literature.

\section{Copy Number and Expression Analysis of Genes, Exons, and Proteins}

CNAs were determined by Genomic Identification of Significant Targets in Cancer (GISTIC)2(52) analysis of TCGA 2016_01_28 level 4 data for 24,776 genes(46). Briefly, raw GISTIC2 values within all_data_by_genex.txt were used to categorize patients by amplification (more than two extra copies), gene gain (two extra copies), homozygous deletion, or heterozygous deletion. Expression levels for genes were obtained from TCGA-HNSC ( $N=263)$, TCGA bladder urothelial carcinoma (TCGA-BLCA) $(\mathrm{N}=56)$, TCGA breast invasive carcinoma (TCGABRCA) ( $\mathrm{N}=791)$, TCGA esophageal carcinoma (TCGA-ESCA) ( $\mathrm{N}=185)$, and TCGA lung squamous cell carcinoma (TCGA-LUSC) ( $\mathrm{N=223)}$ 2016_01_28 level 3 RNAseq gene expression datasets(46). Expression levels for exons were obtained from TCGA-HNSC 2016_01_28 level 3 RNAseq exon expression dataset(46). Gene and exon analyses were conducted using log2 fold change (log2FC) normalization calculated from reads per kilobase of transcript, per million reads (RPKM) in normal adjacent tissues as baseline. High and low 
expression of each gene or exon was assessed by comparing the first quartile (lowest 25\%) of expression to the fourth quartile (highest $25 \%$ ). Changes in global mRNA expression were determined using the log2FC ratio between high and low CCND1 expression levels. Protein expression levels were obtained from TCGA-HNSC ( $\mathrm{N}=179)$, TCGA-BLCA ( $\mathrm{N}=50)$, TCGA-BRCA ( $\mathrm{N}=630)$, TCGA-ESCA ( $\mathrm{N}=126)$, and TCGA-LUSC ( $\mathrm{N}=149)$ 2016_01_28 level 3 RPPA AnnotateWithGene dataset(46). Protein expression analysis was conducted using the provided normalized values. Statistical significance was determined using Pearson correlation or one-way analysis of variance (ANOVA) and Tukey's post-hoc with an alpha of 0.05 .

\section{Western Blotting and Antibodies}

Western blotting was conducted as described(53) and visualized with autoradiography film (E3012, Denville Scientific) or captured by an Amersham Imager 600 (GE Healthcare Bio-Sciences). Antibodies used were anticyclin D1 (sc-718, 1:1000; Santa Cruz Biotechnology) and anti- $\beta$-actin (\#8457, 1:1000; Cell Signaling Technology).

\section{Cell Lines and Cell Culture}

Cell lines used were UMSCC19 and MSK921(54), MDA1586(55), UPCI:SCC040 and UPCI:SCC136(56) and HaCaT(57). UMSCC47(58) was purchased from the American Type Culture Collection (ATCC). All lines were propagated in Dulbecco's Modified Eagle Media (DMEM) supplemented with $10 \%$ fetal bovine serum and $1 \%$ penicillin-streptomycin.

\section{RNA In-Situ Hybridization}

RNA in-situ hybridization was performed as previously described(59). Briefly, custom Stellaris® FISH Probes were designed against CCND1 3'UTR by utilizing the Stellaris ${ }^{\circledR}$ RNA FISH Probe Designer (Biosearch Technologies, Inc., Petaluma, CA) available online at www.biosearchtech.com/stellarisdesigner (Version 2017). The CCND1 3'UTR were hybridized with Cy5. Stellaris RNA FISH Probe set labeled with (Biosearch Technologies, Inc.), following the manufacturer's instructions available online at www.biosearchtech.com/stellarisprotocolscells. Cells were grown on coverglass, permeabilized, and subsequently hybridized with a buffer containing custom fluorescent probes. Cells were then DAPI stained to visualize nuclei and mounted onto slides for microscopic analysis using a Nikon A1R confocal microscope. 
Corrected total cell fluorescence was then calculated for each cell line. Statistical significance was determined one-way ANOVA and Tukey's post-hoc with an alpha of 0.05 .

\section{Determination of CCND1-targeting miRNAs}

To determine genes that share MREs for CCND1-targeting miRNAs to compare global and per-gene expression changes, the TargetScan Predicted Targets (default predictions) file was downloaded(50). CCND1 was extracted and miRNAs predicted to bind the 3'-UTR were used to find other genes with shared miRNA. Genes that are targeted by any shared miRNA are referred to as "targets". Genes that are not targeted by any of the predicted CCND1-targeting miRNA are "controls."

\section{Survival Analyses}

Survival was assessed using overall survival months and vital status supplied from TCGA-HNSC, TCGA-BLCA, TCGA-BRCA, TCGA-ESCA, and TCGA-LUSC clinical datasets. Data were stratified by applicable parameters and graphed with the Kaplan-Meier method. Statistical significance was determined using Mantel-Cox log-rank test with an alpha of 0.05 . 


\section{Results}

\section{The 11 q13 amplification is associated with overexpressed transcripts that harbor tumor-suppressing}

miRNA response elements

Based on previously-defined subdivisions in breast cancer(20), the 11q13 amplicon was analyzed for amplification frequency found within TCGA-HNSC patients. The highest frequency of amplification was seen in core 2, with all genes being amplified in more than $20 \%$ of patients (Figure $1 \mathrm{~A}$ ). The protein-coding genes within this region were evaluated for 3'-UTR length and expression of the exon containing the 3'-UTR within TCGAHNSC patients. Several amplified genes contain long 3'-UTRs and displayed 3'-UTR overexpression. These include CCND1, ORAOV1, ANO1, FADD, PPFIA1, and CTTN in greater than $10 \%$ of patients (Figure 1B). To determine if these long 3'-UTRs harbored MREs for tumor-suppressing miRNAs, TargetScan Conserved site context++ scores were used to predict MREs and miRNA were classified as tumor-suppressive, oncogenic, or undetermined. Four 11q13 genes had over ten predicted tumor-suppressive MREs: CCND1 (N=50), ANO1 $(\mathrm{N}=11)$, PPFIA1 $(\mathrm{N}=13)$, and SHANK2 $(\mathrm{N}=47)$ (Figure 1C). CCND1 was selected for further evaluation due to the unusually high amount of predicted tumor suppressive MREs in the 3'-UTR, its high tumor-suppressive miR:oncomiR ratio (Supplementary Figure S1), and because the 3'-UTR is overexpressed in HNSCC. Several well-known tumor-suppressive miRNAs are predicted to bind to the CCND1 3'-UTR, including miR-1-3p, miR16-5p, miR-17-5p, and let-7-5p (Figure 1D). The potential ability to bind and sequester a wide variety of tumorsuppressing miRNAs could contribute to the poor survival seen in patients with genomic CCND1 amplification (Figure 1E).

\section{CCND1 3'-UTR is overexpressed in 11q13 the cytosol of amplified cells and is associated with poor}

\section{patient survival}

The CCND1 transcript is encoded from five exons, with exon five containing the last 165nts of the ORF and the 3,192nts 3'-UTR (Figure 2A). After normalizing exon RPKM expression values, all five exons are expressed in their entirety in HNSCC patients. After stratifying patients by amplification (two or more extra copies), gene gain (two extra copies), euploid (no extra copies), heterozygous deletion (loss of one copy), or homozygous deletion

(loss of both copies), CCND1 expression is highest in amplified patients, where amplification and gene gain events exhibit elevated expression compared to euploid patients (Figure 2B). When comparing overall survival 
for HNSCC patients with high exon 5 expression (highest quarter) to patients with low exon 5 expression (lowest quarter), patients with high exon 5 expression have decreased median (23.3 vs 90.6 months) and 5-year (32.7\% vs $55.7 \%$ ) overall survival (Figure $2 \mathrm{C}$ ).

Since nuclear export of the CCND1 transcript is required for oncogenic transformation(60), the ability of the 3'UTR to function as a ceRNA likely occurs in the cytoplasm. To determine the localization of the 3'-UTR in 11q13amplified HNSCC, we evaluated the expression level and subcellular localization of the CCND1 transcript in HNSCC cell lines. As expected, cyclin D1 protein overexpression was observed in HNSCC cell lines containing 11 13 amplification compared to lines lacking amplification (Figure 3A). To measure CCND1 3'-UTR levels in these lines, we performed mRNA-ISH using probes homologous to sequences in the 3'-UTR. 11q13 amplified HNSCC lines contained 2-4-fold higher CCND1 3'-UTR levels compared to non-amplified cell lines (Figure 3B). Confocal microscopic evaluation of mRNA-ISH lines determined that the CCND1 3'-UTR is largely enriched in the cytosol of $11 q 13$ amplified cells (Figure 3C), supporting a role for a cytoplasmic ceRNA function, as well as confirming that the 3'-UTR is not truncated when overexpressed in HNSCC.

\section{Target RNAs sharing CCND1 MREs are not globally altered by CCND1 3'-UTR overexpression}

To determine if CCND1 3'-UTR overexpression exerts a global ceRNA effect on mRNA expression, we utilized TargetScan predicted targets as well as TCGA mRNA gene expression data to separate gene expression into genes targeted by the same miRNA predicted to target CCND1 from miRNAs that are not predicted to bind the CCND1 3'-UTR (Figure 4A). 7,396 genes share MREs with CCND1 ('targets"), while 6,230 do not ("controls"). When comparing gene expression normalized to log2FC, no differences were observed in the expression levels between these global gene groups in either HNSCC as a whole, or stratified by HPV-status (Figure 4B).

Target transcript degradation and downregulation is not the only mechanism employed by ceRNA. Target proteins can be downregulated through inhibiting translation, sequestering transcripts, or direct degradation of the transcript through exonuclease activity(31,61). These alternative translation inhibitory mechanisms may not lead to accelerated degradation of target transcripts and would not be discernible in a global context (Figure 4C). Therefore, it is possible that target proteins can be downregulated due to CCND1 amplification and overexpression through one of these non-degradative mechanisms, and/or that the CCND1 3'UTR effect is only manifest against a small number of targets that would not be discernible by global evaluation (Figure 4D). 


\section{CCND1 3'-UTR overexpression correlates with a subset of overexpressed proteins protein expression}

in HNSCC and other 11q13-amplified cancers

To evaluate alternative readouts of potential CCND1 3'-UTR ceRNA activity, TCGA RPPA data and correlated protein expression was correlated with increasing CCND1 3'-UTR expression (Figure 5A). Although the TCGA reverse phase protein lysate microarray (RPPA) dataset consists of only 160 different proteins, differences in protein expression were identified that correlated with CCND1 3'-UTR expression. In HPV-positive patients, eight proteins are negatively correlated significantly with increasing CCND1 3'-UTR expression. Of these, all but the transcripts of CDKN2A, C12ORF5, and CTNNA1 shared MREs with CCND1 (Figure 5D). We also observed four proteins (BID, RB1, PXN, and MACC1) that positively correlated significantly with increasing CCND1 3'-UTR expression and the transcripts of which share MREs with CCND1 (Figure 5B). In HPV-negative patients, nine proteins negatively correlated significantly with increasing CCND1 3'-UTR expression. Transcripts of PRKCA, MACC1, AKT1/2/3, and BAK1 share MREs with CCND1 (Figure 5C). In addition, 12 proteins positively correlate significantly with increasing CCND1 3'-UTR expression (Figure 5C). Of these, the transcripts of SERPINE1, TYMS, DVL3, ITGA2, BCL2L11, CCNB1, GATA3, and E2F1 share MREs with the CCND1 3'-UTR (Figure 5D).

Evaluating mRNA expression for these target proteins revealed altered transcript expression when stratified by CCND1 3'-UTR expression levels. Expression levels of E2F1, SERPINE1, and TYMS were significantly different when stratified by CCND1 3'-UTR expression, suggesting that CCND1 expression is connected to target protein expression through control of translation or transcript degradation. The expression levels of the remaining transcripts were not significantly different when stratified by CCND1 3'-UTR expression levels, but may be regulated by a ceRNA mechanism that does not utilize target transcript degradation.

In addition to HNSCC, several other cancer types have high levels of 11q13 amplification, including bladder(62), breast(63), esophageal squamous(64) and lung squamous cell carcinoma(65). To determine if the potential CCND1 3'-UTR ceRNA mechanism utilized in HNSCC is also present in these cancers, a parallel analysis was performed on TCGA-BLCA, TCGA-BRCA, TCGA-ESCA, and TCGA-LUSC. In TCGA-BLCA, while there were several significantly positively correlated proteins that also shared CCND1 MREs, no potential targets were shared with TCGA-HNSC. IGFBP2 and PARP1 are significantly negatively correlated and shared between TCGA-BLCA and TCGA-HNSC, but the transcripts do not share CCND1 MREs (Figure 6A). In TCGA-BRCA, 
PARP1, CCNB1, ANXA1, DVL3, EIF4EBP1, PRKCA, and MYC were significantly negatively correlated similarly to TCGA-HNSC, but only the transcripts of CCNB1, DVL3, and PRKCA share CCND1 MREs. Interestingly, shared positive correlations include ITGA2 and GATA3, which do share CCND1 MREs in the transcripts and are also seen in TCGA-HNSC (Figure 6B). In TCGA-ESCA, LCK was significantly negatively correlated and was shared with TCGA-HNSC. Significantly positively correlated genes that were shared with TCGA-HNSC include both SERPINE1 and ITGA2, which transcripts share CCND1 MREs (Figure 6C). In TCGA-LUSC, ITGA2 was the only significantly positively correlated protein, with the transcript sharing CCND1 MREs present in TCGAHNSC (Figure 6D). Other shared proteins were discordantly regulated across cancer types (Figure 6E).

When evaluating mRNA expression levels of proteins that are shared between CCND1 and TCGA-HNSC, the expression levels of ITGA2 and GATA3 in TCGA-BRCA and ITGA2 and SERPINE1 in TCGA-ESCA were all significantly different when stratified by CCND1 3'-UTR expression, suggesting that CCND1 3'-UTR expression levels has a role in regulating expression of these targets. In contrast, ITGA2 in TCGA-LUSC was not significantly different, suggesting a different regulatory mechanism of transcript and/or protein expression in this cancer type. 


\section{Discussion}

In this study, we propose a ceRNA network involving a novel function of CCND1 3'-UTR that may compete for tumor-suppressing miRNA, leading to dysregulation of target transcripts and alteration of the expression of target proteins with tumor-promoting functions. We identified several proteins that had altered expression when stratified by high and low expression of the 3'-UTR exon of CCND1, including TYMS, SERPINE1, CCNB1, DVL3, ITGA2, GATA3, and E2F1. The 3'-UTR of CCND1 has been confirmed to bind and regulated by multiple miRNA, including miRs-15, 16,17 , and 20 . In addition to miRNA, the transcript is also regulated post-transcriptionally by AU-rich element (ARE)-binding proteins (BPs) at seven putative sites that help regulate and stabilize the transcript(66). There is precedent for ceRNA networks in HNSCC as several ceRNA networks have already been described in HNSCC, but these do not include $C C N D 1(39,67,68)$. The concept of amplification leading to the overexpression of transcripts that can serve as ceRNA has also been previously described. These include the IncRNA PVT1 that is amplified in gastrointestinal cancer(69), the frequently amplified oncogene $C M Y C(70)$, and MYEOV in lung cancer, which arises from the same amplification event as CCND1(71). The CCND13-'UTR also has a function in oncogenic transformation of cells by acting as scaffolding for elF4E and subsequent assembly of ribonucleotides responsible for the modulation of cellular growth(60). The binding of any miRNA, ARE-BP, and other 3'-UTR-mediated functions, including any potential ceRNA-mediated function, requires the 3'-UTR to not undergo alternative cleavage and polyadenylation (APA) that is typically found in cancer cells(72). Recent studies have shown that this does not seem to occur for the CCND13'-UTR(73). These reports and our results allow for the potential for ceRNA-mediated function.

This study has identified several proteins that have altered expression when stratified by high and low expression of CCND1 3'-UTR, including TYMS, SERPINE1, CCNB1, DVL3, ITGA2, GATA3, and E2F1. Most of these proteins have described roles in tumor progression. Thymidylate synthase (encoded by TYMS) has a role in DNA synthesis through the generation of thymidine deoxynucleotides, which is an important need of quickly dividing cells found in tumors $(74,75)$. It has become a recent area of interest for targeting due to this role(76). Endothelial plasminogen activator inhibitor (encoded by SERPINE1) functions as the main inhibitor of tissue plasminogen activator and urokinase(77). However, a paradoxical role in cancer has been observed with overexpression of SERPINE1 being linked to tumor cell proliferation, migration, and invasion(77-80) and has 
been interesting as of late for targeting with small molecule inhibitors(81). G2/mitotic-specific cyclin B1 (encoded by $C C N B 1)$ is a cell cycle regulatory protein that complexes with CDK1 to regulate early cell cycle events(82) such as condensation of chromosomes(83). CCNB1 is similar to CCND1 in that it regulates cell cycle and aberrant expression can lead to increased cell cycle and increased tumor progression(84-88). Segment polarity protein disheveled homolog DVL-3 (encoded by DVL3) functions in the regulation of cell proliferation(89) and overexpression of DVL3 has been linked to increased cellular proliferation and migration(90,91). Integrin alpha2 (ITGA2) makes up part of the $\alpha 2 \beta 1$ integrin duplex and is involved in cell adhesion and cell-surface-mediated signaling and has been implicated in tumor progression(92-96). GATA binding protein 3 (encoded by GATA3) is a transcription factor that binds GATA DNA sequences and functions in the development of tissues(97) and T helper cells(98), secretion of interleukins, and also induces maturation of breast epithelial cells(99). Its role in cancer is controversial, as it has been shown to have tumor-promoting(100) and tumor-suppressing roles(101), likely due to different transcriptional targets. E2F1 (encoded by E2F1) is a transcription factor involved in the CDK-pRB pathway that is responsible for regulating the transcription of $S$ phase genes(102), including TYMS(103). Due to its function, overexpression of E2F1 has been implicated as a tumor-promoter in numerous cancer types(104-106) as well as a tumor-suppressor(107) depending on its transcriptional targets.

Importantly, all identified target genes share confirmed MREs with CCND1. Perhaps the most well-known is its ability to bind hsa-miR-16(108) which is shared with ITGA2(109). CCND1 has also been shown to bind hsa-miR19(110), shared with BCL2L11(111). Both BCL2L11 and E2F1 share hsa-miR-17(111,112), hsa-miR20(111,112), hsa-miR-106(113,114), and hsa-miR-302(115,116) with CCND1(117,118). SERPINE1 shares hsamiR-34(119) and hsa-miR-449(120) binding with CCND1(121,122). TYMS shares hsa-miR-203 binding(123) with CCND1(124). The miRNA hsa-miR-372 is shared between ITGA2(125), E2F1(126), and CCND1(127). Lastly, DVL3 shares binding sites for hsa-let-7(128) with CCND1(121). Other microRNA, namely hsa-miR-142, that have been confirmed to bind CCND1(129) have not yet been confirmed to bind CCNB1 or GATA3 are only predicted by TargetScan at this time.

This study also has implications in other cancer types with high 11q13 amplification rates. In esophageal squamous carcinoma, the expression levels of ITGA2 and SERPINE1 were altered when stratified by CCND1 3'-UTR expression. SERPINE1 has been demonstrated as a prognostic marker in esophageal carcinoma with 
overexpression being significantly higher in cancer patients and significantly correlates with smoking and patient age(130). Overexpression of SERPINE1 protein in esophageal squamous carcinoma has been linked to the promotion of invasion and migration through the activation of AKT and ERK signaling pathways(80). In lung cancer, ITGA2 is overexpressed in patients and is associated with clinical development of cancer(131), which is supportive of the findings in this study. In breast cancer, the expression levels of GATA3 and ITGA2 were significantly different when stratified by high and low CCND1 3'-UTR expression. Paradoxically, these proteins are reported to have both tumor-suppressive and tumor-promoting roles. Silencing of ITGA2 has been shown to promote cell migration(125), yet overexpression of ITGA2 can also lead to promoted cell proliferation(94). Loss of GATA3 is associated with invasive growth and poor prognosis(132), yet a stabilizing mutation found in GATA3 can lead to a growth advantage in tumors $(133,134)$ ). Overexpression of GATA3 through a ceRNA-mediated mechanism may mimic this stabilization effect. Stratification by CCND1 3'-UTR may elucidate the paradoxical roles of GATA3 and ITGA2 in breast cancer. Overall, this study underscores the importance of determining the expression of CCND1 mRNA, which may lead to better patient stratification for treatment or clinical trials. 


\section{Bibliography}

1. Vokes EE, Weichselbaum RR, Lippman SM, Hong WK. Head and neck cancer. N Engl J Med [Internet]. 1993 Jan 21;328(3):184-94. Available from: http://www.ncbi.nlm.nih.gov/pubmed/8417385

2. Ang KK, Harris J, Wheeler R, Weber R, Rosenthal DI, Nguyen-Tân PF, et al. Human papillomavirus and survival of patients with oropharyngeal cancer. N Engl J Med [Internet]. 2010 Jul 1;363(1):24-35. Available from:

http://www.ncbi.nlm.nih.gov/pubmed/20530316\%0Ahttp://www.pubmedcentral.nih.gov/articlerender.fcgi ?artid=PMC2943767

3. Chow LQM. Head and Neck Cancer. Longo DL, editor. N Engl J Med [Internet]. 2020 Jan 2 [cited 2020 May 4];382(1):60-72. Available from: http://www.nejm.org/doi/10.1056/NEJMra1715715

4. Argiris A, Karamouzis M V, Raben D, Ferris RL. Head and neck cancer. Lancet (London, England) [Internet]. 2008;371(9625):1695-709. Available from:

http://www.sciencedirect.com/science/article/pii/S014067360860728X

5. Hayes DN, Van Waes C, Seiwert TY. Genetic landscape of human papillomavirus-associated head and neck cancer and comparison to tobacco-related tumors. J Clin Oncol [Internet]. 2015 Oct 10 [cited 2020 Jun 19];33(29):3227-34. Available from: http://www.ncbi.nlm.nih.gov/pubmed/26351353

6. Leemans CR, Snijders PJF, Brakenhoff RH. The molecular landscape of head and neck cancer. Nat Rev Cancer. 2018;18(5):269-82.

7. Lawrence MS, Sougnez C, Lichtenstein L, Cibulskis K, Lander E, Gabriel SB, et al. Comprehensive genomic characterization of head and neck squamous cell carcinomas. Nature [Internet]. 2015;517(7536):576-82. Available from: http://www.nature.com/doifinder/10.1038/nature14129

8. Keck MK, Zuo Z, Khattri A, Stricker TP, Brown CD, Imanguli M, et al. Integrative Analysis of Head and Neck Cancer Identifies Two Biologically Distinct HPV and Three Non-HPV Subtypes. Clin Cancer Res [Internet]. 2015;21(4):870-81. Available from: http://clincancerres.aacrjournals.org/cgi/doi/10.1158/10780432.CCR-14-2481 
9. Gollin SM. Cytogenetic Alterations and their Molecular Genetic Correlates in Head and Neck Squamous Cell Carcinoma: A Next Generation Window to the Biology of Disease. Genes Chromosomes Cancer [Internet]. 2014 Dec [cited 2020 Jul 20];53(12):972-90. Available from:

https://pubmed.ncbi.nlm.nih.gov/25183546/

10. Gillison ML, Akagi K, Xiao W, Jiang B, Pickard RKL, Li J, et al. Human papillomavirus and the landscape of secondary genetic alterations in oral cancers. Genome Res [Internet]. 2019;29(1):1-17. Available from: http://www.ncbi.nlm.nih.gov/pubmed/30563911

11. Meredith SD, Levine PA, Burns JA, Gaffey MJ, Boyd JC, Weiss LM, et al. Chromosome 11q13 Amplification in Head and Neck Squamous Cell Carcinoma: Association With Poor Prognosis. Arch Otolaryngol - Head Neck Surg [Internet]. 1995 Jul 1;121(7):790-4. Available from: http://archotol.jamanetwork.com/article.aspx?articleid=622902

12. Schuuring $\mathrm{E}$. The involvement of the chromosome 11q13 region in human malignancies : cyclin D1 and EMS1 are two new candidate oncogenes- a review *. Gene [Internet]. 1995 [cited 2020 Jul 20];159(1):83-96. Available from: https://pubmed.ncbi.nlm.nih.gov/7607576/

13. Gollin SM. Chromosomal alterations in squamous cell carcinomas of the head and neck: Window to the biology of disease. Head Neck [Internet]. 2001 [cited 2020 Jul 20];23(3):238-53. Available from: https://pubmed.ncbi.nlm.nih.gov/11428456/

14. Lammie GA, Peters G. Chromosome 11 q13 abnormalities in human cancer [Internet]. Vol. 3, Cancer Cells. 1991 [cited 2020 Jul 20]. p. 413-20. Available from: https://europepmc.org/article/med/1760243

15. Matsushime H, Ewen ME, Strom DK, Kato JY, Hanks SK, Roussel MF, et al. Identification and properties of an atypical catalytic subunit (p34PSK-J3/cdk4) for mammalian D type G1 cyclins. Cell [Internet]. 1992 [cited 2020 Jul 20];71(2):323-34. Available from:

https://pubmed.ncbi.nlm.nih.gov/1423597/

16. Cheng H, Yang X, Si H, Saleh AD, Xiao W, Coupar J, et al. Genomic and Transcriptomic Characterization Links Cell Lines with Aggressive Head and Neck Cancers. Cell Rep [Internet]. 2018 
[cited 2020 Jul 20];25(5):1332-1345.e5. Available from: https://pubmed.ncbi.nlm.nih.gov/30380422/

17. Wang X, Pavelic ZP, Li YQ, Wang L, Gleich L, Radack K, et al. Amplification and overexpression of the cyclin D1 gene in head and neck squamous cell carcinoma. J Clin Pathol - Clin Mol Pathol [Internet]. 1995 [cited 2020 Jul 20];48(5). Available from: https://pubmed.ncbi.nlm.nih.gov/16696017/

18. van Kempen PMW, Noorlag R, Braunius WW, Moelans CB, Rifi W, Savola S, et al. Clinical relevance of copy number profiling in oral and oropharyngeal squamous cell carcinoma. Cancer Med [Internet]. 2015 [cited 2020 Jul 20];4(10):1525-35. Available from: https://pubmed.ncbi.nlm.nih.gov/26194878/

19. Michalides RJAM, Van Veelen NMJ, Kristel PMP, Hart AAM, Loftus BM, Hilgers FJM, et al. Overexpression of cyclin D1 indicates a poor prognosis in squamous cell carcinoma of the head and neck. Arch Otolaryngol - Head Neck Surg [Internet]. 1997 [cited 2020 Jul 20];123(5):497-502. Available from: https://pubmed.ncbi.nlm.nih.gov/9158396/

20. Wilkerson PM, Reis-Filho JS. The 11q13-q14 amplicon: clinicopathological correlations and potential drivers. Genes Chromosomes Cancer [Internet]. 2013 Apr [cited 2014 Jun 3];52(4):333-55. Available from: http://onlinelibrary.wiley.com/doi/10.1002/gcc.22037/full

21. Ramos-García P, Ruiz-Ávila I, Gil-Montoya JA, Ayén Á, González-Ruiz L, Navarro-Triviño FJ, et al. Relevance of chromosomal band $11 q 13$ in oral carcinogenesis: An update of current knowledge. Oral Oncol [Internet]. 2017 [cited 2020 Jul 21];72(2017):7-16. Available from: https://pubmed.ncbi.nlm.nih.gov/28797464/

22. Maruyama R, Suzuki H. Long noncoding rna involvement in cancer. BMB Rep [Internet]. 2012 [cited 2020 Jul 20];45(11):604-11. Available from: https://pubmed.ncbi.nlm.nih.gov/23186998/

23. Li X, Cao Y, Gong X, Li H. Long noncoding RNAs in head and neck cancer [Internet]. Vol. 8, Oncotarget. Oncotarget; 2017 [cited 2020 Jul 20]. p. 10726-40. Available from: https://pubmed.ncbi.nlm.nih.gov/27802187/

24. Beermann J, Piccoli MT, Viereck J, Thum T. Non-coding rnas in development and disease: Background, mechanisms, and therapeutic approaches. Physiol Rev [Internet]. 2016 [cited 2020 Jul 20];96(4):1297- 
325. Available from: https://pubmed.ncbi.nlm.nih.gov/27535639/

25. Piwecka M, Glažar P, Hernandez-Miranda LR, Memczak S, Wolf SA, Rybak-Wolf A, et al. Loss of a mammalian circular RNA locus causes miRNA deregulation and affects brain function. Science (80- ) [Internet]. 2017 [cited 2020 Jul 20];357(6357). Available from:

https://pubmed.ncbi.nlm.nih.gov/28798046/

26. Chiu HS, Martínez MR, Komissarova E V., Llobet-Navas D, Bansal M, Paull EO, et al. The number of titrated microRNA species dictates ceRNA regulation. Nucleic Acids Res [Internet]. 2018 [cited 2020 Jul 20];46(9):4354-69. Available from: https://pubmed.ncbi.nlm.nih.gov/29684207/

27. Kleaveland B, Shi CY, Stefano J, Bartel DP. A Network of Noncoding Regulatory RNAs Acts in the Mammalian Brain. Cell [Internet]. 2018 [cited 2020 Jul 20];174(2):350-362.e17. Available from: https://pubmed.ncbi.nlm.nih.gov/29887379/

28. Grüll MP, Massé E. Mimicry, deception and competition: The life of competing endogenous RNAs [Internet]. Vol. 10, Wiley Interdisciplinary Reviews: RNA. Wiley Interdiscip Rev RNA; 2019 [cited 2020 Jul 20]. Available from: https://pubmed.ncbi.nlm.nih.gov/30761752/

29. Anastasiadou E, Jacob LS, Slack FJ. Non-coding RNA networks in cancer [Internet]. Vol. 18, Nature Reviews Cancer. Nat Rev Cancer; 2017 [cited 2020 Jul 20]. p. 5-18. Available from: https://pubmed.ncbi.nlm.nih.gov/29170536/

30. Lim LP, Lau NC, Garrett-Engele P, Grimson A, Schelter JM, Castle J, et al. Microarray analysis shows that some microRNAs downregulate large numbers of-target mRNAs. Nature [Internet]. 2005 [cited 2020 Jul 20];433(7027):769-73. Available from: https://pubmed.ncbi.nlm.nih.gov/15685193/

31. Valencia-Sanchez MA, Liu J, Hannon GJ, Parker R. Control of translation and mRNA degradation by miRNAs and siRNAs. Genes Dev [Internet]. 2006 Mar 1 [cited 2014 Jul 14];20(5):515-24. Available from: http://www.ncbi.nlm.nih.gov/pubmed/16510870

32. Hu J, Li X, Guo X, Guo Q, Xiang C, Zhang Z, et al. The CCR2 3'UTR functions as a competing endogenous RNA to inhibit breast cancer metastasis. J Cell Sci [Internet]. 2017 [cited 2020 Jul 
20];130(19):3399-413. Available from: https://pubmed.ncbi.nlm.nih.gov/28818997/

33. Gao S, Cheng C, Chen H, Li M, Liu K, Wang G. IGF1 3'UTR functions as a ceRNA in promoting angiogenesis by sponging miR-29 family in osteosarcoma. J Mol Histol [Internet]. 2016 [cited 2020 Jul 20];47(2):135-43. Available from: https://pubmed.ncbi.nlm.nih.gov/26759259/

34. Zheng T, Chou J, Zhang F, Liu Y, Ni H, Li X, et al. CXCR4 3'UTR functions as a ceRNA in promoting metastasis, proliferation and survival of MCF-7 cells by regulating miR-146a activity. Eur J Cell Biol [Internet]. 2015 [cited 2020 Jul 20];94(10):458-69. Available from:

https://pubmed.ncbi.nlm.nih.gov/26095299/

35. Jeyapalan Z, Deng Z, Shatseva T, Fang L, He C, Yang BB. Expression of CD44 3'-untranslated region regulates endogenous microRNA functions in tumorigenesis and angiogenesis. Nucleic Acids Res [Internet]. 2011 [cited 2020 Jul 20];39(8):3026-41. Available from:

https://pubmed.ncbi.nlm.nih.gov/21149267/

36. Lee DY, Jeyapalan Z, Fang L, Yang J, Zhang Y, Yee AY, et al. Expression of versican 3'-untranslated region modulates endogenous microrna functions. PLoS One [Internet]. 2010 [cited 2020 Jul 20];5(10). Available from: https://pubmed.ncbi.nlm.nih.gov/21049042/

37. Tay Y, Rinn J, Pandolfi PP. The multilayered complexity of ceRNA crosstalk and competition. Nature [Internet]. 2014 Jan 15 [cited 2016 Aug 31];505(7483):344-52. Available from: http://www.nature.com/doifinder/10.1038/nature12986

38. Salmena L, Poliseno L, Tay Y, Kats L, Pandolfi PP. A ceRNA hypothesis: the Rosetta Stone of a hidden RNA language? Cell [Internet]. 2011 Aug 5 [cited 2014 Jul 9];146(3):353-8. Available from: http://www.cell.com/article/S0092867411008129/fulltext

39. Wang Z, Liu T, Li G, Cao Z. The exploration of new therapeutic targets for HPV-negative head and neck squamous cell cancer through the construction of a ceRNA network and immune microenvironment analysis. J Cell Biochem [Internet]. 2020 Jun 1 [cited 2020 Jul 16];121(5-6):3426-37. Available from: https://onlinelibrary.wiley.com/doi/abs/10.1002/jcb.29615 
40. Zhou RS, Zhang EX, Sun QF, Ye ZJ, Liu JW, Zhou DH, et al. Integrated analysis of IncRNA-miRNAmRNA ceRNA network in squamous cell carcinoma of tongue. BMC Cancer [Internet]. 2019 [cited 2020 Jul 20];19(1). Available from: https://pubmed.ncbi.nlm.nih.gov/31391008/

41. Ambros V. The functions of animal microRNAs. Nature [Internet]. 2004 [cited 2020 Jul 20];431(7006):350-5. Available from: http://dx.doi.org/10.1038/nature02871

42. Tu HF, Lin SC, Chang KW. MicroRNA aberrances in head and neck cancer: Pathogenetic and clinical significance [Internet]. Vol. 21, Current Opinion in Otolaryngology and Head and Neck Surgery. Curr Opin Otolaryngol Head Neck Surg; 2013 [cited 2020 Jul 20]. p. 104-11. Available from: https://pubmed.ncbi.nlm.nih.gov/23340306/

43. Sethi N, Wright A, Wood H, Rabbitts P. MicroRNAs and head and neck cancer: Reviewing the first decade of research [Internet]. Vol. 50, European Journal of Cancer. Eur J Cancer; 2014 [cited 2020 Jul 20]. p. 2619-35. Available from: https://pubmed.ncbi.nlm.nih.gov/25103455/

44. Koshizuka K, Hanazawa T, Fukumoto I, Kikkawa N, Okamoto Y, Seki N. The microRNA signatures: Aberrantly expressed microRNAs in head and neck squamous cell carcinoma [Internet]. Vol. 62, Journal of Human Genetics. J Hum Genet; 2017 [cited 2020 Jul 20]. p. 3-13. Available from: https://pubmed.ncbi.nlm.nih.gov/27557665/

45. Williams ME, Gaffey MJ, Weiss LM, Wilczynski SP, Schuuring E, Levine PA, et al. Chromosome 11Q13 Amplification in Head and Neck Squamous Cell Carcinoma. Arch Otolaryngol Neck Surg [Internet]. 1994 Nov 1 [cited 2020 Jun 19];119(11):1238-43. Available from: https://pubmed.ncbi.nlm.nih.gov/8217084/

46. Broad Institute TCGA Genome Data Analysis Center (2016): Firehose 2016_01_28 run. Broad Institute of MIT and Harvard. doi:10.7908/C11G0KM9.

47. Cerami E, Gao J, Dogrusoz U, Gross BE, Sumer SO, Aksoy BA, et al. The cBio Cancer Genomics Portal: An open platform for exploring multidimensional cancer genomics data. Cancer Discov. $2012 ; 2(5): 401-4$.

48. James Kent W, Sugnet CW, Furey TS, Roskin KM, Pringle TH, Zahler AM, et al. The human genome 
browser at UCSC. Genome Res. 2002;12(6):996-1006.

49. Thierry-Mieg D, Thierry-Mieg J. AceView: a comprehensive cDNA-supported gene and transcripts annotation. Genome Biol [Internet]. 2006 Aug 7 [cited 2020 Jul 20];7 Suppl 1(Suppl 1):S12. Available from: http://genomebiology.biomedcentral.com/articles/10.1186/gb-2006-7-s1-s12

50. Agarwal V, Bell GW, Nam JW, Bartel DP. Predicting effective microRNA target sites in mammalian mRNAs. Elife [Internet]. 2015 [cited 2020 Jul 20];4(AUGUST2015). Available from: https://pubmed.ncbi.nlm.nih.gov/26267216/

51. Wang D, Gu J, Wang T, Ding Z. OncomiRDB: A database for the experimentally verified oncogenic and tumor-suppressive microRNAs. Bioinformatics [Internet]. 2014 [cited 2020 Jul 20];30(15):2237-8. Available from: https://pubmed.ncbi.nlm.nih.gov/24651967/

52. Mermel CH, Schumacher SE, Hill B, Meyerson ML, Beroukhim R, Getz G. GISTIC2.0 facilitates sensitive and confident localization of the targets of focal somatic copy-number alteration in human cancers. Genome Biol [Internet]. 2011 [cited 2020 Jun 19];12(4):R41. Available from: http://www.ncbi.nlm.nih.gov/pubmed/21527027

53. Gatesman Ammer A, C. Kelley L, E. Hayes K, V. Evans J, Ann Lopez-Skinner L, H. Martin K, et al. Saracatinib Impairs Head and Neck Squamous Cell Carcinoma Invasion by Disrupting Invadopodia Function. J Cancer Sci Ther [Internet]. 2009 [cited 2020 Jul 20];01(02):052-61. Available from: https://pubmed.ncbi.nlm.nih.gov/20505783/

54. Rothschild BL, Shim AH, Ammer AG, Kelley LC, Irby KB, Head JA, et al. Cortactin overexpression regulates actin-related protein $2 / 3$ complex activity, motility, and invasion in carcinomas with chromosome 11q13 amplification. Cancer Res [Internet]. 2006 Aug 15 [cited 2020 Jul 13];66(16):801725. Available from: https://pubmed.ncbi.nlm.nih.gov/16912177/

55. Markwell SM, Ammer AG, Interval ET, Allen JL, Papenberg BW, Hames RA, et al. Cortactin phosphorylation by casein kinase 2 regulates actin-related protein 2/3 complex activity, invadopodia function, and tumor cell invasion. Mol Cancer Res [Internet]. 2019 [cited 2020 Jul 2];17(4):987-1001. 
Available from: https://www.ncbi.nlm.nih.gov/pmc/articles/PMC6445698/

56. White JS, Weissfeld JL, Ragin CCR, Rossie KM, Martin CL, Shuster M, et al. The influence of clinical and demographic risk factors on the establishment of head and neck squamous cell carcinoma cell lines. Oral Oncol [Internet]. 2007 [cited 2020 Jul 20];43(7):701-12. Available from: https://pubmed.ncbi.nlm.nih.gov/17112776/

57. Boukamp P, Petrussevska RT, Breitkreutz D, Hornung J, Markham A, Fusenig NE. Normal keratinization in a spontaneously immortalized aneuploid human keratinocyte cell line. J Cell Biol [Internet]. 1988 [cited 2020 Jul 20];106(3):761-71. Available from:

https://pubmed.ncbi.nlm.nih.gov/2450098/

58. Brenner JC, Graham MP, Kumar B, Saunders LM, Kupfer R, Lyons RH, et al. Genotyping of 73 UMSCC head and neck squamous cell carcinoma cell lines. Head Neck [Internet]. 2010 [cited 2020 Jul 20];32(4):417-26. Available from: https://pubmed.ncbi.nlm.nih.gov/19760794/

59. Coassin SR, Orjalo A V., Semaan SJ, Johansson HE. Simultaneous Detection of Nuclear and Cytoplasmic RNA Variants utilizing stellaris ${ }^{\circledR}$ RNA fluorescence in situ hybridization in adherent cells. Methods Mol Biol [Internet]. 2014 [cited 2020 Jul 20];1211:189-99. Available from: https://pubmed.ncbi.nlm.nih.gov/25218386/

60. Culjkovic B, Topisirovic I, Skrabanek L, Ruiz-Gutierrez M, Borden KLBB. elF4E promotes nuclear export of cyclin D1 mRNAs via an element in the 3'UTR. J Cell Biol [Internet]. 2005 Apr 25 [cited 2016 Mar 16];169(2):245-56. Available from: https://www.ncbi.nlm.nih.gov/pmc/articles/PMC2171863/

61. Jo MH, Shin S, Jung SR, Kim E, Song JJ, Hohng S. Human Argonaute 2 Has Diverse Reaction Pathways on Target RNAs. Mol Cell [Internet]. 2015 [cited 2020 Jul 7];59(1):117-24. Available from: https://pubmed.ncbi.nlm.nih.gov/26140367/

62. Zaharieva BM, Simon R, Diener PA, Ackermann D, Maurer R, Alund G, et al. High-throughput tissue microarray analysis of 11ql3 gene amplification (CCND1, FGF3, FGF4, EMS1) in urinary bladder cancer. J Pathol [Internet]. 2003 [cited 2020 Jul 20];201(4):603-8. Available from: 
https://pubmed.ncbi.nlm.nih.gov/14648664/

63. Lin CY, Vennam S, Purington N, Lin E, Varma S, Han S, et al. Genomic landscape of ductal carcinoma in situ and association with progression. Breast Cancer Res Treat [Internet]. 2019 [cited 2020 Jul 20];178(2):307-16. Available from: https://pubmed.ncbi.nlm.nih.gov/31420779/

64. Salem ME, Puccini A, Xiu J, Raghavan D, Lenz H, Korn WM, et al. Comparative Molecular Analyses of Esophageal Squamous Cell Carcinoma, Esophageal Adenocarcinoma, and Gastric Adenocarcinoma. Oncologist [Internet]. 2018 [cited 2020 Jul 20];23(11):1319-27. Available from: https://pubmed.ncbi.nlm.nih.gov/29866946/

65. Luk C, Tsao MS, Bayani J, Shepherd F, Squire JA. Molecular cytogenetic analysis of non-small cell lung carcinoma by spectral karyotyping and comparative genomic hybridization. Cancer Genet Cytogenet [Internet]. 2001 [cited 2020 Jul 20];125(2):87-99. Available from:

https://pubmed.ncbi.nlm.nih.gov/11369051/

66. Deshpande AAJA, Pastore A, Deshpande AAJA, Zimmermann Y, Hutter G, Weinkauf M, et al. 3'UTR mediated regulation of the cyclin D1 proto-oncogene. Cell Cycle [Internet]. 2009 [cited 2020 Jul 20];8(21):3584-92. Available from: https://pubmed.ncbi.nlm.nih.gov/19823025/

67. Fang XN, Yin M, Li H, Liang C, Xu C, Yang GW, et al. Comprehensive analysis of competitive endogenous RNAs network associated with head and neck squamous cell carcinoma. Sci Rep [Internet]. 2018 Jul 12 [cited 2020 Jul 16];8(1):10544. Available from:

https://www.ncbi.nlm.nih.gov/pmc/articles/PMC6043529/

68. Pan Y, Liu G, Wang D, Li Y. Analysis of IncRNA-Mediated ceRNA Crosstalk and Identification of Prognostic Signature in Head and Neck Squamous Cell Carcinoma. Front Pharmacol [Internet]. 2019 [cited 2020 Jul 16];10:150. Available from: https:/www.ncbi.nlm.nih.gov/pmc/articles/PMC6409312/

69. Martínez-Barriocanal Á, Arango D, Dopeso H. PVT1 Long Non-coding RNA in Gastrointestinal Cancer [Internet]. Vol. 10, Frontiers in Oncology. Front Oncol; 2020 [cited 2020 Jul 20]. Available from: https://pubmed.ncbi.nlm.nih.gov/32083000/ 
70. Chen PC, Yu CC, Huang WY, Huang WH, Chuang YM, Lin RI, et al. c-Myc acts as a competing endogenous RNA to sponge miR-34a, in the upregulation of CD44, in urothelial carcinoma. Cancers (Basel) [Internet]. 2019 [cited 2020 Jul 20];11(10). Available from:

https://pubmed.ncbi.nlm.nih.gov/31569404/

71. Fang L, Wu S, Zhu X, Cai J, Wu J, He Z, et al. MYEOV functions as an amplified competing endogenous RNA in promoting metastasis by activating TGF- $\beta$ pathway in NSCLC. Oncogene [Internet]. 2019 Feb 4 [cited 2020 Jul 7];38(6):896-912. Available from: http://www.nature.com/articles/s41388018-0484-9

72. Li L, Wang D, Xue M, Mi X, Liang Y, Wang P. 3'UTR shortening identifies high-risk cancers with targeted dysregulation of the ceRNA network. Sci Rep [Internet]. 2014 Jan 23 [cited 2016 Mar 21];4(1):5406. Available from: https://pubmed.ncbi.nlm.nih.gov/24953077/

73. Park HJ, Ji P, Kim S, Xia Z, Rodriguez B, Li L, et al. 3' UTR shortening represses tumor-suppressor genes in trans by disrupting ceRNA crosstalk. Nat Genet [Internet]. 2018 Jun 21 [cited 2020 Jul 16];50(6):783-9. Available from: http://www.nature.com/articles/s41588-018-0118-8

74. Peters GJ, Backus HHJ, Freemantle S, Van Triest B, Codacci-Pisanelli G, Van der Wilt CL, et al. Induction of thymidylate synthase as a 5-fluorouracil resistance mechanism [Internet]. Vol. 1587, Biochimica et Biophysica Acta - Molecular Basis of Disease. Biochim Biophys Acta; 2002 [cited 2020 Jul 13]. p. 194-205. Available from: http://www.ncbi.nlm.nih.gov/pubmed/12084461

75. Hardy LW, Finer-Moore JS, Montfort WR, Jones MO, Santi D V, Stroud RM. Atomic structure of thymidylate synthase: Target for rational drug design. Science (80- ) [Internet]. 1987 Jan 23 [cited 2020 Jul 13];235(4787):448-55. Available from: http://www.ncbi.nlm.nih.gov/pubmed/3099389

76. Alzhrani ZMM, Alam MM, Neamatallah T, Nazreen S. Design, synthesis and in vitro antiproliferative activity of new thiazolidinedione-1,3,4-oxadiazole hybrids as thymidylate synthase inhibitors. J Enzyme Inhib Med Chem [Internet]. 2020 Dec [cited 2020 Jul 13];35(1):1116-23. Available from: http://www.ncbi.nlm.nih.gov/pubmed/32354237 
77. Kubala MH, DeClerck YA. The plasminogen activator inhibitor-1 paradox in cancer: a mechanistic understanding [Internet]. Vol. 38, Cancer and Metastasis Reviews. Cancer Metastasis Rev; 2019 [cited 2020 Jul 13]. p. 483-92. Available from: http://www.ncbi.nlm.nih.gov/pubmed/31734763

78. Yang JD, Ma L, Zhu Z. SERPINE1 as a cancer-promoting gene in gastric adenocarcinoma: facilitates tumour cell proliferation, migration, and invasion by regulating EMT. J Chemother [Internet]. 2019 [cited 2020 Jul 13];31(7-8):408-18. Available from: http://www.ncbi.nlm.nih.gov/pubmed/31724495

79. Vachher M, Arora K, Burman A, Kumar B. NAMPT, GRN, and SERPINE1 signature as predictor of disease progression and survival in gliomas. J Cell Biochem [Internet]. 2020 Apr 11 [cited 2020 Jul 13];121(4):3010-23. Available from: https://pubmed.ncbi.nlm.nih.gov/31710121/

80. Wang D, Yang LY, Liu Z, Yu J, Zhang MJ, Zhang Y, et al. PAl-1 overexpression promotes invasion and migration of esophageal squamous carcinoma cells. Yi chuan = Hered [Internet]. 2020 Mar 20 [cited 2020 Jul 13];42(3):287-95. Available from: https://pubmed.ncbi.nlm.nih.gov/32217514/

81. Elokdah H, Abou-Gharbia M, Hennan JK, McFarlane G, Mugford CP, Krishnamurthy G, et al. Tiplaxtinin, a novel, orally efficacious inhibitor of plasminogen activator inhibitor-1: Design, synthesis, and preclinical characterization. J Med Chem [Internet]. 2004 Jul 1 [cited 2020 Jul 13];47(14):3491-4. Available from: http://www.ncbi.nlm.nih.gov/pubmed/15214776

82. Fang F, Newport JW. Evidence that the G1-S and G2-M transitions are controlled by different cdc2 proteins in higher eukaryotes. Cell [Internet]. 1991 Aug 23 [cited 2020 Jul 13];66(4):731-42. Available from: http://www.ncbi.nlm.nih.gov/pubmed/1652371

83. Song X, Du R, Gui H, Zhou M, Zhong W, Mao C, et al. Identification of potential hub genes related to the progression and prognosis of hepatocellular carcinoma through integrated bioinformatics analysis.

Oncol Rep [Internet]. 2020 Jan [cited 2020 Jul 13];43(1):133-46. Available from:

http://www.ncbi.nlm.nih.gov/pubmed/31746405

84. Yuan J, Krämer A, Matthess Y, Yan R, Spänkuch B, Gätje R, et al. Stable gene silencing of cyclin B1 in tumor cells increases susceptibility to taxol and leads to growth arrest in vivo. Oncogene [Internet]. 2006 
Mar 16 [cited 2020 Jul 13];25(12):1753-62. Available from:

http://www.ncbi.nlm.nih.gov/pubmed/16278675

85. Kushner J, Bradley G, Young B, Jordan RCK. Aberrant expression of cyclin A and cyclin B1 proteins in oral carcinoma. J Oral Pathol Med [Internet]. 2007 Feb [cited 2020 Jul 13];28(2):77-81. Available from: http://www.ncbi.nlm.nih.gov/pubmed/9950254

86. Kawamoto H, Koizumi H, Uchikoshi T. Expression of the G2-M checkpoint regulators cyclin B1 and cdc2 in nonmalignant and malignant human breast lesions: Immunocytochemical and quantitative image analyses. Am J Pathol [Internet]. 1997 Jan [cited 2020 Jul 13];150(1):15-23. Available from: http://www.ncbi.nlm.nih.gov/pubmed/9006317

87. Wang A, Yoshimi N, Ino N, Tanaka T, Mori H. Overexpression of cyclin B1 in human colorectal cancers. J Cancer Res Clin Oncol [Internet]. 1997 [cited 2020 Jul 13];123(2):124-7. Available from: http://www.ncbi.nlm.nih.gov/pubmed/9030252

88. Mashal RD, Lester S, Corless C, Richie JP, Chandra R, Propert KJ, et al. Expression of cell cycleregulated proteins in prostate cancer. Cancer Res [Internet]. 1996 Sep 15 [cited 2020 Jul 13];56(18):4159-63. Available from: http://www.ncbi.nlm.nih.gov/pubmed/8797586

89. Pizzuti A, Amati F, Calabrese G, Mari A, Colosimo A, Silani V, et al. cDNA characterization and chromosomal mapping of two human homologues of the Drosophila dishevelled polarity gene. Hum Mol Genet [Internet]. 1996 Jul [cited 2020 Jul 13];5(7):953-8. Available from: http://www.ncbi.nlm.nih.gov/pubmed/8817329

90. Li K, Zhang J, Tian Y, He Y, Xu X, Pan W, et al. The Wnt/ 3 -catenin/VASP positive feedback loop drives cell proliferation and migration in breast cancer. Oncogene [Internet]. 2020 Mar [cited 2020 Jul 13];39(11):2258-74. Available from: http://www.ncbi.nlm.nih.gov/pubmed/31831834

91. Karin-Kujundzic V, Kardum V, Sola IM, Paic F, Skrtic A, Skenderi F, et al. Dishevelled family proteins in serous ovarian carcinomas: a clinicopathologic and molecular study. APMIS [Internet]. 2020 Mar [cited 2020 Jul 13];128(3):201-10. Available from: http://www.ncbi.nlm.nih.gov/pubmed/31755579 
92. Langsenlehner U, Renner W, Yazdani-Biuki B, Eder T, Wascher TC, Paulweber B, et al. Integrin alpha-2 and beta-3 gene polymorphisms and breast cancer risk. Breast Cancer Res Treat [Internet]. 2006 May [cited 2020 Jul 13];97(1):67-72. Available from: http://www.ncbi.nlm.nih.gov/pubmed/16317580

93. Gerger A, Hofmann G, Langsenlehner U, Renner W, Weitzer W, Wehrschütz M, et al. Integrin alpha-2 and beta-3 gene polymorphisms and colorectal cancer risk. Int J Colorectal Dis [Internet]. 2009 Feb [cited 2020 Jul 13];24(2):159-63. Available from: http://www.ncbi.nlm.nih.gov/pubmed/18836731

94. Ren D, Zhao J, Sun Y, Li D, Meng Z, Wang B, et al. Overexpressed ITGA2 promotes malignant tumor aggression by up-regulating PD-L1 expression through the activation of the STAT3 signaling pathway. $J$ Exp Clin Cancer Res [Internet]. 2019 Dec 9 [cited 2020 Jul 13];38(1):485. Available from: http://www.ncbi.nlm.nih.gov/pubmed/31818309

95. Ma L, Sun Y, Li D, Li H, Jin X, Ren D. Overexpressed ITGA2 contributes to paclitaxel resistance by ovarian cancer cells through the activation of the AKT/FoxO1 pathway. Aging (Albany NY) [Internet]. 2020 [cited 2020 Jul 13];12(6):5336-51. Available from: http://www.ncbi.nlm.nih.gov/pubmed/32202508

96. Wang L, Gao Y, Zhao X, Guo C, Wang X, Yang Y, et al. HOXD3 was negatively regulated by YY1 recruiting HDAC1 to suppress progression of hepatocellular carcinoma cells via ITGA2 pathway. Cell Prolif [Internet]. 2020 Jun 17 [cited 2020 Jul 13];e12835. Available from:

http://www.ncbi.nlm.nih.gov/pubmed/32557953

97. IC H, SY P. GATA-3 - not just for Th2 cells anymore. Cell Mol Immunol [Internet]. 2007 Feb 1 [cited 2020 Jul 21];4(1):15-29. Available from: https://europepmc.org/article/med/17349208

98. Yagi R, Zhu J, Paul WE. An updated view on transcription factor GATA3-mediated regulation of Th1 and Th2 cell differentiation [Internet]. Vol. 23, International Immunology. Int Immunol; 2011 [cited 2020 Jul 15]. p. 415-20. Available from: http://www.ncbi.nlm.nih.gov/pubmed/21632975

99. Kouros-Mehr H, Slorach EM, Sternlicht MD, Werb Z. GATA-3 Maintains the Differentiation of the Luminal Cell Fate in the Mammary Gland. Cell [Internet]. 2006 Dec 1 [cited 2020 Jul 15];127(5):104155. Available from: http://www.ncbi.nlm.nih.gov/pubmed/17129787 
100. Shan L, Li X, Liu L, Ding X, Wang Q, Zheng Y, et al. GATA3 cooperates with PARP1 to regulate CCND1 transcription through modulating histone H1 incorporation. Oncogene [Internet]. 2014 Jun 12 [cited 2020 Jul 15];33(24):3205-16. Available from: http://www.ncbi.nlm.nih.gov/pubmed/23851505

101. Asselin-Labat M-L, Sutherland KD, Vaillant F, Gyorki DE, Wu D, Holroyd S, et al. Gata-3 Negatively Regulates the Tumor-Initiating Capacity of Mammary Luminal Progenitor Cells and Targets the Putative Tumor Suppressor Caspase-14. Mol Cell Biol [Internet]. 2011 Nov [cited 2020 Jul 16];31(22):4609-22. Available from: http://www.ncbi.nlm.nih.gov/pubmed/21930782

102. Neuman E, Sellers WR, McNeil JA, Lawrence JB, Kaelin WG. Structure and partial genomic sequence of the human E2F1 gene. Gene [Internet]. 1996 Sep 16 [cited 2020 Jul 16];173(2):163-9. Available from: http://www.ncbi.nlm.nih.gov/pubmed/8964493

103. DeGregori J, Kowalik T, Nevins JR. Cellular targets for activation by the E2F1 transcription factor include DNA synthesis- and G1/S-regulatory genes. Mol Cell Biol [Internet]. 1995 [cited 2020 Jul 3];15(8):4215-24. Available from: https://www.ncbi.nlm.nih.gov/pmc/articles/PMC230660/

104. Yin Y, Tan Y, Yao Y, Lu N, Zhang F. SNHG12/miR-326/E2F1 feedback loop facilitates the progression of oral squamous cell carcinoma. Oral Dis [Internet]. 2020 Jun 7 [cited 2020 Jul 16]; Available from: http://www.ncbi.nlm.nih.gov/pubmed/32506729

105. Suzuki T, Yasui W, Yokozaki H, Naka K, Ishikawa T, Tahara E. Expression of the E2F family in human gastrointestinal carcinomas. Int J Cancer [Internet]. 1999 May 17 [cited 2020 Jul 16];81(4):535-8. Available from: http://www.ncbi.nlm.nih.gov/pubmed/10225440

106. Johnson DG, Cress WD, Jakoi L, Nevins JR. Oncogenic capacity of the E2F1 gene. Proc Natl Acad Sci U S A [Internet]. 1994 Dec 20 [cited 2020 Jul 16];91(26):12823-7. Available from: http://www.ncbi.nlm.nih.gov/pubmed/7809128

107. Wang $\mathrm{Y}$, Wang G, Ma Y, Teng J, Wang $\mathrm{Y}$, Cui $\mathrm{Y}$, et al. FAT1, a direct transcriptional target of E2F1, suppresses cell proliferation, migration and invasion in esophageal squamous cell carcinoma. Chinese $\mathrm{J}$ Cancer Res [Internet]. 2019 Aug [cited 2020 Jul 16];31(4):609-19. Available from: 
http://www.ncbi.nlm.nih.gov/pubmed/31564804

108. Cai CK, Zhao GY, Tian LY, Liu L, Yan K, Ma YL, et al. miR-15a and miR-16-1 downregulate CCND1 and induce apoptosis and cell cycle arrest in osteosarcoma. Oncol Rep [Internet]. 2012 [cited 2020 Jul 20];28(5):1764-70. Available from: https://pubmed.ncbi.nlm.nih.gov/22922827/

109. Xu Y, Shen L, Li F, Yang J, Wan X, Ouyang M. microRNA-16-5p-containing exosomes derived from bone marrow-derived mesenchymal stem cells inhibit proliferation, migration, and invasion, while promoting apoptosis of colorectal cancer cells by downregulating ITGA2. J Cell Physiol [Internet]. 2019 [cited 2020 Jul 20];234(11):21380-94. Available from: https://pubmed.ncbi.nlm.nih.gov/31102273/

110. Qin X, Wang X, Wang Y, Tang Z, Cui Q, Xi J, et al. MicroRNA-19a mediates the suppressive effect of laminar flow on cyclin D1 expression in human umbilical vein endothelial cells. Proc Natl Acad Sci U S A [Internet]. 2010 [cited 2020 Jul 21];107(7):3240. Available from:

https://www.ncbi.nlm.nih.gov/pmc/articles/PMC2840357/

111. Scherr M, Elder A, Battmer K, Barzan D, Bomken S, Ricke-Hoch M, et al. Differential expression of miR17 92 identifies BCL2 as a therapeutic target in BCR-ABL-positive B-lineage acute lymphoblastic leukemia. Leukemia [Internet]. 2014 [cited 2020 Jul 20];28(3):554-65. Available from: https://www.ncbi.nlm.nih.gov/pmc/articles/PMC3948162/

112. Pickering MT, Stadler BM, Kowalik TF. miR-17 and miR-20a temper an E2F1-induced G1 checkpoint to regulate cell cycle progression. Oncogene [Internet]. 2009 [cited 2020 Jul 20];28(1):140-5. Available from: https://www.ncbi.nIm.nih.gov/pmc/articles/PMC2768269/

113. Tang W, Li J, Liu H, Zhou F, Liu M. MiR-106a promotes tumor growth, migration, and invasion by targeting BCL2L11 in human endometrial adenocarcinoma. Am J Transl Res [Internet]. 2017 [cited 2020 Jul 20];9(11):4984-93. Available from: https://www.ncbi.nlm.nih.gov/pmc/articles/PMC5714782/

114. Petrocca F, Visone R, Onelli MR, Shah MH, Nicoloso MS, de Martino I, et al. E2F1-Regulated MicroRNAs Impair TGF $\beta$-Dependent Cell-Cycle Arrest and Apoptosis in Gastric Cancer. Cancer Cell [Internet]. 2008 [cited 2020 Jul 20];13(3):272-86. Available from: 
https://pubmed.ncbi.nlm.nih.gov/18328430/

115. Tan J, Hu L, Yang X, Zhang X, Wei C, Lu Q, et al. miRNA expression profiling uncovers a role of miR$302 b-3 p$ in regulating skin fibroblasts senescence. J Cell Biochem [Internet]. 2020 [cited 2020 Jul 20];121(1):70-80. Available from: https://pubmed.ncbi.nlm.nih.gov/31074095/

116. Cataldo A, Cheung DG, Balsari A, Tagliabue E, Coppola V, lorio M V., et al. miR-302b enhances breast cancer cell sensitivity to cisplatin by regulating E2F1 and the cellular DNA damage response. Oncotarget [Internet]. 2016 [cited 2020 Jul 20];7(1):786-97. Available from: https://www.ncbi.nlm.nih.gov/pmc/articles/PMC4808033/

117. Trompeter HI, Abbad H, Iwaniuk KM, Hafner M, Renwick N, Tuschl T, et al. MicroRNAs MiR-17, MiR20a, and MiR-106b Act in concert to modulate E2F activity on cell cycle arrest during neuronal lineage differentiation of USSC. PLoS One [Internet]. 2011 [cited 2020 Jul 20];6(1). Available from: https://pubmed.ncbi.nlm.nih.gov/21283765/

118. Li HL, Wei JF, Fan LY, Wang SH, Zhu L, Li TP, et al. MiR-302 regulates pluripotency, teratoma formation and differentiation in stem cells via an AKT1/OCT4-dependent manner. Cell Death Dis [Internet]. 2016 [cited 2020 Jul 20];7(1). Available from: https://pubmed.ncbi.nlm.nih.gov/26821070/

119. Akula SM, Ruvolo PP, McCubrey JA. TP53/miR-34a-associated signaling targets SERPINE1 expression in human pancreatic cancer. Aging (Albany NY) [Internet]. 2020 [cited 2020 Jul 20];12(3):2777-97. Available from: https:/www.ncbi.nlm.nih.gov/pmc/articles/PMC7041729/

120. Muth M, Theophile K, Hussein K, Jacobi C, Kreipe H, Bock O. Hypoxia-induced down-regulation of microRNA-449a/b impairs control over targeted SERPINE1 (PAI-1) mRNA - a mechanism involved in SERPINE1 (PAI-1) overexpression. J Transl Med [Internet]. 2010 [cited 2020 Jul 20];8. Available from: https://pubmed.ncbi.nlm.nih.gov/20356416/

121. Hydbring P, Wang Y, Fassl A, Li X, Matia V, Otto T, et al. Cell-Cycle-Targeting MicroRNAs as Therapeutic Tools against Refractory Cancers. Cancer Cell [Internet]. 2017 [cited 2020 Jul 20];31(4):576-590.e8. Available from: https://pubmed.ncbi.nlm.nih.gov/28399412/ 
122. Fang Y, Gu X, Li Z, Xiang J, Chen Z. miR-449b inhibits the proliferation of SW1116 colon cancer stem cells through downregulation of CCND1 and E2F3 expression. Oncol Rep [Internet]. 2013 [cited 2020 Jul 20];30(1):399-406. Available from: https://pubmed.ncbi.nlm.nih.gov/23674142/

123. Li T, Gao F, Zhang XP. MiR-203 enhances chemosensitivity to 5 -fluorouracil by targeting thymidylate synthase in colorectal cancer. Oncol Rep [Internet]. 2015 [cited 2020 Jul 20];33(2):607-14. Available from: https://pubmed.ncbi.nIm.nih.gov/25482885/

124. Lohcharoenkal W, Harada M, Lovén J, Meisgen F, Landén NX, Zhang L, et al. MicroRNA-203 Inversely Correlates with Differentiation Grade, Targets c-MYC, and Functions as a Tumor Suppressor in cSCC. J Invest Dermatol [Internet]. 2016 [cited 2020 Jul 20];136(12):2485-94. Available from: https://pubmed.ncbi.nlm.nih.gov/27452220/

125. Ding W, Fan XL, Xu X, Huang JZ, Xu SH, Geng Q, et al. Epigenetic Silencing of ITGA2 by MiR-373 promotes cell migration in breast cancer. PLoS One [Internet]. 2015 [cited 2020 Jul 20];10(8). Available from: https://www.ncbi.nIm.nih.gov/pmc/articles/PMC4530956/

126. Zhao YX, Liu HC, Ying WY, Wang CY, Yu YJ, Sun WJ, et al. MicroRNA-372 inhibits proliferation and induces apoptosis in human breast cancer cells by directly targeting E2F1. Mol Med Rep [Internet]. 2017 [cited 2020 Jul 20];16(6):8069-75. Available from:

https://www.ncbi.nlm.nih.gov/pmc/articles/PMC5779890/

127. Verdelli C, Forno I, Morotti A, Creo P, Guarnieri V, Scillitani A, et al. The aberrantly expressed miR-372 partly impairs sensitivity to apoptosis in parathyroid tumor cells. Endocr Relat Cancer [Internet]. 2018 [cited 2020 Jul 20];25(7):761-71. Available from: https://pubmed.ncbi.nlm.nih.gov/29724878/

128. Xie Y, Zhang H, Guo XJ, Feng YC, He RZ, Li X, et al. Let-7c inhibits cholangiocarcinoma growth but promotes tumor cell invasion and growth at extrahepatic sites article. Cell Death Dis [Internet]. 2018 [cited 2020 Jul 20];9(2). Available from: https://pubmed.ncbi.nlm.nih.gov/29445149/

129. Su Y, Wang J, Ma Z, Gong W, Yu L. MiR-142 Suppresses Endometrial Cancer Proliferation in Vitro and in Vivo by Targeting Cyclin D1. DNA Cell Biol [Internet]. 2019 [cited 2020 Jul 20];38(2):144-50. 
Available from: https://pubmed.ncbi.nlm.nih.gov/30585737/

130. Klimczak-Bitner AA, Kordek R, Bitner J, Musiał J, Szemraj J. Expression of MMP9, SERPINE1 and miR 134 as prognostic factors in esophageal cancer. Oncol Lett [Internet]. 2016 [cited 2020 Jul 20];12(5):4133-8. Available from: https://www.ncbi.nlm.nih.gov/pmc/articles/PMC5104243/

131. Huang CW, Hsieh WC, Hsu ST, Lin YW, Chung YH, Chang WC, et al. The use of PET imaging for prognostic integrin $\alpha 2 \beta 1$ phenotyping to detect non-small cell lung cancer and monitor drug resistance responses. Theranostics [Internet]. 2017 [cited 2020 Jul 20];7(16):4013-28. Available from: https://pubmed.ncbi.nlm.nih.gov/29109795/

132. Mehra R, Varambally S, Ding L, Shen R, Sabel MS, Ghosh D, et al. Identification of GATA3 as a breast cancer prognostic marker by global gene expression meta-analysis. Cancer Res [Internet]. 2005 [cited 2020 Jul 20];65(24):11259-64. Available from: https://pubmed.ncbi.nlm.nih.gov/16357129/

133. Adomas AB, Grimm SA, Malone C, Takaku M, Sims JK, Wade PA. Breast tumor specific mutation in GATA3 affects physiological mechanisms regulating transcription factor turnover. BMC Cancer [Internet]. 2014 [cited 2020 Jul 20];14(1). Available from: https://pubmed.ncbi.nlm.nih.gov/24758297/

134. Takaku M, Grimm SA, Wade PA. GATA3 in breast cancer: Tumor suppressor or oncogene? [Internet]. Vol. 16, Gene Expression. NIH Public Access; 2015 [cited 2020 Jul 20]. p. 163-8. Available from: https://www.ncbi.nlm.nih.gov/pmc/articles/PMC4758516/ 
A. Chr11 Amplification Frequency

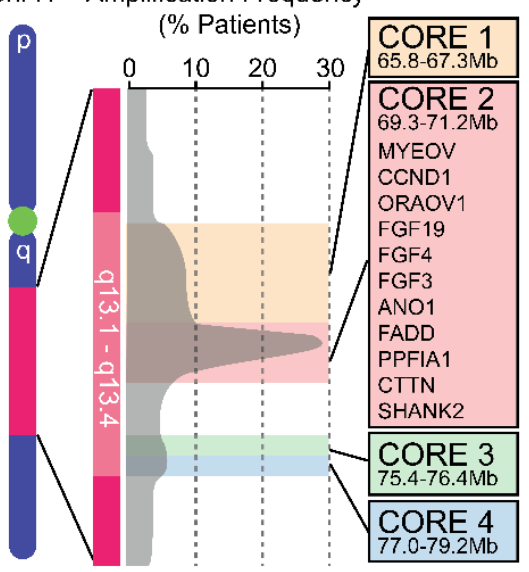

B.

\begin{tabular}{|c|c|c|c|c|}
\hline $\begin{array}{l}\text { CORE } 2 \\
\text { Gene Symbol }\end{array}$ & ORF Length $(b p)$ & 3'UTR Length (bp) & $\begin{array}{r}\text { Amplification in } \\
\text { TCGA-HNSC (\%) }\end{array}$ & $\begin{array}{r}\text { Amplification and } \\
\text { Overexpression of } \\
\text { 3'UTR Exon in } \\
\text { TCGA-HNSC (\%) }\end{array}$ \\
\hline MYEOV & 939 & 895 & 24.6 & 3.846 \\
\hline CCND1 & 885 & 3,192 & 26.9 & 15.387 \\
\hline ORAOV1 & 411 & 1,956 & 26.9 & 23.077 \\
\hline FGF19 & 648 & 1,243 & 26.9 & 0.050 \\
\hline FGF4 & 618 & 280 & 26.9 & 0.007 \\
\hline FGF3 & 717 & 677 & 26.9 & 0.023 \\
\hline ANO1 & 2,958 & 1,544 & 27.7 & 14.615 \\
\hline FADD & 624 & 929 & 27.7 & 27.307 \\
\hline PPFIA1 & 3,606 & 1,404 & 28.1 & 27.308 \\
\hline CTTN & 1,650 & 1,421 & 27.3 & 24.615 \\
\hline SHANK2 & 4,410 & 5,013 & 26.9 & 3.077 \\
\hline
\end{tabular}

C.

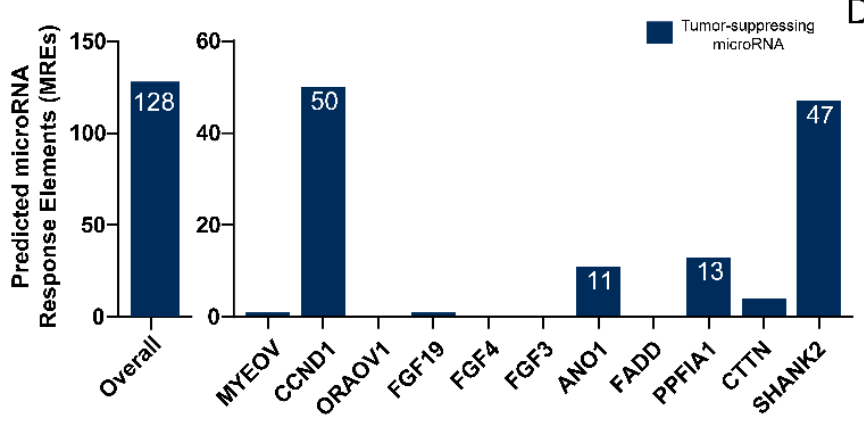

D. Selection of Predicted Tumor-suppressive CCND1 MREs miR-1-3p miR-16-5p miR-19-3p miR-34-5p miR-98-5p miR-142-3p miR-193-3p miR-302-3p miR-446-5p miR-519-3p
miR-15-5p miR-17-5p miR-20-3p miR-93-5p miR-106-5p $\mathrm{miR}-142-5 p$ miR-195-5p miR-442-5p miR-497-3p Let-7-5p

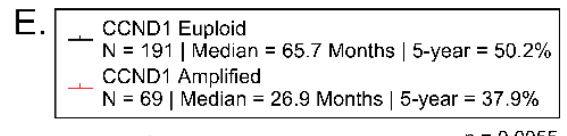

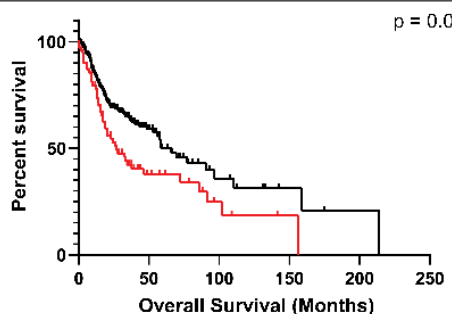

Figure 1. The 11q13 amplicon contains genes with long 3'-UTRs that have tumor-suppressing MREs. A.

The 11q13 amplified region displaying amplification frequency (grey) with defined cores of amplification (orange, red, green, blue). B. Genes within the core 2 region of the $11 q 13$ amplicon with ORF length, 3'-UTR length, amplification frequency, and 3'-UTR exon overexpression frequency. C. Predicted tumor-suppressing MREs of genes within the 11 q13 amplicon. D. Selection of tumor-suppressive miRNAs predicted to bind CCND1 3'-UTR. E. Overall survival Kaplan-Meier graph of CCND1 euploid patients (black) and CCND1 amplified patients (red). Survival analysis was performed using Mantel-Cox log rank test with an alpha of 0.05. 
A.

CCND1

Portion 5'UTR

$\wedge$

Length 222nts

Intron 1

1519nts

Intron 2

Intron 4

3'UTR

…...

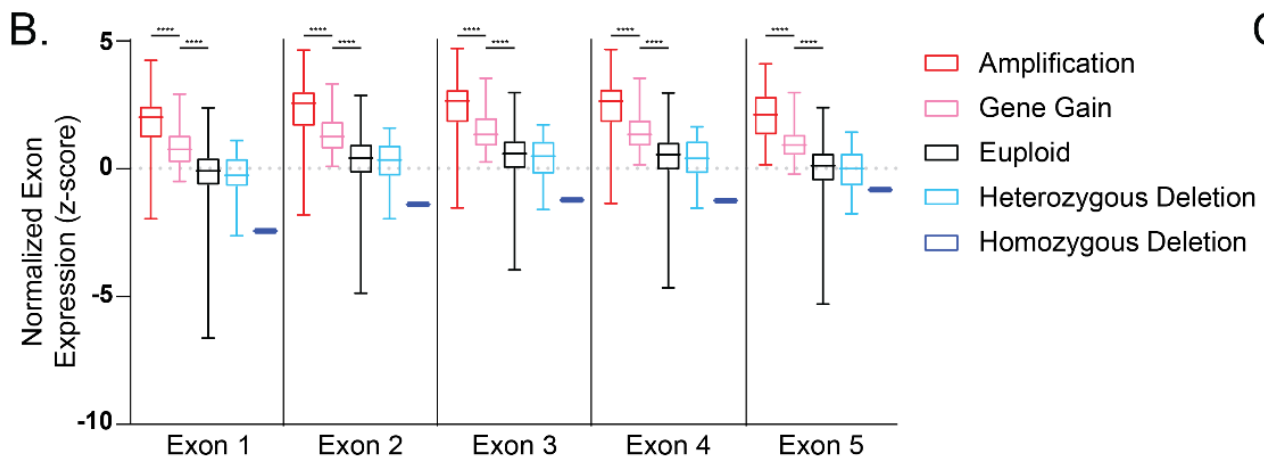

C. 2975nts 165nts 3192nts

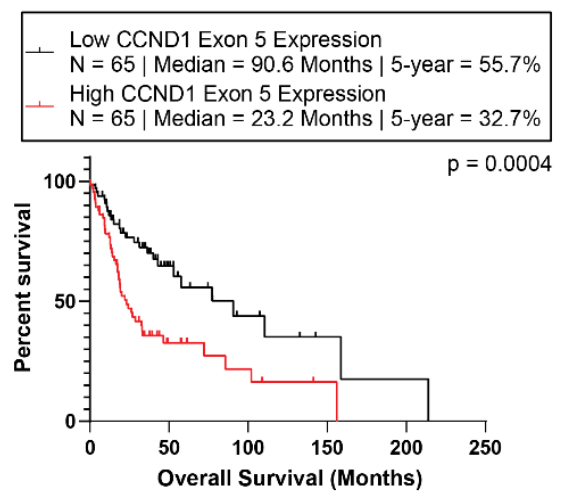

Figure 2. The 3'-UTR of CCND1 is expressed in patients and is associated with poor survival. A. Diagrammatic representation of CCND1 mRNA with UTRs (black), ORFs (yellow) and introns (magenta) locations and lengths. B. Exon-specific RNA expression values normalized to euploid patients (z-score). C. Overall survival Kaplan-Meier graph of low CCND1 exon 5 expression (black) and high CCND1 exon 5 expression (red). Survival analysis was performed using Mantel-Cox log rank test with an alpha of 0.05.
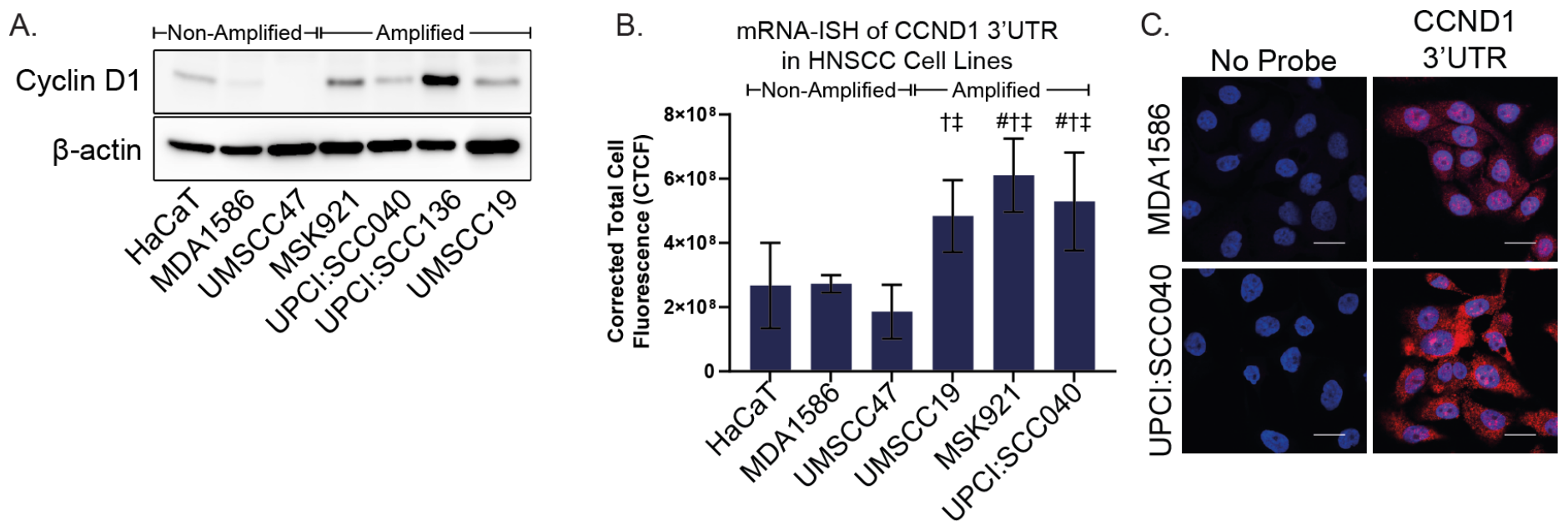

Figure 3. Cyclin D1 protein and mRNA is overexpressed in amplified cell lines and mRNA localizes to

cytosol. A. Western blot of amplified and non-amplified HNSCC cell lines for cyclin D1 and beta-actin. B. Corrected total cell fluorescence (CTCF) of amplified and non-amplified cell lines for CCND13'-UTR mRNA-ISH. \#: $p<0.05$ compared to HaCat; $\uparrow: p<0.05$ compared to MDA-1586; $\ddagger$ : $p<0.05$ compared to UMSCC48. Statistical significance was determined using ANOVA and Tukey's post-hoc with an alpha of 0.05 . C. Representative images of MDA-1586 (non-amplified) and UPCI:SCC040 (amplified) cell line CCND1 3'-UTR mRNA ISH. 


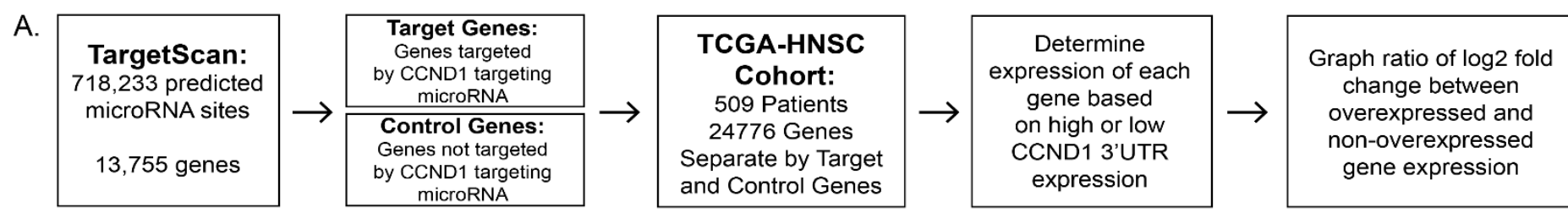

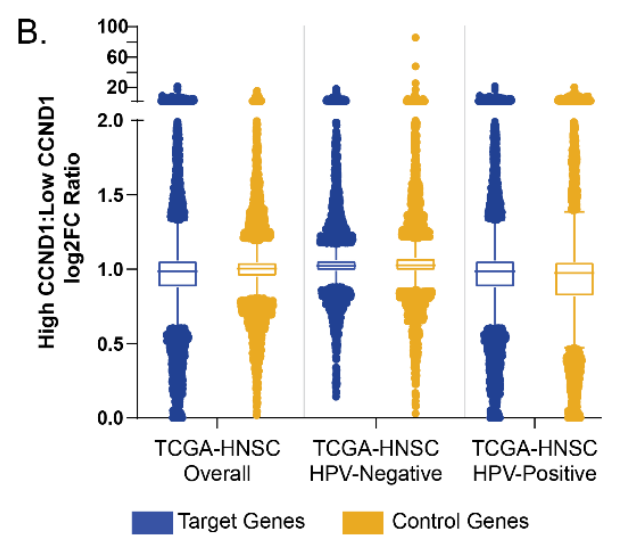

D. "Normal" HNSCC Transcription

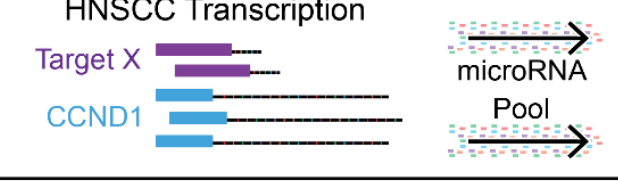

11q13 / CCND1 Amplified HNSCC Transcription
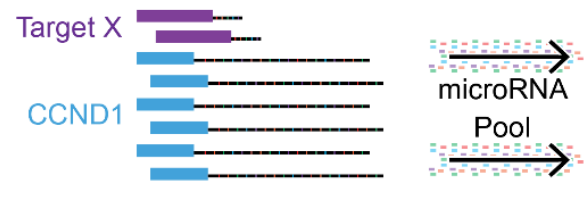

C.
Potential Target Degradation Mechanisms
Potential Translation Inhibition Mechanisms

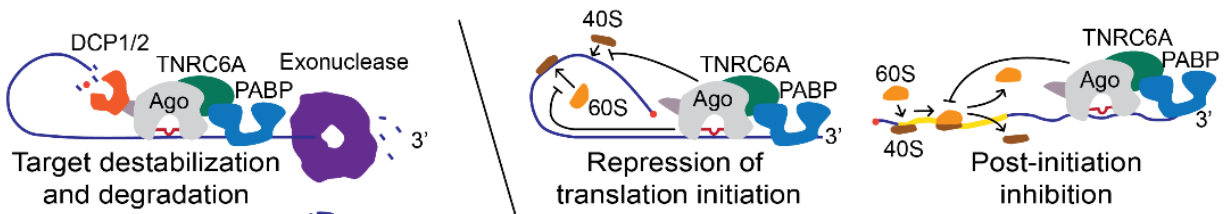
and degradation

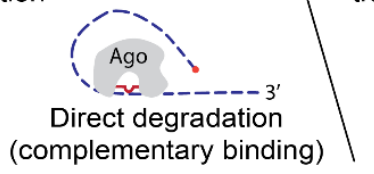

(complementary binding)

Downregulation of target transcripts by degradation or blocking translation

IIIIIIa.

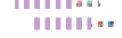

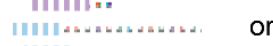

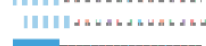

or

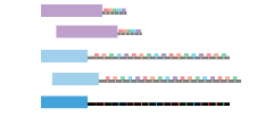

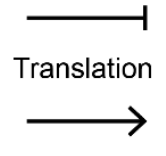

CCND1 ceRNA-mediated de-repression of target expression
Altered Protein Expression inhibition
CCND1

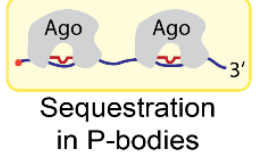

"Normal" Protein Expression

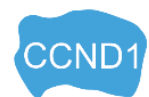

Figure 4. Global mRNA expression is not altered by CCND1 3'-UTR overexpression. A. Project outline describing determination of CCND1 MRE sharing target mRNAs and determination of global mRNA expression. B. Global mRNA expression changes in High CCND1 3'-UTR expression:low CCND1 3'-UTR expression ratio (log2FC) between CCND1 MRE target genes (blue) and control genes (yellow) in TCGA-HNSC overall, HPPnegative, and HPV-positive cohorts. C. Mechanisms of ceRNA-mediated regulation of cellular transcripts include degradive (left) and sequestration mechanisms. D. Proposed mechanism of CCND1 3'-UTR-induced ceRNArelated regulation. 


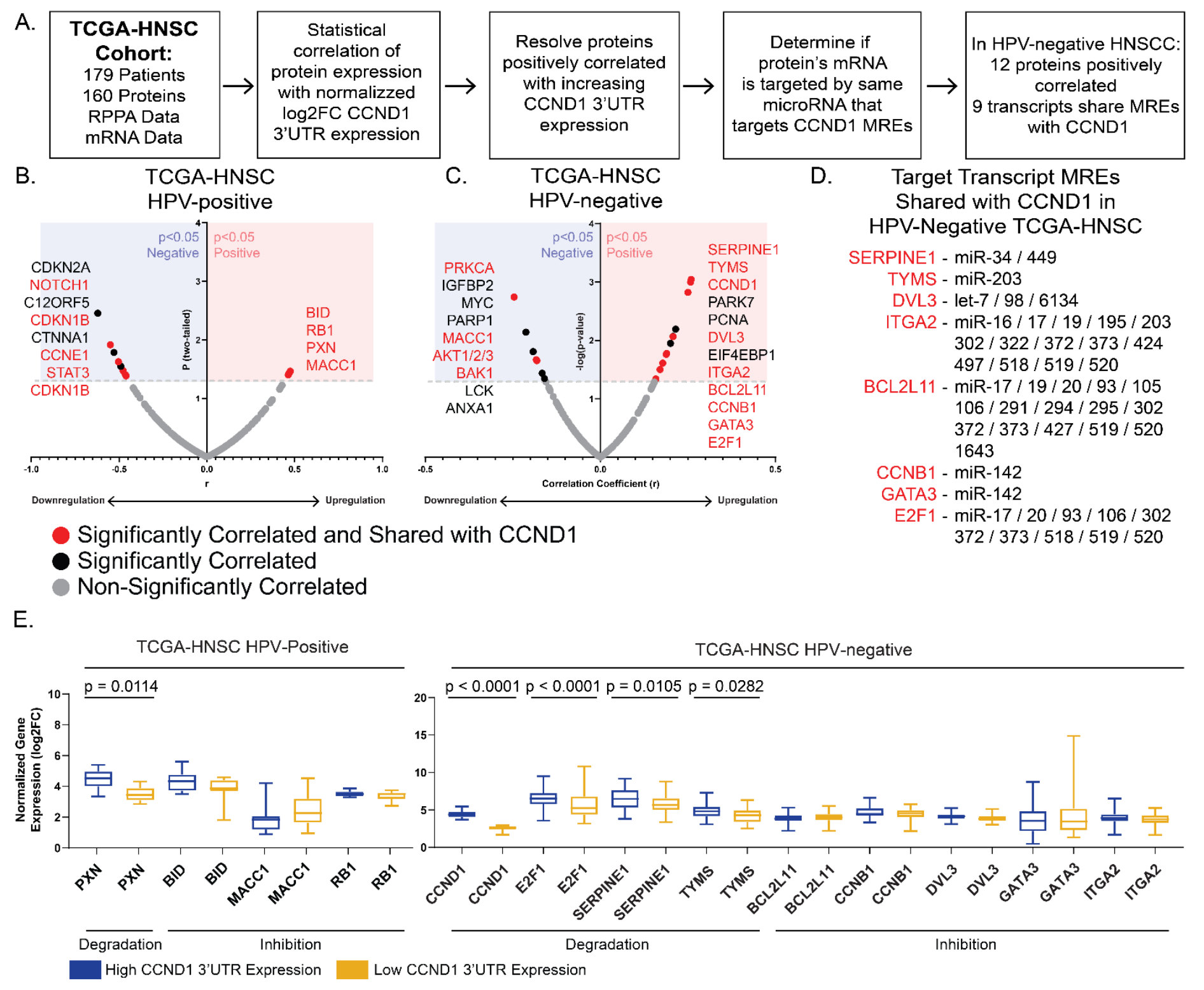

Figure 5. Target protein regulation related to CCND1 3'-UTR expression in TCGA-HNSC. A. Project outline describing determination of CCND1 MRE sharing target mRNAs B-C. Pearson correlation between target protein RPPA value and CCND1 3'UTR log2FC in TCGA-HNSC HPV-positive (B) and HPV-negative (C) patients. Blue boxes represent significantly negatively correlated proteins and red boxes represent significantly positively correlated proteins. Proteins are labeled as non-significantly correlated (grey), significantly correlated (black), or significantly correlated and also sharing CCND1 MREs (red). D. Target protein transcript MREs shared with CCND1 in HPV-negative HNSCC. E. Target protein mRNA expression values (log2FC) stratified by high (blue) and low (yellow) CCND1 3'-UTR expression in HPV-positive (left) and HPV-negative (right) patients. Statistical significance was determined using Pearson correlation or one-way ANOVA and Tukey's post-hoc with an alpha of 0.05 . 
A.

C.

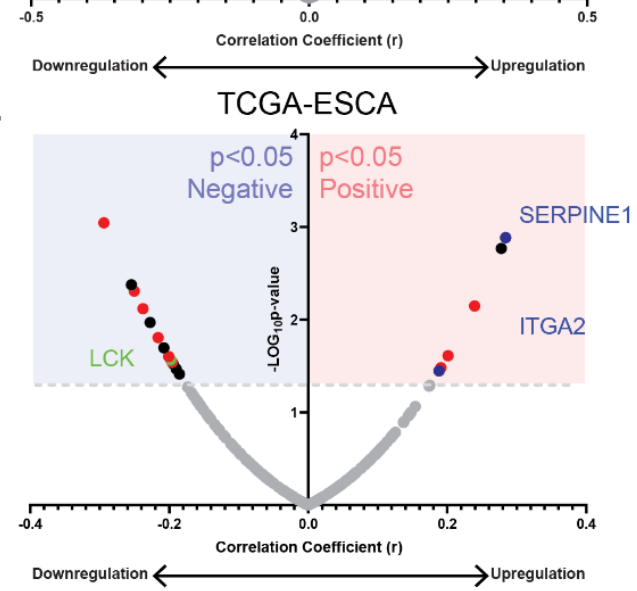

B

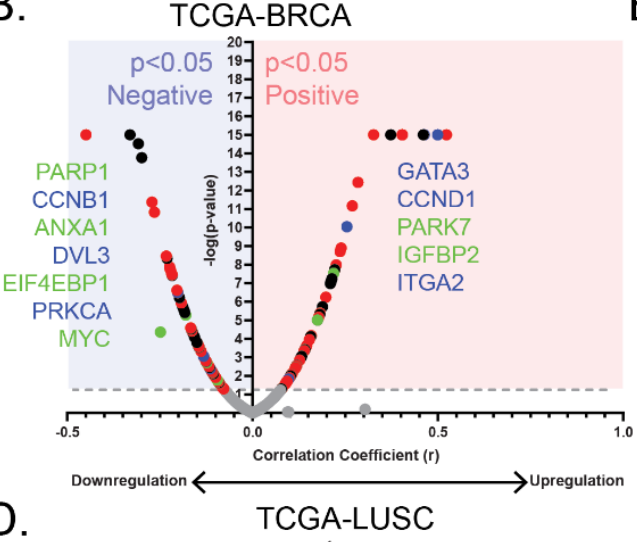

Significantly Correlated and Shared with CCND1 and HNSC

Significantly Correlated and Shared with HNSC

Significantly Correlated and Shared with CCND1

Significantly Correlated

Non-Significantly Correlated
E. Target Proteins shared with TCGA-HNSC

Shared Positive

Correlations

CCND1

ITGA2

PARK7

SERPINE1

GATA3

Shared Negative Correlations

ANXA1

MYC

PARP1

PRKCA

LCK

Discordant

Correlations

BCL2L11

CCNB1

DVL3

EIF4EBP1

IGFBP2

F. TCGA-BRCA

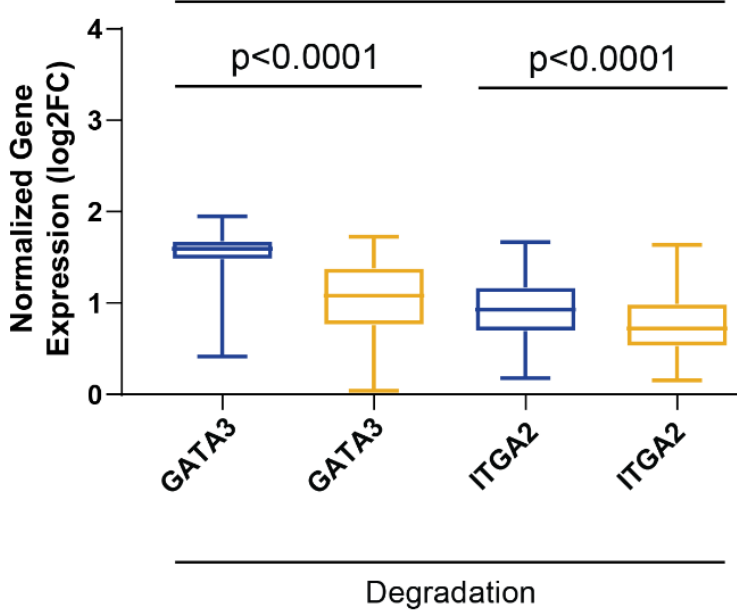

High CCND1 3'UTR Expression

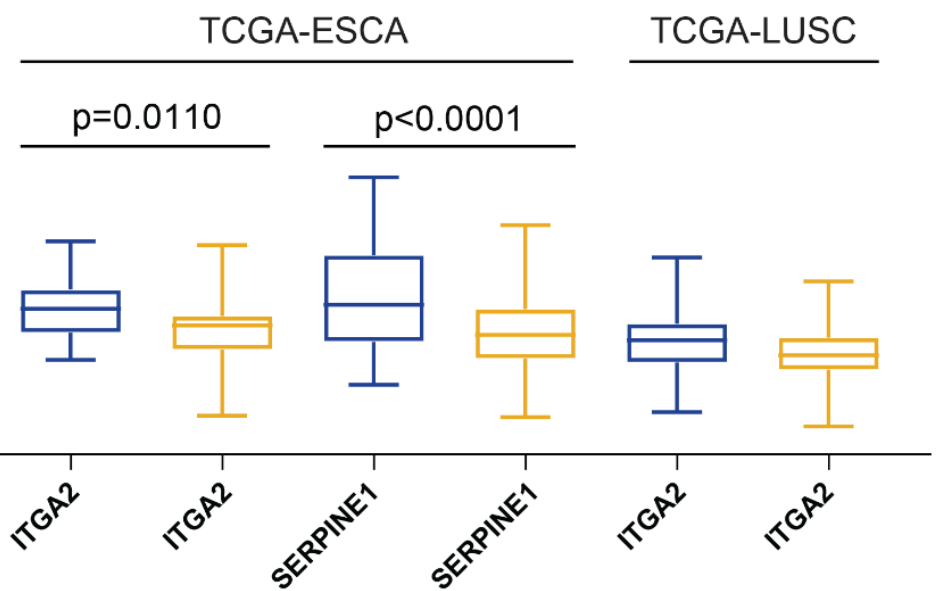

Degradation Inhibition Low CCND1 3'UTR Expression

Figure 6. Target protein regulation related to CCND1 3'-UTR expression in other 11q13-amplified cancers.

A-D. Pearson correlation between target protein RPPA value and CCND1 3'UTR log2FC in TCGA-BLCA (A), TCGA-BRCA (B), TCGA-ESCA (C), and TCGA-LUSC (D) patients. Blue boxes represent significantly negatively 
correlated proteins and red boxes represent significantly positively correlated proteins. Proteins are labeled as non-significantly correlated (grey), significantly correlated (black), significantly correlated and also sharing CCND1 MREs (red), significantly correlated and shared with TCGA-HNSC (green), or significantly correlated and shared with TCGA-HNSC and CCND1 (blue). E. Target protein transcript MREs shared with TCGA-HNSC. Alterations found in TCGA-HNSC are labeled in green and alterations in both TCGA-HNSC and CCND1 are labeled in blue. F. Target protein mRNA expression values (log2FC) stratified by high (blue) and low (yellow) CCND1 3'-UTR expression in TCGA-BRCA, TCGA-ESCA, and TCGA-LUSC patients. Statistical significance was determined using Pearson correlation or one-way ANOVA and Tukey's post-hoc with an alpha of 0.05. 


\section{Study 3 Supplementary Materials: Copy Number Alterations Identify a Smoking-Associated Expression Signature Predictive of Poor Outcome in Head and Neck Squamous Cell Carcinoma}

Brenen W. Papenberg ${ }^{1}$, Ivan Martinez ${ }^{2}$, Peter Stoilov ${ }^{1}$ and Scott A. Weed ${ }^{1 *}$

Departments of ${ }^{1}$ Biochemistry, ${ }^{2}$ Microbiology, Immunology and Cell Biology, Program in Cancer Cell Biology, West Virginia University, Morgantown, West Virginia, 26506

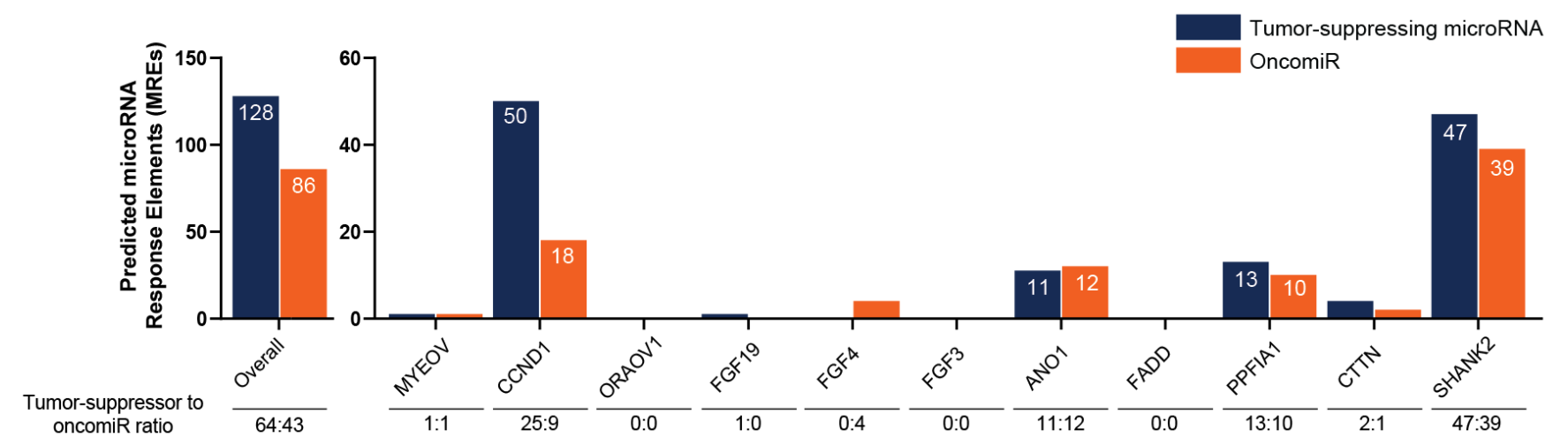

Supplementary Figure S1. Predicted MREs of genes within the 11 q13 amplicon. Predicted tumorsuppressing MREs (blue) and oncomiRs (red) were evaluated for each gene within the 11 q13 amplicon. Calculated tumor-suppressor:oncomiR ratio is displayed below each gene. 


\section{Addendum to Study 3: The CCND1 3'-UTR as a Potential Driver of Poor Outcome in Chromosome 11q13-amplified Head and Neck Squamous Cell Carcinoma}

Brenen W. Papenberg ${ }^{1}$, Ivan Martinez ${ }^{2}$, Peter Stoilov ${ }^{1}$ and Scott A. Weed ${ }^{1 *}$

Departments of ${ }^{1}$ Biochemistry, ${ }^{2}$ Microbiology, Immunology and Cell Biology, Program in Cancer Cell Biology, West Virginia University, Morgantown, West Virginia, 26506

Running title: CCND1 3'-UTR in HNSCC progression

Corresponding Author: *Scott A. Weed, West Virginia University Cancer Institute, P.O. Box 9300, Morgantown, WV 26506, Phone: 304-293-3016, Fax: 304-293-4667, scweed@hsc.wvu.edu.

Keywords: CCND1, competing endogenous RNA, 3'-UTR, microRNA, head and neck cancer 


\section{Background}

In Study 3, we have identified potential roles of CCND13'-UTR in the progression of HNSCC. However, patients that overexpress CCND1 Exon 5 also overexpress CCND1 Exon 1 (Figure 1, Study 3 Figure 2). In this addendum to Study 3, we aim to separate the effects of CCND1 ORF expression and CCND13'-UTR expression on patient survival as well as establish cellular mechanisms of CCND1 3'-UTR ceRNA-mediated effects on observed differences in patient survival utilizing preliminary in vitro cell biology studies. 


\section{Methods}

\section{Cell Lines and Cell Culture}

Cell lines used were MDA1586(1) and UPCI:SCC040(2). All lines were propagated in Dulbecco's Modified Eagle Media (DMEM) supplemented with 10\% fetal bovine serum and 1\% penicillin-streptomycin. Cell lines expressing engineered CCND1 constructs were selected using $10 \mu \mathrm{g} / \mathrm{ml}$ blasticidin (\#A1113903, Gibco). Cell lines stably infected with pLKO.1-puro CCND1 shRNA or scramble control shRNA were generated by $20 \mu \mathrm{g} / \mathrm{ml}$ puromycin (\#P9620, Sigma-Aldrich) selection following standard methods.

\section{Development of CCND1 Constructs}

A plasmid containing an HA-tag at the 3' end of the CCND1 ORF (CCND1-HA) was purchased from Addgene (\#9050). The ORF-HA sequences were PCR amplified to produce a 1,158 bp BamHI-Sall fragment that was then subsequently ligated into pLenti CMV Blast empty (w263-1) (\#17486, Addgene) that was cut using the same restriction enzymes to produce the CCND1-ORF (ORF) construct. A 1,158 bp BamHI-Xhol fragment was made in parallel for ligation to a 3'-UTR fragment and then into pLenti CMV Blast empty. A CCND1 plasmid containing the 3'-UTR(3,4) provided by Kathy Borden (University of Montreal) was PCR amplified to produce a 3,212 bp Xhol-Sall fragment that was ligated to the $3^{\prime}$ end of the 1,158 bp BamHI-Xhol ORF fragment to create the CCND1-FL (FL) construct. The ORF was made refractory to two CCND1 shRNAs (TRCN0000295873 and TRCN0000295874) using a site-directed mutagenesis kit (\#200523, Agilent), following manufacturer's protocol to produce the final ORF and FL constructs. A frameshift mutation was generated by insertion of a guanine base immediately after the ATG start site in the +4 position to create the CCND1-FS (FS) construct. The pLenti CMV Blast empty served as the EV construct.

\section{Western Blotting and Antibodies}

Western blotting was conducted as described(5) and visualized with autoradiography film (E3012, Denville Scientific) or captured by an Amersham Imager 600 (GE Healthcare Bio-Sciences). Antibodies used were anticyclin D1 (sc-718, 1:1000; Santa Cruz Biotechnology), anti-HA-Tag (\#3724, 1:1000; Cell Signaling Technology), anti-GFP (Clontech Labs Living Colors® JL-8), and anti- $\beta$-actin (\#8457, 1:1000; Cell Signaling Technology). 


\section{Quantitative RT-PCR}

Total cellular RNA was extracted with Trizol Reagent (\#15596026, Ambion) following manufacturer's instructions. Eluted RNA was then treated with Turbo DNA-free kit (\#AM1907, Invitrogen) to remove DNA. Concentrations of RNA were evaluated using a Nanodrop 2000 Spectrophotometer. $1 \mu \mathrm{g}$ of total RNA was converted to cDNA using the iScript cDNA synthesis kit (\#1708890, Bio-Rad). RT-qPCR was performed using the SsoAdvanced Universal SYBR Green Supermix (\#1725271, Bio-Rad) and primers specific to the 3'-UTR of CCND1. Relative expression was calculated using the $\triangle \triangle C T$ method using GAPDH as a control endogenous gene.

\section{RNA In-Situ Hybridization}

RNA in-situ hybridization was performed as previously described(6). Briefly, custom Stellaris® FISH Probes were designed against CCND1 3'UTR by utilizing the Stellaris ${ }^{\circledR}$ RNA FISH Probe Designer (Biosearch Technologies, Inc., Petaluma, CA) available online at www.biosearchtech.com/stellarisdesigner (Version 2017). The CCND1 3'UTR were conjugated to Cy5 for fluorescent visualization. Cells were grown on coverglass, permeabilized and hybridized with a buffer containing the labeled CCND1 probes following the manufacturer's instructions available online at www.biosearchtech.com/stellarisprotocolscells. Cells were counterstained with DAPI to visualize nuclei and mounted onto slides for microscopic analysis using a Nikon A1R confocal microscope. Corrected total cell fluorescence was calculated for each cell line. Statistical significance was determined one-way ANOVA and Tukey's post-hoc with an alpha of 0.05 .

\section{Cell Cycle Analysis}

MDA-1586 cells were grown to confluence and maintained for five days in order to induce contact-inhibitionmediated cell synchronization. (https://www.future-science.com/doi/pdf/10.2144/01306rv01). UPCI:SCC040 cells were synchronized using a double thymidine block as previously described (https://pubmed.ncbi.nlm.nih.gov/5165443/). Cells were then fixed and stained with propidium iodide (PI) as previously described $(7,8)$ before flow cytometry analysis. Technical triplicates of both MDA-1586 and UPCI:SCC040, as well as biological triplicates of UPCI:SCC040 were assessed by flow cytometry. Flow cytometry experiments were performed in the West Virginia University Flow Cytometry \& Single Cell Core 
Facility, which is supported by the National Institute of Health grant U51 GM104942 (WV CTSI) and the Institutional Development Awards (IDeA) from the National Institute of General Medical Sciences of the National Institutes of Health grant numbers P30GM121322 (TME CoBRE) and P20GM103434 (INBRE). Data were analyzed using FCS Express 6 (De Novo Software). 


\section{Results}

\section{Exogenous overexpression of CCND1 constructs in HNSCC cell lines}

To delineate effects of the CCND1 ORF and CCND1 3'-UTR on cancer progression, multiple CCND1 constructs were generated (Figure 2A). An empty vector (EV) control plasmid of pLenti Blast was used as a backbone for the cloning of $C C N D 1$ sequences and also serves as a control for downstream studies. A construct expressing green fluorescent protein (GFP) was generated by cloning GFP into EV to generate a control cell line to serve as a control in downstream experimentation. The CCND1-HA ORF was mutated using site-directed mutagenesis to form a CCND1-HA ORF refractory against two different CCND1-targeting shRNAs designed to knockdown of endogenous CCND1 protein expression. This CCND1-HA ORF was then cloned into EV to form the ORF construct in order to evaluate effects of CCND1-HA ORF alone. The full-length CCND13'-UTR was subsequently cloned into ORF to generate the full-length CCND1-HA sequence (FL). This construct was subsequently mutated at the +4 position by to introduce a frameshift that ablates expression of exogenous cyclin D1 protein $(F S)$ to evaluate overexpression of CCND1 3'-UTR alone without loss of mRNA secondary structure that may be required for ceRNA effects.

These constructs were introduced into the CCND1-diploid HNSCC cell line MDA-1586 using lentiviral infection to evaluate gain-of-function effects via CCND1 overexpression. Successful transduction and construction expression were evaluated by western blotting, RT-qPCR, and mRNA-FISH depending on produced protein and/or mRNA products. Western blot visualization displayed successful stable expression of CCND1 constructs with the presence of the HA epitope as a band of increased molecular weight reacting with to and anti-cyclin D1 in the MDA-1586-ORF and MDA-1586-FL cell lines (Figure 2B). Since overexpression of 3'-UTR cannot be assessed by western blot due to lack of protein production, RT-qPCR was utilized to visualize expression of CCND1 3'-UTR in MDA-1586 cell lines. Both lines showed increased relative expression of CCND1 3'-UTR in MDA-1586-FL and MDA-1586-FS cell lines (Figure 2C). To further assess the expression of CCND1 constructs in MDA-1586 cells, mRNA-FISH was utilized which also allowed assessment of sub-cellular location of CCND1 mRNA. Microscopic analysis indicated overexpression of CCND1 mRNA constructs in the cytosol of MDA-1586 cell lines (Figure 2D). 
To account for potential oncogene-addiction introduced through $11 \mathrm{q} 13$ amplification and subsequent enhanced endogenous CCND1 overexpression, the engineered CCND1 constructs were introduced into the 11q13amplified HNSCC cell line UPCI:SCC040. Visualization of protein expression by western blotting indicated successful overexpression of the ORF and FL CCND1 constructs (Figure 2E). To test the ability of these constructs to resist shRNA knockdown, UPCI:SCC040 cells expressing ORF, FL and ATG? were stably infected with an anti-CCND1 shRNA. Western blot visualization revealed successful knockdown of endogenous CCND1 without impacting recombinant CCND1 overexpression (Figure 2F).

\section{Exogenous CCND1 3'-UTR overexpression does not appear to alter HNSCC cell cycle progression}

Due to the role of cyclin D1 in the progression of cell cycle, we sought to investigate the effects of overexpression of the CCND13'-UTR on cell cycle to assess 3'-UTR-mediated effects. Cell cycle was assessed using PI staining of synchronized HNSCC cell lines. MDA-1586 gain-of-function analysis displayed a modest increase of cells in the G2-M phase, suggesting increased proliferation of cells overexpressing the FL or FS constructs (Figure 3A). However, MDA-1586 cells were synchronized using a non-conventional method and the experimental results are only one run of analysis at only one time point (24 hours). Further analysis was conducted using the UPCISCC:040 cell, due to the aforementioned potential for oncogene addiction in the 11q13-amplified background that maybe necessary to visualize any changes in CCND1-amplified cells. Double thymidine block synchronization followed by biological triplicate (each repeat containing technical triplicates) revealed no differences in cell cycle at 6,12 , or 24 hours in transfected UPCISCC:040 cells (Figure 3B). This suggests that CCND1 3'-UTR overexpression does not impact HNSCC progression by modulating increase in cell proliferation or changes in cell cycle kinetics. 


\section{Future Directions}

In Study 3, we identified differentially expressed proteins that were correlated with CCND1 3'-UTR expression changes. In this Addendum, we show that CCND1 ceRNA-mediated effects on tumor progression do not act through increased cell cycle progression. However, the target proteins identified in Study 3 may function through other tumor-enhancing mechanisms, including increased proliferation, invasion, migration, apoptosis resistance, and DNA synthesis. Other assays such as BrdU incorporation or further optimized PI staining assays may be able to better evaluate potential changes on the cell cycle. These possibilities, coupled with the survival differences in patients with high CCND1 3'-UTR expression, underscore the need for further studies. The different $C C N D 1$ expression constructs provide useful tools for the evaluation of any ceRNA-mediated effects in HNSCC cell lines in future work.

While TCGA offers a useful tool for evaluating global mRNA expression changes, evaluation using CCND1 expression vectors is still vital for understanding CCND1 ceRNA-mediated effects on mRNA. For determining transcriptional changes specific to the CCND1 3'-UTR, isolated RNA from UPCISCC:040 parental and EV, FL, ORF and FS overexpressing cells will be DNAse and RiboZero treated before RNAseq in 2x150 format at 50M reads. Data will be evaluated for differential gene expression(9). Highly upregulated transcripts shared by FL and FS overexpressing cells compared with control and ORF lines will be considered novel candidates for CCND1 ceRNA activity. 3'-UTR regions from candidate targets will be evaluated by DIANA Tools, TargetScan and the literature for shared microRNA response elements (MREs) with the CCND1 3'-UTR. As TCGA results suggest, evaluation of target proteins may be more useful than purely transcriptomic analysis. To evaluate changes in protein expression, cells will be lysed with the EasyPrep Mini MS prep kit (Fisher) and subjected to label-free quadrupole Orbitrap liquid chromatography tandem mass spectroscopy (LC-MS/MS)(10) with the assistance of the WVU BioNano Research Facility. SIEVE, Compound and Proteome Discoverer will be used for peptide identification, protein assembly and quantification(11). Relative protein values will be compared for FL and FS overexpressing cells to control and ORF cells, with overexpressed proteins in FL and FS cells evaluated for MREs shared with the CCND1 3'-UTR. We anticipate multiple transcripts and proteins will be upregulated in the FS and FL cell lines compared to controls, including those identified in patient samples (Study 
3 Fig. 5D). Upregulated proteins in FL cells may be due to cyclin D1 protein overexpression, rather than ceRNA by the 3'-UTR. These cases will be compared with the FS results.

To test the ability of the CCND1 3'UTR as a ceRNA for putative target expression, the 3'-UTR regions for candidate and discovered ceRNA targets will be subcloned into a luciferase reporter vector and assayed(12). Constructs will be stably transfected into HNSCC CCND1 KD cells with overexpression of EV, ORF, FL and FS transcripts (Figure 2A). Target-driven luciferase activity will be measured. To determine if target overexpression is dependent on CCND1 ceRNA activity, the established seed region of MREs for miRNAs in each respective target 3'-UTR (Fig. 6D) will be mutated. Knockdown cells expressing miRNA-mutant constructs (MM) will be generated and the activity of the target 3'-UTR luciferase reporter compared to the CCND1 ORF, FL and FS control cells. These results will be validated using target site blocking (TSB) oligonucleotides (Qiagen miRCURY) against the 3'-UTR MREs for each target. TSBs will be transfected into control ORF, FL and FS cells, and target protein and mRNA expression will be measured. To control for specificity, TSBs will be transfected into MM cells and assayed for target expression. We predict overexpression of CCND1 FL and FS to increase candidate ceRNA target expression compared to ORF constructs due to 3'-UTR titration of shared miRNAs. TSB transfection is expected to block CCND1 and target transcript MREs, producing an inhibitory effect.

Proliferation will be evaluated using CyQuant NF (Fisher) and Ki67 immunolabeling of fixed cells. Anchorage independent growth will be assayed for colony formation in soft agar as described(13). Apoptosis will be evaluated using annexin V staining and quantified by flow cytometry. HNSCC invasiveness will be evaluated with each cell line using an organotypic assay as described(14,15). Degree and extent of invasion will be quantified using a calculated index based on histologic imaging of H\&E stained sections using Metamorph software as previously detailed(16). MM cell lines with known ceRNA effects for candidate targets will be evaluated in accordance with appropriate assays, with target expression monitored by western blotting and RTqPCR. FL and FS overexpression are anticipated to effect one or more hallmarks to a greater extent than ORF and EV. The FS construct may not enhance all of these processes in some cases due to lack of coincident cyclin D1 protein expression, but will provide additional evidence that the 3'-UTR contributes to one or more aspects of HNSCC progression. 
$11 q 13$ amplification and CCND1 overexpression occurs in other tobacco-associated malignancies(17-20). Correlation analyses identify several shared overexpressed candidate CCND1 3'-UTR ceRNA targets (e.g., SERPINE1 and ITGA2) (Study 3 Figure 6), further underscoring the significance of the proposed work. The outcome of these future directions addressing regulation of the miRNA network by CCND1 transcript overexpression will provide unique insights into the consequence of 11q13 amplification and associated poor outcomes in HNSCC. 


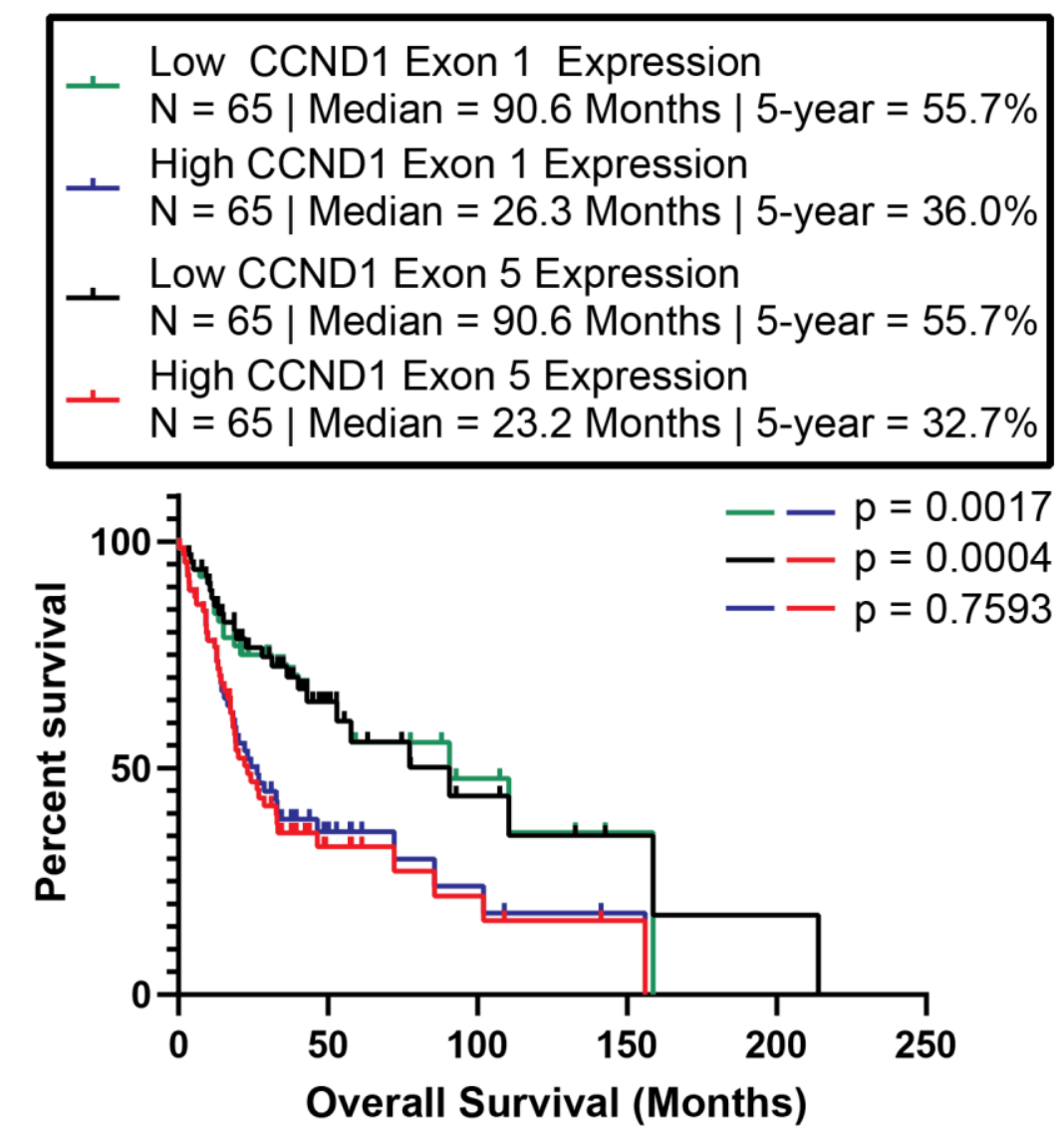

Figure 1. Patient overall survival stratified by CCND1 exon expression. Survival analysis of patients with high CCND1 exon 1 (blue) or exon 5 (red) overexpression $(\mathrm{N}=65)$ or low exon 1 (green) exon 5 (black) expression ( $\mathrm{N}=65)$ using the Broad HNSC Level 3 exon expression dataset. Z-scores were normalized to diploid values. Survival analysis was performed using Mantel-Cox log rank test with an alpha of 0.05 . 
A.

\begin{tabular}{|c|c|c|c|}
\hline $\mathrm{EV}$ & CMV & & \\
\hline GFP & CMV & GFP & \\
\hline ORF & CMV & & $\mathrm{HA}$ \\
\hline $\mathrm{FL}$ & $\mathrm{CMV}$ & & HA CCND1 3'UTR \\
\hline FS & CMV & & HA CCND1 3'UTR \\
\hline
\end{tabular}
shRNA Refractory CCND1 ORF Mutation

B.

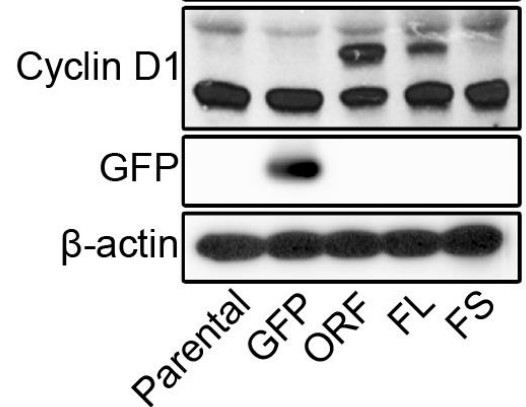

D.

MDA1586

MDA1586

MDA1586

MDA1586

MDA1586

$$
\text { Parental }
$$

GFP
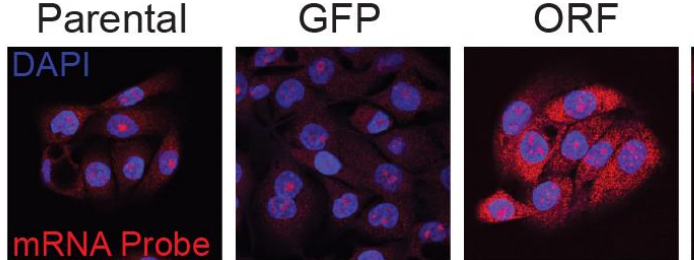

$\mathrm{FL}$

ORF

Probe
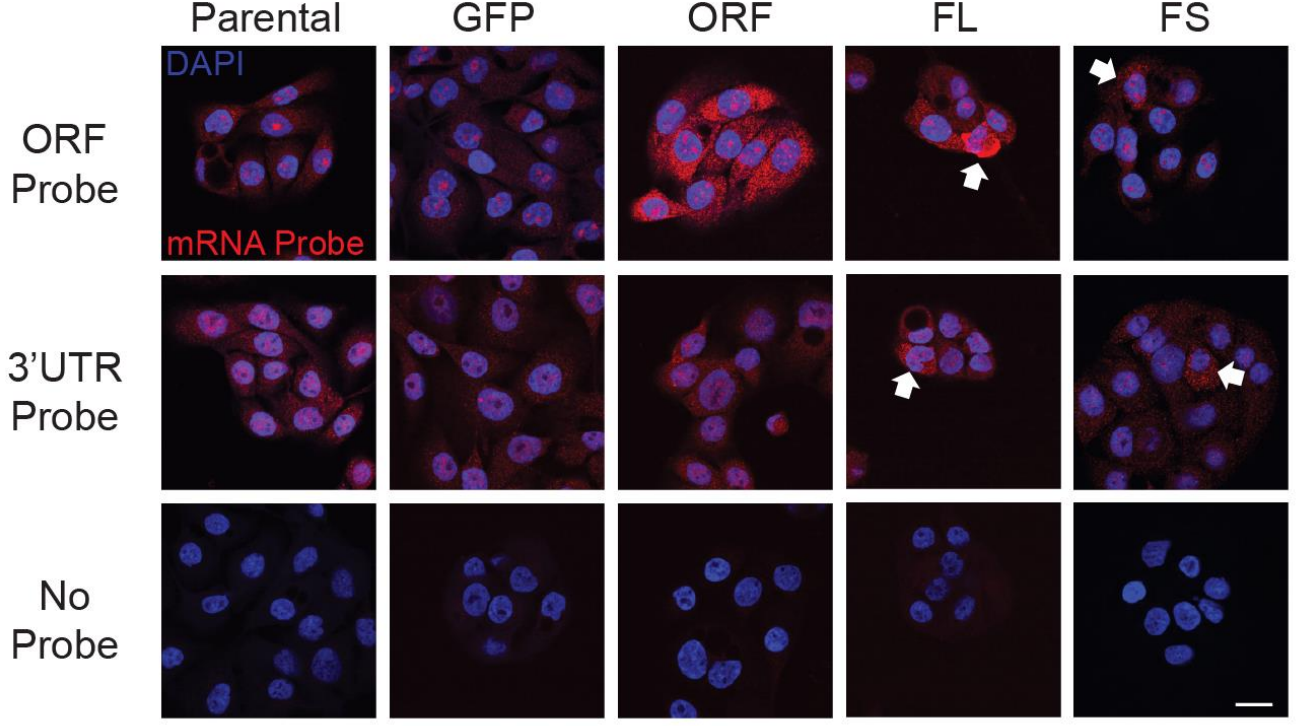

C. $\begin{gathered}\text { RT-qPCR Analysis of } \\ \text { CCND1 } 13 \text { 'UTR in MDA-158 }\end{gathered}$

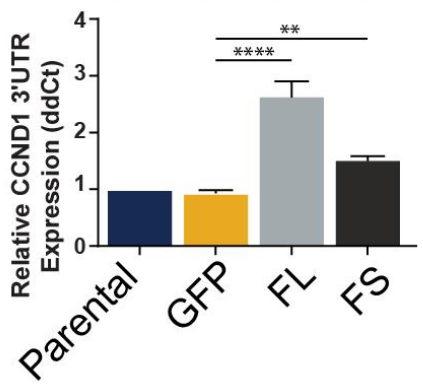

E.

Cyclin D1
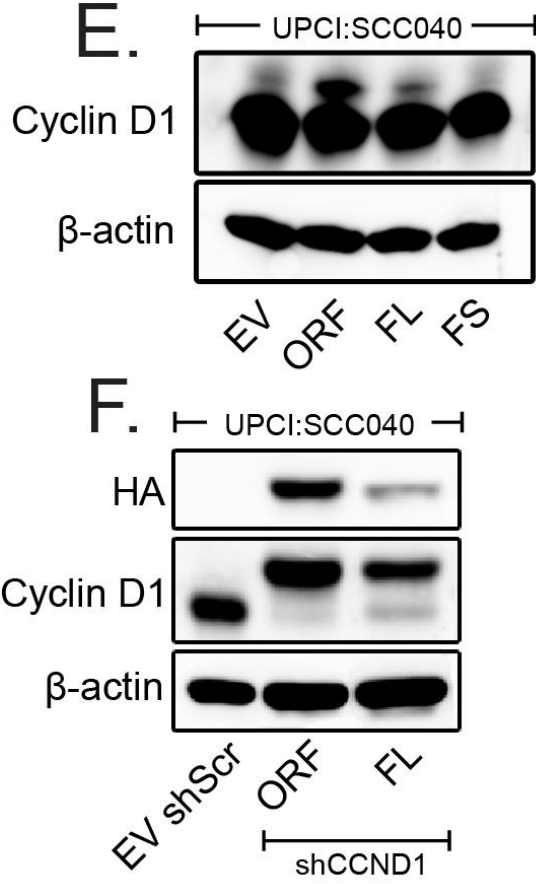

Figure 2. Development of CCND1 constructs and expression in HNSCC cell lines. A. CCND1 expression constructs subcloned into pLenti-Blast CMV (Addgene). EV; empty vector control. GFP; GFP control. ORF; cyclin D1 open reading frame. FL; complete CCND1 transcript with ORF and 3'UTR. FS; point mutant frame-shifted at position +4 in the ORF. B. Protein expression of CCND1 constructs. Western blotting of lysates from disomic MDA-1586 HNSCC cells transduced with the constructs in (A) and blotted as indicated. C. Ectopic expression of CCND1 3'-UTR regions. RNA from the indicated cells was amplified by RT-qPCR using primers targeting the CCND1 3'-UTR. Bars represent mean with SD error bars. Statistical significance was determined using ANOVA and Tukey's post-hoc with an alpha of $0.05 .{ }^{* * *}: p<0.0001 ;{ }^{* *}: p<0.01$. D. Representative images of RNA-FISH for non-amplified MDA-1586 and amplified UPCI:SCC40 HNSCC lines. CCND1 transcripts display abundant cytoplasmic localization to serve as ceRNA. Bar $=20 \mu \mathrm{m}$. E. Protein expression of CCND1 constructs. Western 
blotting of lysates from amplified UPCI:SCC040 HNSCC cells transduced with the constructs in (A) and blotted as indicated. F. CCND1 knockdown and re-overexpression of HA-tagged ORF and FL constructs in $11 \mathrm{q} 13$ amplified UPCI:SCC040. EV; empty vector sh Sramble control.

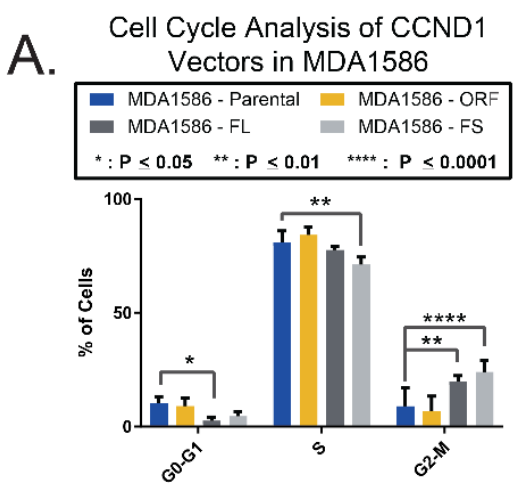

24 Hours

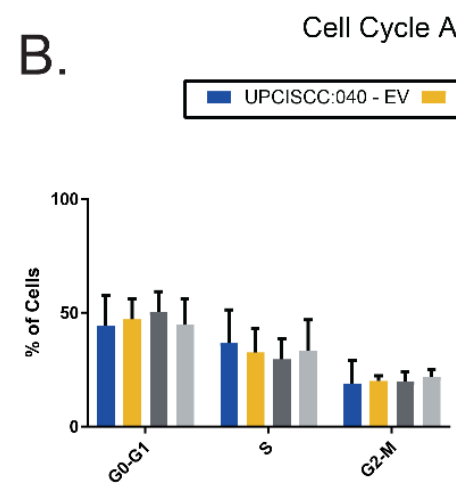

6 Hours

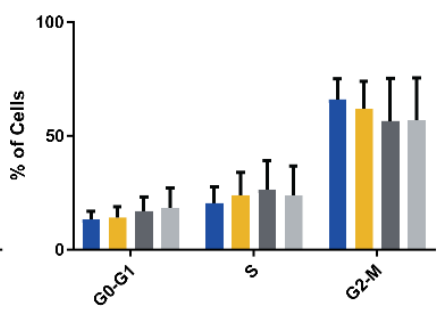

12 Hours

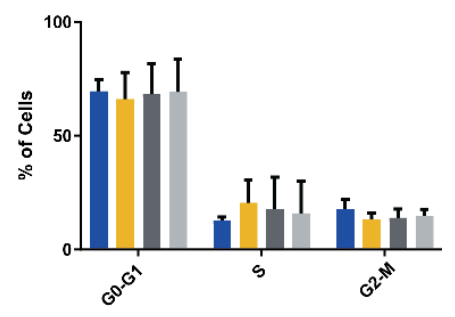

24 Hours

Figure 3. Cell cycle analysis of CCND1 constructs in HNSCC cell lines. A. MDA-1586 and B. UPCISCC:040 CCND1 overexpressing lines were synchronized, PI labeled and sorted by FACS. Analysis was conducted using FCS Express 5. Statistical significance was determined using ANOVA and Tukey's post-hoc with an alpha of 0.05 . 


\section{Bibliography}

1. Markwell SM, Ammer AG, Interval ET, Allen JL, Papenberg BW, Hames RA, et al. Cortactin phosphorylation by casein kinase 2 regulates actin-related protein $2 / 3$ complex activity, invadopodia function, and tumor cell invasion. Mol Cancer Res [Internet]. 2019 [cited 2020 Jul 2];17(4):987-1001. Available from: https://www.ncbi.nlm.nih.gov/pmc/articles/PMC6445698/

2. White JS, Weissfeld JL, Ragin CCR, Rossie KM, Martin CL, Shuster M, et al. The influence of clinical and demographic risk factors on the establishment of head and neck squamous cell carcinoma cell lines. Oral Oncol [Internet]. 2007 [cited 2020 Jul 20];43(7):701-12. Available from:

https://pubmed.ncbi.nlm.nih.gov/17112776/

3. Culjkovic B, Topisirovic I, Skrabanek L, Ruiz-Gutierrez M, Borden KLBB. elF4E promotes nuclear export of cyclin D1 mRNAs via an element in the 3'UTR. J Cell Biol [Internet]. 2005 Apr 25 [cited 2016 Mar 16];169(2):245-56. Available from: https://www.ncbi.nlm.nih.gov/pmc/articles/PMC2171863/

4. Azzi L, Meijer L, Ostvold AC, Lew J, Wang JH. Purification of a 15-kDa cdk4- and cdk5-binding protein. J Biol Chem. 1994;269(18):13279-88.

5. Ammer AG, Kelley LC, Hayes KE, Evans J V, Lopez-Skinner LA, Martin KH, et al. Saracatinib Impairs Head and Neck Squamous Cell Carcinoma Invasion by Disrupting Invadopodia Function. J Cancer Sci Ther [Internet]. 2009 Nov 30 [cited 2020 Jul 20];01(02):052-61. Available from: https://pubmed.ncbi.nlm.nih.gov/20505783/

6. Coassin SR, Orjalo A V., Semaan SJ, Johansson HE. Simultaneous Detection of Nuclear and Cytoplasmic RNA Variants utilizing stellaris ${ }^{8}$ RNA fluorescence in situ hybridization in adherent cells. Methods Mol Biol [Internet]. 2014 [cited 2020 Jul 20];1211:189-99. Available from: https://pubmed.ncbi.nlm.nih.gov/25218386/

7. Shapiro H. Practical Flow Cytometry. 2nd ed. New York: Alan R. Liss, Inc; 1988. 353 p.

8. Darzynkiewicz Z. Nucleic Acid Analysis. In: Robinson J, editor. Current Protocols in Cytometry. J Wiley \& Sons, Inc; 1997. 
9. Damron FH, Oglesby-Sherrouse AG, Wilks A, Barbier M. Dual-seq transcriptomics reveals the battle for iron during Pseudomonas aeruginosa acute murine pneumonia. Sci Rep [Internet]. 2016 Dec 16 [cited 2020 Aug 9];6(1):39172. Available from: http://www.nature.com/articles/srep39172

10. Han X, Aslanian A, Yates JR. Mass spectrometry for proteomics [Internet]. Vol. 12, Current Opinion in Chemical Biology. NIH Public Access; 2008 [cited 2020 Aug 9]. p. 483-90. Available from: http://www.ncbi.nlm.nih.gov/pubmed/18718552

11. Schmidt A, Forne I, Imhof A. Bioinformatic analysis of proteomics data [Internet]. Vol. 8, BMC systems biology. BioMed Central; 2014 [cited 2020 Aug 9]. p. S3. Available from: http://bmcsystbiol.biomedcentral.com/articles/10.1186/1752-0509-8-S2-S3

12. Sharma SB, Lin C-C, Farrugia MK, McLaughlin SL, Ellis EJ, Brundage KM, et al. MicroRNAs 206 and 21 Cooperate To Promote RAS-Extracellular Signal-Regulated Kinase Signaling by Suppressing the Translation of RASA1 and SPRED1. Mol Cell Biol [Internet]. 2014 Nov 15 [cited 2020 Aug 9];34(22):4143-64. Available from: http://www.ncbi.nlm.nih.gov/pubmed/25202123

13. Madajewski B, Boatman MA, Chakrabarti G, Boothman DA, Bey EA. Depleting tumor-NQO1 Potentiates Anoikis and Inhibits Growth of NSCLC. Mol Cancer Res [Internet]. 2015 Jan [cited 2020 Aug 9];43(3):762-73. Available from: http://www.ncbi.nlm.nih.gov/pubmed/26553038

14. Kalabis J, Wong GS, Vega ME, Natsuizaka M, Robertson ES, Herlyn M, et al. Isolation and characterization of mouse and human esophageal epithelial cells in 3D organotypic culture. Nat Protoc [Internet]. 2012 Jan 12 [cited 2020 Aug 9];7(2):235-46. Available from:

http://www.ncbi.nlm.nih.gov/pubmed/22240585

15. Le Bras GF, Allison GL, Richards NF, Ansari SS, Washington MK, Andl CD. CD44 upregulation in Ecadherin-negative esophageal cancers results in cell invasion. Marcus Al, editor. PLoS One [Internet]. 2011 Nov 1 [cited 2020 Aug 9];6(11):e27063. Available from: https://dx.plos.org/10.1371/journal.pone.0027063

16. Gaggioli C, Hooper S, Hidalgo-Carcedo C, Grosse R, Marshall JF, Harrington K, et al. Fibroblast-led 
collective invasion of carcinoma cells with differing roles for RhoGTPases in leading and following cells.

Nat Cell Biol [Internet]. 2007 Dec 25 [cited 2020 Aug 9];9(12):1392-400. Available from:

http://www.nature.com/articles/ncb1658

17. Mori M, Tokino T, Yanagisawa A, Kanamori M, Kato Y, Nakamura Y. Association between chromosome 11 q13 amplification and prognosis of patients with oesophageal carcinomas. Eur J Cancer [Internet]. 1992 Apr 1 [cited 2020 Aug 9];28(4-5):755-7. Available from: https://linkinghub.elsevier.com/retrieve/pii/095980499290109F

18. Xu J, Tyan T, Cedrone E, Savaraj N, Wang N. Detection of 11q13 amplification as the origin of a homogeneously staining region in small cell lung cancer by chromosome microdissection. Genes Chromosom Cancer [Internet]. 1996 Nov 1 [cited 2020 Aug 9];17(3):172-8. Available from: http://doi.wiley.com/10.1002/\%28SICI\%291098-2264\%28199611\%2917\%3A3\%3C172\%3A\%3AAIDGCC5\%3E3.0.CO\%3B2-1

19. Brookes S, Alistair Lammie G, Dickson C, Peters G, Schuuring E. Linkage map of a region of human chromosome band 11q13 amplified in breast and squamous cell tumors. Genes, Chromosom Cancer [Internet]. 1992 Jun 1 [cited 2020 Aug 9];4(4):290-301. Available from:

http://doi.wiley.com/10.1002/gcc.2870040404

20. Proctor AJ, Coombs LM, Cairns JP, Knowles MA. Amplification at chromosome 11q13 in transitional cell tumours of the bladder. Oncogene [Internet]. 1991 May 1 [cited 2020 Aug 9];6(5):789-95. Available from: http://www.ncbi.nlm.nih.gov/pubmed/2052357 


\section{General Discussion:}

This dissertation describes studies that illuminate the relationship between Appalachian HNSCC survival, tobacco-induced DNA damage, and novel functions of the tumor progression driver cyclin D1. The first Study details how increased tobacco exposure in the Appalachian region can contribute to decreased survival of AJCC stage IV HNSCC patients with primary tumors within the HPV-associated oropharynx. The second Study establishes large-scale chromosomal damage that is correlated with increasing patient pack-year value and identifies several candidate genes of unknown or unappreciated function in HNSCC for future studies. The third Study outlines a potential ceRNA-driven mechanism of cyclin D1, a well-known tumor progression driver in HNSCC while the Addendum to Study 3 explores this potential mechanism in vitro. Together, these studies expand on the observed HNSCC disparity within Appalachia, correlate tobacco-use to $11 q 13$ amplification and subsequent overexpression of a subset of genes, and link an 11q13 gene to novel functions (Figure 1). Results from this dissertation could impact patient treatment regimens and clinical trial stratification by solidifying tobacco-use and 11q13 gene, including CCND1, expression levels as important diagnostic criteria.

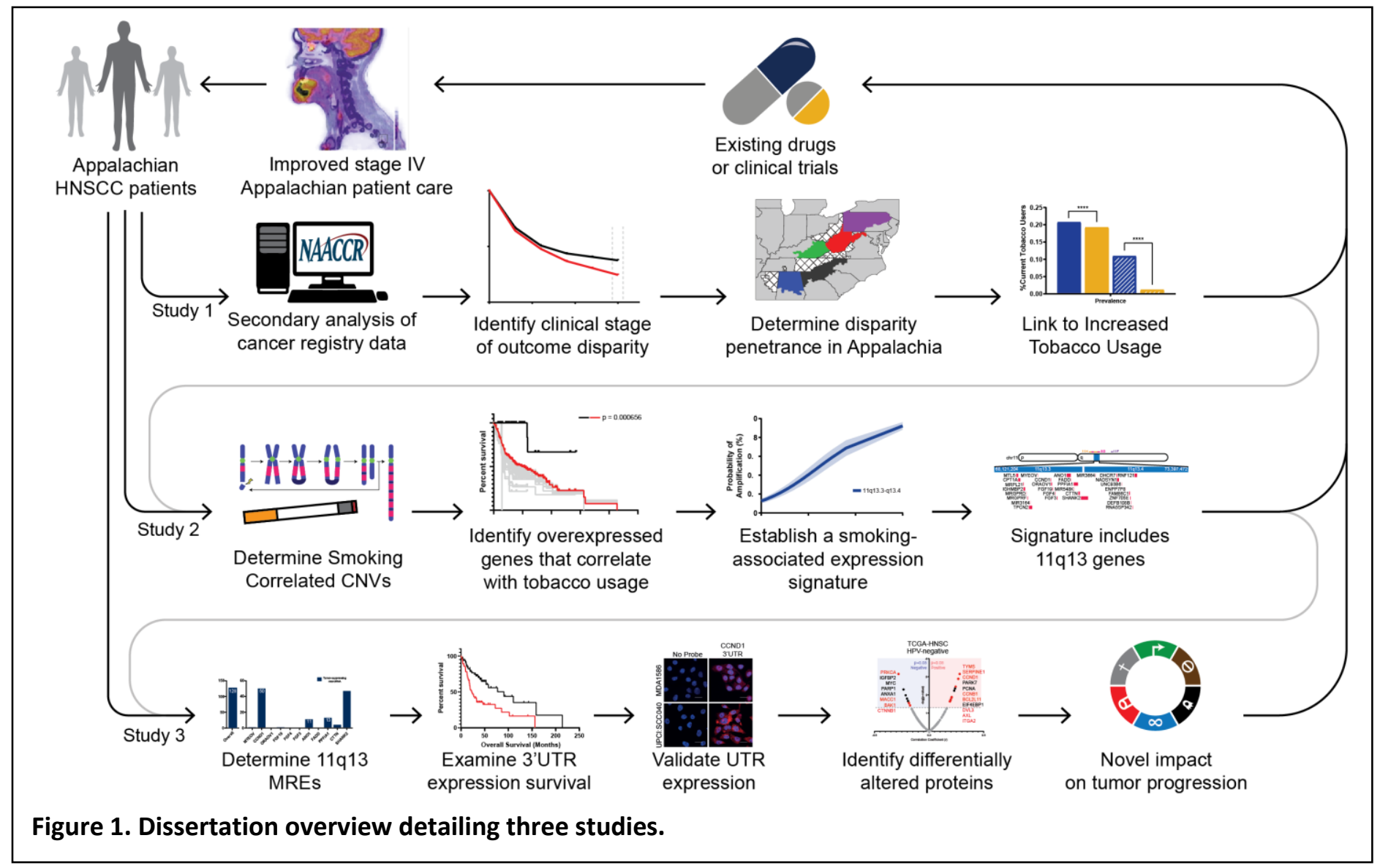


Although oral cavity and pharyngeal cancer incidence and mortality disparities in Appalachia have been documented in the past $(1,2)$, the work outlined in Study 1 further describes these disparities by separating patients by AJCC stage, sex, race, and primary site. Thus, this approach utilizes a comprehensive methodology that includes cancer-specific survival data from the majority of Appalachian counties and the non-Appalachian counties within the same states. The results from this work are definitive descriptions of oral cavity and pharyngeal survival in the Appalachian region, pinpointing the observed disparity to AJCC-6 stage IV white males with tumors in the HPV-associated oropharynx. This suggests that these patients have a higher prevalence of HPV-negative cancer, which has been shown to have poorer survival(3-6), within classically defined HPVpositive subsites. This difference is potentially driven by an increased use of smoking and smokeless tobacco amongst males in the region. The implication of these findings is that because tobacco remains the primary cause of $\mathrm{HNSCC}(7)$ and Appalachia harbors the highest tobacco-use rates in the country(8), accurate tobacco histories of patients is an important predictor of clinical outcome, regardless of the anatomical location of the tumor. This work also suggests that the recent discussion of treatment de-intensification of patients with HPVassociated oropharyngeal cancers $(9,10)$ should be revisited, and that tobacco-exposure needs to be included in this discussion as a treatment qualifier. Importantly, p16 immunohistochemistry (IHC) is one of the criteria used when determining treatment for HNSCC patients(11). This diagnostic indicator is well supported(12,13), yet not a perfect indicator of HPV positivity due to other chromosomal alterations that can occur to skew these results to lead to false positives and false negatives(14). CDKN2A is often deleted in HNSCC(3) which would lead to a false negative result even if the patient is HPV-positive. Conversely, CCND1 would elicit the same loss of p16 negative-feedback loop by releasing more E2F transcription factor, which would also lead to an accumulation of p16 and could lead to be a false positive. These scenarios are detailed in Figure 2. 


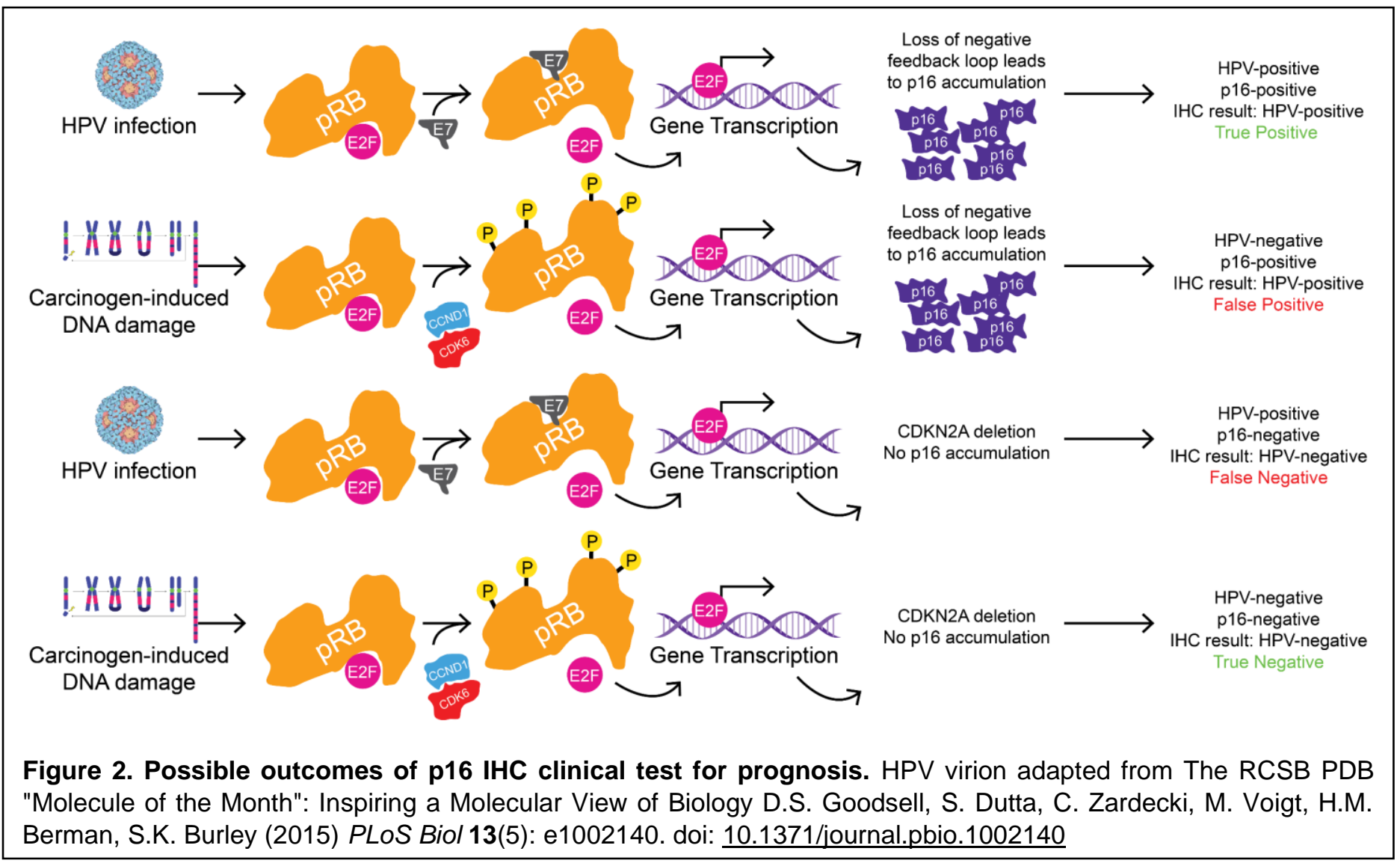

Since p16 IHC is currently paramount in determining the course of patient treatment, this could lead to disastrous results, even if this impacts a low number of patients. A better solution would be to perform CCND1, CDKN2A, and HPV PCR alongside p16 staining $(15,16)$. Unfortunately, with the advent of AJCC 8th edition putting more emphasis on HPV status by downstaging patients if they are HPV-positive by p16 staining, we are beginning to see evidence of potential undertreatment and worse outcomes of patients due to the under-staging and less aggressive treatment proposed in the AJCC 8th edition(9). Overall, these studies, coupled with the results of Study 1 , underscore the need for continued pursuit to fully understand molecular, behavioral, and geographical differences in the staging and treatment of HNSCC patients regardless of etiology. Future studies could track the effectiveness of smoking cessation campaigns in Appalachia, as well as effects of prophylactic HPV vaccination on HNSCC stage IV white male survival and how this effects patient survival over time. If the disparity does not normalize to national outcomes, it is imperative that continued government funding to the ARC, as well as continued education and smoking cessation campaigns will continue to be necessary for reducing HNSCC risk and improving survival in the Appalachian region. Such an approach can also be extrapolated to areas of disproportional smoking tobacco, smokeless tobacco(17), or betel-quid use(18). 
HNSCC is largely conceptualized by the field as being driven by genomic relief of tumor suppression(19). However, HNSCC also harbors many copy-number gains and amplifications, the most common of which is the $11 q 13$ amplicon. Chromosome $11 q 13$ amplification is found in nearly one third of all patients and is associated with lower survival $(3,20)$. Although it is believed 11 q13 amplification and other CNAs in HNSCC are associated with DNA damage arising from carcinogenic insult due to cigarette smoking and associated DNA damage $(7,21)$, the precise CNAs correlated with smoking have not been directly defined. The work outlined in Study 2 seeks to close this knowledge gap by correlating the copy number of 24,776 genes with the pack-year data of 509 patients from the well-annotated TCGA cohort to generate a smoking-associated copy number and expression landscape in HNSCC. This work resulted in identifying a smoking-associated expression signature (SAES) that mapped to four distinct chromosomal cytobands in known CNAs, including deletions of genes within 9p21.3 and 9p24.1 with the vast majority being amplifications of genes within $11 q 13.3$ and 11q13.4. All underexpressed and overexpressed genes within the SAES reduced patient overall survival and were correlated with an increased relative risk of death. Interestingly, there is a near-linear positive correlation between probability of amplification of 11q13.3-q13.4 and smoking pack year as well as between overexpression of 11q13.3-q13.4 SAES genes. The correlation of these CNAs coupled with this predictive aspect with increased smoking tobacco usage underscores the importance of collecting complete tobacco use histories during patient consultation and diagnosis. This history is simple to collect from the patient and may be useful to both researchers and clinicians to predict probability of smoking-associated DNA damage and expression SAES genes which could better guide early treatment. In addition to the known drivers of HNSCC within these CNAs, such as CCND1 and $\operatorname{CTTN}(22,23)$, we have also identified candidate drivers of HNSCC where overexpression reduces patient survival. A subset of these candidate drivers also correlates with lymph node metastasis, which is an important prognostic indicator of patient outcome $(24,25)$. These candidate genes include the mitochondrial ribosomal protein L21 (MRPL21), LAR protein tyrosine phosphatase-interacting protein alpha-1 (PPFIA1), fibroblast growth factor 19 (FGF19), two pore segment channel 2 (TPCN2), and E3 ubiquitin ligase RING finger protein 121 (RNF121). MRPL21 encodes a large mitochondria ribosomal subunit, where proper stoichiometry is critical for mitoribosome assembly(26). There is no defined role for MRPL21 in tumor progression, but its cellular function suggests a role in regulating tumor cell metabolism. PPFIA1 encodes a LAR phosphatase-interacting protein that localizes to and regulates the disassembly of focal adhesions(27). Liprin-alpha1 has a roles in regulating 
cell motility, invasion, and transmembrane signaling(28,29). FGF19 encodes a fibroblast growth factor that functions as a hormone in the regulation of bile acid synthesis, signaling through the fibroblast growth factor receptor 4 (FGFR4)(30) that is linked to tumor-promoting autocrine signaling(30). TPCN2 encodes a cationselective ion channel that releases calcium ions from lysosomal stores but does not have a defined role as a tumor promoter(31). RNF121 encodes an E3 polytopic membrane protein with five transmembrane regions thought to be part of the endoplasmic-reticulum-associated protein degradation response (ERAD) that downregulates several proteins in invertebrates(32). RNF121 does not have a well-defined role in tumor progression as it has been shown to be both a tumor promoter(33) and tumor suppressor(34), with a defined role in NF-KB activation(35). Future studies that expand our understanding of how $11 \mathrm{q} 13$ amplification and resultant gene overexpression of these poorly defined candidate genes contributes to poor patient outcome in smoking-associated HNSCC is further underscored by the increased resistance to chemotherapy and EGFR targeting in 11q13-amplified $\operatorname{HNSCC}(36-38)$. This highlights a persistent need for earlier and precise diagnosis for patients with SAES CNA and expression status to provide improved clinical management of tobacco-positive, HPV-negative HNSCC.

The 11q13 amplicon contains several genes that have been linked to increased tumor progression, including CCND1(19), CTTN(39), FADD(40), and ORAOV1(41). These genes drive tumor progression through gene product protein-protein interactions. However, other functions of the mRNA transcript itself, independent of protein function, could drive tumor progression. In Study 3, we explored the potential competing endogenous effects of the RNA of these genes on altering expression of other cellular RNAs and ultimately altering cellular expression of proteins involved in either tumor-suppressive or tumor-promoting roles. We observed that the overexpression of the CCND1 3'-UTR is associated with reduced patient survival and also contains 31 MREs for tumor-suppressive microRNAs. When correlating expression of CCND1 3'-UTR from RNAseq analysis and protein expression from reverse phase protein lysate microarray RPPA analysis, we identified several proteins of interest with increased differential expression encoded by transcripts containing MREs shared with the CCND1 3'-UTR. These proteins include TYMS, SERPINE1, CCNB1, DVL3, GATA3, E2F1, and ITGA2. All of these proteins have been linked to tumor progression, although their roles in HNSCC are only partially known $(42,43,52-61,44,62,63,45-51)$. The added effect of these overexpressed tumor promoters creates an additional layer of complexity to the oncogenic role of overexpressed cyclin D1. Elucidating the full impact of 
CCND1 mRNA overexpression, through 3'-UTR-mediated ceRNA sequestration or through cyclin D1 protein overexpression is imperative in understanding how to best stratify patients for treatment with CDK4/6 inhibitors that only impact CCND1 protein function. The amplification of 11q13 may not be enough in and of itself to justify stratification for CDK4/6 inhibitors. As shown in the PALOMA-1 clinical trial that stratified patients by CCND1 amplification and the results did not support that stratification by CCND1 amplification improves patient outcome $(64,65)$. Alternatively, there is evidence that stratification by CCND1 transcript expression is a poor predictor of response rates of other chemotherapy drugs(66). The results in this Study coupled with these stratification results suggest that CCND1 3'-UTR expression levels may be an important way to predict patient outcome from chemotherapeutic intervention. In the Addendum to Study 3, we created CCND1 expression vectors that overexpress different segments of the CCND1 transcript using in vitro assays to examine for the presence of a ceRNA-mediated effect on tumor progression. These constructs contained the ORF alone, full length $C C N D 1$, and a frameshift $C C N D 1$ mutant that does not produce cyclin D1 protein. Exogenous expression was confirmed using various in vitro assays including western blotting, RT-qPCR, and mRNA-FISH in both the non-11q13-amplified HNSCC cell line MDA-1586 and the 11q13-amplified HNSCC cell line UPCI:SCC040. Effect on cell cycle progression was also evaluated, with mixed results. For this reason, additional laboratory investigation is required to confirm that the suggested ceRNA effect is supported. Future experiments that examine effects of these proteins on tumor progression, such as transcriptome RNAseq or proteome mass spectrometry using the developed CCND1 constructs will be imperative for complete understanding of the dynamics at play. If the ceRNA effect is supported, there is potential to uncover new actionable targets that are deregulated due to the ceRNA effect. Additionally, confirming shared microRNA that interact with these target proteins will be an important first step to direct targeted anti-microRNA therapy approaches to mediate CCND1 3'-UTR-driven tumor progression. 


\section{Bibliography}

1. Wilson RJ, Ryerson AB, Singh SD, King JB. Cancer Incidence in Appalachia, 2004-2011. Cancer Epidemiol Biomarkers Prev [Internet]. 2016 Feb [cited 2016 Mar 8];25(February):200411. Available from: http://www.ncbi.nlm.nih.gov/pubmed/26819264

2. Bruce C. Casto, Smita Sharma, James L. Fisher, Thomas J. Knobloch, Amit Agrawal, Christopher M. Weghorst. Oral Cancer in Appalachia. J Health Care Poor Underserved. 2009;20(1):274-85.

3. Lawrence MS, Sougnez C, Lichtenstein L, Cibulskis K, Lander E, Gabriel SB, et al. Comprehensive genomic characterization of head and neck squamous cell carcinomas. Nature [Internet]. 2015;517(7536):576-82. Available from:

http://www.nature.com/doifinder/10.1038/nature14129

4. Keck MK, Zuo Z, Khattri A, Stricker TP, Brown CD, Imanguli M, et al. Integrative Analysis of Head and Neck Cancer Identifies Two Biologically Distinct HPV and Three Non-HPV Subtypes. Clin Cancer Res [Internet]. 2015;21(4):870-81. Available from: http://clincancerres.aacrjournals.org/cgi/doi/10.1158/1078-0432.CCR-14-2481

5. Maitra A, Biswas NK, Amin K, Kowtal P, Kumar S, Das S, et al. Mutational landscape of gingivo-buccal oral squamous cell carcinoma reveals new recurrently-mutated genes and molecular subgroups. Nat Commun [Internet]. 2013 Dec 2 [cited 2019 Apr 6];4(1):2873. Available from: http://www.nature.com/articles/ncomms3873

6. Stransky N, Egloff AM, Tward AD, Kostic AD, Sougnez C, Mckenna A, et al. The Mutational Landscape of Head Squamous Cell Carcinoma. Science (80- ). 2014;333(6046):1157-60.

7. Jethwa AR, Khariwala SS. Tobacco-related carcinogenesis in head and neck cancer. Cancer Metastasis Rev [Internet]. 2017 [cited 2020 Jul 14];36(3):411-23. Available from: http://www.ncbi.nlm.nih.gov/pubmed/28801840 
8. Behavioral Risk Factor Data: Tobacco Use (2011 to present) | Chronic Disease and Health Promotion Data \&amp; Indicators [Internet]. [cited 2019 Aug 4]. Available from: https://chronicdata.cdc.gov/Survey-Data/Behavioral-Risk-Factor-Data-Tobacco-Use-2011-topr/wsas-xwh5

9. Cheraghlou S, Yu PK, Otremba MD, Park HS, Bhatia A, Zogg CK, et al. Treatment deintensification in human papillomavirus-positive oropharynx cancer: Outcomes from the National Cancer Data Base. Cancer [Internet]. 2018 Feb 15 [cited 2020 Jun 29];124(4):717-26. Available from: http://doi.wiley.com/10.1002/cncr.31104

10. Oosthuizen JC, Kinsella JB. Is treatment de-escalation a reality in HPV related oropharyngeal cancer? Surgeon [Internet]. 2016;14(4):180-3. Available from:

http://dx.doi.org/10.1016/j.surge.2016.04.002

11. National Comprehensive Cancer Network. Head and Neck Cancers (Version 2.2020) [Internet]. 2020. Available from: https://www.nccn.org/professionals/physician_gls/pdf/head-and-neck.pdf

12. Weinberger PM, Yu Z, Haffty BG, Kowalski D, Harigopal M, Brandsma J, et al. Molecular classification identifies a subset of human papillomavirus--associated oropharyngeal cancers with favorable prognosis. J Clin Oncol [Internet]. 2006 Feb 10 [cited 2020 Jul 14];24(5):736-47. Available from: http://www.ncbi.nlm.nih.gov/pubmed/16401683

13. Cantley RL, Gabrielli E, Montebelli F, Cimbaluk D, Gattuso P, Petruzzelli G. Ancillary studies in determining human papillomavirus status of squamous cell carcinoma of the oropharynx: a review. Patholog Res Int [Internet]. 2011 [cited 2020 Jul 14];2011:138469. Available from: http://www.ncbi.nlm.nih.gov/pubmed/21772959

14. Rietbergen MM, Snijders PJF, Beekzada D, Braakhuis BJM, Brink A, Heideman DAM, et al. Molecular characterization of p16-immunopositive but HPV DNA-negative oropharyngeal carcinomas. Int J Cancer. 2014;134(10):2366-72. 
15. Huang X, Godfrey TE, Gooding WE, McCarty KS, Gollin SM. Comprehensive genome and transcriptome analysis of the $11 \mathrm{q} 13$ amplicon in human oral cancer and synteny to the 7F5 amplicon in murine oral carcinoma. Genes Chromosom Cancer. 2006;45(11):1058-69.

16. Arezoo Mohajeri, 1 Johnbosco Tayebwa, 1 Anna Collin, 1 Jenny Nilsson, 1 Linda Magnusson, 1 Fredrik Vult von Steyern 2 Otte Brosj€, o, 3 Henryk A. Domanski, 4 Olle Larsson, 5 Raf Sciot, 6 Maria Debiec-Rychter, 7 Jason L. Hornick, 8 Nils Mandahl, 1 Karolin H. Nord 1 and Fredrik Mertens. Comprehensive Genetic Analysis Identifies a Pathognomonic NAB2/STAT6 Fusion Gene, Nonrandom Secondary Genomic Imbalances, and a Characteristic Gene Expression Profile in Solitary Fibrous Tumor. Genes Chromosomes Cancer. 2013;52(1):873-86.

17. Critchley JA, Unal B. Health effects associated with smokeless tobacco: A systematic review. Thorax. 2003;58(5):435-43.

18. Tangjaturonrasme N, Vatanasapt $\mathrm{P}$, Bychkov A. Epidemiology of head and neck cancer in Thailand. Asia Pac J Clin Oncol. 2018;14(1):16-22.

19. Birkeland AC, Ludwig ML, Spector ME, Brenner JC. The potential for tumor suppressor gene therapy in head and neck cancer. Discov Med [Internet]. 2016;21(113):41-7. Available from: http://www.ncbi.nlm.nih.gov/pubmed/26896601\%5Cnhttp://www.pubmedcentral.nih.gov/articler ender.fcgi?artid=PMC4772772

20. Scott D. Meredith, Paul A. Levine, James A. Burns, Michael J. Gaffey, James C. Boyd, Lawrence M. Weiss, Nicholette L. Erickson MEW, M L, Levine PA, Burns JA, Gaffey MJ, Boyd JC, et al. Chromosome 11q13 Amplification in Head and Neck Squamous Cell Carcinoma. Association with poor prognosis. Arch Otolaryngol Head Neck Surg. 1995;121(1):31-54.

21. Hayes DN, Van Waes C, Seiwert TY. Genetic landscape of human papillomavirus-associated head and neck cancer and comparison to tobacco-related tumors. J Clin Oncol [Internet]. 2015 Oct 10 [cited 2020 Jun 19];33(29):3227-34. Available from: 
http://www.ncbi.nlm.nih.gov/pubmed/26351353

22. Ramos-García P, Ruiz-Ávila I, Gil-Montoya JA, Ayén Á, González-Ruiz L, Navarro-Triviño FJ, et al. Relevance of chromosomal band 11q13 in oral carcinogenesis: An update of current knowledge. Oral Oncol. 2017;72(2017):7-16.

23. Schuuring $\mathrm{E}$. The involvement of the chromosome 11q13 region in human malignancies: cyclin D1 and EMS1 are two new candidate oncogenes- a review *. 1995;159:83-96.

24. Goldson TM, Han Y, Knight KB, Weiss HL, Resto VA. Clinicopathological predictors of lymphatic metastasis in HNSCC: implications for molecular mechanisms of metastatic disease. J Exp Ther Oncol [Internet]. 2010;8(3):211-21. Available from: http://www.pubmedcentral.nih.gov/articlerender.fcgi?artid=2929983\&tool=pmcentrez\&rendertyp $\mathrm{e}=\mathrm{abstract}$

25. Ferlito A, Rinaldo A, Devaney KO, MacLennan K, Myers JN, Petruzzelli GJ, et al. Prognostic significance of microscopic and macroscopic extracapsular spread from metastatic tumor in the cervical lymph nodes. Oral Oncol. 2002;38(8):747-51.

26. Kim HJ, Maiti P, Barrientos A. Mitochondrial ribosomes in cancer [Internet]. Vol. 47, Seminars in Cancer Biology. Semin Cancer Biol; 2017 [cited 2020 Jul 12]. p. 67-81. Available from: http://www.ncbi.nlm.nih.gov/pubmed/28445780

27. Serra-Pagès C, Medley QG, Tang M, Hart A, Streuli M. Liprins, a family of LAR transmembrane protein-tyrosine phosphatase- interacting proteins. J Biol Chem. 1998;273(25):15611-20.

28. Astro V, Asperti C, Cangi G, Doglioni C, de Curtis I. Liprin-a1 regulates breast cancer cell invasion by affecting cell motility, invadopodia and extracellular matrix degradation. Oncogene [Internet]. 2011;30(15):1841-9. Available from:

http://www.nature.com/doifinder/10.1038/onc.2010.562 
29. Pehkonen H, Lento M, Von Nandelstadh P, Filippou A, Grénman R, Lehti K, et al. Liprin-a1 modulates cancer cell signaling by transmembrane protein CD82 in adhesive membrane domains linked to cytoskeleton. Cell Commun Signal. 2018;16(1):1-14.

30. Gao L, Lang L, Zhao X, Shay C, Shull AY, Teng Y. FGF19 amplification reveals an oncogenic dependency upon autocrine FGF19/FGFR4 signaling in head and neck squamous cell carcinoma. Oncogene [Internet]. 2019 [cited 2020 Jun 19];38(13):2394-404. Available from: https://pubmed.ncbi.nlm.nih.gov/30518874/

31. Zong X, Schieder M, Cuny H, Fenske S, Gruner C, Rötzer K, et al. The two-pore channel TPCN2 mediates NAADP-dependent $\mathrm{Ca}(2+)$-release from lysosomal stores. Pflugers Arch [Internet]. 2009 Sep [cited 2020 Jul 14];458(5):891-9. Available from: http://www.ncbi.nlm.nih.gov/pubmed/19557428

32. Darom A, Bening-Abu-Shach U, Broday L. RNF-121 is an endoplasmic reticulum-membrane E3 ubiquitin ligase involved in the regulation of $\beta$-integrin. Mol Biol Cell [Internet]. 2010 Jun 1 [cited 2020 Jul 12];21(11):1788-98. Available from: http://www.ncbi.nlm.nih.gov/pubmed/20357004

33. Choi Y, Kwon CH, Lee SJ, Park J, Shin JY, Park DY. Integrative analysis of oncogenic fusion genes and their functional impact in colorectal cancer. Br J Cancer [Internet]. 2018 [cited 2020 Jul 12];119(2):230-40. Available from: http://www.ncbi.nlm.nih.gov/pubmed/29955133

34. Xiang P, Sun Y, Liu Y, Shu Q, Zhu Y. Really interesting new gene finger protein 121 is a tumor suppressor of renal cell carcinoma. Gene [Internet]. 2018 Nov 15 [cited 2020 Jul 12];676:3228. Available from: http://www.ncbi.nlm.nih.gov/pubmed/30149063

35. Zemirli N, Pourcelot M, Dogan N, Vazquez A, Arnoult D. The E3 ubiquitin ligase RNF121 is a positive regulator of NF-KB activation. Cell Commun Signal [Internet]. 2014 Nov 12 [cited 2020 Jul 12];12(1):72. Available from: http://www.ncbi.nlm.nih.gov/pubmed/25388546

36. Timpson P, Wilson AS, Lehrbach GM, Sutherland RL, Musgrove EA, Daly RJ. Aberrant 
expression of cortactin in head and neck squamous cell carcinoma cells is associated with enhanced cell proliferation and resistance to the epidermal growth factor receptor inhibitor gefitinib. Cancer Res. 2007;67(19):9304-14.

37. Zhong L-P, Zhu D-W, William WN, Liu Y, Ma J, Yang C-Z, et al. Elevated Cyclin D1 Expression Is Predictive for a Benefit from TPF Induction Chemotherapy in Oral Squamous Cell Carcinoma Patients with Advanced Nodal Disease. Mol Cancer Ther. 2013;12(6):1112-21.

38. Zhang P, Zhang Z, Zhou X, Qiu W, Chen F, Chen W. Identification of genes associated with cisplatin resistance in human oral squamous cell carcinoma cell line. BMC Cancer. 2006;6:111.

39. Rothschild BL, Shim AH, Ammer AG, Kelley LC, Irby KB, Head JA, et al. Cortactin overexpression regulates actin-related protein $2 / 3$ complex activity, motility, and invasion in carcinomas with chromosome 11q13 amplification. Cancer Res [Internet]. 2006 Aug 15 [cited 2020 Jul 13];66(16):8017-25. Available from: http://www.ncbi.nlm.nih.gov/pubmed/16912177

40. Pattje WJ, Melchers LJ, Slagter-Menkema L, Mastik MF, Schrijvers ML, Gibcus JH, et al. FADD expression is associated with regional and distant metastasis in squamous cell carcinoma of the head and neck. Histopathology. 2013;63(2):263-70.

41. Zhai C, Li Y, Mascarenhas C, Lin Q, Li K, Vyrides I, et al. The function of ORAOV1/LTO1, a gene that is overexpressed frequently in cancer: Essential roles in the function and biogenesis of the ribosome. Oncogene [Internet]. 2014 Jan 14 [cited 2020 Jul 2];33(4):484-94. Available from: http://www.nature.com/articles/onc2012604

42. Peters GJ, Backus HHJ, Freemantle S, Van Triest B, Codacci-Pisanelli G, Van der Wilt CL, et al. Induction of thymidylate synthase as a 5-fluorouracil resistance mechanism [Internet]. Vol. 1587, Biochimica et Biophysica Acta - Molecular Basis of Disease. Biochim Biophys Acta; 2002 [cited 2020 Jul 13]. p. 194-205. Available from: http://www.ncbi.nlm.nih.gov/pubmed/12084461 
43. Hardy LW, Finer-Moore JS, Montfort WR, Jones MO, Santi D V, Stroud RM. Atomic structure of thymidylate synthase: Target for rational drug design. Science (80- ) [Internet]. 1987 Jan 23 [cited 2020 Jul 13];235(4787):448-55. Available from:

http://www.ncbi.nlm.nih.gov/pubmed/3099389

44. Yang JD, Ma L, Zhu Z. SERPINE1 as a cancer-promoting gene in gastric adenocarcinoma: facilitates tumour cell proliferation, migration, and invasion by regulating EMT. J Chemother [Internet]. 2019 [cited 2020 Jul 13];31(7-8):408-18. Available from:

http://www.ncbi.nlm.nih.gov/pubmed/31724495

45. Vachher M, Arora K, Burman A, Kumar B. NAMPT, GRN, and SERPINE1 signature as predictor of disease progression and survival in gliomas. J Cell Biochem [Internet]. 2020 [cited $2020 \mathrm{Jul}$ 13];121(4):3010-23. Available from: https://pubmed.ncbi.nlm.nih.gov/31710121/

46. Wang D, Yang LY, Liu Z, Yu J, Zhang MJ, Zhang Y, et al. PAl-1 overexpression promotes invasion and migration of esophageal squamous carcinoma cells. Yi chuan = Hered [Internet]. 2020 Mar 20 [cited 2020 Jul 13];42(3):287-95. Available from:

http://www.ncbi.nlm.nih.gov/pubmed/32217514

47. Kubala MH, DeClerck YA. The plasminogen activator inhibitor-1 paradox in cancer: a mechanistic understanding [Internet]. Vol. 38, Cancer and Metastasis Reviews. Cancer Metastasis Rev; 2019 [cited 2020 Jul 13]. p. 483-92. Available from:

http://www.ncbi.nlm.nih.gov/pubmed/31734763

48. Yuan J, Krämer A, Matthess Y, Yan R, Spänkuch B, Gätje R, et al. Stable gene silencing of cyclin B1 in tumor cells increases susceptibility to taxol and leads to growth arrest in vivo. Oncogene [Internet]. 2006 Mar 16 [cited 2020 Jul 13];25(12):1753-62. Available from: http://www.ncbi.nlm.nih.gov/pubmed/16278675

49. Kushner J, Bradley G, Young B, Jordan RCK. Aberrant expression of cyclin A and cyclin B1 
proteins in oral carcinoma. J Oral Pathol Med [Internet]. 2007 Feb [cited 2020 Jul 13];28(2):7781. Available from: http://www.ncbi.nlm.nih.gov/pubmed/9950254

50. Kawamoto H, Koizumi H, Uchikoshi T. Expression of the G2-M checkpoint regulators cyclin B1 and cdc2 in nonmalignant and malignant human breast lesions: Immunocytochemical and quantitative image analyses. Am J Pathol [Internet]. 1997 Jan [cited 2020 Jul 13];150(1):15-23. Available from: http://www.ncbi.nlm.nih.gov/pubmed/9006317

51. Wang A, Yoshimi N, Ino N, Tanaka T, Mori H. Overexpression of cyclin B1 in human colorectal cancers. J Cancer Res Clin Oncol [Internet]. 1997 [cited 2020 Jul 13];123(2):124-7. Available from: http://www.ncbi.nlm.nih.gov/pubmed/9030252

52. Mashal RD, Lester S, Corless C, Richie JP, Chandra R, Propert KJ, et al. Expression of cell cycle-regulated proteins in prostate cancer. Cancer Res [Internet]. 1996 Sep 15 [cited 2020 Jul 13];56(18):4159-63. Available from: http://www.ncbi.nlm.nih.gov/pubmed/8797586

53. Pizzuti A, Amati F, Calabrese G, Mari A, Colosimo A, Silani V, et al. cDNA characterization and chromosomal mapping of two human homologues of the Drosophila dishevelled polarity gene. Hum Mol Genet [Internet]. 1996 Jul [cited 2020 Jul 13];5(7):953-8. Available from: http://www.ncbi.nlm.nih.gov/pubmed/8817329

54. Li K, Zhang J, Tian Y, He Y, Xu X, Pan W, et al. The Wnt/ß-catenin/VASP positive feedback loop drives cell proliferation and migration in breast cancer. Oncogene [Internet]. 2020 Mar [cited $2020 \mathrm{Jul} 13$ ];39(11):2258-74. Available from:

http://www.ncbi.nlm.nih.gov/pubmed/31831834

55. Karin-Kujundzic V, Kardum V, Sola IM, Paic F, Skrtic A, Skenderi F, et al. Dishevelled family proteins in serous ovarian carcinomas: a clinicopathologic and molecular study. APMIS [Internet]. 2020 Mar [cited 2020 Jul 13];128(3):201-10. Available from: http://www.ncbi.nlm.nih.gov/pubmed/31755579 
56. Janssen JWG, Schulz AS, Steenvoorden ACM, Schmidberger M, Strehl Ambros SPF, Bartram CR. A novel putative tyrosine kinase receptor with oncogenic potential. Oncogene [Internet]. 1991 Nov 1 [cited 2020 Jul 13];6(11):2113-20. Available from: http://www.ncbi.nlm.nih.gov/pubmed/1834974

57. Wang L, Gao Y, Zhao X, Guo C, Wang X, Yang Y, et al. HOXD3 was negatively regulated by YY1 recruiting HDAC1 to suppress progression of hepatocellular carcinoma cells via ITGA2 pathway. Cell Prolif [Internet]. 2020 Jun 17 [cited 2020 Jul 13];e12835. Available from: http://www.ncbi.nlm.nih.gov/pubmed/32557953

58. Ma L, Sun Y, Li D, Li H, Jin X, Ren D. Overexpressed ITGA2 contributes to paclitaxel resistance by ovarian cancer cells through the activation of the AKT/FoxO1 pathway. Aging (Albany NY) [Internet]. 2020 [cited 2020 Jul 13];12(6):5336-51. Available from: http://www.ncbi.nlm.nih.gov/pubmed/32202508

59. Ren D, Zhao J, Sun Y, Li D, Meng Z, Wang B, et al. Overexpressed ITGA2 promotes malignant tumor aggression by up-regulating PD-L1 expression through the activation of the STAT3 signaling pathway. J Exp Clin Cancer Res [Internet]. 2019 Dec 9 [cited 2020 Jul 13];38(1):485. Available from: http://www.ncbi.nlm.nih.gov/pubmed/31818309

60. Gerger A, Hofmann G, Langsenlehner U, Renner W, Weitzer W, Wehrschütz M, et al. Integrin alpha-2 and beta-3 gene polymorphisms and colorectal cancer risk. Int J Colorectal Dis [Internet]. 2009 Feb [cited 2020 Jul 13];24(2):159-63. Available from:

http://www.ncbi.nlm.nih.gov/pubmed/18836731

61. Langsenlehner U, Renner W, Yazdani-Biuki B, Eder T, Wascher TC, Paulweber B, et al. Integrin alpha-2 and beta-3 gene polymorphisms and breast cancer risk. Breast Cancer Res Treat [Internet]. 2006 May [cited 2020 Jul 13];97(1):67-72. Available from: http://www.ncbi.nlm.nih.gov/pubmed/16317580 
62. Shan L, Li X, Liu L, Ding X, Wang Q, Zheng Y, et al. GATA3 cooperates with PARP1 to regulate CCND1 transcription through modulating histone $\mathrm{H} 1$ incorporation. Oncogene [Internet]. 2014 Jun 12 [cited 2020 Jul 15];33(24):3205-16. Available from:

http://www.ncbi.nlm.nih.gov/pubmed/23851505

63. Yang $\mathrm{XH}$, Sladek TL. Overexpression of the E2F-1 transcription factor gene mediates cell transformation. Gene Expr [Internet]. 1995 [cited 2020 Jul 15];4(4-5):195-204. Available from: http://www.ncbi.nlm.nih.gov/pubmed/7787412

64. Knudsen ES, Witkiewicz AK. The Strange Case of CDK4/6 Inhibitors: Mechanisms, Resistance, and Combination Strategies. Trends in Cancer [Internet]. 2017 Jan 1 [cited 2020 Jul 14];3(1):39-55. Available from:

https://www.sciencedirect.com/science/article/abs/pii/S2405803316301844

65. Finn RS, Crown JP, Lang I, Boer K, Bondarenko IM, Kulyk SO, et al. The cyclin-dependent kinase $4 / 6$ inhibitor palbociclib in combination with letrozole versus letrozole alone as first-line treatment of oestrogen receptor-positive, HER2-negative, advanced breast cancer (PALOMA1/TRIO-18): a randomised phase 2 study. Lancet Oncol [Internet]. 2015 Jan [cited 2020 Jul 14];16(1):25-35. Available from: http://www.ncbi.nlm.nih.gov/pubmed/25524798

66. Tanioka M, Sakai K, Sudo T, Sakuma T, Kajimoto K, Hirokaga K, et al. Transcriptional CCND1 expression as a predictor of poor response to neoadjuvant chemotherapy with trastuzumab in HER2-positive/ER-positive breast cancer. Breast Cancer Res Treat [Internet]. 2014 Oct [cited 2020 Jul 14];147(3):513-25. Available from: http://www.ncbi.nlm.nih.gov/pubmed/25200445 


\section{Cortactin Phosphorylation by Casein Kinase 2 Regulates Actin-Related Protein 2/3 Complex Activity, Invadopodia Function, and Tumor Cell Invasion}

Steven M. Markwell', Amanda G. Ammer', Erik T. Interval'2, Jessica L. Allen', Brenen W. Papenberg', River A. Hames', Johnathan E. Castaño ${ }^{2}$, Dorothy A. Schafer ${ }^{3}$, and Scott A. Weed ${ }^{1}$

\section{Abstract}

Malregulation of the actin cytoskeleton enhances tumor cell motility and invasion. The actin-binding protein cortactin facilitates branched actin network formation through activation of the actin-related protein (Arp) 2/3 complex. Increased cortactin expression due to gene amplification is observed in head and neck squamous cell carcinoma (HNSCC) and other cancers, corresponding with elevated tumor progression and poor patient outcome. Arp2/3 complex activation is responsible for driving increased migration and extracellular matrix (ECM) degradation by governing invadopodia formation and activity. Although cortactin-mediated activation of Arp $2 / 3$ complex and invadopodia regulation has been well established, signaling pathways responsible for governing cortactin binding to Arp2/3 are unknown and potentially present a new avenue for anti-invasive therapeutic targeting. Here we identify casein kinase (CK) $2 \alpha$ phosphorylation of cortactin as a negative regulator of $\operatorname{Arp} 2 / 3$ binding. CK2 $\alpha$ directly phosphorylates cortactin at a conserved threonine (T24) adjacent to the canonical Arp2/3 binding motif. Phosphorylation of cortactin T 24 by CK $2 \alpha$ impairs the ability of cortactin to bind Arp2/3 and activate actin nucleation. Decreased invadopodia activity is observed in HNSCC cells with expression of CK2 $\alpha$ phosphorylationnull cortactin mutants, shRNA-mediated CK2 $\alpha$ knockdown, and with the CK2 $\alpha$ inhibitor Silmitasertib. Silmitasertib inhibits HNSCC collective invasion in tumor spheroids and orthotopic tongue tumors in mice. Collectively these data suggest that CK2 $\alpha$-mediated cortactin

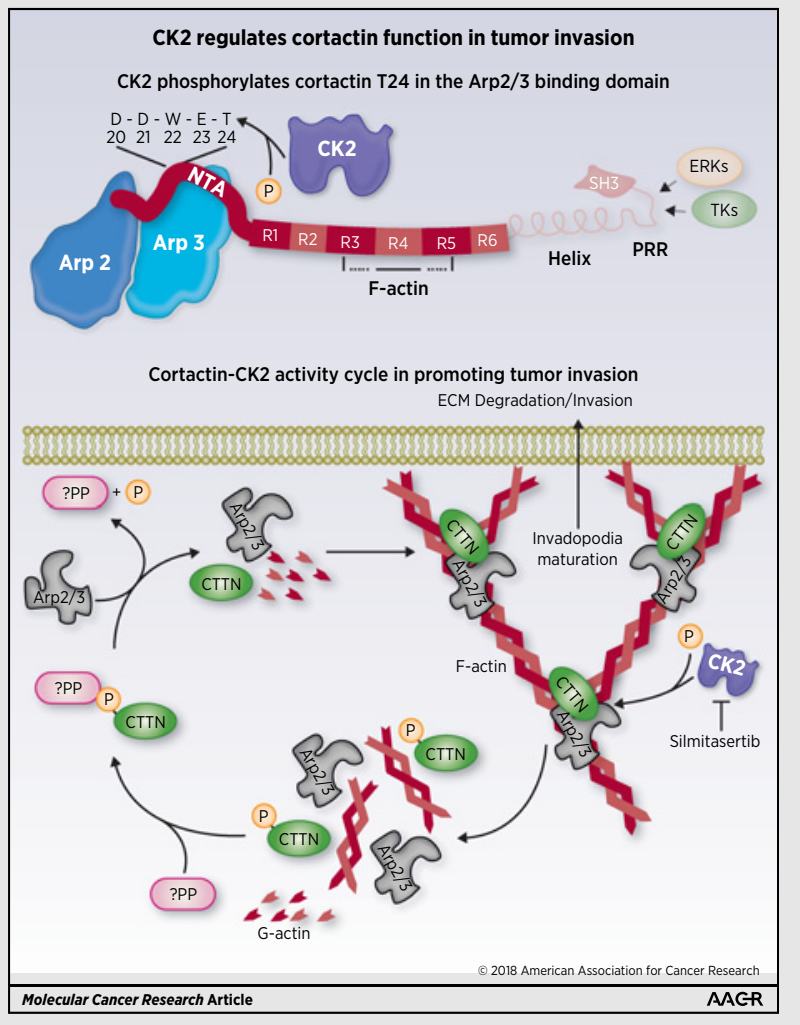
phosphorylation at T24 is critical in regulating cortactin binding to Arp2/3 complex and pro-invasive activity, identifying a potential targetable mechanism for impairing HNSCC invasion.

Implications: This study identifies a new signaling pathway that contributes to enhancing cancer cell invasion. Visual Overview: http://mcr.aacrjournals.org/content/molcanres/17/4/987/F1.large.jpg.

${ }^{1}$ Program in Cancer Cell Biology, Department of Biochemistry, West Virginia University, Morgantown, West Virginia. ${ }^{2}$ Department of Otolaryngology, Head and Neck Surgery, West Virginia University, Morgantown, West Virginia. ${ }^{3}$ Department of Biology, University of Virginia, Charlottesville, Virginia.

Note: Supplementary data for this article are available at Molecular Cancer Research Online (http://mcr.aacrjournals.org/).
Corresponding Author: Scott A. Weed, West Virginia University School of Medicine, 1 Medical Center Dr, Morgantown, WV 26506-9300. Phone: 304293-3016, Fax: 304-293-4667, E-mail: scweed@hsc.wvu.edu.

doi: 10.1158/1541-7786.MCR-18-0391

(C)2019 American Association for Cancer Research. 


\section{Introduction}

Cell invasion from the primary tumor is responsible for initiating the metastatic cascade and increasing cancer lethality $(1,2)$. Invasion is initiated in part through the action of invadopodia, actin-based membrane protrusions produced by tumor cells that mediate dissemination by degrading restrictive extracellular matrix (ECM) proteins through enzymatic matrix metalloproteinase (MMP) activity (3). Invadopodia contain a central filamentous (F-) actin core surrounded by an integrin-based adhesion ring complex that anchors the structure to allow focal matrix degradation and tumor cell protrusion through the basement membrane (4). Cortactin and actin-related protein (Arp) 2/3 complex are essential protein components involved in invadopodia precursor core formation required for subsequent MMP recruitment and membrane protrusion $(5,6)$. Cortactin overexpression is common in several cancer types including head and neck squamous cell carcinoma (HNSCC), resulting in enhanced motility, invasion, and invadopodia activity $(7,8)$. Cortactin binding to Arp2/3 complex activates Arp2/3 actin nucleation activity, enhancing cellular actin polymerization to form branched F-actin networks $(6,8-10)$. Cortactin also directly binds F-actin and bundles newly-formed filaments, providing an overall stabilizing effect on the Arp2/3-F-actin network required for invadopodia formation $(5,6,11)$. Previous work has shown that a DDW motif within the cortactin N-terminal acidic (NTA) domain is central in mediating Arp2/3 activation and branched actin network formation $(7,12-14)$. This region is similar to the Arp2/3 binding motif found in the acidic region of the Verprolin, Central, Acidic (VCA) domain of the WiskottAldrich Syndrome protein (WASp)-family of Arp2/3 nucleation promotion factors (NPF). Although the cortactin DDW motif is well established as the region responsible for Arp2/3 binding, posttranslational or other modifications of amino acids in the NTA region that regulate binding have not been reported. Tyrosine and serine phosphorylation of cortactin residues in the carboxyl-terminal region are essential for invadopodia formation, cellular invasion, and tumor metastasis through multiple mechanisms ultimately involving activation of WASp NPFs $(6,7,15-18)$. In addition, comprehensive phosphorylation site mapping by mass spectroscopy has identified NTA phosphorylation sites in close proximity to the DDW motif (19). This raises the possibility that phosphorylation of one or more of these residues may serve to govern Arp2/3 binding and invadopodia function in invasive cancer.

Casein kinase (CK) 2 is a ubiquitously expressed, constitutively active serine/threonine kinase consisting of two catalytic subunits ( $\alpha$ or $\alpha^{\prime}$ ) and two $\beta$ regulatory subunits (20). Increased CK2 expression correlates with cell-cycle progression, apoptosis resistance, and tumor cell motility in various cancers (20). Overexpressed CK2 enhances HNSCC tumor cell motility (20). CK2 phosphorylates the cortactin homologue HS1 at an unidentified site(s) in the NTA region (21), as well as residues near the DDW region in the NPFs neuronal (N)-WASp and WAS protein family member 2 (WASF2; WAVE2, WASp family Verprolin-homologous protein 2; refs. 22-25). Here we show that CK2 phosphorylation of threonine (T) 24 in the cortactin NTA impairs binding to and activation of Arp2/3 complex. Cortactin T2 4 and CK2 are required for efficient invadopodia formation and ECM degradation activity in HNSCC cell lines. Treatment of established and primary HNSCC cells with the selective CK2 inhibitor Silmitasertib impairs invadopodia function and regional HNSCC invasion. These results identify a new mechanism of invadopodia regulation that can be targeted to impair HNSCC invasion.

\section{Materials and Methods}

Cell culture, lentiviral infection and transfection, siRNA

HNSCC cell lines OSC19 and UMSCC1 were acquired and maintained as described (26). MDA1586 cells were obtained in March 2014 from Barbara Frederick (University of Colorado, Denver, CO). All HNSCC lines were authenticated by STR profiling at the University of Arizona Genetics Core in June 2017. PCR-based mycoplasma testing (13100-01; Southern Biotech) was conducted on OSC19 and UMSCC1 lines in March 2015 and were free of contamination. The MDA1586 line was not tested for mycoplasma. HEK293T/17 cells were obtained in April 2013 from Robert Wysolmerski (West Virginia University, Morgantown, WV). NIH3T3 cells were obtained in June 2017 from Ivan Martinez (West Virginia University, Morgantown, WV) and maintained for $\leq 10$ passages. These lines were not authenticated or tested for mycoplasma. Cells were propagated in DMEM supplemented with $10 \%$ FBS and $1 \%$ penicillin-streptomycin for $<6$ months. OSC19 and UMSCC1 cells stably infected with pLKO.1-puro cortactin shRNA or CK2 $\alpha$ shRNA were generated by clonal puromycin selection following standard methods (CSNK2A1: TRCN0000380839, TRCN0000027627; CTTN: TRCN0000040275).

UMSCC1 cells stably infected with pLU-Luc2 expressing luciferase were generated following standard methods. Murine cortactin rescue OSC19 and UMSCC1 cells containing cortactin shRNA stably infected with pLenti CMV Hygro cortactin constructs were generated by subsequent clonal hygromycin selection. Complete cortactin knockdown in OSC19 and UMSCC1 cells was achieved by transfection of cortactin-targeting siRNA (ON-TARGETplus SMARTpool L-010508-00-0020; Dharmacon) using a Nucleofector I (Amaxa Biosystems).

\section{Western blotting, antibodies, and immunoprecipitation}

Western blotting was conducted as described (27) and visualized with autoradiography film (E3012; Denville Scientific) or captured by an Amersham Imager 600 (GE Healthcare BioSciences). Antibodies used were: anti-cortactin clone $4 \mathrm{~F} 11$ ( $1 \mu \mathrm{g} / \mathrm{mL}$; ref. 26), anti-pS473 AKT (\#4060, 1:1,000; Cell Signaling Technology), anti-panAKT (\#2920, 1:1,000; Cell Signaling Technology), anti- $\beta$-actin (\#8457, 1:1,000; Cell Signaling Technology), anti-CK2 $\alpha$ (\#2656, 1:500; Cell Signaling Technology), anti-DYKDDDK (FLAG) clone 2EL-1B11 (MAB3118, 1:500; Millipore), and anti-Arp3 (\#07-272, 1:500; EMD Millipore). Immunoprecipitation was conducted from cells lysed in 50 mmol/L Tris Buffer pH 8.0 with $10 \mathrm{mmol} / \mathrm{L}$ EDTA and $1 \%$ NP-40 (28). Clarified lysates $(1 \mathrm{mg})$ were incubated with $50 \mu \mathrm{L}$ of FLAG M2 affinity resin (A2220; Sigma-Aldrich) for 2 hours at $4^{\circ} \mathrm{C}$. Immune complexes were collected by centrifugation, washed twice with Tris buffer, separated by SDS-PAGE, and Western blotted with antibodies as described above.

Gelatin degradation assay, invadopodia characterization, and microscopy

Cells were plated on Oregon Green 488-conjugated gelatin (G13186; Invitrogen) coated coverslips (29). In cases of inhibitor treatment, cells were allowed to attach for 1 hour, then incubated 
for 12 or 24 hours with Silmitasertib (S2248; Selleckchem) as indicated. Cells were rinsed in PBS, fixed with $10 \%$ buffered formalin (SF100-4; Fisher Scientific), and labeled as described (29). Antibodies used were 4F11 (1:500) or anti-FLAG (1:500). Primary antibodies were visualized using Alexa Fluor 647 conjugated goat anti-mouse secondary antibody (A21235, 1:500; Invitrogen). F-actin was visualized with rhodamine-conjugated phalloidin (R415, 1:1,000; Invitrogen). Coverslips were mounted using ProLong Gold antifade with DAPI (P36935; Invitrogen). Images for quantifying gelatin degradation and knockdown/ rescue expression were acquired with a Zeiss Axio Imager Z2 epifluorescent microscope equipped with an AxioCam MRm CCD camera and AxioVision software using LD Plan-Neofluar 40X/0.6 Corr and Plan-Apochromat 63X/1.4 oil objectives (Carl Zeiss Microscopy). Acquisition parameters were held constant within comparison groups. Confocal images were acquired using a Zeiss Axio Imager Z1 LSM510 confocal microscope with EC Plan-Neofluar 40X/1.30 and Plan-Apochromat 63X/1.4 oil objectives and Zen2009 software (Carl Zeiss Microcopy). All representative images were level adjusted to enhance contrast and brightness as needed and resized using Photoshop CC 2018 (Adobe Systems). Gelatin images were corrected for uneven illumination via bandpass filtering using ImageJ software (NIH). Degradation and invadopodia formation was quantified as described previously (29), with $n \geq 70$ lentiviral infected or $\geq 100$ inhibitortreated cells evaluated for each condition. FLAG-stained control images were thresholded against nonspecific staining using ImageJ software. Cells above threshold values were considered positive for rescue construct expression and used for quantitation. For therapeutic treatments and RNAi stable cell lines, degradation and cell areas were determined by Image J (NIH) on an individual cell basis. Data represent the mean values normalized to control degradation area per cell area from at least three independent experiments. Invadopodia precursors were determined by colocalization of actin and cortactin at sites lacking gelatin degradation. Active invadopodia were determined by colocalization of actin, cortactin, and gelatin degradation. Data represent the mean from at least three independent experiments. Phase contrast images were acquired using a Zeiss Axiovert 200M microscope equipped with an AxioCamMR CCD camera using a Plan-Neofluar $10 \times / 0.30$ objective and AxioVision software (Carl Zeiss Microscopy).

\section{CK2 $\alpha$ kinase assay}

In vitro kinase assays were performed as described (30). Briefly, $0.25,0.5$, or $1 \mu \mathrm{g}$ of purified GST-wild-type (WT) or T24A cortactin NTA fusion proteins were incubated with $8 \mathrm{ng}$ CK2 $\alpha$ (\#14-445; Millipore) and $10 \mu \mathrm{Ci}^{32} \mathrm{P} \gamma$-ATP (\#NEG002A500UC; PerkinElmer) at $30^{\circ} \mathrm{C}$ for 10 minutes. Reactions were terminated with hot SDS sample loading buffer. Proteins were visualized by autoradiography. Purified N-WASp GST-VCA $(0.5 \mu \mathrm{g})$ and GST $(1 \mu \mathrm{g})$ were used as respective positive and negative controls.

\section{In vitro cortactin phosphorylation binding assay}

Purified WT or T24A cortactin proteins $(2.5 \mu \mathrm{g})$ were bound to 4F11-conjugated protein G magnetic beads (\#10003D; Life Technologies). Immune complexes were incubated in the presence or absence of activated CK2 $\alpha$ (75 ng; \#V4482; Promega) and ATP (500 nmol, \#BP413-25; Fisher Scientific) at $30^{\circ} \mathrm{C}$ for 15 minutes. Reactions were washed twice with $10 \mathrm{mmol} / \mathrm{L}$ Tris $\mathrm{pH} 7.4$, $150 \mathrm{mmol} / \mathrm{L} \mathrm{NaCl}, 0.5 \mathrm{mmol} / \mathrm{L}$ EDTA. Complexes were washed once with $10 \mathrm{mmol} / \mathrm{L}$ Tris $\mathrm{pH} 7.4,10 \mathrm{mmol} / \mathrm{L}$ EDTA, and incubated with 50 ng Arp2/3 complex (\#RP01-A; Cytoskeleton) at $4^{\circ} \mathrm{C}$ for 30 minutes. Following incubation, binding complexes were washed once with $10 \mathrm{mmol} / \mathrm{L}$ Tris Buffer $\mathrm{pH} 7.4$ with $25 \mathrm{mmol} / \mathrm{L} \mathrm{NaCl}, 10 \mathrm{mmol} / \mathrm{L}$ EDTA, $1 \% \mathrm{NP}-40$, then boiled and Western blotted with antibodies against cortactin and Arp3.

\section{Actin polymerization assay}

Actin polymerization experiments were conducted as described previously (31). Reactions contained $2 \mu \mathrm{mol} / \mathrm{L}$ actin (10\% pyrenelabeled), $75 \mathrm{nmol} / \mathrm{L}$ Arp2/3 complex, $100 \mathrm{nmol} / \mathrm{L}$ cortactin, or $50 \mathrm{nmol} / \mathrm{L}$ GST-VCA (\#VCG03; Cytoskeleton), and/or varying amounts of CK2 $\alpha$ (\#14-445; Millipore) as indicated. For reactions with CK2 $\alpha$, GST-VCA, or cortactin mutants were preincubated with CK2 $\alpha$ and $500 \mathrm{nmol}$ ATP for 15 minutes at room temperature prior to addition to the actin polymerization reaction.

\section{PDX-derived cell lines}

Patient-derived xenograft (PDX) tumors and cell lines were established as described (32). WVUSCC-AR2 and WVUSCC-AR5 were derived from surgical specimens of alveolar ridge HNSCC in compliance with West Virginia University Institutional Review Board approved protocol \#1310105737A033. PDXs were developed in compliance with West Virginia University Institutional Animal Care and Use Committee approved protocol \#15-0302.6 by placing approximately $1 \mathrm{~mm}$ tumor fragments into subcutaneous pockets in the flanks of anesthetized 8- to 10-week-old NOD/SCID- $\gamma$ (NSG) mice. Tumor fragments were overlayed with Matrigel (354234; Corning) and incisions were closed using wound clips. Mice were weighed and monitored for tumor growth on a weekly basis. PDX tumors were passed into new NSG mice and/or used to generate cell lines once tumors reached $\sim 1 \mathrm{~cm}$ in greatest dimension.

For cell line derivation, PDX tumors were minced and digested in DMEM supplemented with $20 \%$ FBS and $1 \mathrm{mg} / \mathrm{mL}$ collagenase IV (17104019; Gibco). Digested tissues were plated onto NIH3T3 fibroblasts senesced with $4 \mu \mathrm{g} / \mathrm{mL}$ mitomycin C (BP2531; Fisher Scientific) and cultured in DMEM:F12 1:1 supplemented with $10 \%$ FBS, $400 \mathrm{ng} / \mathrm{mL}$ hydrocortisone (H0888; Sigma), $50 \mu \mathrm{g} / \mathrm{mL}$ gentamycin (15750060; Gibco), $5 \mu \mathrm{mol} / \mathrm{L}$ ROCK inhibitor (S1049; Selleckchem), $0.5 \mathrm{ng} / \mathrm{mL}$ recombinant human EGF (PHG0311; Gibco), and $10 \mathrm{ng} / \mathrm{mL}$ cholera toxin (C8062; Sigma). Both WVUSCC-AR2 and -AR5 were derived in August 2017 and maintained for $\leq 10$ passages. Derived lines were verified using cytokeratin 14 staining (ab15462; Abcam). Neither STR profiling nor mycoplasma detection was performed on these cell lines. Prior to utilization in gelatin degradation or spheroid invasion assays, PDX-derived cell lines were plated directly onto cell culture dishes for one to two passages to remove the fibroblast population. Gelatin degradation and spheroid invasion assays were performed in DMEM supplemented with 10\% FBS.

\section{In vitro tumor spheroid invasion}

$3 \mathrm{D}$ spheroid invasion assays were performed as previously described (26). A total of $1 \times 10^{4}$ (OSC19) or $2.5 \times 10^{4}$ (UMSCC1 and WVUSCC-AR5) cells were plated into individual wells coated with $1.5 \%$ noble agar for 24 hours (UMSCC1) or 48 hours (OSC19 and WVUSCC-AR5) to form spheroids. For each line, spheroids were collected, resuspended in $500 \mu \mathrm{L}$ of $2 \mathrm{mg} / \mathrm{mL}$ rat tail collagen I (354236; Corning), and plated into individual wells 
of a 24 -well plate precoated with $400 \mu \mathrm{L}$ solidified $2 \mathrm{mg} / \mathrm{mL}$ collagen I. Plates were incubated for 1 hour at $37^{\circ} \mathrm{C}$, then overlayed with $1 \mathrm{~mL}$ DMEM supplemented with $10 \%$ FBS and $1 \%$ penicillin-streptomycin containing DMSO or $10 \mu \mathrm{mol} / \mathrm{L}$ Silmitasertib. Spheroid invasion was visualized at the indicated time points by phase contrast microscopy using a Zeiss Axiovert 200M microscope equipped with an AxioCamMR CCD camera using a Plan-Neofluar 5X/0.15 objective and Axiovision software (Carl Zeiss Microscopy). Maximal radial distances for invaded cells were calculated using Axiovision software, with invasive distance determined as the difference between the initial and final maximum radius for each invaded spheroid.

Orthotopic tongue tumors and invasion analysis

Tongue tumor establishment was adapted from previous work (33). A total of $2.5 \times 10^{4}$ luciferase expressing UMSCC1 cells were injected into the tongues of 8- to 10-week-old NSG mice (purchased from the West Virginia University Transgenic Animal Core Facility). Mice were maintained using transgenic dough diet (S3472; Bioserve) and weighed every 2 to 3 days. Tumor growth was monitored by bioluminescent imaging using $150 \mathrm{mg} / \mathrm{kg}$ D-luciferin (122796; Caliper Life Sciences) injected intraperitoneally, followed by in vivo whole-body bioluminescence imaging using an IVIS Lumina-II system and Living Image 4.0 software (PerkinElmer). Tumors were allowed to establish for 1 week, then mice were divided equally into two groups based on approximate tumor size. Mice were given $50 \mathrm{mg} / \mathrm{kg}$ Silmitasertib in DMSO or DMSO alone by oval gavage twice daily for 3 weeks. Mice were subsequently euthanized, tongues excised, processed, and stained for histologic analysis.

To quantify invasion parameters, whole tongue histologic images were cropped to encompass the tumor invasive front and analyzed using ImageJ. Images were processed with the colour deconvolution 1.5 plugin using H\&E or H\&E2 presets. Resultant colour_1 images were 25\% contrast enhanced before conversion into binary images. ROIs were selected for particles above 15,000 pixel units and manually verified by overlay onto the original $\mathrm{H} \& \mathrm{E}$ image to remove artifacts. Invasive protrusions were defined as projections at the leading edge of the tumor surrounded by stroma on three sides and identified on the binary image using the polygon selection tool. Invasive distance was determined as the difference between the farthest edge of the protrusion and the protrusion base.

\section{Statistical analysis}

Differences in mean values between groups were evaluated using Student or Welch $t$ test. Significance was determined at $P<0.05$ utilizing GraphPad Prism 7 software. Error bars represent \pm SEM.

\section{Results}

Cortactin threonine 24 is required for Arp2/3 complex binding and activation

Phosphorylation of serine (S) 11, T13, and T24 in the murine cortactin NTA domain has been reported (19). The proximity of these residues to the canonical Arp2/3 binding motif consisting of amino acids 20-22 (DDW) have the potential to regulate Arp2/3 binding (Fig. 1A). To determine if these residues influence cortactin binding to Arp2/3, FLAG-tagged murine cortactin constructs were generated that contained serine to alanine (S11A) and threonine to alanine (T24A) phosphorylation-null mutations. T13 was not evaluated because it is not conserved in human cortactin. Co-immunoprecipitation studies indicate that S11A cortactin bound endogenous Arp2/3 at levels similar to WT cortactin, whereas T24A cortactin failed to effectively bind Arp2/3 despite retaining the DDW binding motif (Fig. 1B). Threonine to aspartic acid (T24D) phosphomimetic cortactin bound Arp2/3 at reduced levels compared with WT (Fig. 1B). These data demonstrate that both the DDW motif and T24 are required for optimal Arp2/3 complex binding. Furthermore, reduced Arp2/3 binding resultant from the addition of negative charge at amino acid 24 (T24D) suggests that phosphorylation may play a negative-regulatory role.

To assess the impact of T24 on Arp2/3 actin nucleation, recombinant human WT, $\triangle \mathrm{DDW}$, and T24A cortactin proteins were expressed in bacteria and purified (Fig. 1C). When evaluated in pyrene-labeled actin assembly assays, WT cortactin displayed slower polymerization kinetics compared with the N-WASp VCA domain, whereas the $\triangle \mathrm{DDW}$ mutant failed to activate Arp2/3 as previously reported (Fig. 1D; refs. 16, 34, 35). T24A cortactin demonstrated intermediate activity, with reduced nucleation levels compared with WT cortactin and increased nucleation compared with $\Delta$ DDW (Fig. 1D). Taken together these data identify T24 in the cortactin NTA as a critical residue required for optimal cortactin-mediated Arp2/3 binding and activation regardless of phosphorylation status.

\section{Cortactin T24 is required for invadopodia-mediated ECM degradation}

Cortactin is essential for initiating invadopodia formation, maturation, and ECM degradation in part due to NTA-mediated Arp2/3 binding $(36,37)$. To determine the role of cortactin T24 in invadopodia function, a panel of cortactin knockdown-rescue cell lines stably expressing FLAG-cortactin mutant constructs were produced in invasive UMSCC1 (Fig. 2) and OSC19 (Supplementary Fig. S1) HNSCC cell lines. Both lines spontaneously produce invadopodia and degrade ECM $(26,38)$. Although individual cortactin siRNA (siCTTN) and shRNA (shCTTN) treatment resulted in decreased cortactin expression and matrix degradation in each case (Fig. 2D; Supplementary Figs. S1B and S2), sequential exposure to cortactin siRNA in stable shRNA cells resulted in efficient and reliable cortactin knockdown (KD; Fig. 2; Supplementary Figs. S1 and S2). Cortactin KD cells were used for subsequent experimentation to minimize the possibility of residual endogenous cortactin masking the effects of re-expressed FLAG-cortactin mutants. FLAG-WT cortactin expression in KD cells partially restored the amount of active invadopodia formation in UMSCC1 cells (Fig. 2A-C) and fully restored ECM degradation in UMSCC1 (Fig. 2A and B) and OSC19 (Supplementary Fig. S1A-S1C) cell lines. FLAG- $\triangle D D W$ enhanced invadopodia precursor formation but failed to rescue active invadopodia and ECM degradation (Fig. 2A-C; Supplementary Fig. S1A-S1C). Similarly, both FLAG-T24A and FLAG-T24D cortactin restored invadopodia precursor formation while failing to induce invadopodia maturation above KD levels, with active invadopodia and ECM degradation levels for both mutants similar to that of FLAG- $\Delta D D W$ cortactin (Fig. 2A-C; Supplementary Fig. S1A, S1C, and S1D). These results suggest that Arp2/3 binding and activation facilitated by cortactin T24 is required for effective cortactinmediated invadopodia formation and ECM degradation in HNSCC cells. 
Figure 1.

Cortactin $\mathrm{T} 24$ is required for binding and activation of Arp2/3 complex.

A, Diagram representing cortactin

functional domains. NTA, N-terminal acidic domain; R1-R6, repeats regions with $\mathrm{F}$-actin binding site indicated; Helix, alpha helical domain, PRR, proline rich region; SH3, Src homology 3 domain. NTA domain with position of S11 and T24 in context of the DDW region is shown. B, Immunoprecipitation analysis of Arp $2 / 3$ binding to cortactin NTA mutants. HEK293T/17 cells transfected with FLAG-empty vector (EV), FLAG-wild-type cortactin (WT) or the indicated FLAG-cortactin mutants. Immune complexes were Western blotted with antibodies against cortactin (top) and Arp3 (bottom). 1:10 diluted total cell lysates were Western blotted as indicated. C, Coomassie blue staining of the indicated purified recombinant human cortactin proteins. D, Effect of cortactin T24A on Arp2/3 complex activation. Fluorometric evaluation of actin polymerization over time with the indicated cortactin mutants incubated with Arp2/3 complex and pyrene-labeled actin. Polymerization curves: WT cortactin (blue), T24A cortactin (dark green), and $\triangle \mathrm{DDW}$ cortactin (purple). N-WASp VCA domain (black) was used as a positive control; negative controls include Arp2/3 complex plus actin (pale green) and actin alone (red). Polymerization curves are representative from three independent experiments.
A

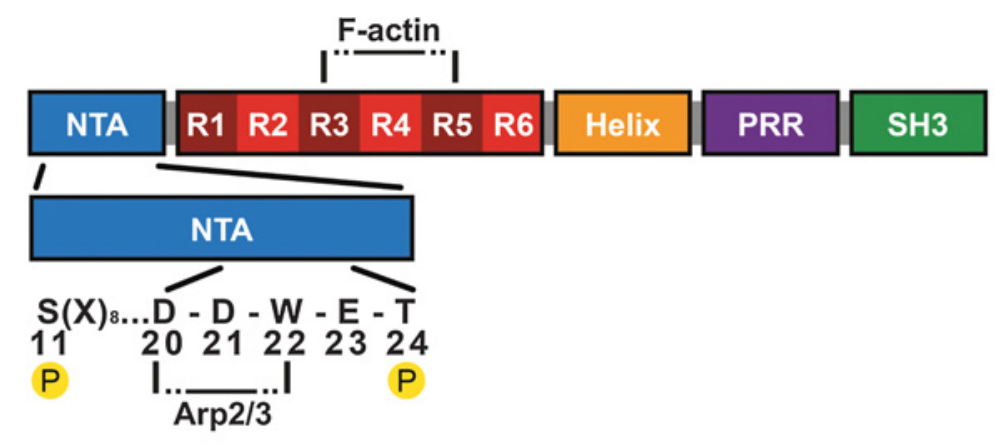

B

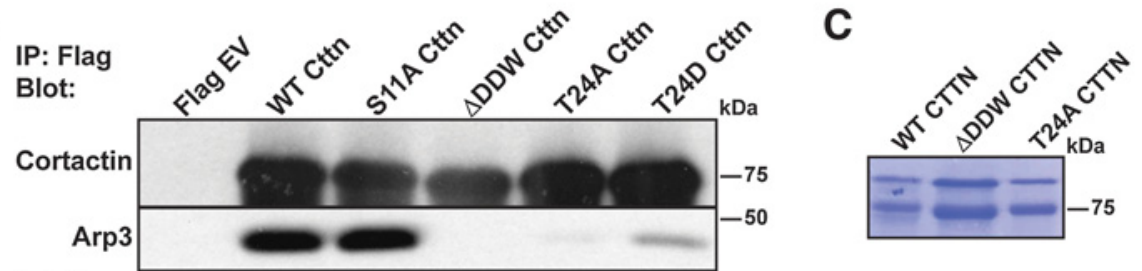

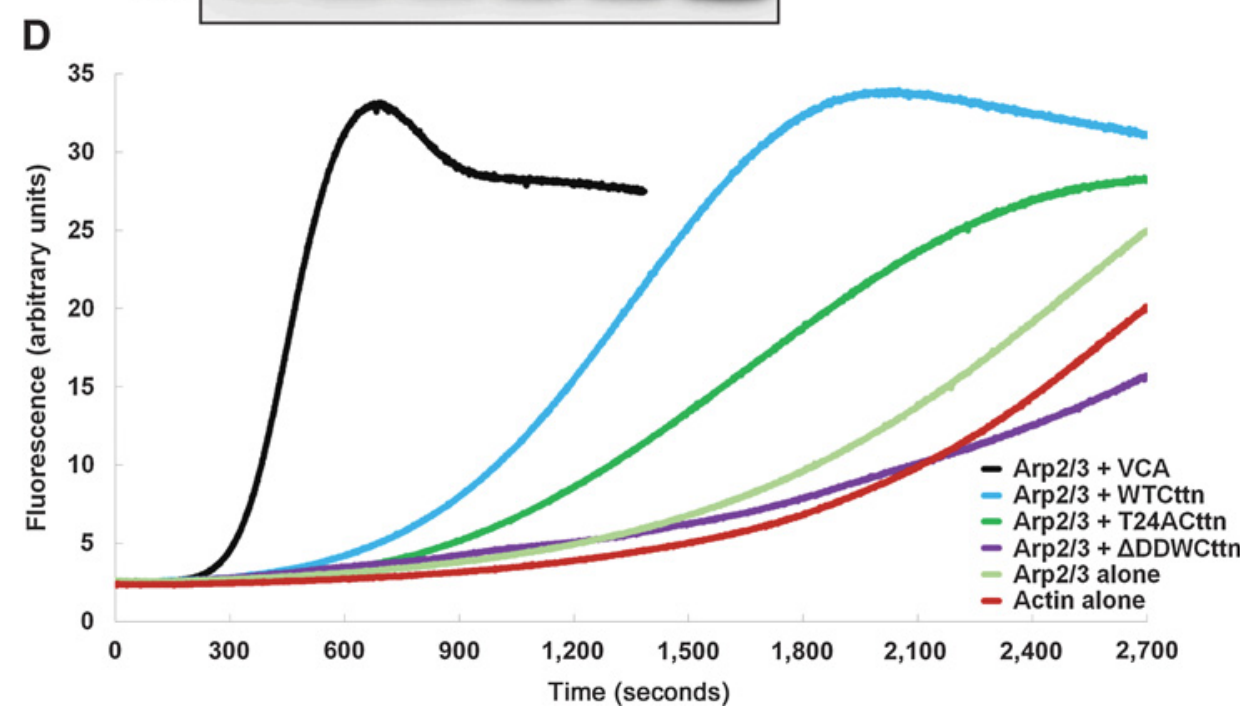

CK2 phosphorylation of cortactin T24 regulates interaction with Arp2/3 complex

The importance of T24 in Arp2/3 activation and invadopodia function, along with prior identification of T24 as a cortactin phosphorylation site, led us to identify the kinase(s) responsible for phosphorylating T24. Computational analysis of the sequences flanking T24 was performed by seven independent predictive algorithms, six of which suggested that CK2 $\alpha$ had the highest probability of phosphorylating cortactin T24 (Supplementary Table S1). To test this, kinase assays were conducted using GST-tagged cortactin WT and T24A NTA fusion proteins with purified active CK2 $\alpha$. The N-WASp VCA domain was used as a positive control, because previous studies have shown this region to be a CK2 $\alpha$ substrate $(23,25)$. Increasing amounts of GST-WT-NTA were efficiently phosphorylated by CK2 $\alpha$, whereas no phosphorylation was evident in GST-T24A-NTA (Fig. 3A). These data indicate that cortactin T24 can serve as a CK2 $\alpha$ substrate, and that $\mathrm{T} 24$ is the only residue targeted by CK2 $\alpha$ in the NTA region.

To determine if CK2 $\alpha$ phosphorylation of cortactin T24 effects binding to Arp2/3 complex, recombinant human WT or T24A cortactin proteins were pre-incubated with or without 
A
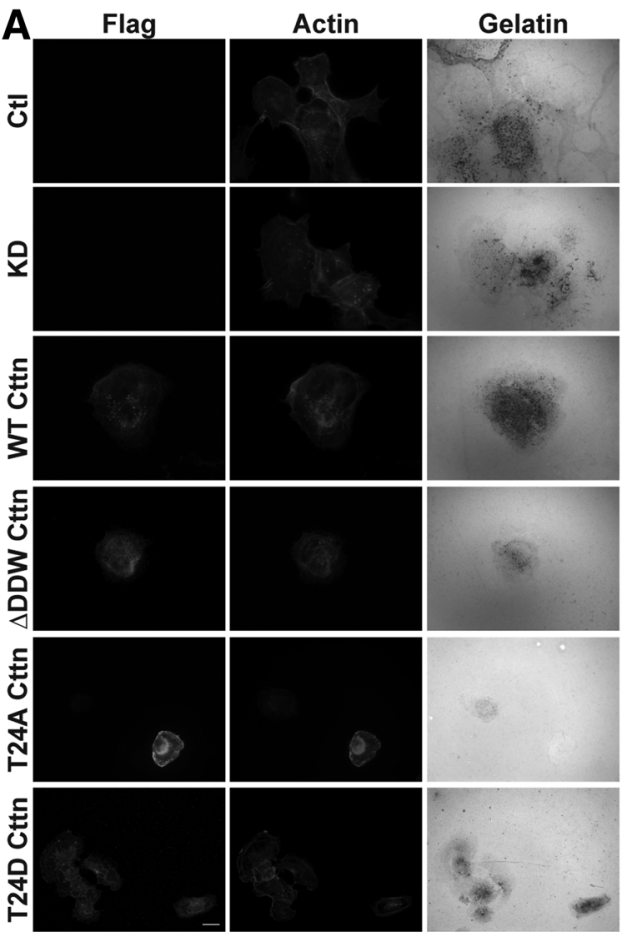

UMSCC1

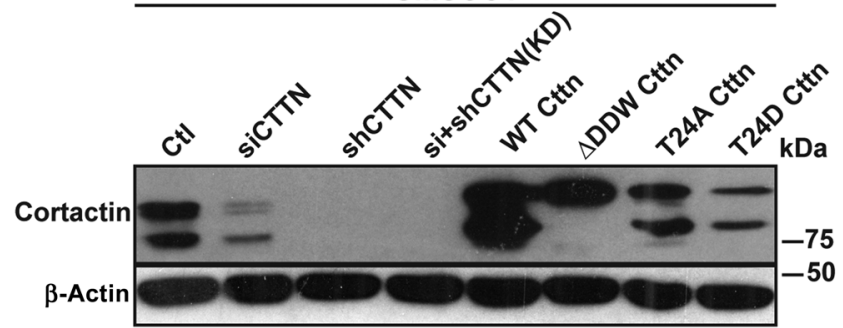

B

C
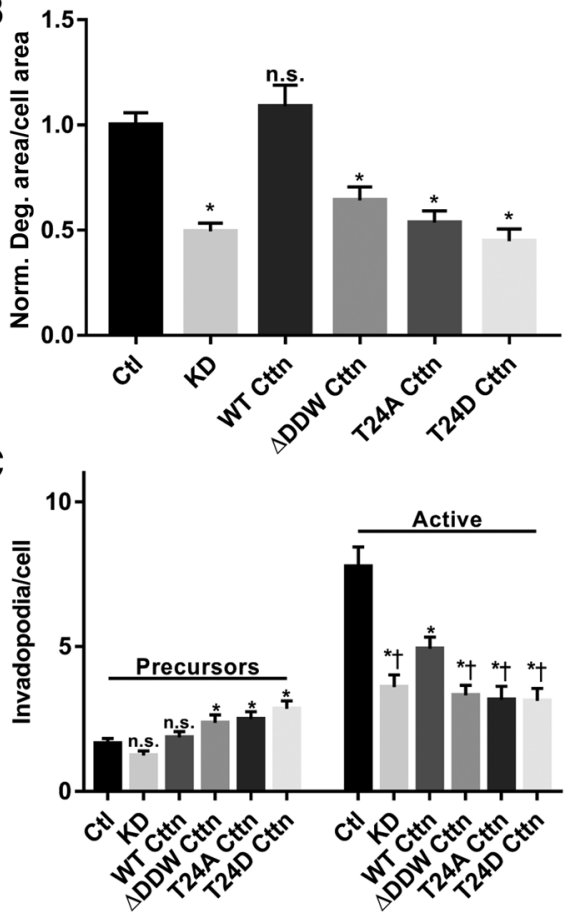

Figure 2.

T24 is required for cortactinmediated invadopodia formation and ECM degradation in HNSCC cells. A, UMSCC1 cells with stable shRNA scramble control (Ctl) or anti-cortactin shRNA combined with siRNA knockdown (KD) were transduced with murine FLAG-WT $-\triangle \mathrm{DDW},-\mathrm{T} 24 \mathrm{~A}$, and $-\mathrm{T} 24 \mathrm{D}$

cortactin lentiviruses. Cells were plated on Oregon Green (OG)-488 gelatin coated coverslips for 12

hours, fixed, and labeled with antiFLAG and rhodamine phalloidin (Actin). Gelatin panels are pseudocolored white; degradation is evident as black areas indicating loss of fluorescence. Scale bar represents $20 \mu \mathrm{m}$. B, Quantification of gelatin matrix degradation for control $(\mathrm{Ctl})$, cortactin $\mathrm{KD}$, and FLAG-cortactin expressing UMSCC1 cells. C, Quantification of invadopodia precursors (left) and active invadopodia (right) numbers from the lines assayed in $\mathbf{B}$. Data represents the mean + SEM of $n \geq$ 100 cells for each line analyzed from at least three independent experiments. All gelatin degradation conditions were normalized to $\mathrm{CtI}$ UMSCC1 cells. n.s., not significant; ${ }^{*}, P<0.05$, Welch's or Student $t$ test vs. Ctl (B, C); $\dagger, P<0.05$, Student $t$ test vs. wild type cortactin rescue (WT;C). D, Total cell lysates from (A) evaluated for endogenous and FLAG-cortactin expression by immunoblotting with antibodies against cortactin (top) and $\beta$-actin (bottom).

CK2 $\alpha$, then mixed with purified Arp2/3. Phosphorylation of WT cortactin by CK $2 \alpha$ reduced binding of Arp $2 / 3$ complex to background levels (beads alone) whereas no impact on T24A was observed (Fig. 3B). To ascertain the impact of CK2 $\alpha$ phosphorylation on cortactin-mediated Arp2/3 activation, actin assembly assays were conducted with CK $2 \alpha$-phosphorylated cortactin and N-WASp VCA domain. As previously determined, CK2 $\alpha$ phosphorylation of N-WASp VCA results in a modest reduction of Arp $2 / 3$ NPF activity (black vs. grey, Fig. 3C; ref. 25). Similarly, WT cortactin incubated with increasing amounts of $\mathrm{CK} 2 \alpha$ prior to inclusion in polymerization assays resulted in a dose-dependent suppression of actin assembly, suggesting that CK2 $\alpha$ phosphorylation impairs the ability of cortactin to activate Arp $2 / 3$ complex (Fig. 3C). Although Arp2/3 can be activated by direct phosphorylation from multiple kinases (39-41), CK2 $\alpha$ had no direct effect on Arp $2 / 3$ activation (orange vs. pale green, Fig. 3C), suggesting that the inhibitory effect on Arp $2 / 3$ activity is due to phosphorylated cortactin in these assays. To determine if the CK2 $\alpha$ targeted T24 residue is responsible for the observed inhibitory effect on Arp2/3 activity, polymerization assays were conducted with WT and T24A cortactin proteins following incubation with
CK2 $\alpha$. Although CK2 $\alpha$ inhibited the ability of WT cortactin to activate Arp2/3 complex (blue vs. red, Fig. 3D), preincubation of CK $2 \alpha$ with T24A cortactin exhibited no additional inhibitory effect on Arp2/3 nucleation activity (green vs. purple, Fig. 3D). Collectively these data indicate that cortactin phosphorylation at T24 by CK2 $\alpha$ reduces the ability of cortactin to bind and activate Arp $2 / 3$ complex-mediated branched actin network formation.

CK2 $\alpha$ is required for optimal HNSCC invadopodia function

To determine if $\mathrm{CK} 2 \alpha$ impacts invadopodia function, CK2 $\alpha$ expression was stably knocked down in OSC19 and UMSCC1 cells using anti-CK2 $\alpha$ shRNAs targeting two different regions in the CK2 $\alpha$ transcript. Both shRNAs reduced CK2 $\alpha$ expression to nondetectable levels in each cell line (Fig. 4A). Neither line expressed the alternative CK2 $\alpha^{\prime}$ isoform in control or CK2 $\alpha$ knockdown cells (data not shown). CK2 $\alpha$ knockdown in OSC19 cells reduced the level of active invadopodia by $71 \%$ to $88 \%$ and ECM degradation by $63 \%$ to $73 \%$, whereas knockdown in UMSCC 1 cells reduced active invadopodia formation by $68 \%$ to $92 \%$ and ECM degradation by $36 \%$ to $60 \%$ (Fig. 4B-D). Although these data indicate that CK2 $\alpha$ is a key mediator of invadopodia 
Figure 3.

CK2 $\alpha$ phosphorylation of cortactin T24 inhibits Arp2/3 complex binding and activation. A, Cortactin T24 is a CK2 $\alpha$ phosphorylation site. Autoradiogram of active CK2 $\alpha$ incubated with the increasing amounts $(0,0.25,0.5$, and $1 \mu \mathrm{g})$ of GST-WT-NTA or GST-T24A-NTA cortactin fusion proteins. GST $(1 \mu \mathrm{g})$ and the GST-VCA domain of N-WASp $(0.25 \mu \mathrm{g})$ were used as respective negative and positive phosphorylation controls. Positions of autophosphorylated CK2 $\alpha$, GST-VCA, and cortactin NTA proteins are indicated on the left; autoradiogram is representative of three independent experiments.

B, CK2 $\alpha$ phosphorylation at cortactin T24 ablates binding to Arp2/3 complex. Purified recombinant human WT and T24A cortactin proteins $(2.5 \mu \mathrm{g})$ were bound with an anti-cortactin antibody to protein $\mathrm{G}$ beads. Immune complexes were

preincubated with or without $75 \mathrm{ng}$ active $C K 2 \alpha$, washed and incubated with 50 ng purified Arp2/3

complex. Co-immunoprecipitated complexes were Western blotted for cortactin (top) and Arp3 (bottom). 4F11-bound protein G beads were used as a negative control for nonspecific binding (Beads). Arp2/3 complex (5 ng) was used as positive control for Arp3 immunoblotting. Blot is representative of two independent experiments. C, Cortactin phosphorylation by CK2 $\alpha$ inhibits cortactin-mediated Arp2/3 actin polymerization. WT human cortactin or GST-VCA proteins were preincubated with the indicated amounts of active CK $2 \alpha$ and evaluated for effects on Arp2/3 activity. Polymerization curves are representative from three independent experiments. D, Phosphorylation of T24 is responsible for the inhibitory effect of CK $2 \alpha$ on cortactin-mediated Arp2/3 activation. Human WT and T24A cortactin proteins were preincubated with or without $30 \mathrm{ng}$ active $\mathrm{CK} 2 \alpha$ and evaluated for effects on Arp2/3-mediated actin assembly. Polymerization curves are representative from three independent experiments.
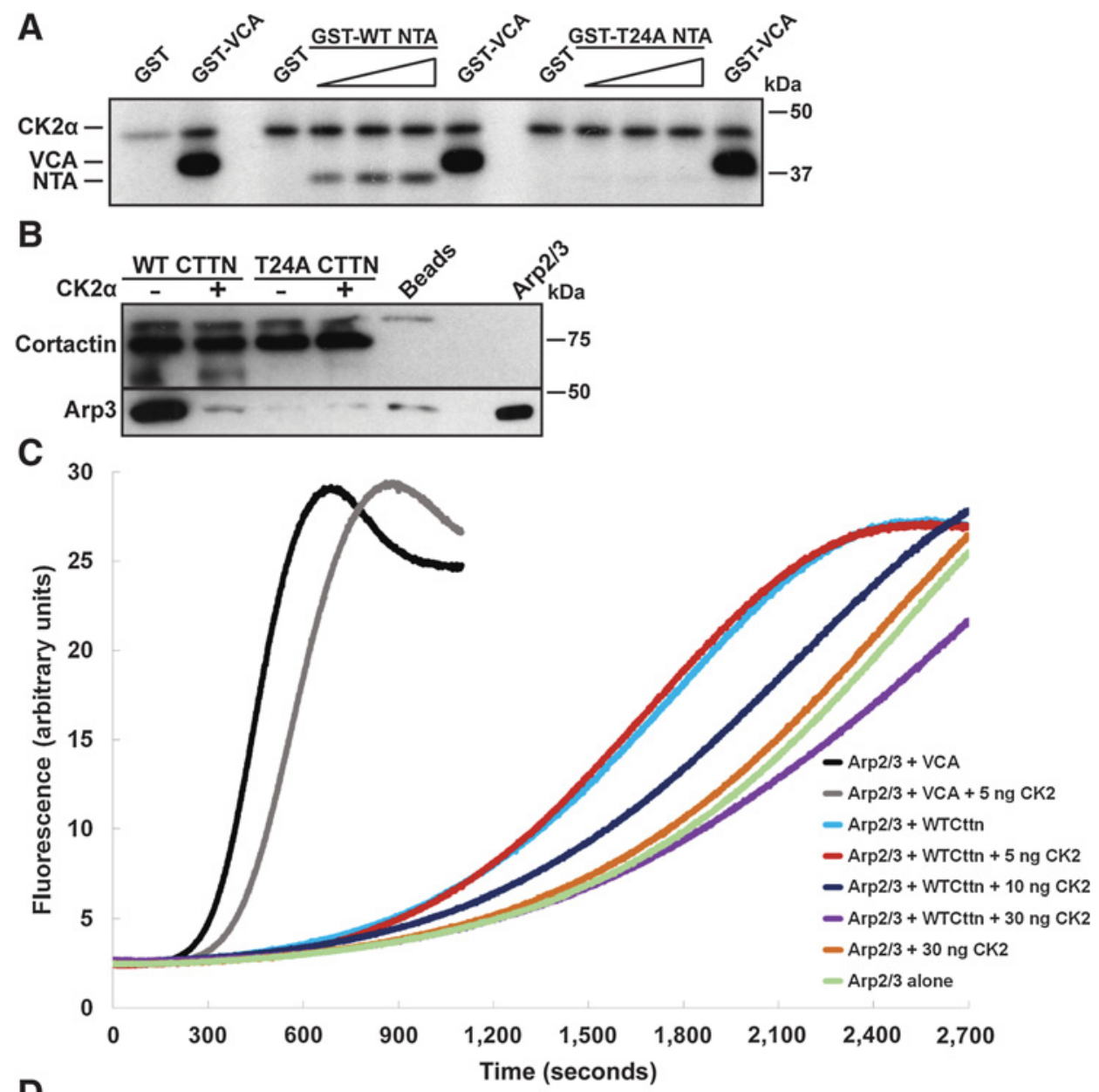

D

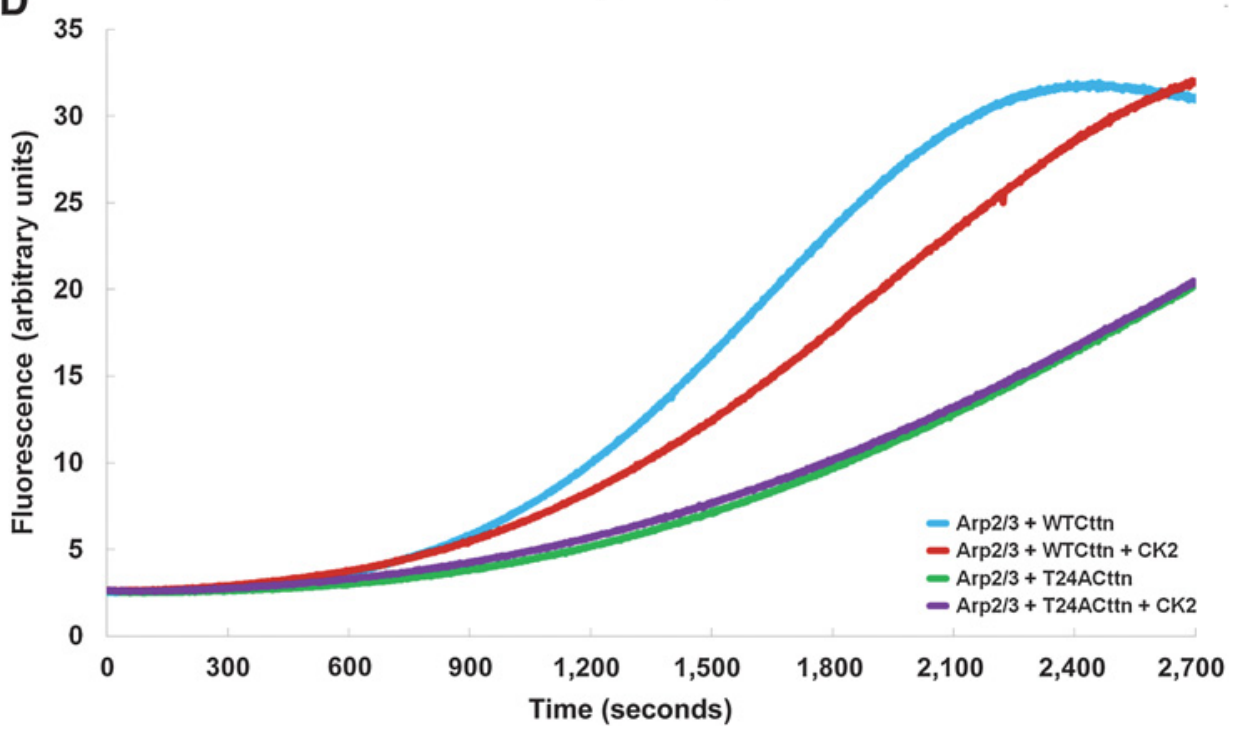

maturation and function, neither invadopodia formation nor matrix degradation was entirely abolished. This would indicate that alternative signaling pathways impinging on cortactin and other invadopodia proteins remain active in the absence of CK $2 \alpha$ expression.
The CK2 $\alpha$ inhibitor Silmitasertib suppresses invadopodia function in HNSCC cells

Silmitasertib (CX-4945) is an orally bioavailable small molecule ATP-competitive inhibitor that targets CK2 $\alpha$ kinase activity and is currently undergoing clinical trials in multiple cancer types 


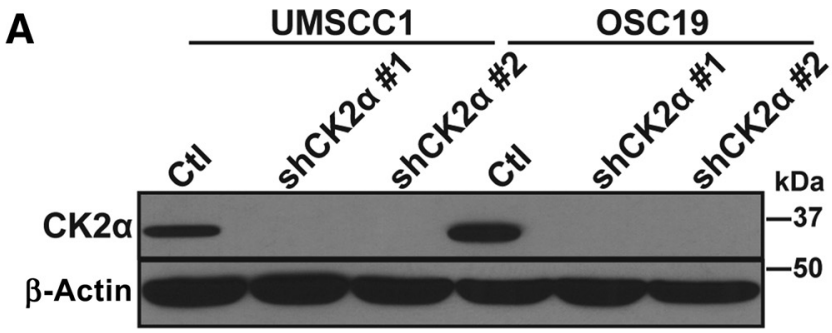

B

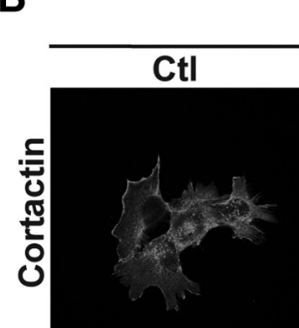

OSC19
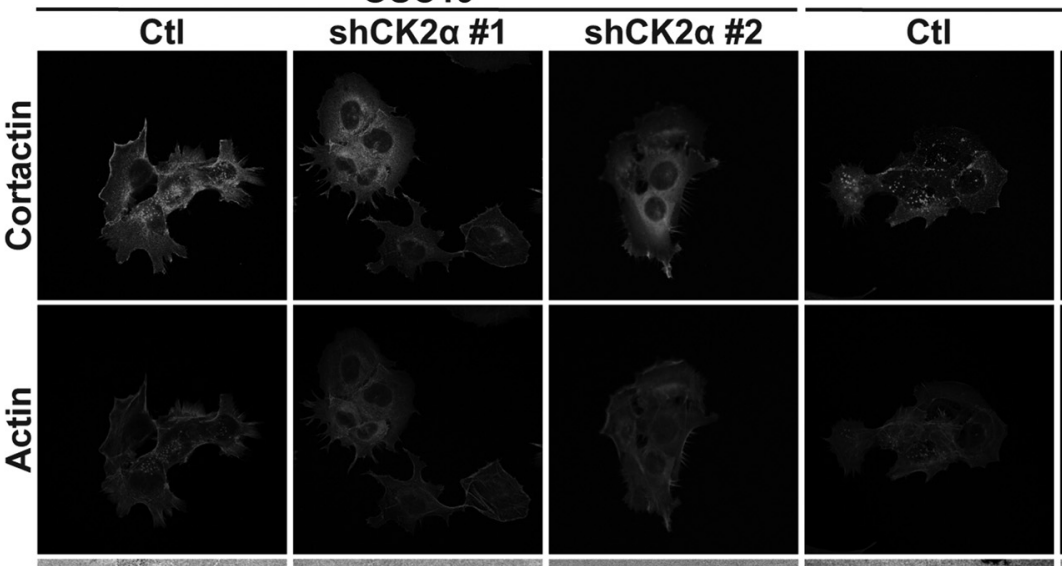

UMSCC1
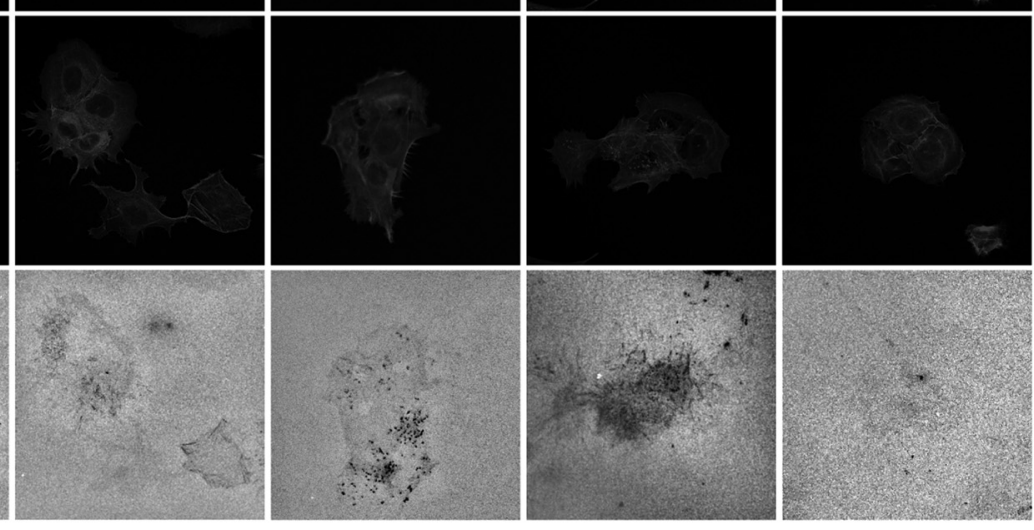

$\operatorname{shCK2} \alpha \# 2$
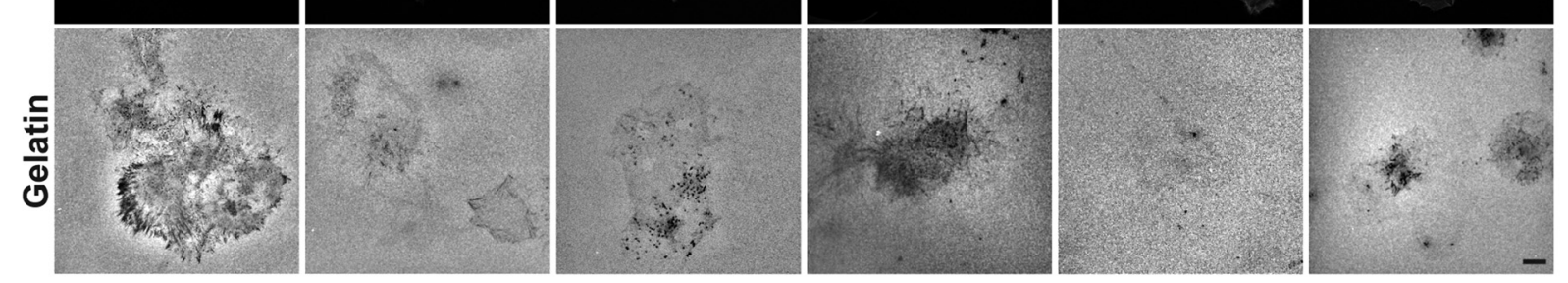

\section{C}
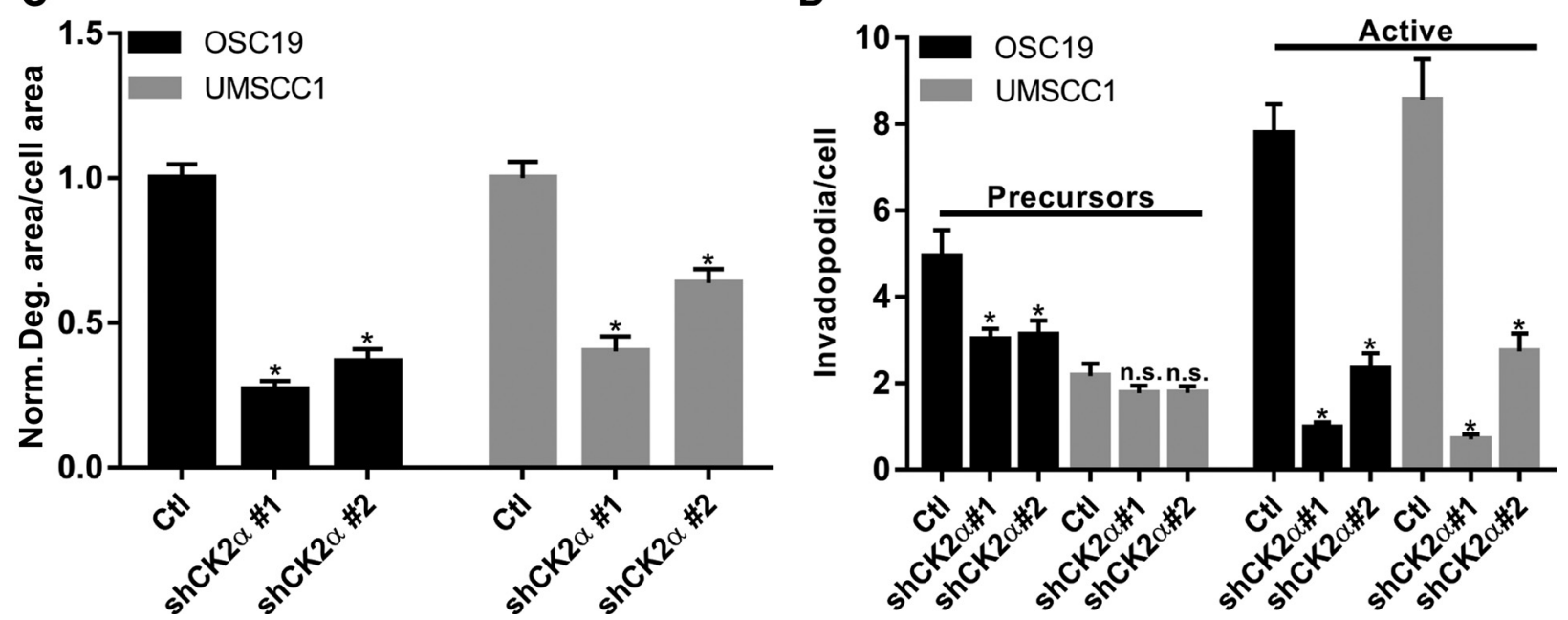

Figure 4.

CK2 $\alpha$ is required for optimal HNSCC invadopodia function. A, Evaluation of CK2 $\alpha$ expression in stable scramble control (CtI) and CK2 $\alpha$ shRNA HNSCC cells. Cells were lysed and Western blotted with antibodies against CK2 $\alpha$ (top) and $\beta$-actin (bottom). B, Representative confocal images of OSC19 and UMSCC1 cells with CtI shRNA and with each anti-CK2 $\alpha$ shRNA (shCK2 $\alpha \# 1$ and \#2). Cells were plated on OG-488 gelatin coverslips for 12 hours then labeled with an anti-cortactin antibody and rhodamine phalloidin (Actin). Gelatin is pseudo-colored white. Scale bar represents $20 \mu \mathrm{m}$. C, CK2 $\alpha$ knockdown decreases invadopodia-mediated ECM degradation. Quantification of matrix degradation area per cell area for Ctl and each anti-CK2 $\alpha$ shRNA in the indicated cell lines. Degradation data were normalized to $\mathrm{CtI}$ condition for each cell line. D, CK2 $\alpha$ knockdown decreases invadopodia numbers. Amount of invadopodia precursors (left) and active invadopodia (right) per cell is shown for control and shCK2 $\alpha$ OSC19 and UMSCC1 cells. Data in C and D represent the mean + S.E.M. of $n \geq 100$ cells analyzed from at least three independent experiments. n.s., not significant; ${ }^{*}, P<0.05$, Welch $t$ test vs. Ctl. 
(NCT01199718, NCT02128282, NCT00891280; ref. 20). To determine the impact of Silmitasertib on HNSCC tumor cellmediated invadopodia formation and ECM degradation, established HNSCC cell lines were treated with increasing Silmitasertib concentrations and evaluated for effects on invadopodia activity and ECM degradation. Dose-dependent decreases in ECM degradation were observed at concentrations above $0.5 \mu \mathrm{mol} / \mathrm{L}$ in all evaluated HNSCC lines (Fig. 5A and B). The greatest impairment of gelatin degradation was seen at $10 \mu \mathrm{mol} / \mathrm{L}$ (Fig. 5A and B), comparable to effective CK2-specific growth-inhibitory doses in several cancer cell lines $(20,42)$. At this concentration, active invadopodia formation was significantly diminished in OSC19 and UMSCC1 cells, whereas MDA1586 cells displayed nonsignificant decreases (Fig. 5C). To determine if the invadopodia inhibitory effect of Silmitasertib was directly due to altering CK2 $\alpha$-mediated cortactin T24 phosphorylation, antibodies against phosphorylated cortactin T24 peptides (pT24) were designed and purified. Attempts by two different commercial vendors failed to generate a pT24-specific antibody (not shown). Therefore, we evaluated the phosphorylation status of S473 (pS473) in AKT following Silmitasertib treatment as a surrogate marker for drug efficacy, as this site is known to be phosphorylated by CK2 $\alpha$ (20). MDA1586 cells treated with 1 or $10 \mu \mathrm{mol} / \mathrm{L}$ Silmitasertib had decreased pS473 AKT after 24 hours, the same timeframe used in ECM degradation assays (Supplementary Fig. S3). OSC19 and UMSCC1 cells had decreased pS473 AKT after treatment with $10 \mu \mathrm{mol} / \mathrm{L}$ Silmitasertib for 12 hours, the time used for matrix degradation assays in these lines (Supplementary Fig. S3).

The fact that CK2 $\alpha$ phosphorylates multiple targets aside from cortactin (20) raises the possibility that the inhibitory effect of Silmitasertib may be due to impairing phosphorylation of additional proteins involved in invadopodia function. To determine the extent of cortactin-specific CK2 $\alpha$ phosphorylation in invadopodia-mediated ECM degradation, UMSCC1 cells expressing FLAG-WT, FLAG-T24A, and FLAG-T24D cortactin were treated with $1 \mu \mathrm{mol} / \mathrm{L}$ Silmitasertib and evaluated for additional suppressive effects on matrix degradation (Fig. 5D and E). Silmitasertib diminished ECM degradation in cells expressing FLAG-WT cortactin by $51 \%$, similar to the reduction observed in nontransfected cells (Fig. 5D vs. B). Neither FLAG-T24A nor FLAG-T24D expressing cells treated with Silmitasertib demonstrated reductions in ECM degradation levels from baseline vehicle-treated controls (Fig. 5D and E). Although these results do not entirely negate alternative CK2-dependent signaling pathways in invadopodia regulation, it does suggest that cortactin T24 is the primary CK2 $\alpha$ target in governing HNSCC invadopodia function.

To evaluate the anti-invadopodia effect of Silmitasertib in more translationally-relevant models, PDXs were derived from a welland a moderately-differentiated HNSCC surgical sample. PDX tumors maintained original patient tumor architecture, displaying collective invasion and keratin pearls characteristic of differentiated HNSCC (Fig. 6A). Primary cell lines derived from these PDX tumors form invadopodia and spontaneously degrade gelatin within 24 hours (Fig. 6B). Both lines exhibit tight colony morphology under cell culture conditions consistent with HNSCC lines derived from epithelial HNSCC (Fig. 6B; refs. 32, 43). Treatment of WVUSCC-AR2 and WVUSCC-AR5 with Silmitasertib yielded similar results to those observed in established lines, with gelatin degradation impaired $38 \%$ to $62 \%$ in WVUSCC-AR2 cells and 56\% to $66 \%$ in WVUSCC-AR5 cells at and above $0.5 \mu \mathrm{mol} / \mathrm{L}$ (Fig. $6 \mathrm{C}$ and D). Similarly, $10 \mu \mathrm{mol} / \mathrm{L}$ Silmitasertib treatment suppressed active invadopodia by $76 \%$ in WVUSCC-AR2 and 91\% in WVUSCC-AR5 (Fig. 6E). Collectively these data indicate that $\mathrm{CK} 2 \alpha$ kinase activity is essential for maximal ECM degradation ability in HNSCC.

\section{Silmitasertib inhibits HNSCC invasion}

To determine if Silmitasertib impacts HNSCC invasion, we initially utilized a 3D in vitro assay designed to model collective invasion typically observed in differentiated HNSCC. Tumor spheroids generated from OSC19, UMSCC1, and WVUSCC-AR5 PDX lines were embedded between layers of collagen I. WVUSCCAR2 cells failed to form spheroids and could not be used in this assay. Spheroids were treated with $10 \mu \mathrm{mol} / \mathrm{L}$ Silmitasertib or vehicle (DMSO) for 48 hours (Fig. 7A and B). Silmitasertib significantly reduced $3 \mathrm{D}$ collective invasion in all assayed lines (Fig. 7B). We next evaluated the ability of Silmitasertib to control invasion in the tongues of mice harboring orthotopic tumors. Luciferase-expressing UMSCC1 cells injected into the tongues of NSG mice formed detectable tumors within 1 week (Fig. 7C). Mice were divided into two groups containing similar tumor size as determined by in vivo bioluminescence, with one group treated with vehicle and one with Silmitasertib. Mice dosed twice daily for 3 weeks displayed a nonsignificant reduction in tumor growth compared with controls, similar to previous single agent xenograft studies (Fig. 7C; ref. 20). To negate potential bias due to unequal tumor size, four equivalent tumors from each group were selected for further assessment. In-depth evaluation of tumor margins from tongues excised after 4 weeks revealed alterations in invasive characteristics of Silmitasertib-treated mice (Fig. 7D-I). Tumors in drug-treated mice had a less ragged appearance at the invasive front, exhibiting shorter and smaller collective cell protrusions into the tongue stroma compared with control mice (Fig. 7D-G). In addition, tongues from Silmitasertib-treated mice had reduced perineural invasion of nerves adjacent to the invasive front (Fig. $7 \mathrm{H}$ and I). No difference was seen in the size or invasive distance of detached collective groups within the invasive front (Supplementary Fig. S4). Collectively these data support a role for CK2 $\alpha$ signaling in driving several pro-invasive behaviors associated with poor patient outcome in $\operatorname{HNSCC}(2,44)$.

\section{Discussion}

Proteolysis of restrictive tissue barriers is essential to all steps in the metastatic cascade and increasing evidence indicates that the proteolytic activity of invadopodia is required for invasive breaching of ECM barriers (1). Signals that govern invadopodia are dynamic and highly regulated, requiring coordinated activity of several oncogenic pathways in parallel to achieve maximal efficiency (1). Active assembly and turnover of cellular F-actin networks is required for initiating invadopodia formation and subsequent maturation, involving recruitment and activation of membrane-bound and secreted MMPs to mediate ECM proteolysis (1). Breakdown of existing F-actin networks, in conjunction with Arp2/3 activation in invadopodia, is responsible for actin network turnover necessary for productive branched F-actin formation that drives membrane protrusion. Early recruitment of cortactin is necessary for invadopodia initiation, where phosphorylation of C-terminal tyrosines 421,470 , and 486, along with serines 405 and 418, occurs downstream of growth factor and integrin signaling (45). These phosphorylation events create 
Markwell et al.

A
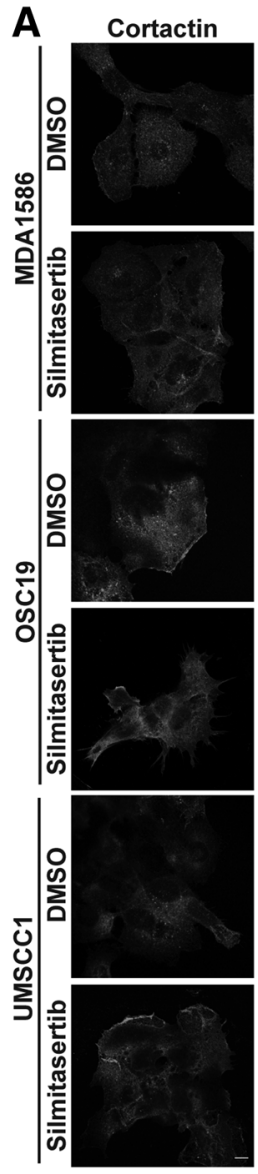

Actin
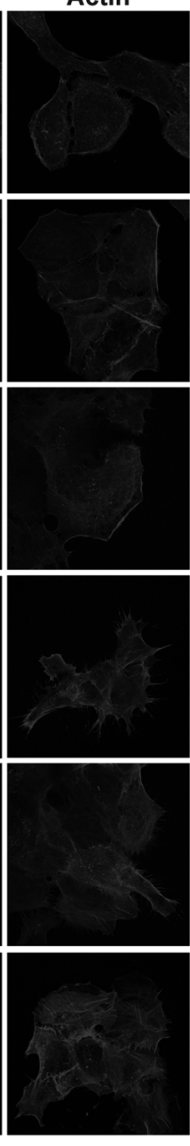

WT
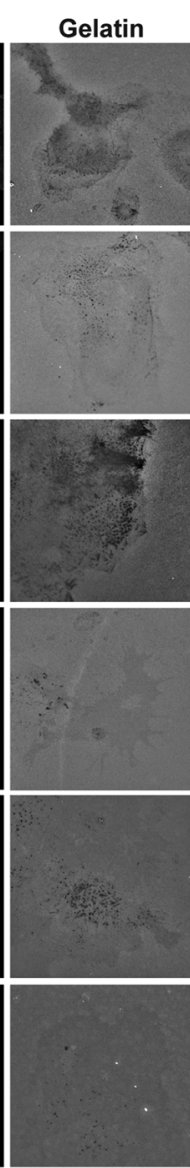

B

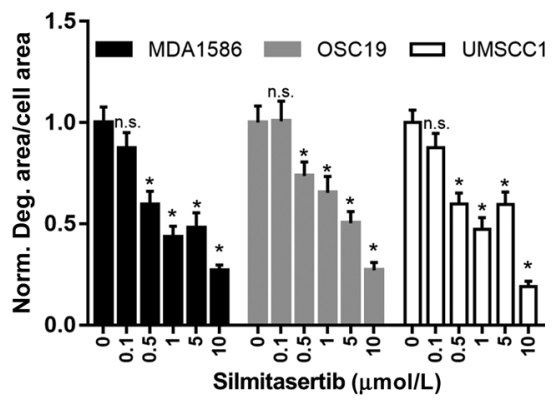

C
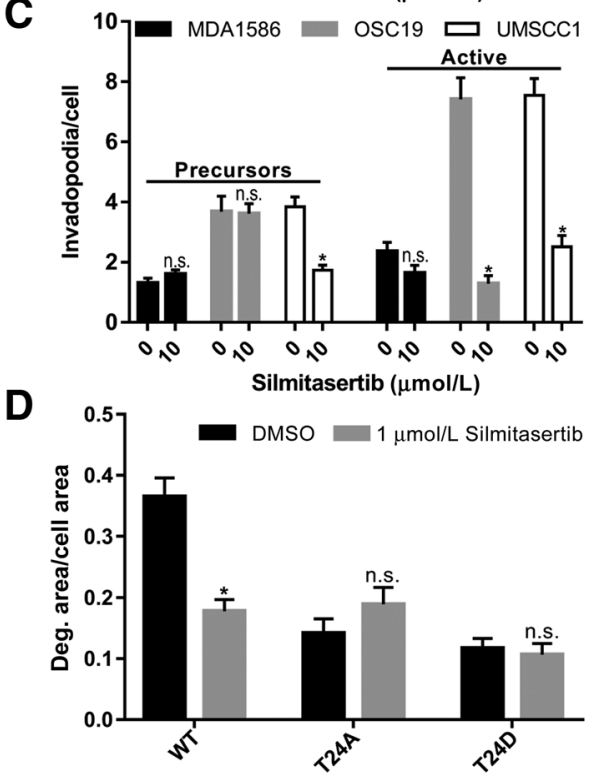

E

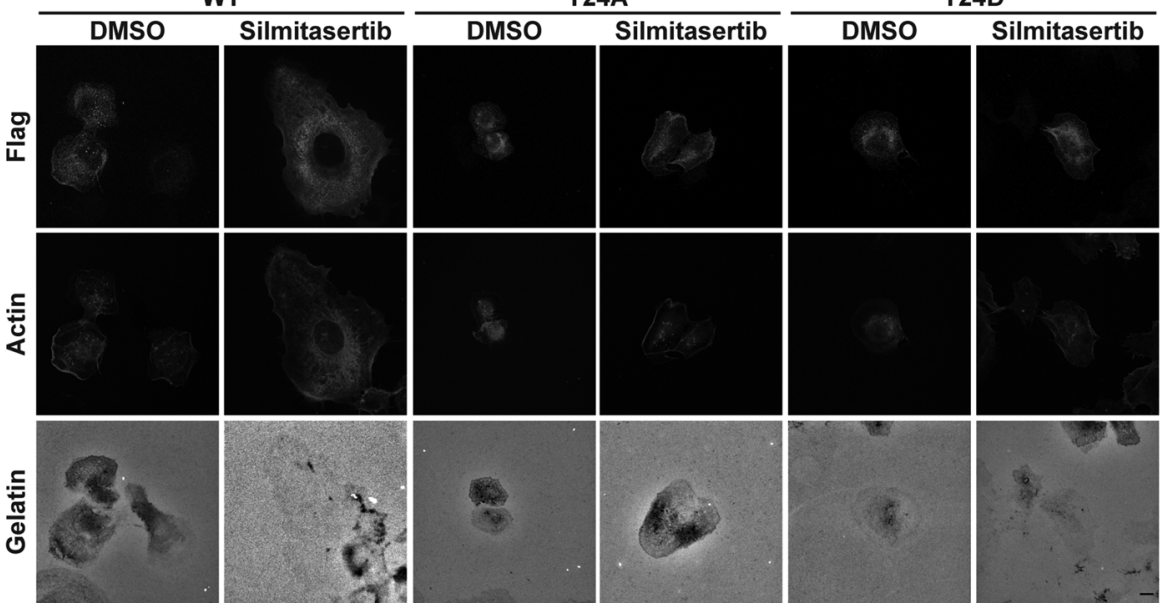

Figure 5.

Silmitasertib-mediated CK2 inhibition reduces invadopodia function in established HNSCC cell lines. A, Representative confocal images of MDA1586, OSC19, and UMSCC1 cells plated on OG-488 gelatin coverslips for 1 hour before treatment with vehicle (DMSO) or $10 \mu \mathrm{mol} / \mathrm{L}$ Silmitasertib for 24 hours (MDA1586) or 12 hours (OSC19 \& UMSCC1; optimal ECM degradation times for each line). Cells were fixed and labeled with an anti-cortactin antibody and rhodamine phalloidin (Actin). Gelatin is pseudo-colored white. Scale bar represents $20 \mu \mathrm{m}$. B, CK2 $\alpha$ inhibition decreases invadopodia-mediated ECM degradation Quantification of gelatin matrix degradation area per cell area in HNSCC cell lines treated with the indicated Silmitasertib concentrations. C, CK2 $\alpha$ inhibition decreases invadopodia numbers. Amount of invadopodia precursors (left) and active invadopodia (right) per cell is shown for Silmitasertib-treated HNSCC cell lines. Degradation data were normalized to DMSO-treated (0) cells for each cell line. Data in $\mathbf{B}$ and $\mathbf{C}$ represent the mean + SEM of $n \geq 100$ cells analyzed from at least three independent experiments. n.s., not significant; ${ }^{*}, P<0.05$, Welch or Student $t$ test vs. DMSO for each cell line. D, Quantitation of gelatin matrix degradation area per cell area for UMSCC1 cells expressing FLAG-WT, -T24A, or -T24D cortactin treated with DMSO or $1 \mu \mathrm{mol} / \mathrm{L}$ Silmitasertib. Data represent the mean + SEM of $n \geq 75$ cells analyzed from at least three independent experiments. n.s., not significant; ${ }^{*}, P<0.05$, Welch or Student $t$ test vs. DMSO for each condition. E, Representative confocal images of UMSCC1 cells expressing FLAG-WT, -T24A, or -T24D cortactin plated on OG-488 gelatin coverslips for 1 hour before treatment with vehicle (DMSO) or $1 \mu \mathrm{mol} / \mathrm{L}$ Silmitasertib for 12 hours. Cells were fixed and labeled with an anti-FLAG antibody and rhodamine phalloidin (Actin). Gelatin is pseudo-colored white. Scale bar represents $20 \mu \mathrm{m}$. 

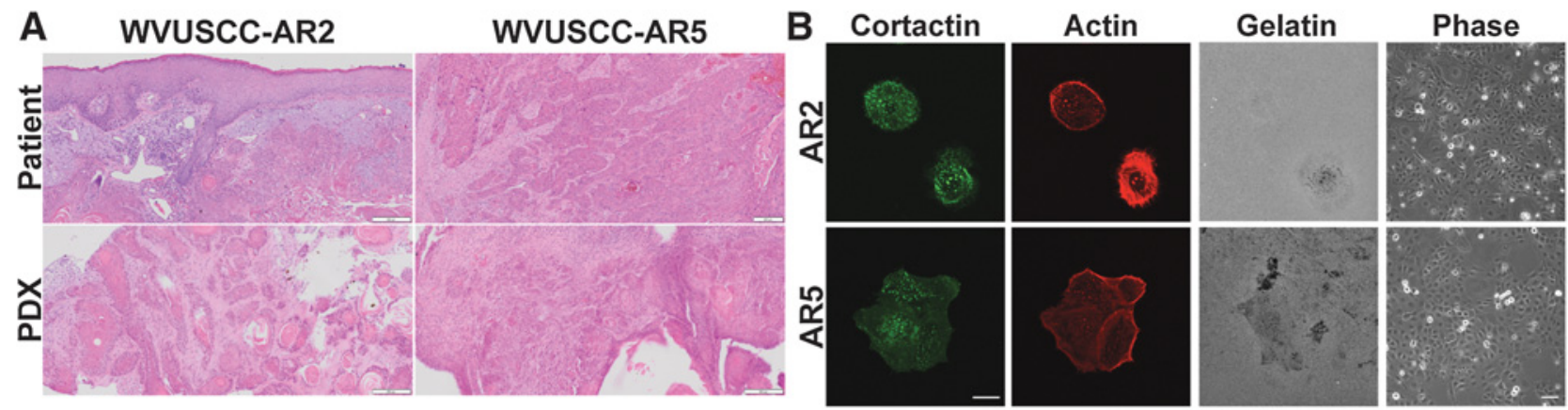

C
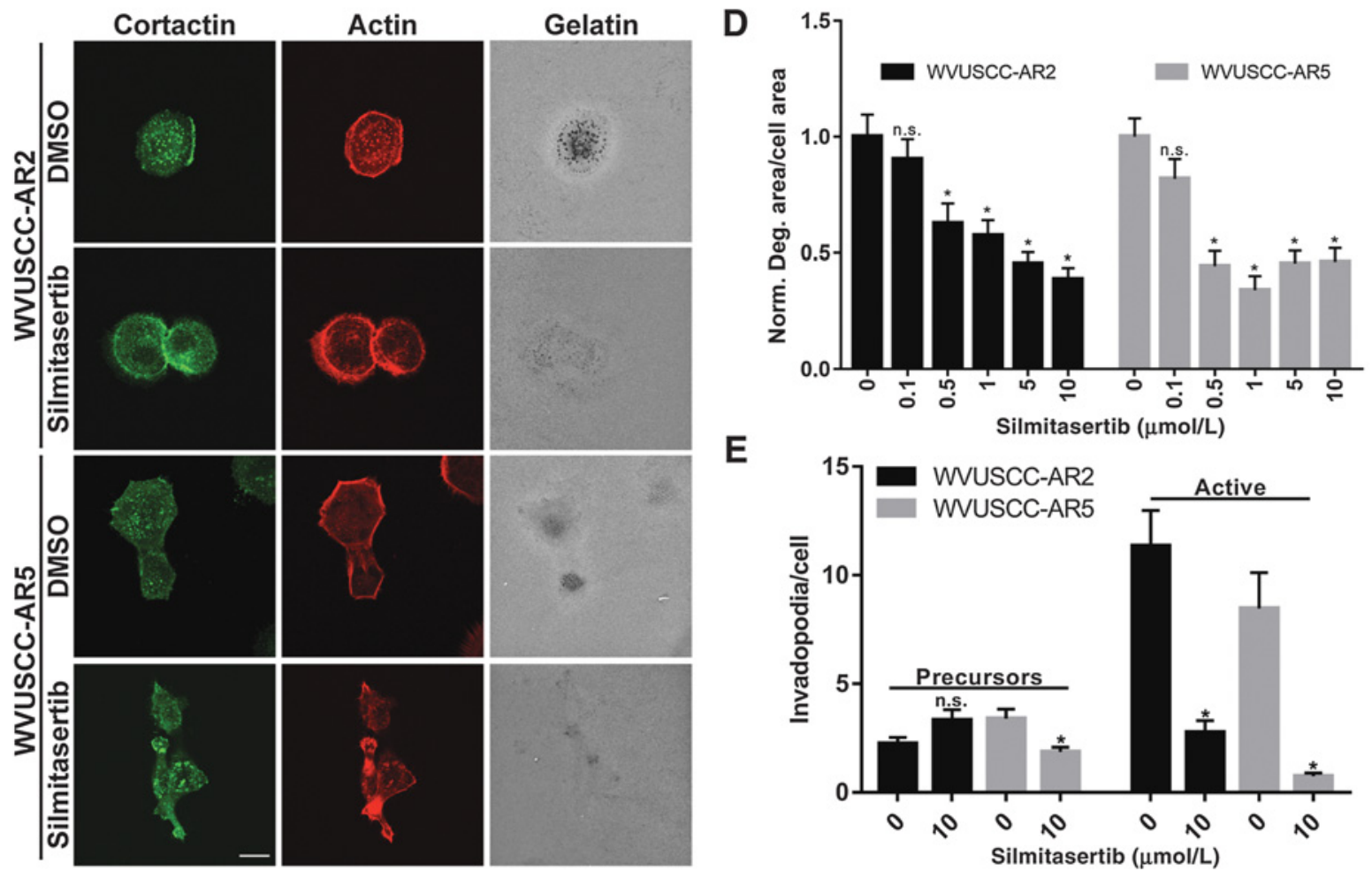

E

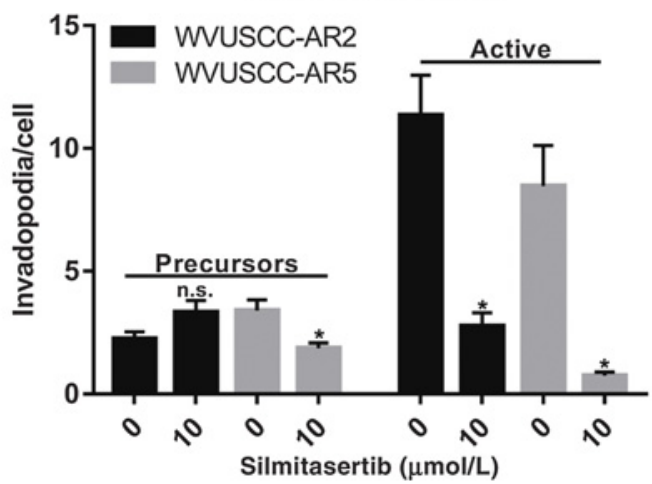

Figure 6.

Silmitasertib inhibits invadopodia function in HNSCC PDX cells. A, Establishment of HNSCC PDX tumors. Hematoxylin and eosin stained patient tumor tissue and PDXs. Patient tumors were from the alveolar ridge (AR). Scale bar represents $200 \mu \mathrm{m}$. B, Invadopodia formation in HNSCC PDX cells. Representative confocal images of WVUSCC-AR2 (AR2) and WVUSCC-AR5 (AR5) PDX cell lines. Cells were plated on OG-488 gelatin coverslips for 24 hours and labeled with an anticortactin antibody and rhodamine phalloidin (Actin). Gelatin is pseudo-colored white. Scale bar represents $20 \mu \mathrm{m}$. $10 \times$ representative phase contrast images (Phase) of each line are shown on the right. Scale bar represents $100 \mu \mathrm{m}$. C, Confocal images of WVUSCC-AR2 and WVUSCC-AR5 cells plated on OG-488 gelatin coverslips, allowed to attach for 1 hour, then treated with vehicle (DMSO) or $10 \mu \mathrm{mol} / \mathrm{L}$ Silmitasertib for 24 hours. Cells were labeled with an anti-cortactin antibody and rhodamine phalloidin (Actin). Gelatin is pseudo-colored white. Scale bar represents $20 \mu \mathrm{m}$. D, CK2 $\alpha$ inhibition decreases invadopodia-mediated ECM degradation in PDX-derived cell lines. Quantification of matrix degradation area per cell area for WVUSCC-AR2 and WVUSCC-AR5 PDX cell lines treated with the indicated Silmitasertib concentrations. Degradation data were normalized to DMSO-treated cells for each cell line. E, CK2 $\alpha$ inhibition decreases invadopodia numbers in PDX-derived cell lines. Determination of amount of invadopodia precursors (left) and active invadopodia (right) in WVUSCC-AR2 and WVUSCC-AR5 cells. Data represent the mean + SEM. of $n \geq 100$ cells analyzed from at least three independent experiments. *, $P<0.05$, Welch $t$ test vs. DMSO for each cell line.

binding sites for scaffolding platforms that recruit N-WASp and WAVE2, ensuring that activation of Arp $2 / 3$ is maintained throughout the invadopodia cycle (6). Cortactin also recruits the F-actin severing protein cofilin to invadopodia, providing the necessary machinery for cyclic actin network breakdown and regrowth during invadopod extension (16). In addition to these important C-terminal functions, the ability of the NTA region to directly bind Arp2/3 is also essential for invadopodia formation and ECM degradation, presumably through direct Arp2/3 activation and prolonged stabilization of Arp2/3-F-actin networks $(10,16)$.

Regarding the ability of the cortactin NTA to bind Arp2/3, this study reveals two distinct findings. First, that T24 is required for Arp2/3 binding, because mutation of this residue ablates (A) 
A
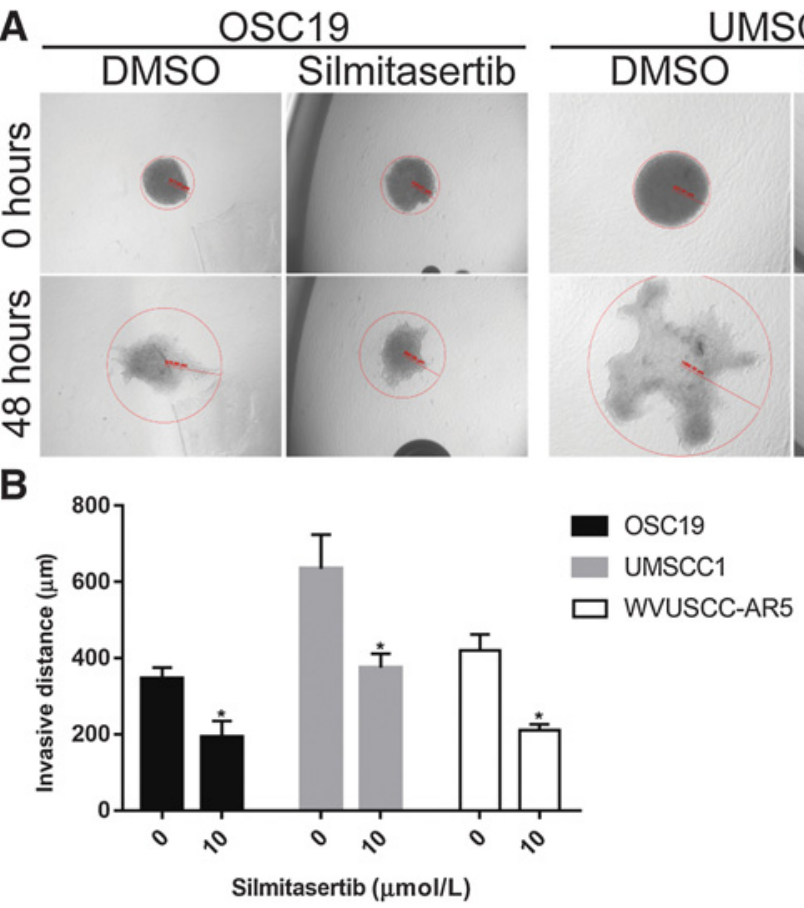

D
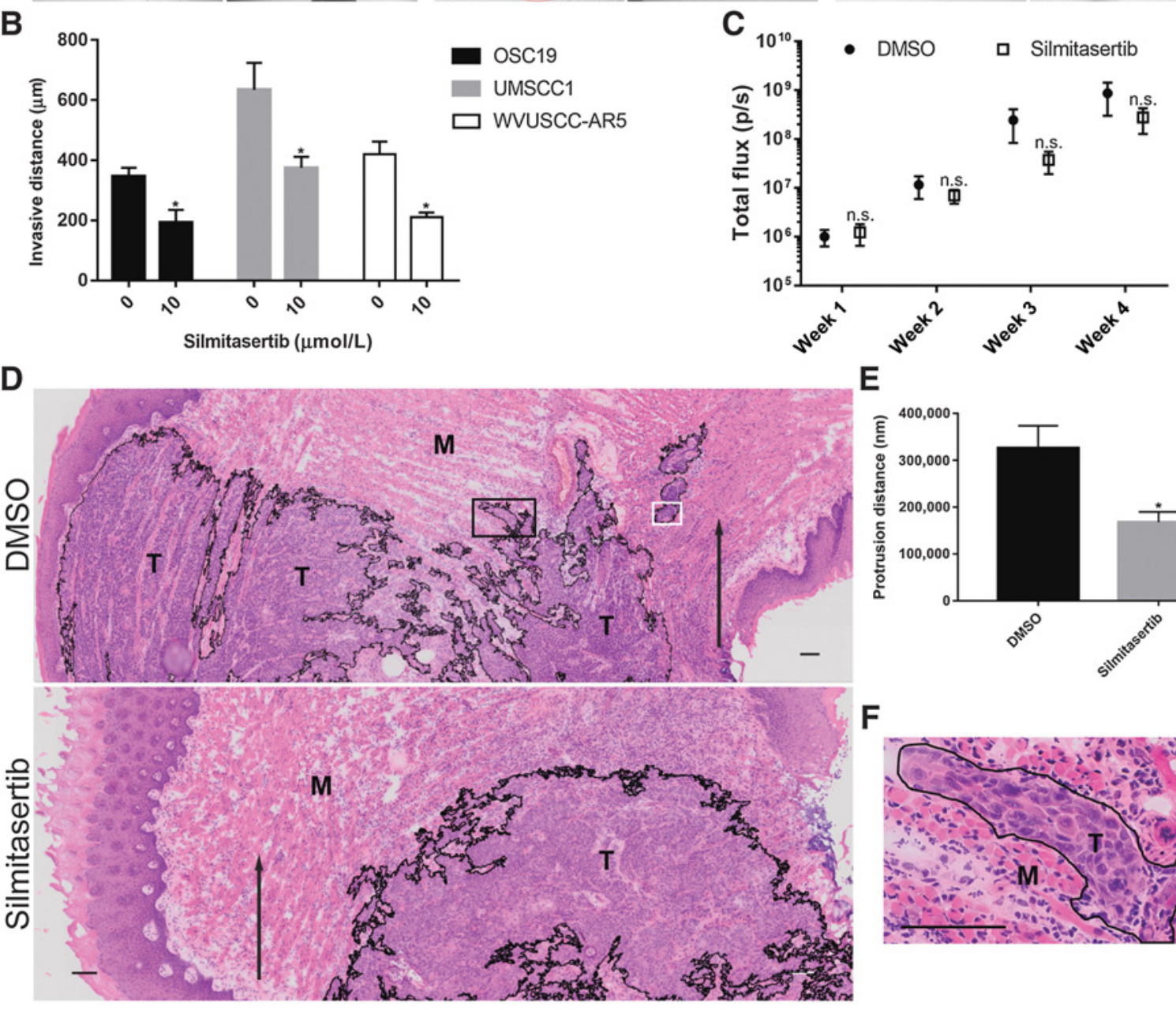

E

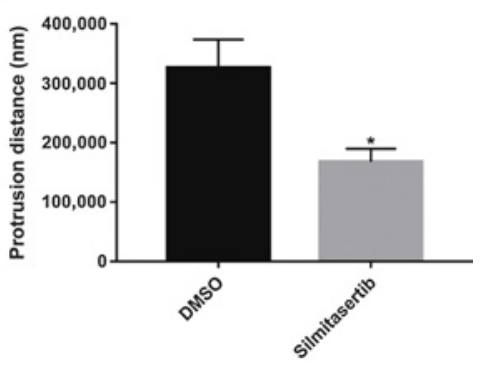

F
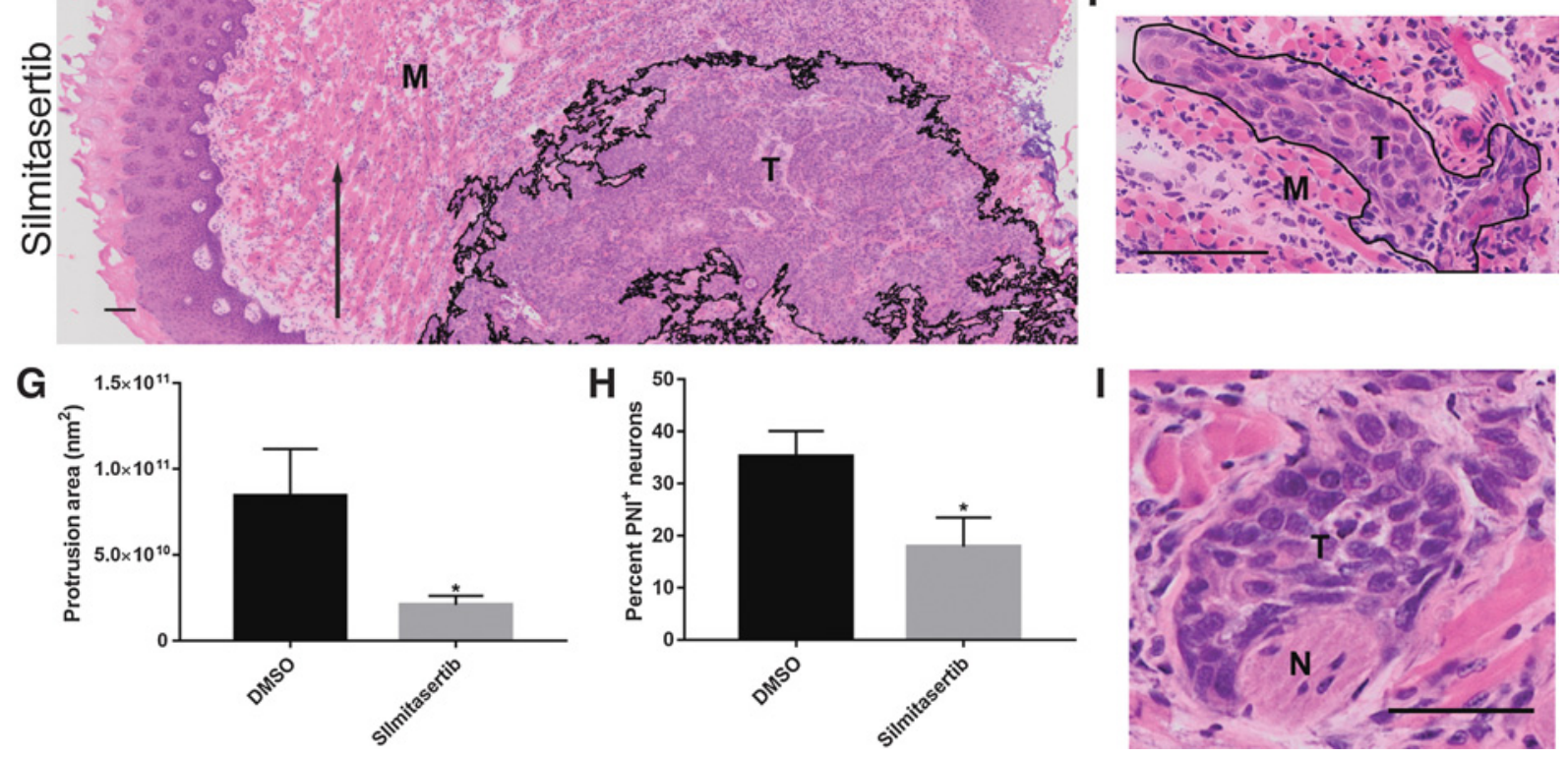
or reduces (D) the association of cortactin with Arp2/3 in coimmunoprecipitation assays. T24 is two residues C-terminal to the well-defined DDW Arp2/3 interaction motif that is conserved in other Arp2/3 NPFs (6). This places T24 in close proximity to contribute to Arp2/3 binding. Although the crystal structure of the cortactin NTA bound to Arp2/3 complex has not been reported, previous chemical crosslinking and three-dimensional reconstruction studies indicate that the NTA region primarily binds the Arp3 subunit in the complex, with the most N-terminal residues spanning the Arp2-Arp3 interface to contact the Arp2 subunit (Visual Overview; refs. 9, 34). Thus, T24 may contribute essential hydrogen bonding with recipient polar residues near the basic side chains on Arp3 involved in electrostatic binding to the acidic NTA residues. This concept is supported by the requirement of W22 in the DDW motif for Arp2/3 binding, along with the lack of an equivalent threonine in the VCA region of WASp proteins $(16,34,35)$. Although T24A cortactin appears incapable of binding Arp2/3, it can activate Arp2/3 actin nucleation in conditions where $\triangle \mathrm{DDW}$ cortactin remains inactive (Fig. 1D). This may be due to T24 having a lower affinity for Arp3 than the DDW region and/or the high cortactin molar levels required to stimulate NPF activity in vitro. Nonetheless, the requirement for cortactin T24 in invadopodia-mediated ECM degradation supports an essential biological role for this residue in a nonphosphorylated context.

Second, our study shows that T24 phosphorylation serves to negatively regulate cortactin binding to and activation of Arp2/3 complex (Fig. 3B-D). Addition of the phosphate group to T24 likely imparts a steric and electrostatic disruption, preventing the DDW and other interacting residues in the NTA from initiating and/or maintaining binding to Arp3. Steric interference may be the predominant mechanism, since T24D cortactin can bind Arp2/3 at reduced levels (Fig. 1B), indicating that negative charge alone is insufficient to completely prevent Arp2/3 association. Phosphorylation of T24 therefore serves to block the ability of cortactin to bind Arp2/3, similar to the regulation of the Arp2/3-Factin regulatory proteins coronin $1 \mathrm{~B}$ and cofilin $(46,47)$. Although we did not determine if phosphorylation of T24 serves to release cortactin from existing Arp2/3-F-actin branchpoints, phosphorylation of Arp2/3-bound cortactin T24 may be an additional mechanism to dissociate cortactin from these regions. Such a mechanism could function in facilitating breakdown and recycling of invadopodia Arp2/3-F-actin networks.

The ability of CK2 $\alpha$ to phosphorylate cortactin T24 supports a wider role for this kinase in actin regulation. Previous work identified T2 4 as a putative but unconfirmed CK2 $\alpha$ phosphorylation site in the NTA region of myeloid-cell specific cortactin homolog HS1 (21). In addition, CK2 $\alpha$ phosphorylates two adjacent sites in the A domain of WASp family proteins (22-25). These studies report conflicting results on Arp2/3 activity, with phosphorylation of WASp enhancing and in N-WASp and WAVE2 inhibiting VCA-stimulated Arp2/3-mediated actin polymerization. Our results with CK2 $\alpha$-phosphorylated N-WASp VCA domain also shows an inhibitory effect on Arp2/3 activation (Fig. 3C), suggesting that CK2 $\alpha$ effects may be differential to specific WASp family proteins.

Cortactin T24A and T24D expression in KD HNSCC lines impairs active invadopodia formation and subsequent ECM degradation (Fig. 2A-C; Supplementary Fig. S1A and S1C), supporting in vitro evidence that T24 phosphorylation prevents Arp2/3 binding and activation necessary for invadopodia activity. The importance of CK2 $\alpha$-mediated cortactin T24 phosphorylation is further supported by the inability of Silmitasertib to further suppress ECM degradation in cortactin T24A or T24D expressing cells (Fig. 5D and E). CK2 $\alpha$-specific knockdown or pharmacologic inhibition in cells would therefore be expected to reduce phosphorylation of cortactin and WASp family proteins, resulting in net increased Arp $2 / 3$ activity as observed from in vitro studies. The fact that CK $2 \alpha$ knockdown or kinase blockade also impairs invadopodia formation and function is paradoxical, but can be explained by cyclic CK2 $\alpha$ phosphorylation and dephosphorylation to regulate cortactin binding to and activation of Arp $2 / 3$ in invadopodia during maturation and elongation. Dephosphorylation of cortactin T24 and WASp NPFs by unknown phosphatases would enable these NPFs to promote Arp2/3 activation, actin polymerization, and invadopodia activity. Following network breakdown during actin turnover, CK2 $\alpha$ phosphorylation would return NPFs to the inactive state to await the next cycle of actin assembly. Although similar cyclic regulation of Src and cofilin has been shown to be essential for invadopodia function

Figure 7.

Silmitasertib impairs collective invasion in HNSCC. A, Representative phase contrast microscopy images of OSC19, UMSCC1, and WVUSCC-AR5 tumor cell spheroids embedded in collagen I ( 0 hour) then incubated in complete media containing $10 \mu \mathrm{mol} / \mathrm{L}$ Silmitasertib or vehicle (DMSO) for 48 hours. Red circles indicate the maximum radial distance at indicated time points. Scale bar represents $100 \mu \mathrm{m}$. B, Quantitation of maximal collective invasive distance of each cell line at 48 hours with vehicle ( 0 ) or Silmitasertib $(10 \mu \mathrm{mol} / \mathrm{L})$. Data represent the mean $+\mathrm{SEM}$ of $n \geq 10$ spheroids per cell line and condition analyzed from at least three independent experiments. ${ }^{*}, P<0.05$, Welch or Student $t$ test vs. DMSO. C, Effect of Silmitasertib on HNSCC orthotopic tumor growth. Bioluminescent monitoring of UMSCCl cells orthotopically injected into tongues of NSG mice. Tumors were allowed to establish for 1 week prior to administration of Silmitasertib or equal volume vehicle (DMSO). Data represent mean \pm SEM from two independent experiments. DMSO, $n=9$ mice; Silmitasertib, $n=7$ mice. n.s., not significant; Student $t$ test vs. DMSO. D, Silmitasertib inhibits orthotopic HNSCC invasion. Representative hematoxylin and eosin stained invasive front of orthotopic UMSCC1 tongue tumors from mice receiving Silmitasertib or vehicle (DMSO) for 3 weeks. Black lines show tumor-encompassing RIO borders determined by software analysis. Black box in DMSO tumor indicates the invasive protrusion shown in F. White box in DMSO tumor denotes region containing perineural invasion (PNI) shown in I. Black arrow indicates direction of invasion toward tongue base. M, skeletal muscle; T, tumor. Scale bar represents $100 \mu \mathrm{m}$. E, Invasive distance of tumor protrusions from mice treated with Silmitasertib or vehicle (DMSO). Data represent the mean + SEM of two serial sections from $N=4$ tumors; $n=34$ protrusions per condition. ${ }^{*}, P<0.05$, Student $t$ test vs. DMSO. F, Representative image of an invasive protrusion from vehicle-treated mice shown in the black box in $\mathbf{D}$. The protrusive region was traced in black to denote the tumor protrusion from surrounding muscle-containing stroma. M, skeletal muscle; T, tumor protrusion. Scale bar represents $100 \mu \mathrm{m}$. G, Area of invasive protrusions from mice treated with Silmitasertib or vehicle (DMSO). Data represent the mean + SEM of two serial sections from $N=4$ tumors; $n=34$ protrusions per condition. ${ }^{*}, P<0.05$, Welch $t$ test vs. DMSO. H, Percentage of lingual nerves displaying PNI in tumors from mice treated with Silmitasertib or vehicle (DMSO). Data represent the mean + SEM of two serial sections from $N=4$ tumors; $n \geq$ 60 nerves per condition. ${ }^{*}, P<0.05$, Student $t$ test vs. DMSO. I, Representative image of PNI shown in the white box in the DMSO-treated mouse in $\mathbf{D}$. N, nerve; T, tumor cells. Scale bar represents $50 \mu \mathrm{m}$. 
and provides support for this model $(38,46)$, confirmation of T24 phosphorylation within invadopodia leading to altered actin dynamics along with identification of the T24 phosphatase are necessary to fully confirm this proposed mechanism.

Locoregional control of HNSCC dissemination is problematic, where perineural invasion and invasive metastatic spread to cervical lymph nodes accelerates patient decline $(44,48,49)$. Cortactin and CK2 $\alpha$ expression are elevated in HNSCC and individually correspond with poorer patient outcomes $(7,20)$. CK2 knockdown and Silmitasertib treatment in HNSCC cells has antiproliferative and antimetastatic properties in vitro and in mice, providing support for the utility of Silmitasertib in HNSCC (20). The anti-invasive effect of Silmitasertib shown here is likely due in part to disruption of the invadopodia actin assembly through combined inhibition of multiple Arp2/3 NPFs. We note that T24 cortactin mutants, CK2 $\alpha$ knockdown, and Silmitasertib treatment in HNSCC cells do not completely abolish invadopodia formation, degradation activity or invasion. Activation of invadopodial Arp2/3 NPFs through alternative scaffolding or lipid-based signaling pathways that can bypass CK2 inhibition can account for this residual activity (1). However, our results indicate that CK2 $\alpha$ inhibition by a single agent does significantly impair collective HNSCC invasion. Given the current paucity of treatment options for invasive HNSCC, combining Silmitasertib with additional drugs known to impair invadopodia function by blocking additional invadopodia and motility pathways (8) has the potential to provide novel treatment options for controlling invasive spread of late-stage HNSCC harboring elevated cortactin and/or CK2 expression.

\section{Disclosure of Potential Conflicts of Interest}

S.A. Weed reports receiving a commercial research grant from Gilead Sciences. No potential conflicts of interest were disclosed by the other authors.

\section{Disclaimer}

This laboratory was supported in part by a grant from Gilead Sciences during the period when the work was conducted. Gilead grant funds were not used to support any aspect of the project reported in this manuscript. Gilead Sciences had no input on the findings disclosed herein.

\section{Authors' Contributions}

Conception and design: S.M. Markwell, E.T. Interval, S.A. Weed

Development of methodology: S.M. Markwell, E.T. Interval, D.A. Schafer

Acquisition of data (provided animals, acquired and managed patients, provided facilities, etc.): S.M. Markwell, A.G. Ammer, E.T. Interval, J.L. Allen, B.W. Papenberg, R.A. Hames, J.E. Castaño, D.A. Schafer, S.A. Weed Analysis and interpretation of data (e.g., statistical analysis, biostatistics, computational analysis): S.M. Markwell, A.G. Ammer, E.T. Interval, B.W. Papenberg, S.A. Weed

Writing, review, and/or revision of the manuscript: S.M. Markwell, E.T. Interval, S.A. Weed

Study supervision: S.A. Weed

Other (figure design): B.W. Papenberg

Other (I provided reagents for the actin assembly assays and guidance to Dr. Markwell as he performed the assays in my lab.): D.A. Schafer

\section{Acknowledgments}

The authors thank Jianjiong Gao (University of Missouri) for sequence prediction analysis. The assistance of the West Virginia University Microscope Imaging Facility, Animal Models and Imaging Facility, and Biochemistry Protein Core are gratefully acknowledged.

This work was supported by pilot grants from NIH U54GM104942, P20RR016440, and P20GM103434 (to S.M. Markwell, A.G. Ammer, J.L. Allen B.W. Papenberg, R.A. Hames, and S.A. Weed). Additional support was provided by the West Virginia University Department of Neurobiology and Anatomy (to S.M. Markwell, S.A. Weed), Department of Biochemistry (to S.M. Markwell, S.A. Weed), Department of Otolaryngology, Head and Neck Surgery (to E.T. Interval, J.E. Castaño), and the Dorothy D. Radford Endowed Fund of the West Virginia University Cancer Institute (to S.A. Weed). Instrumentation support was provided by NIH grants U54GM104942, P20RR016440, P30RR032138/GM103488, P20GM103434, and P20RR016477. The content is solely the responsibility of the authors and does not necessarily represent the official views of the NIH.

The costs of publication of this article were defrayed in part by the payment of page charges. This article must therefore be hereby marked advertisement in accordance with 18 U.S.C. Section 1734 solely to indicate this fact.

Received April 20, 2018; revised September 18, 2018; accepted December 12 , 2018; published first January 4, 2019.

\section{References}

1. Eddy RJ, Weidmann MD, Sharma VP, Condeelis JS. Tumor cell invadopodia: invasive protrusions that orchestrate metastasis. Trends Cell Biol 2017; 27:595-607.

2. Markwell S, Weed S. Tumor and stromal-based contributions to head and neck squamous cell carcinoma invasion. Cancers (Basel) 2015;7:382-406.

3. Bowden ET, Barth M, Thomas D, Glazer RI, Mueller SC. An invasion-related complex of cortactin, paxillin and PKCmu associates with invadopodia at sites of extracellular matrix degradation. Oncogene 1999;18:4440-9.

4. Branch KM, Hoshino D, Weaver AM. Adhesion rings surround invadopodia and promote maturation. Biol Open 2012;1:711-22.

5. Schnoor M, Stradal TE, Rottner K. Cortactin: cell functions of a multifaceted actin-binding protein. Trends Cell Biol 2018;28:79-98.

6. Yin M, Ma W, An L. Cortactin in cancer cell migration and invasion. Oncotarget 2017;8:88232-43.

7. MacGrath SM, Koleske AJ. Cortactin in cell migration and cancer at a glance. J Cell Sci 2012;125:1621-6.

8. Meirson T, Gil-Henn H. Targeting invadopodia for blocking breast cancer metastasis. Drug Resist Updat 2018;39:1-17.

9. Xu XP, Rouiller I, Slaughter BD, Egile C, Kim E, Unruh JR, et al. Threedimensional reconstructions of Arp2/3 complex with bound nucleation promoting factors. EMBO J 2012;31:236-47.

10. Weaver AM, Karginov AV, Kinley AW, Weed SA, Li Y, Parsons JT, et al. Cortactin promotes and stabilizes Arp2/3-induced actin filament network formation. Curr Biol 2001;11:370-4.

11. Helgeson LA, Prendergast JG, Wagner AR, Rodnick-Smith M, Nolen BJ. Interactions with actin monomers, actin filaments and Arp2/3 complex define the roles of WASP family proteins and cortactin in coordinately regulating branched actin networks. J Biol Chem 2014;289:1-26.

12. Weed SA, Karginov AV, Schafer DA, Weaver AM, Kinley AW, Cooper JA et al. Cortactin localization to sites of actin assembly in lamellipodia requires interactions with F-actin and the Arp2/3 complex. J Cell Biol 2000;151:29-40.

13. Weaver AM, Young ME, Lee WL, Cooper JA. Integration of signals to the Arp2/3 complex. Curr Opin Cell Biol 2003;15:23-30.

14. Blanchoin L, Amann KJ, Higgs HN, Marchand J, Kaiser DA, Pollard TD. Direct observation of dendritic actin filament networks nucleated by Arp2/ 3 complex and WASP / Scar proteins. Nature 2000;404:1007-11.

15. Martinez-Quiles N, Ho HY, Kirschner MW, Ramesh N, Geha RS. Erk / Src phosphorylation of cortactin acts as a switch on-switch off mechanism that controls its ability to activate N-WASP. Mol Cell Biol 2004;24:5269-80.

16. Oser M, Yamaguchi H, Mader CC, Bravo-Cordero JJ, Arias M, Chen X, et al. Cortactin regulates cofilin and N-WASp activities to control the stages of invadopodium assembly and maturation. J Cell Biol 2009;186 571-87.

17. Mader CC, Oser M, Magalhaes MA, Bravo-Cordero JJ, Condeelis J, Koleske AJ, et al. An EGFR-Src-Arg-Cortactin pathway mediates functional maturation of invadopodia and breast cancer cell invasion. Cancer Res 2011;71: $1730-41$. 
18. Ayala I, Baldassarre M, Giacchetti G, Caldieri G, Tetè S, Luini A, et al Multiple regulatory inputs converge on cortactin to control invadopodia biogenesis and extracellular matrix degradation. J Cell Sci 2008;121: 369-78.

19. Martin KH, Jeffery ED, Grigera PR, Shabanowitz J, Hunt DF, Parsons JT. Cortactin phosphorylation sites mapped by mass spectrometry. J Cell Sci 2006;119:2851-3

20. Chua MMJ, Ortega CE, Sheikh A, Lee M, Abdul-Rassoul H, Hartshorn KL, et al. CK2 in cancer: cellular and biochemical mechanisms and potential therapeutic target. Pharmaceuticals (Basel) 2017;10:piiE18.

21. Ruzzene M, Brunati AM, Sarno S, Marin O, Donella-Deana A, Pinna LA. Ser/Thr phosphorylation of hematopoietic specific protein 1 (HS1): implication of protein kinase CK2. Eur J Biochem 2000;267:3065-72.

22. Mendoza MC. Phosphoregulation of the WAVE regulatory complex and signal integration. Semin Cell Dev Biol 2013;24:272-9.

23. Pocha SM, Cory GO. WAVE2 is regulated by multiple phosphorylation events within its VCA domain. Cell Motil Cytoskeleton 2009;66: 36-47.

24. Cory GOC, Cramer R, Blanchoin L, Ridley AJ. Phosphorylation of the WASP-VCA domain increases its affinity for the Arp2/3 complex and enhances actin polymerization by WASP. Mol Cell 2003;11:1229-39.

25. Galovic M, Xu D, Areces LB, van der Kammen R, Innocenti M. Interplay between N-WASP and CK2 optimizes clathrin-mediated endocytosis of EGFR. J Cell Sci 2011;124:2001-12.

26. Hayes KE, Walk EL, Ammer AG, Kelley LC, Martin KH, Weed SA. Ableson kinases negatively regulate invadopodia function and invasion in head and neck squamous cell carcinoma by inhibiting an HB-EGF autocrine loop. Oncogene 2013;32:4766-77.

27. Ammer AG, Kelley LC, Hayes KE, Evans J V, Lopez-skinner A, Martin KH, et al. Saracatinib impairs head and neck squamous cell carcinoma invasion by disruption invadopodia function. J Cancer Sci Ther 2009;1:52-61.

28. Head JA, Jiang D, Li M, Zorn LJ, Schaefer EM, Parsons JT, et al. Cortactin tyrosine phosphorylation requires Rac1 activity and association with the cortical actin cytoskeleton. Mol Biol Cell 2003;14:3216-29.

29. Martin KH, Hayes KE, Walk EL, Ammer AG, Markwell SM, Weed SA Quantitative measurement of invadopodia-mediated extracellular matrix proteolysis in single and multicellular contexts. J Vis Exp 2012;e4119.

30. Kelley LC, Weed SA. Cortactin is a substrate of activated Cdc42-associated kinase 1 (ACK1) during ligand-induced epidermal growth factor receptor downregulation. PLoS One 2012;7:e44363.

31. Mooren OL, Kotova TI, Moore AJ, Schafer DA. Dynamin2 GTPase and cortactin remodel actin filaments. J Biol Chem 2009;284:23995-4005.

32. Basu D, Nguyen T-TK, Montone KT, Zhang G, Wang L-P, Diehl JA, et al Evidence for mesenchymal-like sub-populations within squamous cell carcinomas possessing chemoresistance and phenotypic plasticity. Oncogene 2010;29:4170-82.

33. Gatesman Ammer A, Hayes KE, Martin KH, Zhang L, Spirou GA, Weed SA. Multi-photon imaging of tumor cell invasion in an orthotopic mouse model of oral squamous cell carcinoma. J Vis Exp 2011;pii: 2941.
34. Weaver AM, Heuser JE, Karginov AV, Lee W, Parsons JT, Cooper JA Interaction of cortactin and N-WASp with Arp2/3 complex. Curr Biol 2002;12:1270-8.

35. Uruno T, Liu J, Zhang P, Fan Y, Egile C, Li R, et al. Activation of Arp2/3 complex-mediated actin polymerization by cortactin. Nat Cell Biol 2001;3: 259-66.

36. Clark ES, Whigham AS, Yarbrough WG, Weaver AM. Cortactin is an essential regulator of matrix metalloproteinase secretion and extracellular matrix degradation in invadopodia. Cancer Res 2007;67:4227-35.

37. Siton O, Ideses Y, Albeck S, Unger T, Bershadsky AD, Gov NS, et al. Cortactin releases the brakes in actin- based motility by enhancing WASP-VCA detachment from Arp2/3 branches. Curr Biol 2011;21:2092-7.

38. Kelley LC, Ammer AG, Hayes KE, Martin KH, Machida K, Jia L, et al. Oncogenic Src requires a wild-type counterpart to regulate invadopodia maturation. J Cell Sci 2010;123:3923-32.

39. Vadlamudi RK, Li F, Barnes CJ, Bagheri-Yarmand R, Kumar R. p41-Arc subunit of human Arp2/3 complex is a p21-activated kinase-1-interacting substrate. EMBO Rep 2004;5:154-60.

40. Kazazian K, Go C, Wu H, Brashavitskaya O, Xu R, Dennis JW, et al. Plk4 promotes cancer invasion and metastasis through Arp2/3 complex regulation of the actin cytoskeleton. Cancer Res 2017;77:434-47.

41. LeClaire LL, Rana M, Baumgartner $M$, Barber DL. The Nck-interacting kinase NIK increases Arp2/3 complex activity by phosphorylating the Arp2 subunit. J Cell Biol 2015;208:161-70.

42. Siddiqui-Jain A, Drygin D, Streiner N, Chua P, Pierre F, O'Brien SE, et al. CX 4945, an orally bioavailable selective inhibitor of protein kinase CK2, inhibits prosurvival and angiogenic signaling and exhibits antitumor efficacy. Cancer Res 2010;70:10288-98.

43. Zhao M, Sano D, Pickering CR, Jasser SA, Henderson YC, Clayman GL, et al Assembly and initial characterization of a panel of 85 genomically validated cell lines from diverse head and neck tumor sites. Clin Cancer Res 2011;17:7248-64.

44. Kurtz KA, Hoffman HT, Bridget Zimmerman M, Robinson RA, Carver LA. Perineural and vascular invasion in oral cavity squamous carcinoma increased incidence on re-review of slides and by using immunohistochemical enhancement. Arch Pathol Lab Med 2005;129:354-9.

45. Kelley LC, Hayes KE, Ammer AG, Martin KH, Weed SA. Cortactin phosphorylated by ERK1/2 localizes to sites of dynamic actin regulation and is required for carcinoma lamellipodia persistence. PLoS One 2010;5:e13847.

46. Oser $\mathrm{M}$, Condeelis J. The cofilin activity cycle in lamellipodia and invadopodia. J Cell Biochem 2009;108:1252-62.

47. Cai L, Holoweckyj N, Schaller MD, Bear JE. Phosphorylation of coronin 1B by protein kinase $\mathrm{C}$ regulates interaction with Arp $2 / 3$ and cell motility. J Biol Chem 2005;280:31913-23.

48. Xing Y, Zhang J, Lin H, Gold KA, Sturgis EM, Garden AS, et al. Relation between the level of lymph node metastasis and survival in locally advanced head and neck squamous cell carcinoma. Cancer 2016;122:534-45.

49. Fagan JJ, Collins B, Barnes L, D'Amico F, Myers EN, Johnson JT. Perineural invasion in squamous cell carcinoma of the head and neck. Arch Otolaryngol Neck Surg 1998;124:637. 


\section{Molecular Cancer Research}

\section{Cortactin Phosphorylation by Casein Kinase 2 Regulates Actin-Related Protein 2/3 Complex Activity, Invadopodia Function, and Tumor Cell Invasion}

Steven M. Markwell, Amanda G. Ammer, Erik T. Interval, et al.

Mol Cancer Res 2019;17:987-1001. Published OnlineFirst January 4, 2019.

\section{Updated version Access the most recent version of this article at: doi:10.1158/1541-7786.MCR-18-0391}

Supplementary Access the most recent supplemental material at:

Material http://mcr.aacrjournals.org/content/suppl/2019/01/04/1541-7786.MCR-18-0391.DC1

Visual A diagrammatic summary of the major findings and biological implications:

Overview http://mcr.aacrjournals.org/content/17/4/987/F1.large.jpg

Cited articles This article cites 47 articles, 21 of which you can access for free at:

http://mcr.aacrjournals.org/content/17/4/987.full\#ref-list-1

E-mail alerts

Reprints and

Subscriptions

Permissions
Sign up to receive free email-alerts related to this article or journal.

To order reprints of this article or to subscribe to the journal, contact the AACR Publications Department at pubs@aacr.org.

To request permission to re-use all or part of this article, use this link http://mcr.aacrjournals.org/content/17/4/987.

Click on "Request Permissions" which will take you to the Copyright Clearance Center's (CCC)

Rightslink site. 


\section{When the Smoke Clears $\mathrm{m}^{6} \mathrm{~A}$ from a $\mathrm{Y}$ Chromosome- Linked IncRNA, Men Get an Increased Risk of Cancer}

A. Rouf Banday', Brenen W. Papenberg ${ }^{2}$, and Ludmila Prokunina-Olsson ${ }^{1}$

Long noncoding RNAs (lncRNA) have been implicated in many diseases, including cancer. Although these diseaseassociated effects have been mostly attributed to the ability of $\operatorname{lncRNAs}$ to function as regulatory noncoding transcripts, there is growing evidence that lncRNAs may also encode functional micropeptides. In the current issue of Cancer Research, $\mathrm{Wu}$ and colleagues report a micropeptide encoded by a Y chromosome-

Long noncoding RNAs (lncRNA) are generally defined as long ( $>200$ nucleotides) transcripts that do not encode proteins. LncRNAs include some well-known regulatory transcripts that affect cancer initiation and progression, such as MALAT1 (metastasis-associated lung adenocarcinoma transcript 1), HOTAIR (HOX transcript antisense intergenic RNA), UCA1 (urothelial carcinoma associated 1), $B C Y R N 1$ (brain cytoplasmic RNA 1), and HULC (highly upregulated in liver cancer; ref. 1). Despite the "noncoding" designation, multiple short open reading frames (ORF) for putative micropeptides can usually be predicted within lncRNAs. An annotation of a 53-amino acid peptide, HOX-AS3, which inhibits tumorigenesis in the colon by metabolic reprogramming, provides one of a few examples demonstrating functional properties of micropeptides rather than their encoding lncRNAs (2). However, due to limited understanding of their functions and lack of high-throughput methods for their annotation, these putative IncRNA-encoded micropeptides are mostly overlooked.

In the current issue of Cancer Research, $\mathrm{Wu}$ and colleagues (3) present their discovery of a 21 -amino acid micropeptide (designated as YY1BM) encoded by a Y chromosome-linked lncRNA, LINC00278. They demonstrated the role of YY1BM in tumorigenesis and cancer survival through its interaction with smoking exposure in male esophageal squamous cell carcinoma (ESCC). ESCC is one of the deadliest cancers in the world, with average 5-year survival rates remaining under $20 \%$. Tobacco smoking is considered a major environmental risk factor for ESCC. Men are four times more likely to develop ESCC than women, which might be partially explained by the differences in smoking rates reported as $52.9 \%$ and $2.4 \%$ in Chinese men and women, respectively (4), with comparable ratios in other populations as well. Male-specific factors, both hormonal and genetic, such as those encoded by the $\mathrm{Y}$ chromosome, might be contributing to

'Laboratory of Translational Genomics, Division of Cancer Epidemiology and Genetics, $\mathrm{NCl}$, NIH, Bethesda, Maryland. ${ }^{2}$ Program in Cancer Cell Biology, Department of Biochemistry, West Virginia University, Morgantown, West Virginia.

Corresponding Author: Ludmila Prokunina-Olsson, NCl, NIH, 9615 Medical Center Dr, Bethesda, MD 20892-9776. Phone: 240-760-6531; Fax: 240-5414442; E-mail: prokuninal@mail.nih.gov

Cancer Res 2020;80:2718-9

doi: 10.1158/0008-5472.CAN-20-0961

(C)2020 American Association for Cancer Research. linked lncRNA that may explain the higher incidence of esophageal cancer in male smokers. Furthermore, this report provides broader insights related to the molecular epidemiology of maledominant and smoking-driven cancers and may also help explain some cancer-related associations with mosaic Y chromosome loss.

See related article by $\mathrm{Wu}$ et al., p. 2790 this complex disease, in interaction with environmental exposures This combination of risk factors is not specific to ESCC and might also be relevant to other male-dominant and smoking-driven cancers, such as of the head and neck, bladder, lung, and colon. However, molecular mechanisms that can explain this gender bias in several common cancers, especially in smokers, remain largely unclear. In their study, $\mathrm{Wu}$ and colleagues hypothesized that some of these answers might be literally encoded within the $\mathrm{Y}$ chromosome and chose to focus on lncRNAs.

The authors found that the expression of three Y chromosomeencoded lncRNAs was downregulated in esophageal tumors compared to adjacent normal tissues; of those, only downregulation of LINC00278 was validated in an additional set of samples. Next, they experimentally demonstrated that one of the four ORFs predicted within this $\operatorname{lncRNA}$ encoded a 21 -amino acid $(2.12 \mathrm{kDa})$ micropeptide. Through rigorous in vitro and in vivo work, they demonstrated how downregulation of LINC00278, and therefore the reduced production of the corresponding micropeptide, could lead to ESCC in male smokers. Specifically, they showed that the micropeptide could bind the YY1 transcription factor, thereby blocking its interaction with the androgen receptor (AR). On the basis of these findings, the micropeptide was designated as the YY1-binding micropeptide (YY1BM). Low YY1BM expression resulted in reduced apoptosis in ESCC xenografts and tissues by decreasing the AR-regulated eEF2K expression, which controls protein synthesis. This effect was particularly noticeable under cellular stress conditions of severe nutrient deprivation, which might mimic the microenvironment of rapidly growing tumor cells. Thus, YY1BM might be inhibiting tumorigenesis by mediating cross-talk between pathways involving AR signaling, protein synthesis, and cell metabolism. These findings could also justify using AR-targeted therapies for men with ESCC and other cancers to be identified affected by decreased YY1BM expression.

Smoking is the major environmental risk factor for many cancer types, but the molecular mechanisms of smoking exposure are still only partially understood. It is also unclear whether smoking has different cancer-related effects in men and women, and if so, why? Wu and colleagues provide the most unexpected answer to this question. Dynamic regulation of transcriptional outcomes through the $N^{6}$. methyladenosine $\left(\mathrm{m}^{6} \mathrm{~A}\right)$ posttranscriptional RNA modification emerged as a mechanism of cellular adaptation to physiological and environmental conditions (5). This process is regulated by a combination of proteins with specific functions to add/write (methyltransferases), erase (demethylases), or read $\mathrm{m}^{6} \mathrm{~A}$ on various transcripts. By 
exposing cells to cigarette smoke condensate, the authors identified a complex of METTL3/14 and WATP as a writer, ALKBH5 as an eraser and YTHDF1 as a reader, all affecting translation of LINC00278 into YY1BM. Of these proteins critical for $\mathrm{m}^{6} \mathrm{~A}$ function, only expression of ALKBH5 (an eraser) was increased by smoking exposure, through its promoter hypomethylation. Thus, it appears that smoking exposure facilitates $\mathrm{m}^{6} \mathrm{~A}$ clearance from $\mathrm{LINC} 00278$ by activating the expression of ALKBH5 $\left(\mathrm{m}^{6} \mathrm{~A}\right.$ eraser), resulting in decreased production of YY1BM, thereby contributing to tumorigenesis. Because YY1BM can be produced only in men, women with equal smoking exposure would not have the same cancer-related molecular consequences of smoking.

The authors also linked the reduced expression of LINC00278 with decreased survival of men with ESCC, particularly in smokers. It was assumed that this association is related to the decreased production of YY1BM, given its antitumorigenic properties. However, this might not be entirely attributable to the expression or the functional effects of this lncRNA, considering several competing or overlapping mechanisms. First of all, LINC00278 is adjacent to the ZFY gene, which is one of the genes affected by the extreme downregulation of chromosome $\mathrm{Y}$ gene expression (EDY), a phenomenon associated with age and smoking in men (6). Because $Z F Y$ was affected by EDY in multiple nontumor as well as tumor tissues, mRNA expression of LINC00278 (not tested by that study) might also be affected by EDY in general and not specifically in ESCC. Focusing on lncRNAs, Wu and colleagues did not analyze any mRNA expression, including that of $Z F Y$. Thus, it is difficult to disentangle whether LINC00278 expression is the sole reason for the reported association with decreased survival, given that other genes in this region may be equally downregulated.

Downregulation of LINC00278 might also be a molecular manifestation of the age- and smoking-dependent mosaic loss of $\mathrm{Y}$ chromosome (mLOY), which affects a fraction of somatic cells already in normal tissues and is further enhanced in tumors $(7,8)$. Although both mLOY and EDY could serve as informative biomarkers, molecular mechanisms underlying cancer-related consequences of these events are still unclear. Downregulation of $Z F Y$ has been suggested to have some cancer-related functional significance, but with limited experimental evidence. On the basis of the results presented by $\mathrm{Wu}$ and colleagues, downregulation or loss of LINC00278 expression might offer at least a partial explanation of why mLOY and EDY are likely to be associated with cancer, especially in smokers. First, both these mechanisms would result in a decrease of LINC00278 expression, and thus, of YY1BM. Second, smoking-mediated clearance of $\mathrm{m}^{6} \mathrm{~A}$ from LINC00278 would further prevent the translation of this micropeptide from LINC00278, which might already be decreased through mLOY or EDY. In other words, by affecting $\mathrm{m}^{6} \mathrm{~A}$-dependent translation of

\section{References}

1. Bhan A, Soleimani M, Mandal SS. Long noncoding RNA and cancer: a new paradigm. Cancer Res 2017;77:3965-81.

2. Huang JZ, Chen M, Chen Gao XC, Zhu S, Huang H, et al. A peptide encoded by a putative IncRNA HOXB-AS3 suppresses colon cancer growth. Mol Cell 2017;68 171-84.

3. Wu S, Zhang L, Deng J, Guo B, Li F, Wang Y, et al. A novel micropeptide encoded by Y-linked LINC00278 links cigarette smoking and AR signaling in male esophageal squamous cell carcinoma. Cancer Res 2020;80:2790-803.

4. Li Q, Hsia J, Yang G. Prevalence of smoking in China in 2010. N Engl J Med 2011; 364:2469-70.

5. Chen XY, Zhang J, Zhu JS. The role of m(6)A RNA methylation in human cancer. Mol Cancer 2019;18:103. DOI: 10.1186/s12943019-1033-z.
YY1BM, smoking would likely increase cancer risk even without mLOY. However, the risk might be stronger in smokers with mLOY, due to downregulation of YY1BM by two different mechanisms.

$\mathrm{Wu}$ and colleagues also demonstrated that intratumoral treatment with recombinant YY1BM significantly reduced tumor growth and improved the ESCC survival in male xenograft mouse models. A recent study showed that another lncRNA-encoded micropeptide inhibited triple-negative breast cancer progression (9). Micropeptides are attractive as therapeutic agents due to their high specificity, potency, and low toxicity. Insulin is a well-studied example of a natural 51-amino acid minipeptide encoding an essential hormone, which is used as the main drug to treat type I diabetes. On the basis of cell membrane penetration and solubility, mini- and micropeptides might be more optimal than large proteins encoded by the bona fide protein-coding genes. Numerous micropeptides encoded by noncoding RNAs, including lncRNAs, might offer treasure troves of natural small-molecule-type peptides of possible biological significance. A recent genome-wide functional study has given a broad look at the translation of ORFs outside of canonical coding sequences (10). Among those, about 100 lncRNAencoded micropeptides were found to regulate cell growth, highlighting their potential importance in cancer and other diseases. While these studies need extensive validation, we might expect some new cancer drugs to be found among naturally existing lncRNA-encoded micropeptides.

Of multiple findings from this study of significance across several fields, we will reiterate several main points: micropeptides encoded by short lncRNA-encoded ORFs might have major cancer-related functions; these functions may be modified by environmental risk factors such as smoking and contribute to gender bias; micropeptides could be the source of naturally existing small molecules and potential anticancer therapeutic agents. The molecular mechanism presented by $\mathrm{Wu}$ and colleagues offers a plausible explanation for the higher risk of ESCC in men than women, and smoking men, specifically. This study should inspire follow-up analyses in other smoking-driven cancers affecting both sexes, but with a disproportional male:female ratio.

\section{Disclosure of Potential Conflicts of Interest}

No potential conflicts of interest were disclosed.

\section{Acknowledgments}

This work was supported by the Intramural Research Program of the NIH, CI, Division of Cancer Epidemiology and Genetics.

Received March 25, 2020; accepted March 25, 2020; published first July 1, 2020

6. Caceres A, Jene A, Esko T, Perez-Jurado LA, Gonzalez JR. Extreme down-regulation of chromosome $Y$ and cancer risk in men. J Natl Cancer Inst 2020. DOI: 10.1093/ jnci/djz232.

7. Loftfield E, Zhou W, Yeager M, Chanock SJ, Freedman ND, Machiela MJ. Mosaic Y loss is moderately associated with solid tumor risk. Cancer Res 2019;79:461-6.

8. Zhou W, Machiela MJ, Freedman ND, Rothman N, Malats N, Dagnall C, et al. Mosaic loss of chromosome $\mathrm{Y}$ is associated with common variation near TCL1A Nat Genet 2016;48:563-8.

9. Guo B, Wu S, Zhu X, Zhang L, Deng J, Li F, et al. Micropeptide CIP2A-BP encoded by LINC00665 inhibits triple-negative breast cancer progression. EMBO J 2020;39:e102190. DOI: 10.15252/embj.2019102190.

10. Chen J, Brunner AD, Cogan JZ, Nunez JK, Fields AP, Adamson B, et al. Pervasive functional translation of noncanonical human open reading frames. Science 2020;367:1140-6. 


\section{Cancer Research}

$A A C R$ 年erican Assciation

The Journal of Cancer Research (1916-1930) | The American Journal of Cancer (1931-1940)

\section{When the Smoke Clears $\mathrm{m}^{6} \mathrm{~A}$ from a $\mathrm{Y}$ Chromosome-Linked IncRNA, Men Get an Increased Risk of Cancer}

A. Rouf Banday, Brenen W. Papenberg and Ludmila Prokunina-Olsson

Cancer Res 2020;80:2718-2719.

Updated version Access the most recent version of this article at:

http://cancerres.aacrjournals.org/content/80/13/2718

Cited articles This article cites 9 articles, 4 of which you can access for free at: http://cancerres.aacrjournals.org/content/80/13/2718.full\#ref-list-1

E-mail alerts Sign up to receive free email-alerts related to this article or journal.

Reprints and To order reprints of this article or to subscribe to the journal, contact the AACR Publications Department at Subscriptions pubs@aacr.org.

Permissions To request permission to re-use all or part of this article, use this link http://cancerres.aacrjournals.org/content/80/13/2718.

Click on "Request Permissions" which will take you to the Copyright Clearance Center's (CCC) Rightslink site. 


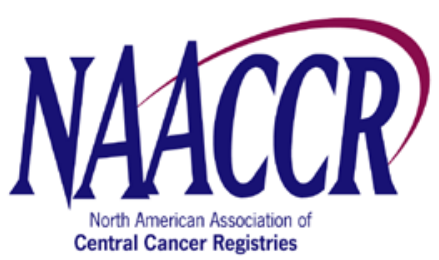

Board of Directors

Randi K. Rycroft, MSPH, CTR President

Winny Roshala, BA, CTR President-Elect

Maria J. Schymura, PhD Treasurer

Lori Swain, BA, MS Sponsoring Member Representative

Representatives-at-Large Isaac Hands, MPH Monique Hernandez, PhD Mary Jane King, MPH, CTR Angela L. W. Meisner, MPH Lorraine Shack, PhD Kevin Ward, PhD

Executive Director Betsy A. Kohler, MPH, CTR

\section{Executive Office}

2050 W. Iles Ave. Suite A Springfield, IL 62704-4194

Tel (217) 698-0800

Fax (217) 698-0188 info@naaccr.org www.naaccr.org
July 14,2020

Brenen W. Papenberg

Graduate Assistant

Mary Babb Randolph Cancer Center Room 1833

West Virginia University

Morgantown, WV 26506

Dear Mr. Papenberg:

This letter is sent to provide approval for you to use NAACCR's logo in your dissertation that will be titled "Novel Roles of Tobacco-associated Genes Underlying Disparate Survival in Appalachian Head and Neck Squamous Cell Carcinoma" which contains the results of IRB \#16-14. The use of the logo is approved for the example you provided in your 7-13-2020 email "Use of NAACCR Logo".

Please remember to cite the CiNA dataset you used. For example, the selected citation for his standard CiNA file used is: SEER ${ }^{\star}$ Stat Database: NAACCR Incidence Data - CiNA Analytic File, 1995-2014, for Expanded Races, Standard File, Weed - Appalachia Survival (which includes data from CDC's National Program of Cancer Registries (NPCR), CCCR's Provincial and Territorial Registries, and the NCl's Surveillance, Epidemiology and End Results (SEER) Registries), certified by the North American Association of Central Cancer Registries (NAACCR) as meeting high-quality incidence data standards for the specified time periods, submitted December 2016.)

Please contact me if you have any further questions.

Sincerely,

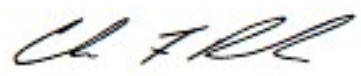

Charlie Blackburn

$\mathrm{COO}$

NAACCR, Inc. 


\section{Brenen Papenberg}

130 Mountaineer Vlg Morgantown, WV 26508 C (641) 691-6090 M bwpapenberg@gmail.com

\section{Personal Statement}

Cancer cell biologist with seven years graduate research experience studying disparate populations through secondary analysis of cancer registry data and bioinformatic interrogation of The Cancer Genome Atlas. Have also identified novel oncogenic roles of amplified and overexpressed transcripts associated with tobaccoinduced DNA damage in head and neck squamous cell carcinoma.

\section{Research Experience}

West Virginia University · Morgantown, WV

Biochemistry Department, Research Assistant · July 2014 - Present

Doctoral research projects under Scott Weed focused on identifying a novel health disparity for tobacco-correlated head and neck squamous cell carcinoma, determining tobacco-correlated gene amplifications, and identifying novel functions of cell cycle transcripts.

lowa State University of Science and Technology · Ames, IA

Microbiology Department, Laboratory Assistant · May 2012 - May 2013

Undergraduate research project under Larry Halverson focused on biofilm formation of Pseudomonas putida and its ability to colonize maize rhizospheres.

\section{Education}

West Virginia University · Morgantown, WV

Doctor of Philosophy, Cancer Cell Biology · Expected August 2020

Dissertation Title: Novel Roles of Tobacco-associated Genes Underlying Disparate Survival in Appalachian Head and Neck Squamous Cell Carcinoma

Committee: Ivan Martinez (chair), Peter Stoilov, Michael Ruppert, John Hollander, Sijin Wen

Cumulative GPA: 3.7 / 4.0

lowa State University of Science and Technology - Ames, IA

Bachelor of Science, Microbiology and Genetics · Awarded May 2013

Cumulative GPA: 3.5 / 4.0

\section{Scientific Presentations}

Oral Presentations

Papenberg, BW, Ingles, J, Feng, J, Allen, J, Markwell, S, Hames, R, Interval, E, Montague, A, Patel, R, Coad, J, Wen, S, Weed, SA. Molecular characterization of the mortality disparity in tobacco-associated Appalachian HNSCC. NAACCR Annual Conference. Pittsburgh, PA. June 2018.

Poster Presentations

Papenberg, BW, Martinez, I, Weed, SA. Oncogenic roles of overexpressed transcripts from the 11q13 amplicon in HNSCC. WVUCl Annual Meeting. Morgantown, WV. October 2018.

Papenberg, BW, Ingles, J, Feng, F, Allen, J, Markwell, S, Hames, R, Interval, E, Montague, A, Patel, $\mathrm{R}$, Coad, J, Wen, S, Weed, SA. Identification of a tobacco-specific molecular signature associated with disparate Appalachian HNSCC. WVUCl Annual Meeting. Morgantown, WV. October 2018. 
Papenberg, BW, Ingles, J, Feng, F, Allen, J, Markwell, S, Hames, R, Interval, E, Montague, A, Patel, $\mathrm{R}$, Coad, J, Wen, S, Weed, SA. Molecular characterization of the mortality disparity in tobaccoassociated Appalachian HNSCC. WVU Van Liere Research Day. Morgantown, WV. April 2018.

Papenberg, BW, Martinez, I, Weed, SA. Novel functions of 11q13 transcripts in invasive late stage HNSCC. WVU Van Liere Research Day. Morgantown, WV. March 2017.

Papenberg, BW, Martinez, I, Weed, SA. Evaluation of the cyclin D1 3'UTR as a competing endogenous RNA in chromosome 11q13 amplified head and neck squamous cell carcinoma. WVU Van Liere Research Day. Morgantown, WV. March 2016.

Papenberg, BW, Hayes, K, Haeseleer, F, Martinez, I, Weed, SA. Calcium-binding protein 4 is a potential driver of the $11 \mathrm{q} 13$ amplicon leading to increased cellular migration and invasion. WVU Van Liere Research Day. Morgantown, WV. February 2015.

\section{Teaching Experience}

Teaching Assistant

Microbiology Laboratory at lowa State University · June 2010 - May 2013

Student Training

Jessica Allen - Cancer Cell Biology Ph. D. Student

Eric Interval - Otolaryngology Resident

Alex Montague - Otolaryngology Resident

Robert Hickey - Undergraduate Honors Research Thesis Student

\section{Professional Skills}

Technical Skills

- Mouse Surgery for PDX Development

- Mouse Colony Management and Genotyping

- Cell Culture and Imaging

-Western Blotting and Immunofluorescence

- RNA Extraction and qRT-PCR

-Population and TCGA Datamining

Software Skills

-Adobe Creative Suite

- GraphPad Prism

- SEERStat and QGIS

-FCS Express

- Image J

. $R$ and Python

\section{Manuscripts}

Papenberg, BW, Martinez, I, Stoilov, P, Weed, SA. The CCND1 3'-UTR as a Potential Driver of Poor Outcome in Chromosome 11q13-amplified Head and Neck Squamous Cell Carcinoma. In Preparation.

Papenberg, BW, Ingles, J, Gao, Si, Feng, J, Allen, J, Markwell, S, Interval, E, Montague, A, Wen, S, Weed, SA. Copy Number Alterations Identify a Smoking-Associated Expression Signature Predictive of Poor Outcome in Head and Neck Squamous Cell Carcinoma. In Revision.

Papenberg BW, Allen JL, Markwell SM, et al. Disparate survival of late-stage male oropharyngeal cancer in Appalachia. Sci Rep. 2020;10(1):11612. 
Banday AR, Papenberg BW, Prokunina-Olsson L. When the Smoke Clears mA from a Y Chromosome-Linked IncRNA, Men Get an Increased Risk of Cancer. Cancer Res. 2020;80(13):2718-2719.

Markwell SM, Ammer AG, Interval ET, Allen JA, Papenberg BW, Hames, RA, Castaño JE, Schafer DA, Weed SA. Cortactin Phosphorylation by Casein Kinase 2 Regulates Actin-Related Protein 2/3 Complex Activity, Invadopodia Function, and Tumor Cell Invasion. Mol Cancer Res. 2019;17(4):987-1001.

\section{Contributions}

Dundar B, Markwell SM, Sharma NV, Olson CL, Mukherjee S, Brat DJ. Methods for in vitro modeling of glioma invasion: Choosing tools to meet the need. Glia. 2020;

\section{Citizenship}

United States of America

\section{Date Prepared}

July 21,2020

\section{References}

Dr. Scott Weed

Principal Investigator

- PO Box 9300

1833 HSS MBRCC

Morgantown, WV 26506

C. (304) 293-3016

M scweed@hsc.wvu.edu

Dr. Ivan Martinez

Committee Chair

n PO Box 9300

1838 HSS (MBRCC)

Morgantown, WV 26506

C. (304) 581-1934

M ivmartinez@hsc.wvu.edu

Dr. Peter Stoilov

Committee Member

n PO Box 9142

$3131 \mathrm{C}$ HSC North

64 Medical Center Drive

Morgantown, WV 26506

l. (304) 293-6334

M pstoilov@hsc.wvu.edu 